\title{
Total synthesis and biological evaluation of paenilamicins from the honey bee pathogen Paenibacillus larvae
}

Timur Bulatova, Sebastian Gensela, Andi Mainz ${ }^{\mathrm{a}}$, Tam Dang ${ }^{\mathrm{a}}$, Timm O. Koller ${ }^{\mathrm{b}}$, Kerstin Voigtc, Julia Ebeling $^{\mathrm{d}}$, Daniel N. Wilson ${ }^{\mathrm{b}}$, Elke Genersch ${ }^{\mathrm{d}}$, and Roderich D. Süssmuth ${ }^{\text {* }}$

\section{Supporting Information}

a. Fachgebiet Biologische Chemie, Institut für Chemie, Technische Universität Berlin, Strasse des 17. Juni 124, 10623 Berlin, Germany. *E-mail: roderich.suessmuth@tu-berlin.de

b. Institut für Biochemie und Molekularbiologie, Universität Hamburg, 20146 Hamburg, Germany

c. Jena Microbial Resource Collection (JMRC), Leibniz-Institut für Naturstoff-Forschung und Infektionsbiologie, Hans-Knöll-Institut, Adolf-Reichwein-Straße 23, 07745 Jena, Germany

d. Department of Molecular Microbiology, Institute for Bee Research, Friedrich-Engels-Str. 32, 16540 Hohen Neuendorf, Germany 


\section{Table of contents}

1. NMR spectroscopy data of natural PamB2 and synthetic analogs ................................3

2. General Experimental Procedures.........................................................................

3. Synthesis of non-proteinogenic amino acid building blocks and peptides .......................10

3.1. Preparation of protected $(2 R, 3 S, 5 S, 6 R)$-Agmatinamic acid (Aga) (49) ............................10

3.2. Preparation of protected (3S,5S,6S)-Galantinic acid (Gla) (40) ....................................15

3.3. Preparation of protected $(3 S, 5 R, 6 S)$-Galantinic acid (Gla) (14) ......................................19

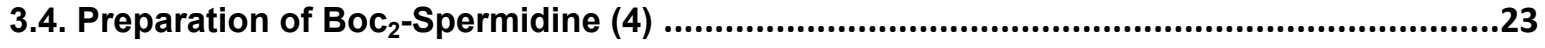

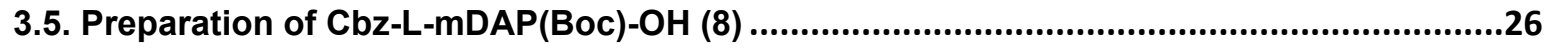

3.6. Synthesis of the protected left-hand side (LHS) fragment of PamB2 ..............................29

3.7. Synthesis of the protected right-hand side (RHS) fragment of PamB2 ….......................32

3.8. Synthesis of PamB2 - Condensation of LHS and RHS fragments and overall

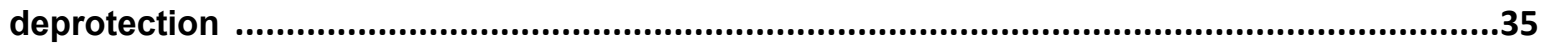

4. NMR spectra of the synthesized compounds ......................................................36

5. Antimicrobial testing experimental procedures ........................................................63

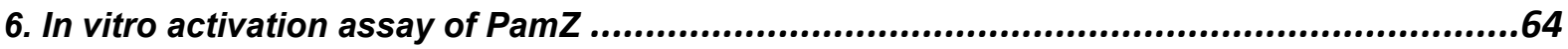

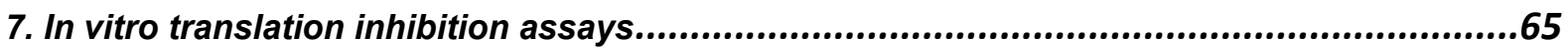

8. In vivo function experimental procedures ...........................................................67

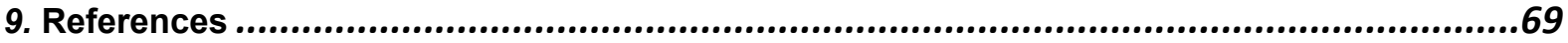




\section{NMR spectroscopy data of natural PamB2 and synthetic analogs}

Table S1. ${ }^{1} \mathrm{H}$ and ${ }^{13} \mathrm{C}$ chemical shifts (in p.p.m.) of natural and synthetic PamB2. CSPs (in p.p.m.) are calculated with respect to natural PamB2 (see Methods section). Significant CSPs (> s.d.) are highlighted in red.

\begin{tabular}{|c|c|c|c|c|c|c|c|c|c|c|c|c|}
\hline \multirow[b]{2}{*}{ Res. } & \multirow[b]{2}{*}{ Pos } & \multicolumn{2}{|c|}{ natural PamB2 } & \multicolumn{3}{|c|}{ Synth. PamB2_1 } & \multicolumn{3}{|c|}{ Synth. PamB2_2 } & \multicolumn{3}{|c|}{ Synth. PamB2_3 } \\
\hline & & $\delta^{13} \mathrm{C}$ & $\delta^{1} \mathrm{H}$ & $\delta^{13} \mathrm{C}$ & $\delta^{1} \mathrm{H}$ & CSP & $\delta^{13} \mathrm{C}$ & $\delta^{1} \mathrm{H}$ & CSP & $\delta^{13} \mathrm{C}$ & $\delta^{1} \mathrm{H}$ & CSP \\
\hline \multirow[t]{11}{*}{ Aga } & 2 & 77.34 & 4.14 & 77.34 & 4.13 & 0.01 & 77.33 & 4.13 & 0.01 & 77.34 & 4.14 & 0.00 \\
\hline & 3 & 71.09 & 4.20 & 71.06 & 4.22 & 0.02 & 71.07 & 4.22 & 0.01 & 71.14 & 4.19 & 0.02 \\
\hline & $4^{4}$ & \multirow{2}{*}{37.81} & 1.79 & \multirow{2}{*}{39.42} & 1.90 & 0.14 & \multirow{2}{*}{39.41} & 1.90 & 0.14 & \multirow{2}{*}{37.85} & 1.80 & 0.01 \\
\hline & 4“" & & 1.60 & & 1.62 & 0.10 & & 1.62 & 0.10 & & 1.61 & 0.01 \\
\hline & 5 & 69.94 & 4.12 & 69.56 & 3.92 & 0.20 & 69.57 & 3.92 & 0.20 & 69.89 & 4.12 & 0.00 \\
\hline & 6 & 58.83 & 3.37 & 58.91 & 3.23 & 0.13 & 58.92 & 3.23 & 0.13 & 58.76 & 3.37 & 0.00 \\
\hline & 7 & \multirow{2}{*}{26.76} & 1.74 & \multirow[t]{2}{*}{29.25} & 1.82 & 0.17 & \multirow[t]{2}{*}{29.24} & 1.82 & 0.17 & \multirow[t]{2}{*}{26.82} & 1.75 & 0.01 \\
\hline & $7 “$ & & 1.67 & & 1.67 & 0.15 & & 1.67 & 0.15 & & 1.67 & 0.01 \\
\hline & $8^{\prime}$ & \multirow{2}{*}{27.22} & 1.75 & \multirow{2}{*}{26.81} & 1.72 & 0.04 & \multirow{2}{*}{26.79} & 1.71 & 0.05 & \multirow{2}{*}{27.31} & 1.76 & 0.01 \\
\hline & 8“" & & 1.67 & & 1.72 & 0.05 & & 1.71 & 0.05 & & 1.67 & 0.01 \\
\hline & 9 & 43.50 & 3.24 & 43.47 & 3.24 & 0.00 & 43.47 & 3.24 & 0.00 & 43.50 & 3.24 & 0.00 \\
\hline \multirow[t]{2}{*}{ Ala } & $\alpha$ & 52.44 & 4.43 & 52.48 & 4.42 & 0.01 & 52.42 & 4.41 & 0.01 & 52.48 & 4.42 & 0.01 \\
\hline & $\beta$ & 19.10 & 1.45 & 19.15 & 1.45 & 0.00 & 19.23 & 1.45 & 0.01 & 19.18 & 1.45 & 0.01 \\
\hline \multirow[t]{4}{*}{ mDap1 } & $\alpha$ & 52.77 & 4.80 & 52.77 & 4.81 & 0.01 & 52.77 & 4.81 & 0.01 & 52.79 & 4.80 & 0.00 \\
\hline & $\beta^{\prime}$ & 5180 & 3.55 & 5169 & 3.57 & 0.02 & 5163 & 3.56 & 0.01 & 5167 & 3.56 & 0.02 \\
\hline & $\beta^{\prime \prime}$ & & 3.35 & & 3.36 & 0.01 & & 3.36 & 0.02 & & 3.36 & 0.01 \\
\hline & $\delta$ & 36.33 & 2.77 & 36.29 & 2.78 & 0.01 & 36.31 & 2.78 & 0.00 & 36.31 & 2.78 & 0.00 \\
\hline Orn & $\alpha$ & 56.58 & 4.39 & 56.55 & 4.37 & 0.02 & 56.57 & 4.39 & 0.00 & 56.59 & 4.39 & 0.00 \\
\hline & $\beta^{\prime}$ & 30.88 & 1.92 & 30.94 & 1.92 & 0.00 & 31.08 & 1.92 & 0.01 & 31.03 & 1.92 & 0.01 \\
\hline & $\beta^{\prime \prime}$ & & 1.84 & & 1.84 & 0.00 & & 1.83 & 0.02 & & 1.84 & 0.01 \\
\hline & $\gamma$ & 26.15 & 1.72 & 26.13 & 1.72 & 0.01 & 26.15 & 1.72 & 0.00 & 26.18 & 1.73 & 0.01 \\
\hline & $\delta$ & 41.79 & 3.01 & 41.67 & 3.01 & 0.01 & 41.66 & 3.01 & 0.01 & 41.67 & 3.01 & 0.01 \\
\hline Gla & $2^{\prime}$ & 46.41 & 2.58 & 4525 & 2.65 & 0.10 & 46.46 & 2.58 & 0.00 & 46.46 & 2.58 & 0.00 \\
\hline & $2^{4}$ & & 2.49 & & 2.48 & 0.07 & & 2.49 & 0.00 & & 2.49 & 0.00 \\
\hline & 3 & 68.11 & 4.26 & 69.59 & 4.29 & 0.09 & 68.08 & 4.25 & 0.01 & 68.11 & 4.25 & 0.01 \\
\hline & 4 & 43.10 & 1.62 & 42.24 & 1.77 & 0.15 & 43.17 & 1.62 & 0.00 & 43.16 & 1.62 & 0.00 \\
\hline & 5 & 69.05 & 4.02 & 71.33 & 3.86 & 0.21 & 69.06 & 4.01 & 0.01 & 69.07 & 4.01 & 0.01 \\
\hline & 6 & 58.84 & 3.89 & 58.71 & 3.93 & 0.04 & 58.86 & 3.89 & 0.00 & 58.86 & 3.89 & 0.00 \\
\hline & 7 & 63.87 & 3.73 & 62.73 & 3.79 & 0.09 & 63.91 & 3.73 & 0.01 & 63.91 & 3.73 & 0.01 \\
\hline & 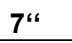 & & 3.62 & & 3.67 & 0.09 & & 3.62 & 0.00 & & 3.62 & 0.00 \\
\hline mDap2 & $\alpha$ & 52.52 & 4.88 & 52.55 & 4.87 & 0.01 & 52.55 & 4.87 & 0.01 & 52.55 & 4.87 & 0.01 \\
\hline & $\beta^{\prime}$ & 51.94 & 3.57 & 51.95 & 3.57 & 0.00 & 51.94 & 3.56 & 0.01 & 51.94 & 3.56 & 0.00 \\
\hline & $\beta^{\prime \prime}$ & & 3.34 & & 3.35 & 0.01 & & 3.35 & 0.01 & & 3.35 & 0.01 \\
\hline & $\delta$ & 36.33 & 2.77 & 36.29 & 2.78 & 0.01 & 36.31 & 2.78 & 0.00 & 36.31 & 2.78 & 0.00 \\
\hline Gly & $\alpha$ & 45.33 & 3.91 & 45.33 & 3.91 & 0.00 & 45.33 & 3.91 & 0.00 & 45.34 & 3.91 & 0.00 \\
\hline Spd & 2 & 39.49 & 3.09 & 39.38 & 3.09 & 0.01 & 39.38 & 3.09 & 0.01 & 39.38 & 3.09 & 0.01 \\
\hline & 3 & 26.60 & 2.07 & 26.56 & 2.08 & 0.01 & 26.57 & 2.08 & 0.01 & 26.56 & 2.08 & 0.01 \\
\hline & 4 & 47.29 & 3.13 & 47.28 & 3.14 & 0.01 & 47.29 & 3.13 & 0.00 & 47.28 & 3.13 & 0.00 \\
\hline & 6 & 50.19 & 3.07 & 50.18 & 3.07 & 0.01 & 50.19 & 3.07 & 0.00 & 50.19 & 3.07 & 0.00 \\
\hline & 7 & 25.69 & 1.68 & 25.79 & 1.69 & 0.01 & 25.79 & 1.69 & 0.01 & 25.77 & 1.69 & 0.01 \\
\hline & 8 & 28.19 & 1.57 & 28.31 & 1.58 & 0.01 & 28.31 & 1.57 & 0.01 & 28.31 & 1.57 & 0.01 \\
\hline & 9 & 41.44 & 3.23 & 41.46 & 3.23 & 0.00 & 41.45 & 3.23 & 0.00 & 41.45 & 3.23 & 0.00 \\
\hline
\end{tabular}




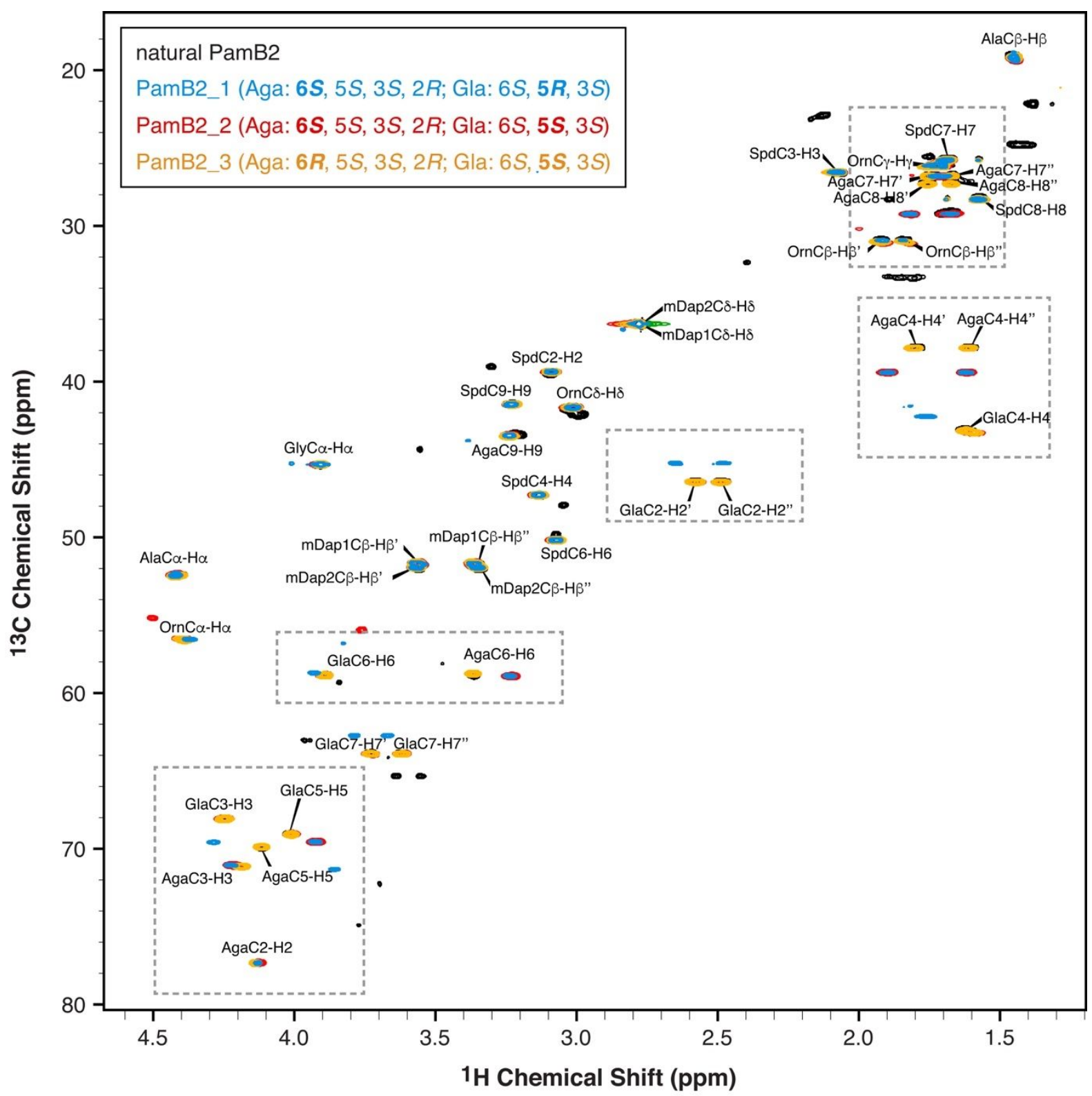

Figure S1. Overlay of ${ }^{1} \mathrm{H}-{ }^{13} \mathrm{C}$ HSQC spectra of natural and synthetic PamB2. The overlay includes the reference spectrum of natural PamB2 (black contours), synthetic PamB2_1 with the originally published 1 configuration (blue contours), as well as its diastereomers PamB2_2 with (5S)-configuration in Gla (red contours) and PamB2_3 with additionally $(6 R)$-configured Aga (orange contours). Resonance assignments refer to natural PamB2. Diagnostic regions are highlighted in dashed boxes, which are enlarged in Figure S2. 
natural PamB2

PamB2_1 (Aga: 6S, 5S, 3S, 2R; Gla: 6S, 5R, 3S)

PamB2_2 (Aga: 6S, 5S, 3S, 2R; Gla: 6S, 5S, 3S)

PamB2_3 (Aga: 6R, 5S, 3S, 2R; Gla: 6S, 5S, 3S)
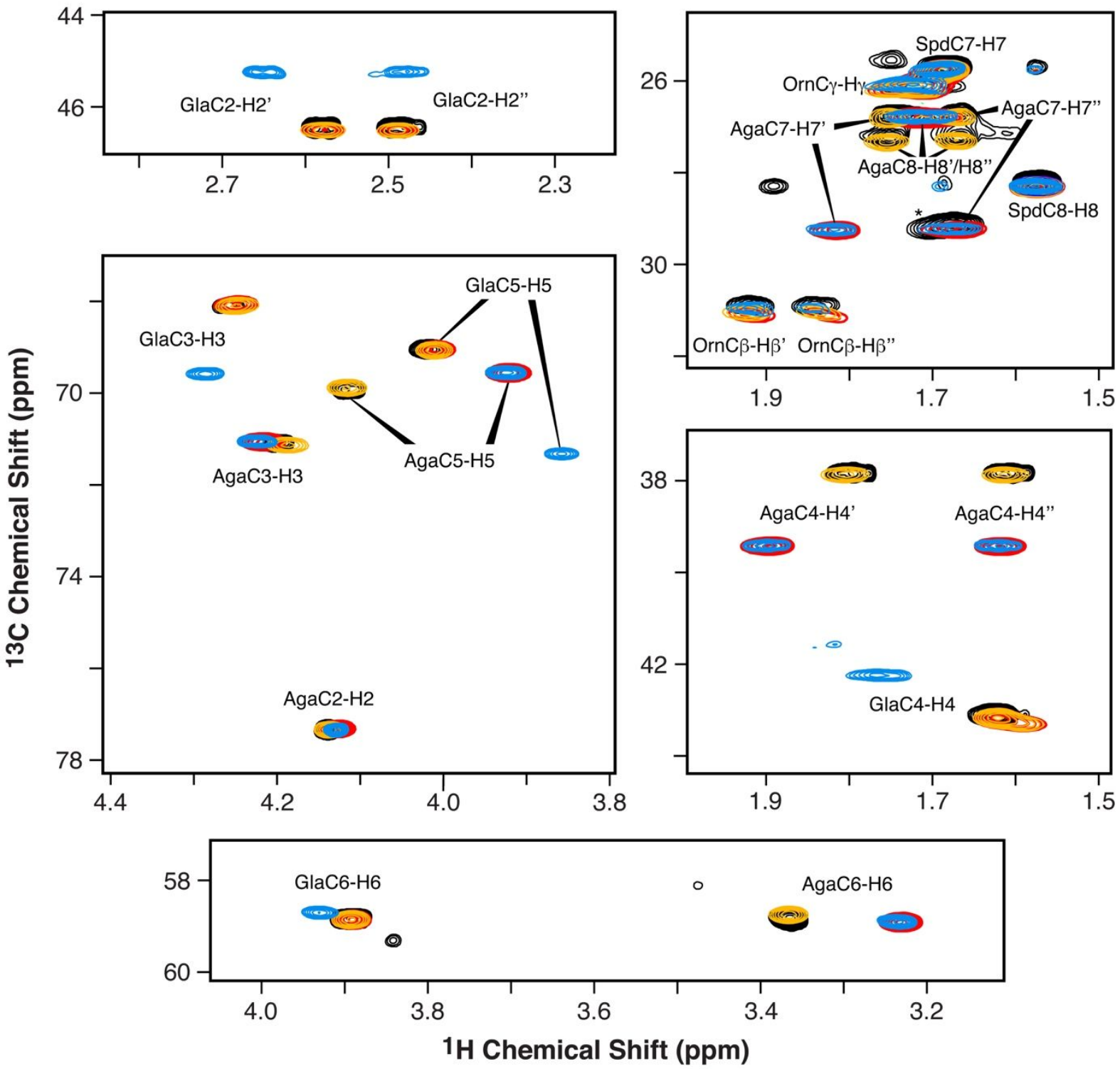

Figure S2. Diagnostic sections of ${ }^{1} \mathrm{H}-{ }^{13} \mathrm{C}$ HSQC spectra of natural and synthetic PamB2. The overlay includes the reference spectrum of natural PamB2 (black contours), synthetic PamB2_1 with the originally published configuration $^{1}$ (blue contours), as well as its diastereomers PamB2_2 with (5S)-configuration in Gla (red contours) and PamB2_3 with additionally (6R)-configured Aga (orange contours). Resonance assignments are indicated for all diastereomers. The asterisk denotes the $\mathrm{C} \delta-\mathrm{H} \delta$ correlation peak of $D$-Lys, which arises from a small contamination of PamB1 in the natural PamB2 sample. 
A

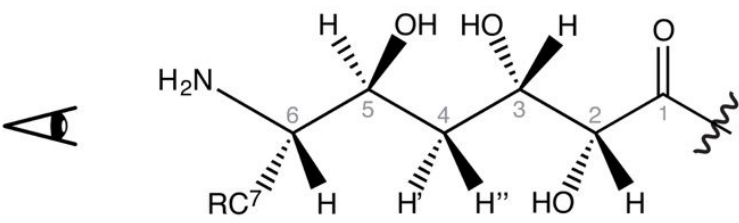

$6 S 5 S$

$3 S \quad 2 R$

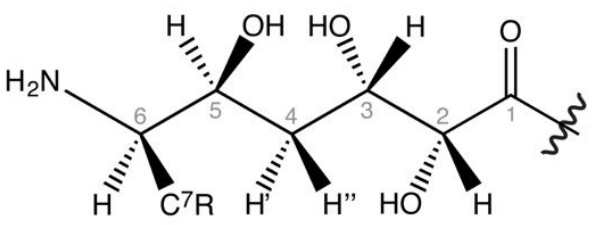

$6 R \quad 5 S$

$3 S \quad 2 R$

B

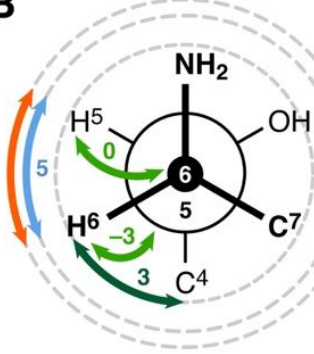
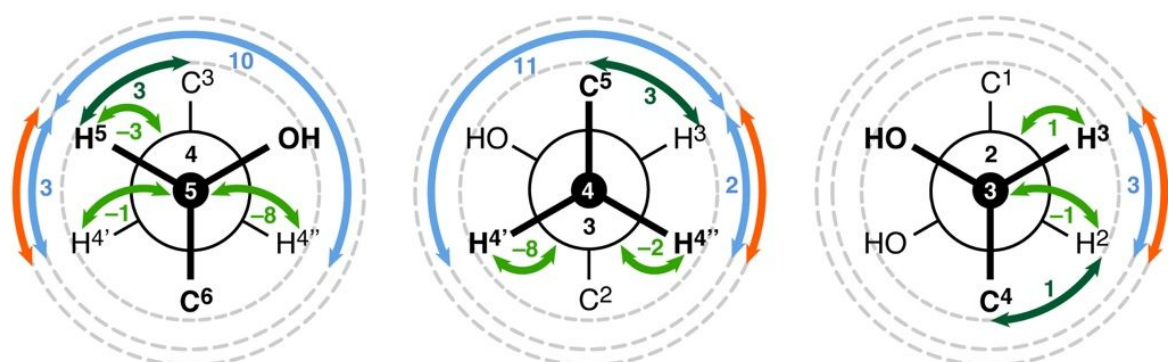

C

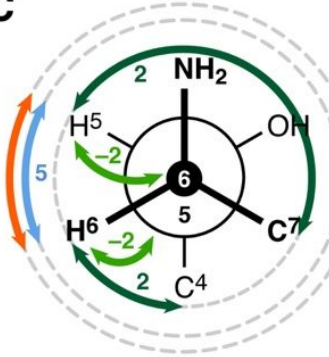

$6 R$

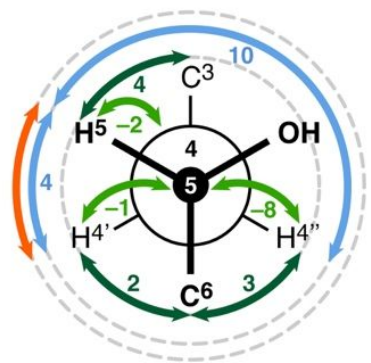

$5 S$
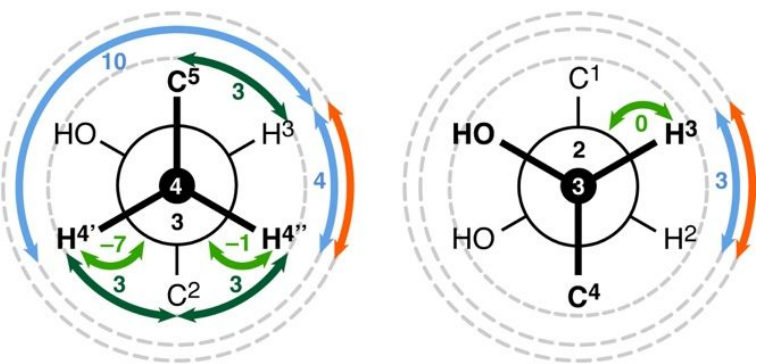

$3 S \quad 2 R$

Figure S3. Summary of NMR data on the configuration of Aga in natural and synthetic PamB2. (A) Overview of the originally published ${ }^{1}$ (left) and the here-revised configuration of Aga (right). The corresponding Newman projections of relevant $\mathrm{C}-\mathrm{C}$ bonds are illustrated for natural PamB2 (B) and synthetic PamB2_3 (C). Experimentally determined scalar coupling constants (in Hz) ${ }^{3} J_{\mathrm{HH}}$ (blue), ${ }^{2} J_{\mathrm{CH}}$ (light green) and ${ }^{3} J_{\mathrm{CH}}$ (dark green), as well as strong NOE contacts (orange) are summarized in the Newman projections. 
A

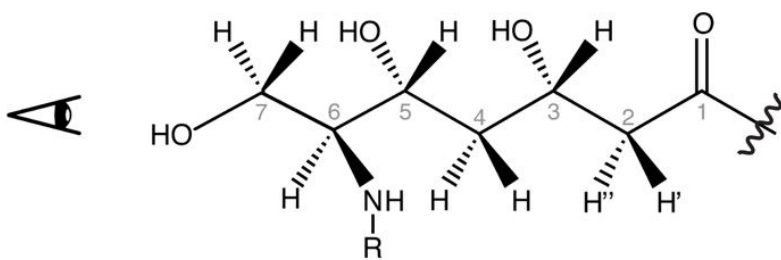

$6 S \quad 5 R$

35

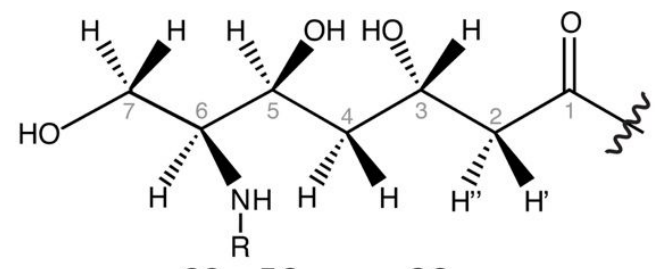

$655 S$

35
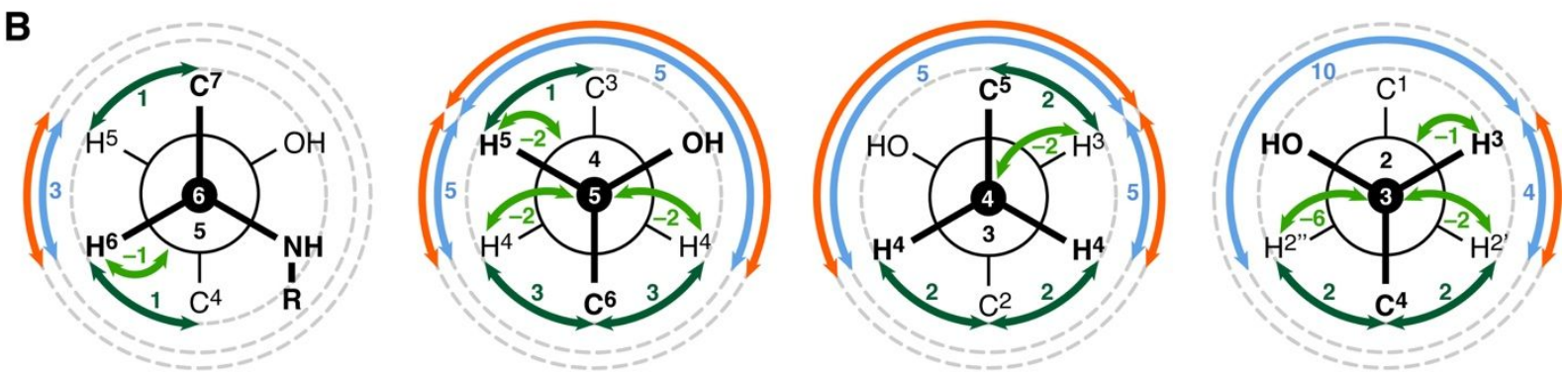

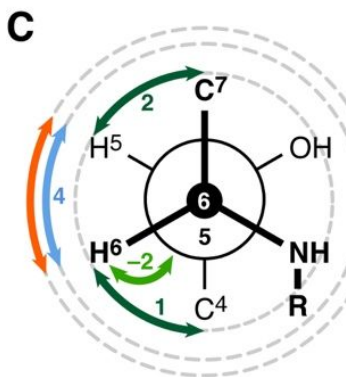

6S

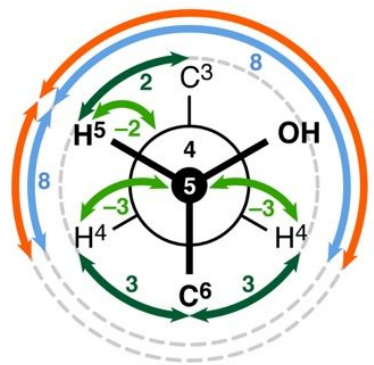

$5 S$

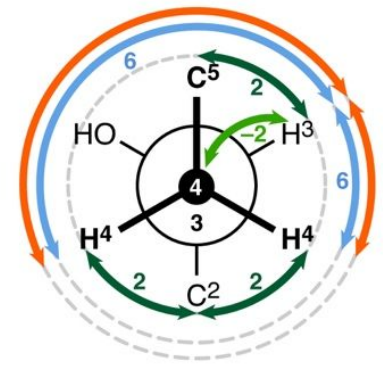

35

Figure S4. Summary of NMR data on the configuration of Gla in natural and synthetic PamB2. (A) Overview of the originally published ${ }^{1}$ (left) and the here-revised configuration of Gla (right). The corresponding Newman projections of relevant $\mathrm{C}-\mathrm{C}$ bonds are illustrated for natural PamB2 (B) and synthetic PamB2_3 (C). Experimentally determined scalar coupling constants (in $\mathrm{Hz}{ }^{3} J_{\mathrm{HH}}$ (blue), ${ }^{2} J_{\mathrm{CH}}$ (light green) and ${ }^{3} J_{\mathrm{CH}}$ (dark green), as well as strong NOE contacts (orange) are summarized in the Newman projections. Notably, the diastereotopic methylene protons $\mathrm{H}^{4}$ are isochronous, and thus scalar and dipolar couplings to neighboring nuclei cannot be differentiated. 


\section{General experimental procedures}

Commercially available reagents (Fisher Scientific International, Inc., Schwerte, Germany; Carl Roth GmbH and Co. KG, Karlsruhe, Germany; Fluorochem Ltd, Hadfield, UK; Sigma-Aldrich Taufkirchen, Germany; Iris Biotech $\mathrm{GmbH}$, Marktredwitz, Germany; Orpegen, Heidelberg, Germany; ABCR, Karlsruhe, Germany; Alfa Aesar, Karlsruhe, Germany; Merk, Darmstadt; Germany and Acros, Geel, Belgien) and solvents (Fisher Scientific-Acros, Schwerte, Germany) were used without further purification.

Analytical thin layer chromatography was carried out using silica pre-coated aluminum plates from Marcherey-Nagel, Düren, Germany (Alugram $®$ SIL G/UV254, silica gel 60). Analysis was performed by visualizing under UV light $(\lambda=254 \mathrm{~nm})$ and by staining with $\mathrm{KMnO}_{4}$ solution $\left(3 \mathrm{~g} \mathrm{KMnO}_{4}, 20 \mathrm{~g} \mathrm{~K}_{2} \mathrm{CO}_{3}\right.$, $300 \mathrm{ml}$ dist. water, $5 \mathrm{ml} \mathrm{NaOH}$ solution $(5 \%)$ ) or a ninhydrin solution $(0.3 \mathrm{~g}$ ninhydrin, $2.5 \mathrm{~mL}$ glacial acetic acid, $100 \mathrm{~mL} n-\mathrm{BuOH}$ ) and subsequent heating.

Flash chromatography was carried out was conducted using silica gel 40-63 $\mu \mathrm{m}$ from VWR Chemicals, Darmstadt, Germany and solvent systems from hexanes (Hex), ethyl acetate (EtOAc), dichloromethane (DCM), chloroform $\left(\mathrm{CHCl}_{3}\right)$ and methanol $(\mathrm{MeOH})$.

Preparative HPLC was performed with a 1260 Infinity from Agilent Technologies using Sunfire Prep C18 OBD $10 \mu \mathrm{m}, 50 \times 150 \mathrm{~mm}$ column (particle size $10 \mu \mathrm{m} \times 100 \AA$ ) from Agilent Technologies (solvent $A: \mathrm{H}_{2} \mathrm{O}+0.1 \%$ TFA, solvent $\mathrm{B}: \mathrm{MeCN}+0.1 \% \mathrm{TFA}$, flow rate $50 \mathrm{~mL} / \mathrm{min}$ ).

${ }^{1} \mathrm{H}$ and ${ }^{13} \mathrm{C}$ NMR spectra were recorded at $298 \mathrm{~K}$ using Bruker Avance-II $400 \mathrm{MHz}$, Bruker Avance-III $500 \mathrm{MHz}$ or Bruker Avance-III $700 \mathrm{MHz}$ instruments (Bruker, Karlsruhe, Germany). TopSpin 3.5 (Bruker, Karlsruhe, Germany) was used for data acquisition and processing. Spectra analysis was performed using NMRFAM-SPARKY,2,3 The chemical shifts of synthetic intermediates are reported in parts per million (ppm) using the residual solvent peak as an internal reference (DMSO- $\mathrm{d}_{6}, \mathrm{CDCl}_{3}$ or $\mathrm{D}_{2} \mathrm{O}$ ). Multiplicity ( $\mathrm{br} \mathrm{s}=$ broad singlet, $\mathrm{s}=$ singlet, $\mathrm{d}=$ doublet, $\mathrm{dd}=$ doublet of doublet, $\mathrm{t}=$ triplet, $\mathrm{q}=$ quartet, $\mathrm{m}=$ multiplet) and coupling constants $(\mathrm{J}$ in $\mathrm{Hz})$ are quoted where possible. NMR spectra of natural and synthetic PamB2 were recorded using samples in $\mathrm{D}_{2} \mathrm{O}$ with $0.1 \%$ acetic acid- $\mathrm{d}_{4}$ at $298 \mathrm{~K}$. Finger-print ${ }^{1} \mathrm{H}-{ }^{13} \mathrm{C} \mathrm{HSQC}$ spectra were recorded with acquisition times of $120 \mathrm{~ms}$ and $9 \mathrm{~ms}$ in the direct ${ }^{1} \mathrm{H}$ and indirect ${ }^{13} \mathrm{C}$ dimension, respectively. A delay $\Delta / 2$ of $1.72 \mathrm{~ms}$ was used for INEPT transfers corresponding to ${ }^{1} \mathrm{~J}_{\mathrm{HC}}$ of $145 \mathrm{~Hz}$. A $90^{\circ}$-shifted squared sine-bell function was used for apodization of time domain data. The $2 \mathrm{D}$ data was processed by applying linear forward prediction in the indirect ${ }^{13} \mathrm{C}$ dimension and zero filling prior to Fourier transformation. ${ }^{1} \mathrm{H}$ chemical shifts were referenced externally using a sample of trimethylsilylpropanoic acid (TMSP- $d_{4}$, Deutero $\mathrm{GmbH}$, Kastellaun, Germany) in $\mathrm{D}_{2} \mathrm{O}$ with $0.1 \%$ acetic acid-d4 measured at $298 \mathrm{~K} .{ }^{13} \mathrm{C}$ chemical shifts were referenced indirectly using a correction factor of $f_{13 \mathrm{C} / 1 \mathrm{H}}=0.251449530 .{ }^{4,5}$ Chemical shift perturbations (CSPs) were calculated using the following equation: ${ }^{6}$

$$
C S P=\sqrt{(f \times \delta 13 \mathrm{C})^{2}+(\delta 1 \mathrm{H})^{2}}
$$


where $\Delta \delta_{13 \mathrm{C}}$ and $\Delta \delta_{1 \mathrm{H}}$ correspond to the ${ }^{13} \mathrm{C}$ and ${ }^{1} \mathrm{H}$ chemical shift differences between natural PamB2 and the corresponding synthetic PamB2 for each carbon-proton pair. We used a weighting factor $f$ of 0.06 to account for the much larger chemical shift dispersion in the ${ }^{13} \mathrm{C}$ dimension (ca. $60 \mathrm{ppm}$ ) compared to that in the ${ }^{1} \mathrm{H}$ dimension (ca. $3.5 \mathrm{ppm}$ ). Scalar coupling constants were extracted from ${ }^{1} \mathrm{H}$ 1D, ${ }^{1} \mathrm{H}-{ }^{1} \mathrm{H}$ COSY-DQF and ${ }^{1} \mathrm{H}-{ }^{13} \mathrm{C}$ HSQC-HECADE spectra. ${ }^{7}$ For the latter, homonuclear DIPSI2 mixing with a duration of $60 \mathrm{~ms}$ was employed to determine long-range ${ }^{3,4} \mathrm{~J}_{\mathrm{HC}}$ couplings. ROESY experiments were conducted using a 300-ms spin lock with a radio-frequency field strength of $3 \mathrm{kHz}$ composed of two inversion pulses of alternating phase in order to suppress TOCSY artefacts. ${ }^{8}$

HPLC-HRMS spectra were recorded on a QTrap LTQ XL (Thermo Fisher Scientific, Waltham, Massachusetts, USA) with an Agilent 1200 Series HPLC-System (Agilent Technologies, Waldbronn, Germany) with a C18 column (50 x $2 \mathrm{~mm}$, particle size $3 \mu \mathrm{m})$.

HPLC-ESI-MS coupled measurements were performed on an Orbitrap XL-mass spectrometer from Thermo Scientific (Waltham, MA, USA) coupled with 1200-HPLC from Agilent Technologies using a hypersil 100-C18-column from Thermo Scientific(solvent A: $\mathrm{H}_{2} \mathrm{O}+0.1 \% \mathrm{HCOOH}$, solvent B: MeCN+ $0.1 \% \mathrm{HCOOH}$; flow rate $1.3 \mathrm{~mL} / \mathrm{min}$ ). Xcalibur (Thermo Scientific) was used for the evaluation of the spectra.

Analytical HPLC was performed with a 1100 Infinity from Agilent Technologies using an Agilent Eclipse XDB column $(4.0 \times 50 \mathrm{~mm}, 5 \mu \mathrm{m})$ from Agilent Technologies (solvent $\mathrm{A}: \mathrm{H}_{2} \mathrm{O}+0.1 \% \mathrm{HCOOH}$, solvent B: $\mathrm{MeCN}+0.1 \% \mathrm{HCOOH}$, flow rate $2 \mathrm{~mL} / \mathrm{min}$ ). 


\section{Synthesis of non-proteinogenic amino acid building blocks and peptides}

\subsection{Preparation of protected $(2 R, 3 S, 5 S, 6 R)$-Agmatinamic acid (Aga) (49)}

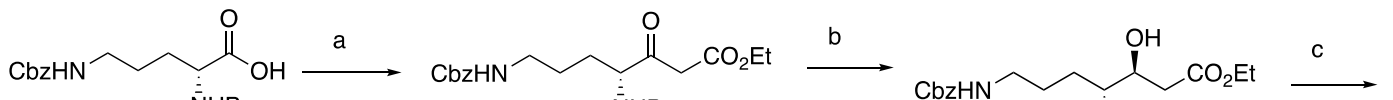

41 N̄HBoc

42 NHBoc

$43 \quad \overline{\bar{N}} \mathrm{HBOC}$
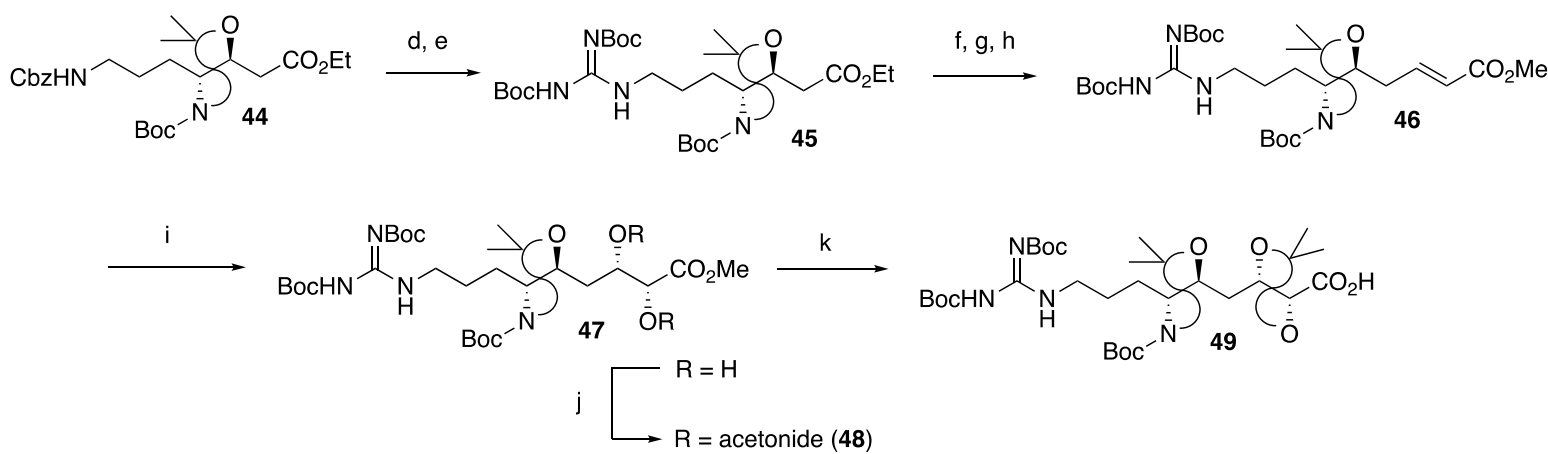

Scheme S1: Synthesis of $(2 R, 3 S, 5 S, 6 R)$-Agmatinamic acid (Aga):

a) Monoethyl malonate potassium salt, carbonyldiimidazole, $\mathrm{Et}_{3} \mathrm{~N}, \mathrm{MgCl}_{2} \mathrm{THF}, 0{ }^{\circ} \mathrm{C}$ to rt, $24 \mathrm{~h}, 75 \%$; b) $\mathrm{KBH}_{4}$, $\mathrm{MeOH},-40{ }^{\circ} \mathrm{C}, 4 \mathrm{~h}, 51 \%$; c) 2,2-dmp, pTsOH, DCM, rt, 4 h, 98\%; d) $\mathrm{H}_{2} / \mathrm{Pd} / \mathrm{C}$, EtOAc, rt, 2 h; e) pyrrazole 20 , DIPEA, EtOH, rt, $12 \mathrm{~h}$, (82\% over 2 steps); f) DIBALH, DCM, THF, $\left.0{ }^{\circ} \mathrm{C}, 1.5 \mathrm{~h} ; \mathrm{g}\right) \mathrm{SO}_{3} \cdot$ Pyr, DMSO, DIPEA, DCM, $0{ }^{\circ} \mathrm{C}, 1 \mathrm{~h}$; h) $\mathrm{Ph}_{3} \mathrm{PCHCO}_{2} \mathrm{Me}, \mathrm{DCM}, \mathrm{rt}, 18 \mathrm{~h}$, (64\% over 3 steps); i) AD-Mix alpha, $\mathrm{MeSO}_{2} \mathrm{NH}_{2}, \mathrm{THF} \mathrm{H}_{2} \mathrm{O} 1: 1,0{ }^{\circ} \mathrm{C}$ to rt, 24 h, 61\%; j) 2,2-dmp, pTsOH, DCM, reflux, 24 h, 78\%; k) LiOH, $\mathrm{H}_{2} \mathrm{O}, \mathrm{MeOH}, \mathrm{THF}, \mathrm{rt}, 2 \mathrm{~h}, 91 \%$.

(4R)-N,N -Boc-Cbz-ethyl-4,8-diamino-3-oxooctanoate (42)<smiles>CCOC(=O)CC(=O)[C@H](CCCNC(=O)OCc1ccccc1)NC(=O)O</smiles>

A solution of N-Cbz, N-Boc protected D-ornithine $(5.27 \mathrm{~g}, 14.4 \mathrm{mmol}, 1$ eq.) in $25 \mathrm{ml} \mathrm{THF}$ was cooled to $0^{\circ} \mathrm{C}$ and $1,1^{\prime}$-carbonyldiimidazole (CDI) $(2.57 \mathrm{~g}, 15.84 \mathrm{mmol}, 1.1$ eq. $)$ was added in small portions under vigorous stirring. After evolution of gas the mixture was stirred for $3 \mathrm{~h}$ at room temperature and then cooled in an ice bath. To a suspention of monoethyl malonate potassium salt $(4.9 \mathrm{~g}, 28.8 \mathrm{mmol}, 2$ eq.) in $50 \mathrm{ml} \mathrm{THF}$ at $5{ }^{\circ} \mathrm{C}$ was added $\mathrm{Et}_{3} \mathrm{~N}\left(6.38 \mathrm{ml}, 46.13 \mathrm{mmol}, 3.2\right.$ eq.) followed by anhydrous $\mathrm{MgCl}_{2}$ ( $3.83 \mathrm{~g}, 40.31 \mathrm{mmol}, 2.8$ eq.). The mixture was stirred at room temperature for additional 3 hours then cooled to $0{ }^{\circ} \mathrm{C}$ and the solution of the activated ester was added dropwise over $30 \mathrm{~min}$. The resulting mixture was left to stir for 24 hours at room temperature. The mixture was quenched with aqueous citric acid, the aqueous phase was separated and extracted with EtOAc three times. The combined organic layers were washed with saturated $\mathrm{NaHCO}_{3}$ and brine, dried over $\mathrm{Na}_{2} \mathrm{SO}_{4}$, filtered and the solvent was removed in vacuo. Purification with column chromatography $\left(\mathrm{SiO}_{2}\right.$, Hex:EtOAc - 1:1) led to the ester 42 $(4.71 \mathrm{~g}, 75 \%)$ as a white solid. ${ }^{1} \mathrm{H}-\mathrm{NMR}\left(400 \mathrm{MHz}, \mathrm{CDCl}_{3}\right): \delta(\mathrm{ppm})=1.26(\mathrm{t}, \mathrm{J}=7.1 \mathrm{~Hz}, 3 \mathrm{H}), 1.43(\mathrm{~s}$, $9 \mathrm{H}), 1.55(\mathrm{~m}, 3 \mathrm{H}), 1.91(\mathrm{~m}, 1 \mathrm{H}), 3.21-3.23(\mathrm{~m}, 2 \mathrm{H}), 3.48-3.57(\mathrm{~m}, 2 \mathrm{H}), 4.18(\mathrm{q}, \mathrm{J}=7.1 \mathrm{~Hz}, 2 \mathrm{H}), 4.37$ $(\mathrm{m}, 1 \mathrm{H}), 4.93(\mathrm{~m}, 1 \mathrm{H}), 5.08(\mathrm{~s}, 2 \mathrm{H}), 5.18(\mathrm{~m}, 1 \mathrm{H}), 7.28-7.35(\mathrm{~m}, 5 \mathrm{H}) .{ }^{13} \mathrm{C}-\mathrm{NMR}\left(100 \mathrm{MHz}, \mathrm{CDCl}_{3}\right)$ : 
$\delta(\mathrm{ppm})=14.2,26.0,28.2,28.4,40.5,59.3,61.7,66.8,80.4,128.2,128.3,128.7,136.7,155.6,156.6$ 167.1, 202.2. HRMS (ESI) $\mathrm{m} / \mathrm{z}$ calc for $\mathrm{C}_{22} \mathrm{H}_{32} \mathrm{~N}_{2} \mathrm{O}_{7}[\mathrm{M}+\mathrm{H}]^{+} 436.2210$ found 436.2237 .

(4R)-N,N -Boc-Cbz-ethyl-4,8-diamino-(3S)-hydroxyoctanoate (43)<smiles>CCOC(=O)C[C@@H](O)[C@H](CCCNC(=O)OCc1ccccc1)NC(=O)OCc1ccccc1</smiles>

$\mathrm{KBH}_{4}(2.0 \mathrm{~g}, 37 \mathrm{mmol}, 3.5 \mathrm{eq}$.) was added in small portions to a stirred solution of $42(4.7 \mathrm{~g}, 11 \mathrm{mmol}$, 1 eq.) in $\mathrm{MeOH}(150 \mathrm{~mL})$ at $-40^{\circ} \mathrm{C}$. After $5 \mathrm{~h}$ at this temperature, the reaction was quenched with $10 \%$ aqueous citric acid at $0{ }^{\circ} \mathrm{C}$ (adjusted to $\mathrm{pH} 3$ ). After concentration of the reaction mixture in vacuo, water (30 mL) was added, and the resulting mixture was extracted with $\mathrm{CH}_{2} \mathrm{Cl}_{2}(4 \times 30 \mathrm{~mL})$. The combined extracts were washed with brine $(2 \times 30 \mathrm{~mL})$ and then dried over $\mathrm{Na}_{2} \mathrm{SO}_{4}$. Concentration of the solvent in vacuo afforded a mixture of $(3 S, 4 R)$ and $(3 R, 4 R)$ diastereomers $(3: 1)$ of 43 , which was separated by preparative reversed phase (C18) HPLC (isocratic elution, $\mathrm{MeCN} / \mathrm{H}_{2} \mathrm{O}-1: 1,50 \mathrm{ml} / \mathrm{min}$ ) to give an individual $(3 S, 4 R)$-diastereomer as a white solid $(2.46 \mathrm{~g}, 51 \%) .{ }^{1} \mathrm{H}-\mathrm{NMR}\left(500 \mathrm{MHz}, \mathrm{CDCl}_{3}\right): \delta(\mathrm{ppm})=$ 1.26 (t, J = 7.1 Hz, $3 \mathrm{H}), 1.37(\mathrm{~m}, 1 \mathrm{H}), 1.43(\mathrm{~s}, 9 \mathrm{H}), 1.51(\mathrm{~m}, 1 \mathrm{H}), 1.62-1.65(\mathrm{~m}, 2 \mathrm{H})$, 2.44-2.48 (m, 2 $\mathrm{H}), 3.21(\mathrm{~m}, 2 \mathrm{H}), 3.57(\mathrm{~m}, 1 \mathrm{H}), 3.95-3.97(\mathrm{~m}, 1 \mathrm{H}), 4.16(\mathrm{q}, \mathrm{J}=7.1 \mathrm{~Hz}, 2 \mathrm{H}), 4.73-4.75(\mathrm{~m}, 1 \mathrm{H}), 4.93$ $(\mathrm{m}, 1 \mathrm{H}), 5.08(\mathrm{~s}, 2 \mathrm{H}), 7.28-7.35(\mathrm{~m}, 5 \mathrm{H}) .{ }^{13} \mathrm{C}-\mathrm{NMR}\left(126 \mathrm{MHz}, \mathrm{CDCl}_{3}\right): \delta(\mathrm{ppm})=14.3,26.7,27.1$, 28.5, 38.4, 40.9, 52.2, 61.0, 66.7, 71.0, 79.8, 128.2, 128.2, 128.6, 128.6, 136.8, 156.2, 156.6, 173.0. HRMS (ESI) $\mathrm{m} / \mathrm{z}$ calc for $\mathrm{C}_{22} \mathrm{H}_{34} \mathrm{~N}_{2} \mathrm{O}_{7}[\mathrm{M}+\mathrm{H}]^{+} 439.2444$ found 439.2414 .

(4R)-N,N -Boc-Cbz-ethyl-4,8-diamino-(3S)-oxy-N,O-acetonidooctanoate (44)<smiles>CCOC(=O)C[C@@H]1OC(C)(C)O[C@H]1CCCNC(=O)OCc1ccccc1</smiles>

Hydroxy-ester 43 (1.71 g, $3.9 \mathrm{mmol}, 1.00$ eq.) was dissolved in $17 \mathrm{~mL}$ of DCM and $4.75 \mathrm{~mL}$ 2,2dimethoxypropane (38.9 mmol, 10.0 eq.) and $p \mathrm{TsOH}^{*} \mathrm{H}_{2} \mathrm{O}(148 \mathrm{mg}, 0.78 \mathrm{mmol}, 0.2$ eq.) were added. The solution was stirred for $4 \mathrm{~h}$ at room temperature and after conversion control the reaction was stopped with a few drops $\mathrm{Et}_{3} \mathrm{~N}$. The solvent was removed under reduced pressure and the residue was purified by column chromatography $\left(\mathrm{SiO}_{2}, \mathrm{Hex} / \mathrm{EtOAc}-3.5: 1\right)$ to give the $\mathrm{N}, \mathrm{O}$-protected hydroxyl-ester $44(1.83 \mathrm{~g}, 98 \%)$ as yellowish oil. ${ }^{1} \mathrm{H}-\mathrm{NMR}\left(500 \mathrm{MHz}, \mathrm{CDCl}_{3}\right): \delta(\mathrm{ppm})=1.25(\mathrm{t}, \mathrm{J}=7.1 \mathrm{~Hz}, 3 \mathrm{H}), 1.44$ (s, $3 \mathrm{H}), 1.47$ (s, $9 \mathrm{H}), 1.51$ (s, $3 \mathrm{H}), 1.44-1.59(\mathrm{~m}, 4 \mathrm{H}), 2.53$ (dd, J = 16.5, $6.8 \mathrm{~Hz}, 1 \mathrm{H}), 2.65$ (td, J = J $=16.5,6.8 \mathrm{~Hz}, 1 \mathrm{H}), 3.16-3.30(\mathrm{~m}, 2 \mathrm{H}), 3.93$ and $4.05(\mathrm{~m}$, conform., $1 \mathrm{H}), 4.16(\mathrm{q}, \mathrm{J}=7.1 \mathrm{~Hz}, 2 \mathrm{H})$, 4.40-4.45 (m, $1 \mathrm{H}), 4.77$ and 5.31 (m, conform., $1 \mathrm{H}), 5.09(\mathrm{~s}, 2 \mathrm{H}), 7.28-7.36(\mathrm{~m}, 5 \mathrm{H}) .{ }^{13} \mathrm{C}-\mathrm{NMR}(126$ $\left.\mathrm{MHz}, \mathrm{CDCl}_{3}\right): \delta(\mathrm{ppm})=14.3,21.2,26.1,28.3,28.6,29.1,29.9,40.5,60.5,60.9,61.9,66.7,67.2,76.0$, 94.0, 128.2, 128.6, 136.7, 152.3, 156.5, 170.65. NMR spectra contain EtOAc signals. HRMS (ESI) m/z calc for $\mathrm{C}_{25} \mathrm{H}_{38} \mathrm{~N}_{2} \mathrm{O}_{7}[\mathrm{M}+\mathrm{H}]^{+} 479.2757$ found 479.2753 . 
(4R)-N,N -Boc- Arg(Boc 2 )-ethyl-4,8-diamino-(3S)-oxy-N,O-acetonidooctanoate (45)<smiles>CCOC(=O)CC1OC(C)(C)OC1CCCCNC(=N[R6](=O)OCc1ccccc1)NC(=O)OCc1ccccc1</smiles>

Protected ester 44 (1.80 g, $3.77 \mathrm{mmol}, 1.00$ eq.) was dissolved in $35 \mathrm{~mL}$ of EtOAc and $10 \% \mathrm{Pd} / \mathrm{C}$ (180 $\mathrm{mg}, 10 \mathrm{w} / \mathrm{w} . \%$ ) were added. A balloon containing $\mathrm{H}_{2}$ was attached to the reaction flask. After stirring for $2 \mathrm{~h}$ at room temperature the solution was filtered through a pad of celite with several EtOAc washings. Evaporation under reduced pressure gave the free amine $(1.28 \mathrm{~g}, 99 \%)$ as colorless oil, which was used directly in the next step.

Pyrrazole 20 (1.09 g, $3.52 \mathrm{mmol}, 1.00$ eq.) was dissolved in $15 \mathrm{~mL}$ of EtOH. In a second flask the above amine (1.28 g, $3.7 \mathrm{mmol}, 1.05$ eq.) was dissolved in $15 \mathrm{~mL}$ of EtOH and DIPEA (1.34 mL, $7.7 \mathrm{mmol}$, 2.20 eq.) was added. The solution containing the amine and DIPEA was cannulated into the pyrrazole solution and was then stirred over night at room temperature. EtOH was removed under reduced pressure and EtOAc was added. The organic phase was washed with $0.5 \mathrm{M} \mathrm{HCl}(2 \mathrm{x})$, brine, dried over $\mathrm{Na}_{2} \mathrm{SO}_{4}$, filtered and the solvent was removed under reduced pressure. Column chromatography $\left(\mathrm{SiO}_{2}\right.$, Hex:EtOAc - 5:1) gave the title compound $45(1.52 \mathrm{~g}, 82 \%)$ as a colorless solid. ${ }^{1} \mathrm{H}-\mathrm{NMR}(500 \mathrm{MHz}$, DMSO-d $\left.\mathrm{d}_{6}\right): \delta(\mathrm{ppm})=1.19(\mathrm{t}, \mathrm{J}=7.1 \mathrm{~Hz}, 3 \mathrm{H}), 1.32(\mathrm{~s}, 3 \mathrm{H}), 1.38(\mathrm{~s}, 9 \mathrm{H}), 1.39(\mathrm{~s}, 9 \mathrm{H}), 1.47(\mathrm{~s}, 9 \mathrm{H})$, $1.48(\mathrm{~s}, 3 \mathrm{H}), 1.35-1.50(\mathrm{~m}, 3 \mathrm{H}), 1.54-1.65(\mathrm{~m}, 2 \mathrm{H}), 1.85-1.94(\mathrm{~m}, 2 \mathrm{H}), 3.52-3.63(\mathrm{~m}, 1 \mathrm{H}), 3.92-3.98$ $(\mathrm{m}, 1 \mathrm{H}), 4.13$ (q, J = 7.1 Hz, $2 \mathrm{H}), 4.17-4.23(\mathrm{~m}, 1 \mathrm{H}), 8.35$ (br s, $1 \mathrm{H}), 11.52(\mathrm{~s}, 1 \mathrm{H}) .{ }^{13} \mathrm{C}-\mathrm{NMR}(126$ $\left.\mathrm{MHz}, \mathrm{DMSO}-\mathrm{d}_{6}\right): \delta(\mathrm{ppm})=13.9,24.9,25.6,27.0,27.6,27.9,28.0,60.8,61.5,75.6,77.9,78.3,82.8$, $110.1,151.1,152.1,155.2,163.1,169.9$. NMR spectra contain $\mathrm{H}_{2} \mathrm{O}$ signals.

HRMS (ESI) $\mathrm{m} / \mathrm{z}$ calc for $\mathrm{C}_{28} \mathrm{H}_{50} \mathrm{~N}_{4} \mathrm{O}_{9}[\mathrm{M}+\mathrm{H}]^{+} 587.3656$ found 587.3647 .

\section{(2E,5S,6R)-N1,N10,N11-tri-Boc-methyl-6,9-diamino-5-hydroxy-6,7-N,O- dimethyloxazolidine-non-2- enoate (46)}<smiles>COC(=O)/C=C/CC1OC(C)(C)C(CCCNC(=NC(=O)OC(C)(C)C)NC(=O)OCc2ccccc2)N1C(=O)OC(C)(C)C</smiles>

Ester 45 (1.50 g, $2.6 \mathrm{mmol}, 1.00$ eq.) was suspended in $3 \mathrm{~mL}$ of THF. DIBAL-H (1 M in DCM, $7 \mathrm{~mL}$, $7.00 \mathrm{mmol}, 2.70$ eq.) was added at $0{ }^{\circ} \mathrm{C}$ and the solution was stirred at this temperature for $30 \mathrm{~min}$. The reaction was quenched with aqueous $10 \%$ Rochelle salt solution, volatiles were removed under reduced pressure and the aqueous residue was extracted with EtOAc three times, washed with $\mathrm{H}_{2} \mathrm{O}$ and brine, dried over $\mathrm{Na}_{2} \mathrm{SO}_{4}$, filtered and concentrated under reduced pressure. The resulting alcohol was dissolved in $50 \mathrm{~mL}$ of DCM and at $0{ }^{\circ} \mathrm{C}$ DMSO (8.39 mL, $107.5 \mathrm{mmol}, 42.00$ eq.), DIPEA (7.12 mL, $41.0 \mathrm{mmol}, 16.00$ eq.) and $\mathrm{SO}_{3}{ }^{*}$ pyridine-complex ( $3.26 \mathrm{~g}, 20.4 \mathrm{mmol}, 8.00 \mathrm{eq}$.) were added. The mixture was stirred for $1 \mathrm{~h}$ at $0{ }^{\circ} \mathrm{C}$ and then treated with sat. $\mathrm{NH}_{4} \mathrm{Cl}$ solution. The phases were separated and the aqueous phase extracted with $\mathrm{DCM}$ three times. After drying over $\mathrm{Na}_{2} \mathrm{SO}_{4}$, filtration and 
concentration under reduced pressure the resulting aldehyde was introduced directly in the next step without further purification. The aldehyde was dissolved in $15 \mathrm{~mL}$ of DCM and at $0{ }^{\circ} \mathrm{CPh}_{3} \mathrm{PCHCO}_{2} \mathrm{Me}$ (1.28 g, $3.83 \mathrm{mmol}, 1.50 \mathrm{eq}$.) in $15 \mathrm{~mL}$ of DCM was added dropwise. The solution was stirred for $18 \mathrm{~h}$ at room temperature and after removing of the solvent column chromatography $\left(\mathrm{SiO}_{2}\right.$, Hex/EtOAc - 6:1) gave the title compound 46 (0.98 g, 64\% over three steps, $E / Z=5.5 / 1)$ as a yellow oil. Double bond isomers were separated at this stage. ${ }^{1} \mathrm{H}-\mathrm{NMR}\left(500 \mathrm{MHz}, \mathrm{CDCl}_{3}\right): \delta(\mathrm{ppm})=1.45(\mathrm{~s}, 9 \mathrm{H}), 1.48(\mathrm{~s}, 9 \mathrm{H})$, 1.49 (s, $9 \mathrm{H}), 1.49$ (s, $6 \mathrm{H}), 1.55-1.57$ (m, $1 \mathrm{H}), 1.60-1.67$ (m, $2 \mathrm{H}), 2.40-2.55$ (m, $2 \mathrm{H}), 3.40-3.56$ (m, 2 H), $3.73(\mathrm{~s}, 3 \mathrm{H}), 3.81-4.16(\mathrm{~m}, 2 \mathrm{H}), 5.95(\mathrm{~d}, \mathrm{~J}=15.6 \mathrm{~Hz}, 1 \mathrm{H}), 6.94(\mathrm{dt}, \mathrm{J}=15.6 \mathrm{~Hz}, \mathrm{~J}=6.7 \mathrm{~Hz}, 1 \mathrm{H})$, 8.37-8.75 (m, $1 \mathrm{H}), 11.48$ (s, $1 \mathrm{H}) .{ }^{13} \mathrm{C}-\mathrm{NMR}\left(126 \mathrm{MHz}, \mathrm{CDCl}_{3}\right): \delta(\mathrm{ppm})=25.5,28.2,28.4,28.4,28.6$, 40.8, 51.6, 61.53, 79.4, 80.3, 83.3, 123.9, 144.4, 153.5, 156.3, 163.7, 166.7. NMR spectra contain EtOAc signals. HRMS (ESI) $\mathrm{m} / \mathrm{z}$ calc for $\mathrm{C}_{29} \mathrm{H}_{50} \mathrm{~N}_{4} \mathrm{O}_{9}[\mathrm{M}+\mathrm{H}]^{+} 599.3656$ found 599.3649 .

$(2 R, 3 S, 5 S, 6 R)-N 1, N 10, N 11$-tri-Boc-methyl-6-amino-9-guanidino-2,3,5-trihydroxy-6,7$\mathrm{N}, \mathrm{O}$ - dimethyloxazolidine-nonanoate (47)

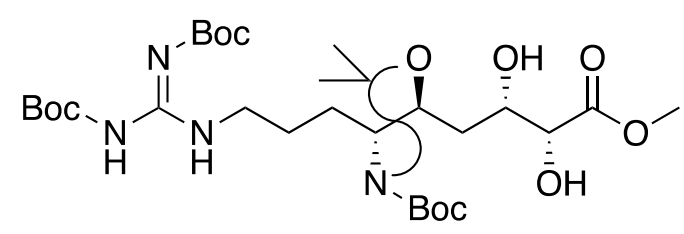

To a solution of AD-Mix-alpha (7.00 g, $4.5 \mathrm{~g} / 1 \mathrm{mmol}$ of 46 ) in $80 \mathrm{~mL}$ of $\mathrm{H}_{2} \mathrm{O}$ and $20 \mathrm{~mL}$ of THF was added methane sulfonamide ( $151.0 \mathrm{mg}, 1.61 \mathrm{mmol}, 1.00 \mathrm{eq}$.) at room temperature. After the mixture turned clear it was cooled to $0{ }^{\circ} \mathrm{C}$ and stirred for $0.5 \mathrm{~h}$. Next a solution of unsaturated ester $\mathbf{4 7}(0,96 \mathrm{~g}$, $1.61 \mathrm{mmol}, 1.00$ eq.) in $20 \mathrm{~mL}$ of THF was added. The mixture was stirred for $6 \mathrm{~h}$ at $0{ }^{\circ} \mathrm{C}$ and for $12 \mathrm{~h}$ at room temperature. The reaction was cooled to $0{ }^{\circ} \mathrm{C}$ and approx. $5 \mathrm{~g}$ of $\mathrm{Na}_{2} \mathrm{SO}_{3}$ were added. After 1 $h$ the mixture was extracted with EtOAc four times and the organics were washed with $2 \mathrm{~N} \mathrm{NaOH}$, brine, dried over $\mathrm{Na}_{2} \mathrm{SO}_{4}$, filtered and the solvent was removed under reduced pressure. Purification by column chromatography $\left(\mathrm{SiO}_{2}\right.$, Hex:EtOAc - 1:1) gave the title compound $47(608 \mathrm{mg}, 61 \%)$ as a colorless solid. ${ }^{1} \mathrm{H}-\mathrm{NMR}\left(500 \mathrm{MHz}, \mathrm{CDCl}_{3}\right): \delta(\mathrm{ppm})=1.44(\mathrm{~s}, 9 \mathrm{H}), 1.47(\mathrm{~s}, 9 \mathrm{H}), 1.48(\mathrm{~s}, 9 \mathrm{H}), 1.48(\mathrm{~s}$, $6 \mathrm{H}), 1.58-1.68(\mathrm{~m}, 3 \mathrm{H}), 1.74-1.86(\mathrm{~m}, 2 \mathrm{H}), 2.34-2.40(\mathrm{~m}, 1 \mathrm{H}), 3.20-3.24(\mathrm{~m}, 1 \mathrm{H}), 3.38-3.47(\mathrm{~m}, 2 \mathrm{H})$, $3.82(\mathrm{~s}, 3 \mathrm{H}), 4.13-4.18(\mathrm{~m}, 2 \mathrm{H}), 4.24-4.32(\mathrm{~m}, 1 \mathrm{H}), 8.34-8.42$ (br s, $1 \mathrm{H}), 11.43-11.47(\mathrm{br} \mathrm{s}, 1 \mathrm{H}) .{ }^{13} \mathrm{C}-$ NMR $\left(126 \mathrm{MHz}, \mathrm{CDCl}_{3}\right): \delta(\mathrm{ppm})=24.7,27.6,27.9,51.4,61.3,66.3,68.8,72.8,74.1,78.0,82.8,151.1$, 152.1, 155.2, 163.1, 173.1, 173.2. NMR spectra contain EtOAc signals. HRMS (ESI) m/z calc for $\mathrm{C}_{29} \mathrm{H}_{52} \mathrm{~N}_{4} \mathrm{O}_{11}[\mathrm{M}+\mathrm{H}]^{+} 633.3711$ found 633.3706 . 
$(2 R, 3 R, 5 S, 6 R)-N 1, N 10, N 11$-tri-Boc-methyl-6-amino-9-guanidino-2,3,5-trihydroxy-6,7$\mathrm{N}, \mathrm{O}$ - dimethyloxazolidine-2,3-0,O-dimethyloxazolidine nonanoate (48)

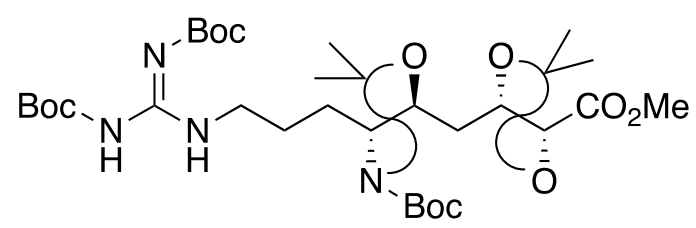

Diol 47 (270 mg, $0.427 \mathrm{mmol}, 1.00$ eq.) was dissolved in $8 \mathrm{~mL}$ of dry DCM and 2,2-dimethoxypropane (669 $\mu \mathrm{L}, 4.27 \mathrm{mmol}, 10.0$ eq.) was added. The solution was cooled to $0^{\circ} \mathrm{C}$ and $p \mathrm{TsOH}(10.0 \mathrm{mg}, 42.7$ $\mu \mathrm{mol}, 0.100$ eq.) was added. The mixture was stirred for $24 \mathrm{~h}$ at reflux and consequently was treated with $1 \mathrm{~mL}$ of DIPEA. The solvents were removed under reduced pressure and the residue purified with column chromatography $\left(\mathrm{SiO}_{2}\right.$, Hex:EtOAc - 3:1) to give the diacetonid $48(224 \mathrm{mg}, 78 \%)$ as a colorless solid. ${ }^{1} \mathrm{H}-\mathrm{NMR}\left(400 \mathrm{MHz}, \mathrm{CDCl}_{3}\right): \delta(\mathrm{ppm})=1.44(\mathrm{~s}, 9 \mathrm{H}), 1.47(\mathrm{~s}, 9 \mathrm{H}), 1.48(\mathrm{~s}, 9 \mathrm{H}), 1.48(\mathrm{~s}, 6 \mathrm{H}), 1.48$ (s, $6 \mathrm{H}), 1.58-1.68(\mathrm{~m}, 3 \mathrm{H}), 1.86-1.97(\mathrm{~m}, 2 \mathrm{H}), 3.37-3.47(\mathrm{~m}, 2 \mathrm{H}), 3.77(\mathrm{~s}, 3 \mathrm{H}), 4.14-4.20(\mathrm{~m}, 2 \mathrm{H})$, 4.27-4.36 (m, $1 \mathrm{H}$ ), 8.30-8.42 (br s, $1 \mathrm{H}), 11.43-11.47$ (br s, $1 \mathrm{H}) .{ }^{13} \mathrm{C}-\mathrm{NMR}\left(100 \mathrm{MHz}, \mathrm{CDCl}_{3}\right): \delta$ (ppm) $=17.5,18.8,25.8,27.2,28.1,28.4,28.6,52.5,54.0,58.7,58.9,60.5,73.7,79.4,92.6,93.1,111.0$, 126.1, 128.7, 139.7, 152.0, 153.4, 170.8. NMR spectra contain EtOAc signals. HRMS (ESI) m/z calc for $\mathrm{C}_{32} \mathrm{H}_{56} \mathrm{~N}_{4} \mathrm{O}_{11}[\mathrm{M}+\mathrm{H}]^{+} 673.4024$ found 673.4021 .

(2R,3S,5S,6R)-N1,N10,N11-tri-Boc-6-amino-9-guanidino-2,3,5-trihydroxy-6,7-N,Odimethyloxazolidine-2,3-0,0-dimethyloxazolidine nonanoic acid (49)

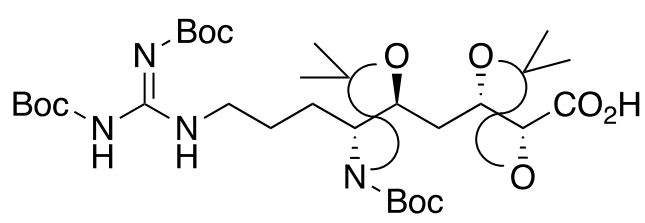

Methylester 48 (220 mg, 0.327 mmol, 1.00 eq.) was dissolved in $\mathrm{MeOH}$, THF and $\mathrm{H}_{2} \mathrm{O}, 2 \mathrm{~mL}$ each and cooled to $0{ }^{\circ} \mathrm{C}$ before $\mathrm{LiOH}(19.7 \mathrm{mg}, 0.818 \mathrm{mmol}, 2.50 \mathrm{eq}$.) was added. The solution was stirred for 10 min at $0{ }^{\circ} \mathrm{C}$ and at room temperature for $2 \mathrm{~h}$. To the mixture were added EtOAc and $\mathrm{H}_{2} \mathrm{O}$. The phases were separated and the aqueous phase was acidified with $0.5 \mathrm{M} \mathrm{HCl}$ and extracted with EtOAc three more times. The combined organic phase was washed with brine, dried over $\mathrm{Na}_{2} \mathrm{SO}_{4}$, filtered and the volatiles were removed under reduced pressure to give the free acid 49 (196 mg, 91\%) as a colorless solid. ${ }^{1} \mathrm{H}-\mathrm{NMR}\left(500 \mathrm{MHz}, \mathrm{CDCl}_{3}\right.$ ): $\delta(\mathrm{ppm})=1.47(\mathrm{~s}, 9 \mathrm{H}), 1.47(\mathrm{~s}, 9 \mathrm{H}), 1.47(\mathrm{~s}, 9 \mathrm{H}), 1.48(\mathrm{~s}, 6 \mathrm{H}), 1.48$ $(\mathrm{s}, 6 \mathrm{H}), 1.58-1.68(\mathrm{~m}, 4 \mathrm{H}), 1.83-1.94(\mathrm{~m}, 1 \mathrm{H}), 3.32-3.50(\mathrm{~m}, 2 \mathrm{H}), 3.82-3.85(\mathrm{~m}, 1 \mathrm{H}), 3.98-4.03(\mathrm{~m}, 1$ $\mathrm{H}), 4.17-4.25(\mathrm{~m}, 2 \mathrm{H}), 4.25-4.35(\mathrm{~m}, 1 \mathrm{H}), 8.35-8.43$ (br s, $1 \mathrm{H})$. NMR spectra contains EtOAc signals. HRMS (ESI) $\mathrm{m} / \mathrm{z}$ calc for $\mathrm{C}_{31} \mathrm{H}_{54} \mathrm{~N}_{4} \mathrm{O}_{11}[\mathrm{M}+\mathrm{H}]^{+} 659.3867$ found 659.3862 . 


\subsection{Preparation of protected (3S,5S,6S)-Galantinic acid (Gla) (40)}

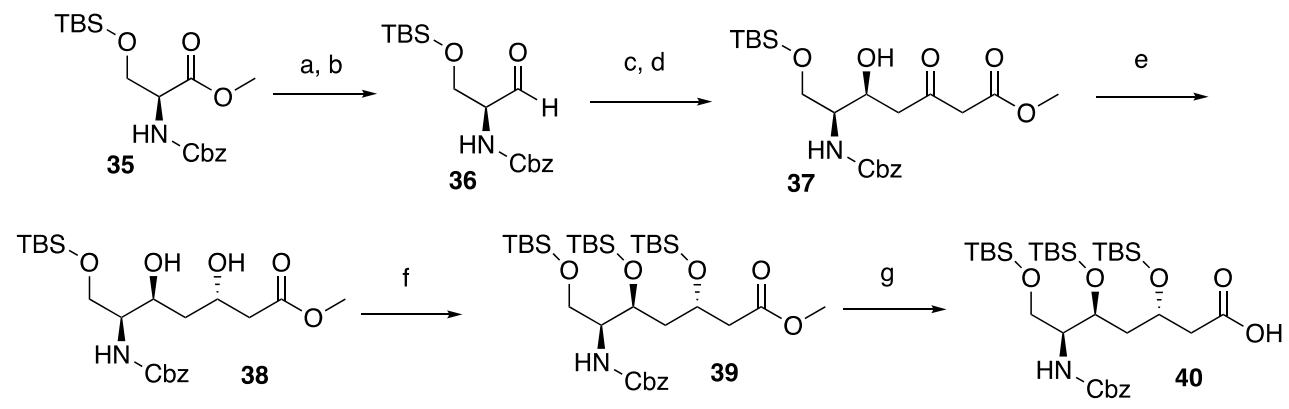

Scheme S2: Synthesis of (3S,5S,6S)-Galantinic acid (40):

a) $\mathrm{LiBH}_{4}, \mathrm{Et}_{2} \mathrm{O}, \mathrm{MeOH}$, reflux, $3 \mathrm{~h}, 97 \%$; b) $\mathrm{SO}_{3}{ }^{*} \mathrm{Pyr}$, DMSO, DIPEA, $0{ }^{\circ} \mathrm{C}, 1 \mathrm{~h}$; c) 2,2-dimethyl-4-methylene-6[(trimethylsilyl)oxy]-4H-1,3-dioxin, Eu(fod) $)_{3}, \mathrm{DCM}, 0{ }^{\circ} \mathrm{C}$ to rt, $24 \mathrm{~h}, 44 \%$; d) $\mathrm{MeOH}$, toluene, reflux, $4 \mathrm{~h}, 82 \%$; e) $\mathrm{Me}_{4} \mathrm{NBH}(\mathrm{OAc})_{3}, \mathrm{MeCN}, \mathrm{AcOH},-35{ }^{\circ} \mathrm{C}, 60 \mathrm{~h}, 86 \%$; f) TBSOTf, 2,6-lutidine, DCM, $-20{ }^{\circ} \mathrm{C}, 1.5 \mathrm{~h}, 57 \%$; g) LiOH, $\mathrm{MeOH}, \mathrm{THF}, \mathrm{H}_{2} \mathrm{O}$, rt, $48 \mathrm{~h}$.

\section{Cbz-L-Ser(TBDMS)-CHO (36)}<smiles>O=C[C@H](CO[AsH3])NC(=O)O</smiles>

Cbz-Ser(TBDMS)-OMe (10.3 g, $24.9 \mathrm{mmol}, 1.00$ eq.) was dissolved in $250 \mathrm{~mL}$ of $\mathrm{Et}_{2} \mathrm{O}$. To the resulting solution was added $\mathrm{LiBH}_{4}\left(2 \mathrm{M}\right.$ in THF, $25.0 \mathrm{~mL}, 50.0 \mathrm{mmol}, 2.00$ eq.) at $0{ }^{\circ} \mathrm{C}$ followed by $4 \mathrm{~mL}$ of $\mathrm{MeOH}$. The solution was stirred under reflux for $3 \mathrm{~h}$. The reaction was cooled to $0{ }^{\circ} \mathrm{C}$ and sat. $\mathrm{NH}_{4} \mathrm{Cl}$ solution added. The aqueous phase was extracted with EtOAc three times and the combined organic phase was washed with $\mathrm{H}_{2} \mathrm{O}$ and brine, dried over $\mathrm{Na}_{2} \mathrm{SO}_{4}$, filtered and the volatiles were removed under reduced pressure. The crude material was filtered through a pad of silica (Hex:EtOAc - 10:1) and the alcohol Cbz-Ser(TBDMS)-OH (9.22 g, 97\%) was obtained as a colorless oil and used directly in the next step.

The alcohol (4.00 g, $10.4 \mathrm{mmol}, 1.00$ eq.) was dissolved in $270 \mathrm{~mL} \mathrm{DCM}$ and at $0{ }^{\circ} \mathrm{C}$ DMSO $(31.1 \mathrm{~mL}$, $436 \mathrm{mmol}, 42.0$ eq.), DIPEA (28.3 mL, $166 \mathrm{mmol}, 16.0$ eq.) and $\mathrm{SO}_{3}{ }^{*}$ pyridine-complex (13.2 g, 83.0 mmol, 8.00 eq.) were added. The mixture was stirred for $1 \mathrm{~h}$ at $0{ }^{\circ} \mathrm{C}$ and then treated with sat. $\mathrm{NH}_{4} \mathrm{Cl}$ solution. The phases were separated, and the aqueous phase extracted with DCM three times. The combined organic phase was washed two times with $1 \mathrm{~N} \mathrm{HCl}$. After drying over $\mathrm{Na}_{2} \mathrm{SO}_{4}$, filtration and concentration under reduced pressure the resulting aldehyde 37 was used directly in the next step without further purification due to instability of the compound at prolonged time. ${ }^{1} \mathrm{H}-\mathrm{NMR}(500 \mathrm{MHz}$, $\left.\mathrm{CDCl}_{3}\right): \delta(\mathrm{ppm})=0.03(\mathrm{~s}, 3 \mathrm{H}), 0.04(\mathrm{~s}, 3 \mathrm{H}), 0.85(9 \mathrm{H}), 3.88(\mathrm{dd}, \mathrm{J}=9.9 \mathrm{~Hz}, \mathrm{~J}=3.8 \mathrm{~Hz}, 1 \mathrm{H}), 4.21$ (dd, J = $9.9 \mathrm{~Hz}, \mathrm{~J}=3.0 \mathrm{~Hz}, 1 \mathrm{H}), 4.32(\mathrm{~m}, 1 \mathrm{H}), 5.14(\mathrm{~s}, 2 \mathrm{H}), 5.62(\mathrm{~s}, 1 \mathrm{H}), 7.30-7.39(\mathrm{~m}, 5 \mathrm{H}), 9.66$ (s, $1 \mathrm{H}) .{ }^{13} \mathrm{C}$-NMR $\left(126 \mathrm{MHz}, \mathrm{CDCl}_{3}\right): \delta(\mathrm{ppm})=-5.3,-5.2,18.6,26.1,61.7,62.3,67.5,128.6,128.7,129.0$, 136.6, 156.5, 199.2. HRMS (ESI) $\mathrm{m} / \mathrm{z}$ calc for $\mathrm{C}_{17} \mathrm{H}_{27} \mathrm{NO}_{4} \mathrm{Si}[\mathrm{M}+\mathrm{H}]^{+} 338.1788$ found 338.1783 . 


\section{Benzyl ((2S,3S)-1-((tert-butyldimethylsilyl)oxy)-4-(2,2-dimethyl-4-oxo-4H-1,3-dioxin-6- yl)-3-hydroxybutan-2-yl)carbamate (37a)}

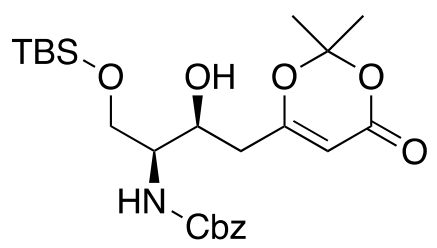

A solution of Cbz-L-Ser(TBDMS)-CHO (1.6 g, $4.75 \mathrm{mmol}, 1.00$ eq.) in $6 \mathrm{~mL}$ of DCM was cooled to $0{ }^{\circ} \mathrm{C}$ and $\mathrm{Eu}$ (fod) $)_{3}(1 \mathrm{~g}, 0.96 \mathrm{mmol}, 0.20$ eq. $)$ in $6 \mathrm{~mL}$ DCM was added. After 10 minutes of stirring at this temperature 2,2-dimethyl-4-methylene-6-[(trimethylsilyl)oxy]-4H-1,3-dioxin prepared by a literature method $^{X}(1.12 \mathrm{~g}, 5.23 \mathrm{mmol}, 1.10$ eq.) was added dropwise. The resulting solution was left stirring overnight at room temperature. DCM was removed in vacuo and residue was taken up in EtOAc, washed with $10 \%$ aqueous citric acid three times and with $\mathrm{NaHCO}_{3}$. Organic phase was dried over $\mathrm{Na}_{2} \mathrm{SO}_{4}$, filtered and the volatiles were removed under reduced pressure and the residue purified with column chromatography $\left(\mathrm{SiO}_{2}\right.$, gradient elution Hex:EtOAc - 4:1 to 1:1) to give the intermediate condensation product $37 \mathrm{a}(1.00 \mathrm{~g}, 44 \%)$ as a yellowish oil which crystallized to white solid over time. ${ }^{1} \mathrm{H}-\mathrm{NMR}\left(500 \mathrm{MHz}, \mathrm{CDCl}_{3}\right): \delta(\mathrm{ppm})=0.06(\mathrm{~s}, 3 \mathrm{H}), 0.07(\mathrm{~s}, 3 \mathrm{H}), 0.88(9 \mathrm{H}), 1.69(\mathrm{~s}, 6 \mathrm{H}), 2.33-2.45$ $(\mathrm{m}, 2 \mathrm{H}), 3.27-3.30(\mathrm{~m}, 1 \mathrm{H}), 3.62-3.67(\mathrm{~m}, 1 \mathrm{H}), 3.86-3.88(\mathrm{~m}, 2 \mathrm{H}), 4.26-4.30(\mathrm{~m}, 1 \mathrm{H}), 5.12(\mathrm{~s}, 2 \mathrm{H})$, 5.38-5.42 (m, $1 \mathrm{H})$, 7.30-7.39 (m, $5 \mathrm{H}) .{ }^{13} \mathrm{C}-\mathrm{NMR}\left(126 \mathrm{MHz}, \mathrm{CDCl}_{3}\right): \delta(\mathrm{ppm})=-5.6,18.2,24.8,25.4$, 25.9, 38.6, 54.3, 65.8, 67.1, 69.7, 95.4, 95.5, 106.7, 128.2, 128.3, 128.4, 128.4, 128.7, 136.4, 156.5, 161.1, 168.5. NMR spectra contain signals of EtOAc. HRMS (ESI) $\mathrm{m} / \mathrm{z}$ calc for $\mathrm{C}_{24} \mathrm{H}_{37} \mathrm{NO}_{7} \mathrm{Si}[\mathrm{M}+\mathrm{H}]^{+}$ 480.2418 found 480.2423 .

\section{Methyl (5S,6S)-6-(((benzyloxy)carbonyl)amino)-7-((tert-butyldimethylsilyl)oxy)-5- hydroxy-3-oxoheptanoate (37)}<smiles>COC(=O)CC(=O)CC(O)C(CO[As])NC(=O)OCc1ccccc1</smiles>

To a solution of $37 \mathrm{a}(1.00 \mathrm{~g}, 2.09 \mathrm{mmol}, 1.00$ eq.) in $16 \mathrm{~mL}$ of toluene methanol $(0.34 \mathrm{ml}, 8.36 \mathrm{mmol}$, 4.00 eq.) was added. The mixture was refluxed for 4 hours and consequently dried in vacuo. The residue was purified by flash chromatography $\left(\mathrm{SiO}_{2}\right.$, Hex:EtOAc - 3:1) to give $0.78 \mathrm{~g}(82 \%)$ of product 38 as a colorless oil. ${ }^{1} \mathrm{H}-\mathrm{NMR}\left(500 \mathrm{MHz}, \mathrm{CDCl}_{3}\right): \delta(\mathrm{ppm})=0.05(\mathrm{~s}, 3 \mathrm{H}), 0.06(\mathrm{~s}, 3 \mathrm{H}), 0.88(\mathrm{~s}, 9 \mathrm{H}), 2.65$ (dd, $\mathrm{J}=17.5,4.2 \mathrm{~Hz}, 1 \mathrm{H}$ ), $2.78(\mathrm{dd}, \mathrm{J}=17.5,8.6 \mathrm{~Hz}, 1 \mathrm{H}$ ), 3.33-3.38 (m, $1 \mathrm{H}), 3.47(\mathrm{br} \mathrm{s}, 1 \mathrm{H}), 3.62-3.66$ (m, $1 \mathrm{H}), 3.72(\mathrm{~s}, 3 \mathrm{H}), 3.78-3.81(\mathrm{~m}, 2 \mathrm{H}), 4.44-4.49(\mathrm{~m}, 1 \mathrm{H}), 5.11(\mathrm{br} \mathrm{s}, 1 \mathrm{H}), 5.32(\mathrm{~d}, \mathrm{~J}=9.4 \mathrm{~Hz}, 1$ H), 7.30-7.39 (m, $5 \mathrm{H}) .{ }^{13} \mathrm{C}-\mathrm{NMR}\left(126 \mathrm{MHz}, \mathrm{CDCl}_{3}\right): \delta(\mathrm{ppm})=-5.5,18.3,46.7,49.8,52.5,54.5,65.0$, 67.1, 68.0, 128.2, 128.7, 136.5, 156.6, 167.4, 202.4. HRMS (ESI) $\mathrm{m} / \mathrm{z}$ calc for $\mathrm{C}_{22} \mathrm{H}_{35} \mathrm{NO}_{7} \mathrm{Si}[\mathrm{M}+\mathrm{Na}]^{+}$ 476.2080 found 476.2075 . 
Methyl (3S,5S,6S)-6-(((benzyloxy)carbonyl)amino)-7-((tert-butyldimethylsilyl)oxy)-3,5dihydroxyheptanoate (38)<smiles>COC(=O)CC(O)CC(O)C(CO[As])NC(=O)OCc1ccccc1</smiles>

A solution of 38 (600 mg, $1.32 \mathrm{mmol}, 1.00$ eq.) in $4 \mathrm{~mL}$ of acetonitrile was cooled to $-35{ }^{\circ} \mathrm{C}$ and $\mathrm{Me}_{4} \mathrm{~N}(\mathrm{OAc})_{3} \mathrm{BH}\left(2.2 \mathrm{~g}, 9.24 \mathrm{mmol}, 7.00\right.$ eq.) preliminarly dissolved in $\mathrm{CH}_{3} \mathrm{CN} / \mathrm{AcOH}(4 \mathrm{~mL} / 4 \mathrm{~mL})$ was added. The reaction mixture was stirred at this temperature for 62 hours using an immersion cooler. It was then allowed to reach $0{ }^{\circ} \mathrm{C}$ and a saturated solution of $\mathrm{Na}-\mathrm{K}$-tartrate was added. The solution was stirred at this temperature for 4 hours. The phases were separated and the aqueous phase extracted 3 times with EtOAc. The combined organic layers were washed with brine, dried over $\mathrm{Na}_{2} \mathrm{SO}_{4}$, filtered and evaporated under reduced pressure to give $520 \mathrm{mg}(86 \%)$ of the crude product 39 . The NMR of this compound showed stereoselectivity of d.r. $=95: 5$ and sufficient purity, so the compound was directly used in the next step. ${ }^{1} \mathrm{H}-\mathrm{NMR}\left(400 \mathrm{MHz}, \mathrm{CDCl}_{3}\right): \delta(\mathrm{ppm})=0.04(\mathrm{~s}, 3 \mathrm{H}), 0.06(\mathrm{~s}, 3 \mathrm{H}), 0.87(\mathrm{~s}, 9 \mathrm{H})$, 1.50-1.58 (m, $1 \mathrm{H}), 1.66-1.75(\mathrm{~m}, 1 \mathrm{H}), 2.47-2.51(\mathrm{~m}, 2 \mathrm{H}), 3.01(\mathrm{br} \mathrm{s}, 1 \mathrm{H}), 3.58-3.63(\mathrm{~m}, 1 \mathrm{H}), 3.69(\mathrm{~s}$, $3 \mathrm{H}), 3.78-3.87(\mathrm{~m}, 2 \mathrm{H}), 4.24-4.33(\mathrm{~m}, 2 \mathrm{H}), 5.11(\mathrm{~s}, 2 \mathrm{H}), 5.45(\mathrm{~d}, \mathrm{~J}=9.0 \mathrm{~Hz}, 1 \mathrm{H}), 7.28-7.36(\mathrm{~m}, 5 \mathrm{H})$. ${ }^{13} \mathrm{C}-\mathrm{NMR}\left(100 \mathrm{MHz}, \mathrm{CDCl}_{3}\right): \delta(\mathrm{ppm})=-5.5,-5.5,18.2,25.9,40.0,41.4,51.1,55.1,65.3,66.1,67.0$, $69.7,128.2,128.2,128.3,128.6,128.7,136.6,156.7,173.3$. NMR spectra contain signals of EtOAc. HRMS (ESI) $\mathrm{m} / \mathrm{z}$ calc for $\mathrm{C}_{22} \mathrm{H}_{37} \mathrm{NO}_{7} \mathrm{Si}[\mathrm{M}+\mathrm{Na}]^{+} 478.2237$ found 478.2226 .

\section{Methyl (3S,5S,6S)-6-(((benzyloxy)carbonyl)amino)-3,5,7-tris((tert- butyldimethylsilyl)oxy)heptanoate (39)}

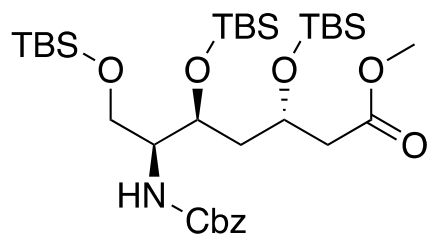

A solution of 39 (520 mg, $1.14 \mathrm{mmol}, 1.00$ eq.) in $6 \mathrm{~mL}$ of dry DCM 2,6-lutidine (4.56 mmol, 4.00 eq.) was added dropwise at $-20^{\circ} \mathrm{C}$. TBSOTf $(495 \mu \mathrm{L}, 2.85 \mathrm{mmol}, 2.50$ eq.) was added dropwise to the mixture, which was stirred at the same temperature for 1.5 hours then diluted with $\mathrm{Et}_{2} \mathrm{O}$ and washed successively two times with hydrochloric acid solution (0.1 M), sodium bicarbonate solution (5\%), saturated sodium chloride solution, dried over $\mathrm{Na}_{2} \mathrm{SO}_{4}$ and concentrated in vacuo. Flash chromatography (Hex/EtOAc - 5:95) gave the product $\mathbf{4 0}$ in $57 \%$ yield as a colorless oil (444 mg, 0.65 mmol). ${ }^{1} \mathrm{H}-\mathrm{NMR}\left(500 \mathrm{MHz}, \mathrm{CDCl}_{3}\right): \delta(\mathrm{ppm})=0.02(\mathrm{~s}, 3 \mathrm{H}), 0.03(\mathrm{~s}, 3 \mathrm{H}), 0.06(\mathrm{~s}, 3 \mathrm{H}), 0.08(\mathrm{~s}, 3 \mathrm{H})$, 0.09 (s, $3 \mathrm{H}), 0.10(\mathrm{~s}, 3 \mathrm{H}), 0.87(\mathrm{~s}, 9 \mathrm{H}), 0.88(\mathrm{~s}, 9 \mathrm{H}), 0.82(\mathrm{~s}, 9 \mathrm{H}), 1.56-1.67(\mathrm{~m}, 2 \mathrm{H}), 2.25-2.50(\mathrm{~m}$, $2 \mathrm{H}), 3.40-3.71(\mathrm{~m}, 4 \mathrm{H}), 3.65$ (s, $3 \mathrm{H}), 3.94-4.17(\mathrm{~m}, 2 \mathrm{H}), 4.95-5.10(\mathrm{~m}, 1 \mathrm{H}), 5.09(\mathrm{~s}, 2 \mathrm{H}), 7.29-7.39$ (m, $5 \mathrm{H}$ ). HRMS (ESI) $\mathrm{m} / \mathrm{z}$ calc for $\mathrm{C}_{34} \mathrm{H}_{65} \mathrm{NO}_{7} \mathrm{Si}_{3}[\mathrm{M}+\mathrm{Na}]^{+} 706.3967$ found 706.3956 (direct injection). 
(3S,5S,6S)-6-(((benzyloxy)carbonyl)amino)-3,5,7-tris((tert-

butyldimethylsilyl)oxy)heptanoic acid (40)

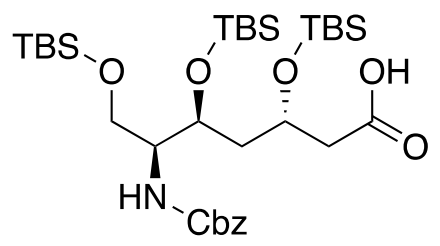

The methyl ester 40 (440 mg, 0.64 mmol, 1.00 eq.) was dissolved in $\mathrm{MeOH}$, THF and $\mathrm{H}_{2} \mathrm{O}, 3 \mathrm{~mL}$ each and cooled to $0{ }^{\circ} \mathrm{C}$ before $\mathrm{LiOH}(77 \mathrm{mg}, 3.20 \mathrm{mmol}, 5.00$ eq.) was added. The solution was stirred for $1 \mathrm{~h}$ at $0{ }^{\circ} \mathrm{C}$ and at room temperature for $48 \mathrm{~h}$. The volatiles were removed under reduced pressure and to the residue were added EtOAc and $\mathrm{H}_{2} \mathrm{O}$. The phases were separated and the aqueous phase was acidified with $0.5 \mathrm{M} \mathrm{HCl}$ and extracted three more times with EtOAc. The combined organic phases were washed with brine, dried over $\mathrm{Na}_{2} \mathrm{SO}_{4}$, filtered and the volatiles were removed under reduced pressure. The product 41 was introduces directly to the next step. ${ }^{1} \mathrm{H}-\mathrm{NMR}\left(500 \mathrm{MHz}, \mathrm{CDCl}_{3}\right): \delta(\mathrm{ppm})$ $=0.05(\mathrm{~s}, 3 \mathrm{H}), 0.06(\mathrm{~s}, 3 \mathrm{H}), 0.07(\mathrm{~s}, 3 \mathrm{H}), 0.07(\mathrm{~s}, 3 \mathrm{H}), 0.09(\mathrm{~s}, 3 \mathrm{H}), 0.09(\mathrm{~s}, 3 \mathrm{H}), 0.10(\mathrm{~s}, 3 \mathrm{H}), 0.86$ (s, $9 \mathrm{H}), 0.88(\mathrm{~s}, 9 \mathrm{H}), 0.88(\mathrm{~s}, 9 \mathrm{H}), 1.63-1.72(\mathrm{~m}, 1 \mathrm{H}), 1.83-1.93(\mathrm{~m}, 1 \mathrm{H}), 2.42(\mathrm{dd}, \mathrm{J}=15.2,6.2 \mathrm{~Hz}$, $1 \mathrm{H}), 2.57(\mathrm{dd}, \mathrm{J}=15.2,4.1 \mathrm{~Hz}, 1 \mathrm{H}), 3.43-3.52(\mathrm{~m}, 1 \mathrm{H}), 3.57-3.65(\mathrm{~m}, 2 \mathrm{H}), 4.02-4.08(\mathrm{~m}, 1 \mathrm{H}), 4.08-$ $4.16(\mathrm{~m}, 1 \mathrm{H}), 5.02(\mathrm{~m}, 1 \mathrm{H}), 5.09(\mathrm{~s}, 2 \mathrm{H}), 7.29-7.39(\mathrm{~m}, 5 \mathrm{H}) .{ }^{13} \mathrm{C}-\mathrm{NMR}\left(126 \mathrm{MHz}, \mathrm{CDCl}_{3}\right): \delta(\mathrm{ppm})=$ $-5.3,-5.2,-4.9,-4.8,-4.5,-4.1,18.0,18.2,18.2,25.8,25.9,26.0,41.5,41.6,54.5,61.3,66.6,66.8,67.1$, 128.4, 128.5, 128.7, 136.5, 156.3, 175.7. HRMS (ESI) $\mathrm{m} / \mathrm{z}$ calc for $\mathrm{C}_{33} \mathrm{H}_{63} \mathrm{NO}_{7} \mathrm{Si}_{3}[\mathrm{M}+\mathrm{Na}]^{+} 692.3810$ found 692.3816 . 


\subsection{Preparation of protected (3S,5R,6S)-Galantinic acid (Gla) (14)}

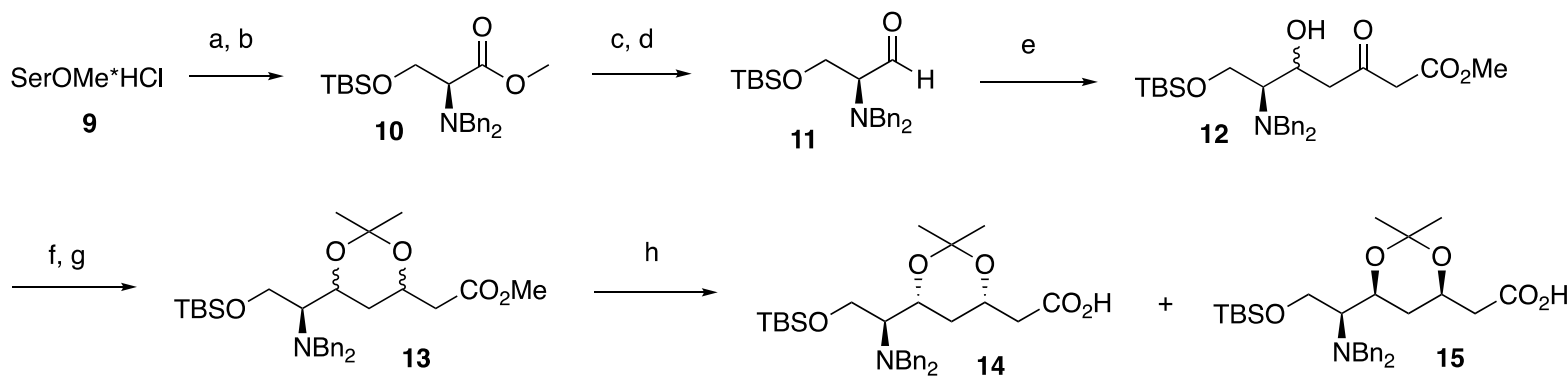

Scheme S3: Synthesis of (3S,5R,6S)-Galantinic acid (Gla) (14):

a) $\mathrm{BnBr}, \mathrm{NaHCO}_{3}$, THF, DMSO, reflux, $16 \mathrm{~h}, 99 \%$; b) TBDMSCl, imidazole, DCM, $50{ }^{\circ} \mathrm{C}, 12 \mathrm{~h}, 90 \%$; c) $\mathrm{LiBH}_{4}$, $\mathrm{Et}_{2} \mathrm{O}, \mathrm{MeOH}, 0{ }^{\circ} \mathrm{C}$ to reflux, $3 \mathrm{~h}, 97 \%$; d) $\mathrm{pyr}^{*} \mathrm{SO}_{3}$, DIPEA, DMSO, DCM, $0{ }^{\circ} \mathrm{C}$ to rt, $2 \mathrm{~h}, 99 \%$; e) methyl acetoacetate, $\mathrm{NaH}, n \mathrm{BuLi}$, THF, $-78{ }^{\circ} \mathrm{C}, 4 \mathrm{~h}, 80 \%$; f) $\mathrm{BEt}_{3}$, pivOH, $\mathrm{NaBH}_{4}, \mathrm{THF}, \mathrm{MeOH},-70{ }^{\circ} \mathrm{C}, 4 \mathrm{~h}, 95 \%$; g) 2,2-dmp, pTsOH, DCM, reflux, 12 h, $96 \%$; h) $\mathrm{LiOH}, \mathrm{MeOH} / \mathrm{H}_{2} \mathrm{O} / \mathrm{THF}, 0{ }^{\circ} \mathrm{C}$ to rt, $8 \mathrm{~h}, 70 \%$ (14), $11 \%$ (15).

\section{$\mathrm{Bn}_{2}$-L-Ser(TBDMS)-OMe (10)}<smiles>COC(=O)C(COC(C)(C)C)NC(=O)c1ccccc1</smiles>

H-L-Ser-OMe.HCl 9 (7.04 g, $45.3 \mathrm{mmol}, 1.00$ eq.) was dissolved in $30 \mathrm{~mL}$ DMSO and $120 \mathrm{~mL}$ of THF. At $0{ }^{\circ} \mathrm{C} \mathrm{NaHCO}_{3}(15.2 \mathrm{~g}, 181 \mathrm{mmol}, 4.00$ eq.) and benzyl bromide (16.1 mL, $136 \mathrm{mmol}, 3.00$ eq.) were added and the resulting suspension was stirred for $12 \mathrm{~h}$ under reflux. The reaction was cooled to room temperature and $300 \mathrm{~mL} \mathrm{H} \mathrm{H}_{2} \mathrm{O}$ were added. The aqueous phase was extracted with EtOAc three times and the combined organic phase was washed with brine, dried over $\mathrm{Na}_{2} \mathrm{SO}_{4}$, filtered and the volatiles were removed under reduced pressure. Further purification with column chromatography $\left(\mathrm{SiO}_{2}\right.$, Hex:EtOAc - 5:1) gave the dibenzylated ester (13.4 g, 99\%) as a yellow liquid. ${ }^{1} \mathrm{H}-\mathrm{NMR}(400 \mathrm{MHz}$, $\left.\mathrm{CDCl}_{3}\right): \delta(\mathrm{ppm})=2.41-2.58(\mathrm{~m}, 3 \mathrm{H}), 3.48(\mathrm{t}, \mathrm{J}=7.5 \mathrm{~Hz}, 1 \mathrm{H}), 3.57-3.63(\mathrm{~m}, 2 \mathrm{H}), 3.65-3.69(\mathrm{~m}, 2 \mathrm{H})$, $3.71(\mathrm{~s}, 3 \mathrm{H}), 3.79-3.87(\mathrm{~m}, 2 \mathrm{H}), 7.15-7.29(\mathrm{~m}, 10 \mathrm{H}) \cdot 7.29-7.15(\mathrm{~m}, 10 \mathrm{H}) .{ }^{13} \mathrm{C}-\mathrm{NMR}\left(100 \mathrm{MHz}, \mathrm{CDCl}_{3}\right)$ : $\delta(\mathrm{ppm})=51.4,54.8,59.3,61.8,65.2,126.9,127.4,127.5,128.5,129.0,138.6,140.9,171.7$. HRMS (ESI) $\mathrm{m} / \mathrm{z}$ calc $[\mathrm{M}+\mathrm{H}]+305.1594$ found 305.1596 .

$\mathrm{Bn}_{2}$-Ser-OMe (10.2 g, $34.1 \mathrm{mmol}, 1.00 \mathrm{eq}$ ) was dissolved in $200 \mathrm{~mL}$ of DCM. To the resulting solution imidazole (6.97 g, $102 \mathrm{mmol}, 3.00$ eq.) was added followed by TBDMSCl (7.70 g, $51.1 \mathrm{mmol}, 1.50$ eq.). The suspension was stirred under reflux for $12 \mathrm{~h}$. The reaction was cooled to room temperature and $300 \mathrm{~mL} \mathrm{H}_{2} \mathrm{O}$ were added. The aqueous phase was extracted with EtOAc three times and the combined organic phase was washed with brine, dried over $\mathrm{Na}_{2} \mathrm{SO}_{4}$, filtered and the volatiles were removed under reduced pressure. Further purification with column chromatography $\left(\mathrm{SiO}_{2}\right.$, Hex:EtOAc - 30:1) gave the TBDMS ether $10(12.68 \mathrm{~g}, 90 \%)$ as a colorless liquid. ${ }^{1} \mathrm{H}-\mathrm{NMR}\left(400 \mathrm{MHz}, \mathrm{CDCl}_{3}\right): \delta(\mathrm{ppm})=$ $0.00(\mathrm{~s}, 6 \mathrm{H}), 0.87(\mathrm{~s}, 9 \mathrm{H}), 3.56(\mathrm{t}, 1 \mathrm{H}, \mathrm{J}=6.0 \mathrm{~Hz}), 3.67-3.72(\mathrm{~m}, 2 \mathrm{H}), 3.76(\mathrm{~s}, 3 \mathrm{H}), 3.89$ (ABX, J = 10.3, $6.0 \mathrm{~Hz}, 1 \mathrm{H}), 3.93-3.99(\mathrm{~m}, 2 \mathrm{H}), 4.00(\mathrm{ABX}, \mathrm{J}=10.0,6.3 \mathrm{~Hz}, 1 \mathrm{H}), 7.20-7.43(\mathrm{~m}, 10 \mathrm{H}) .{ }^{13} \mathrm{C}-\mathrm{NMR}(100$ $\left.\mathrm{MHz}, \mathrm{CDCl}_{3}\right): \delta(\mathrm{ppm})=-5.7,-5.6,18.1,25.7,51.1,62.7,63.0,126.9,128.2,128.7,139.8,170.0$. HRMS (ESI) $\mathrm{m} / \mathrm{z}$ calc $[\mathrm{M}+\mathrm{H}]^{+} 414.2459$ found 414.2443 . 


\section{$\mathrm{Bn}_{2}$-L-Ser(TBDMS)-CHO (11)}<smiles>CC(C)(C)OCC(C=O)C(=O)c1ccccc1</smiles>

$\mathrm{Bn}_{2}$-Ser(TBDMS)-OMe 10 (10.3 g, $24.9 \mathrm{mmol}, 1.00$ eq.) was dissolved in $250 \mathrm{~mL}$ of $\mathrm{Et}_{2} \mathrm{O}$. To the resulting solution was added $\mathrm{LiBH}_{4}\left(2 \mathrm{M}\right.$ in THF, $25.0 \mathrm{~mL}, 50.0 \mathrm{mmol}, 2.00$ eq.) at $0{ }^{\circ} \mathrm{C}$ followed by 4 $\mathrm{mL}$ of $\mathrm{MeOH}$. The solution was stirred under reflux for $3 \mathrm{~h}$. The reaction was cooled to $0{ }^{\circ} \mathrm{C}$ and saturated $\mathrm{NH}_{4} \mathrm{Cl}$ solution added. The aqueous phase was extracted with EtOAc three times and the combined organic phase was washed with $\mathrm{H}_{2} \mathrm{O}$ and brine, dried over $\mathrm{Na}_{2} \mathrm{SO}_{4}$, filtered and the volatiles were removed under reduced pressure. The crude material was filtered through a pad of silica (Hex:EtOAc - 10:1) and the alcohol (9.31 g, 97\%) was obtained as a colorless oil. ${ }^{1} \mathbf{H}-\mathbf{N M R}(400 \mathrm{MHz}$, $\left.\mathrm{CDCl}_{3}\right): \delta(\mathrm{ppm})=0.10(\mathrm{~s}, 3 \mathrm{H}), 0.11(\mathrm{~s}, 3 \mathrm{H}), 0.94(\mathrm{~s}, 9 \mathrm{H}), 3.56(\mathrm{t}, 1 \mathrm{H}, \mathrm{J}=6.0 \mathrm{~Hz}), 2.92(\mathrm{~s} \mathrm{br}, 1 \mathrm{H}), 3.01$ (dt, J = 11.5, 5.8 Hz, 1H), 3.53-3.63 (m, 2H), 3.64-3.71 (m, 2H), 3.75 (ABX, J = 10.3, 5.8 Hz, 1H), 3.86 (ABX, J = 10.5, 6.0 Hz, 1H), 3.89-3.95 (m, 2H), 7.22-7.36 (m, 10H). ${ }^{13}$ C-NMR (100 MHz, CDCl $): \delta$ $(\mathrm{ppm})=-5.6,-5.6,18.1,25.9,54.1,59.6,59.8,60.9,127.1,128.4,128.9,139.6$. HRMS (ESI) m/z calc $[\mathrm{M}+\mathrm{H}]^{+} 386.2510$ found 386.2511 .

The later alcohol ( $4.00 \mathrm{~g}, 10.4 \mathrm{mmol}, 1.00 \mathrm{eq}$.) was dissolved in $270 \mathrm{~mL}$ DCM and at $0{ }^{\circ} \mathrm{C}$ DMSO (31.1 $\mathrm{mL}, 436 \mathrm{mmol}, 42.0$ eq.), DIPEA (28.3 mL, $166 \mathrm{mmol}, 16.0$ eq.) and $\mathrm{SO}_{3}{ }^{*}$ pyridine-complex (13.2 g, $83.0 \mathrm{mmol}, 8.00$ eq.) were added. The mixture was stirred for $1 \mathrm{~h}$ at $0{ }^{\circ} \mathrm{C}$ and then treated with saturated $\mathrm{NH}_{4} \mathrm{Cl}$ solution. The phases were separated, and the aqueous phase extracted with DCM three times. The combined organic phase was washed two times with $1 \mathrm{~N} \mathrm{HCl}$. After drying over $\mathrm{Na}_{2} \mathrm{SO}_{4}$, filtration and concentration under reduced pressure the resulting aldehyde 11 was used directly in the next step without further purification. Purification with column chromatography $\left(\mathrm{SiO}_{2}\right.$, Hex:EtOAc - 6:1) led to significant decomposition of the amino aldehyde. The compound is also unstable at prolonged time in $\mathrm{CHCl}_{3} .{ }^{1} \mathrm{H}-\mathrm{NMR}\left(400 \mathrm{MHz}, \mathrm{CDCl}_{3}\right): \delta(\mathrm{ppm})=0.10(\mathrm{~s}, 3 \mathrm{H}), 0.11(\mathrm{~s}, 3 \mathrm{H}), 0.93(\mathrm{~s}, 9 \mathrm{H}), 3.56(\mathrm{t}, 1 \mathrm{H}, \mathrm{J}=$ $6.0 \mathrm{~Hz}$ ), $2.92(\mathrm{~s} \mathrm{br}, 1 \mathrm{H}), 3.41(\mathrm{t}, \mathrm{J}=5.5 \mathrm{~Hz}, 1 \mathrm{H}), 3.88-3.93(\mathrm{~m}, 4 \mathrm{H}), 4.06(\mathrm{~d}, \mathrm{~J}=1.5 \mathrm{~Hz}, 1 \mathrm{H}), 4.08$ (d, J $=1.0 \mathrm{~Hz}, 1 \mathrm{H}), 7.22-7.44(\mathrm{~m}, 10 \mathrm{H}), 9.75(\mathrm{~s}, 1 \mathrm{H}) . \mathbf{H R M S}(\mathrm{ESI}) \mathrm{m} / \mathrm{z}$ calc $[\mathrm{M}+\mathrm{H}]^{+} 384.2353$ found 384.2363 .

\section{(5R/S,6S)-Methyl-7-((tert-butyldimethylsilyl)oxy)-6-(dibenzylamino)-5-hydroxy-3- oxoheptanoate (12)}

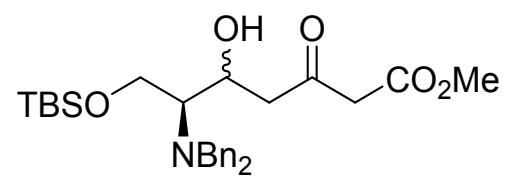

To a flame dried flask containing $\mathrm{NaH}(890 \mathrm{mg}, 37.1 \mathrm{mmol}, 3.60$ eq., washed with hexanes in advance) $73 \mathrm{~mL}$ of dry THF were added and the suspension was cooled to $0{ }^{\circ} \mathrm{C}$. To this suspension methyl acetoacetate ( $1.99 \mathrm{~mL}, 18.5 \mathrm{mmol}, 1.80$ eq.) was added dropwise and the suspension was stirred at this temperature for $20 \mathrm{~min}$ before $n \mathrm{BuLi}(2.5 \mathrm{M}$ in hexanes, $9.1 \mathrm{~mL}, 22.7 \mathrm{mmol}, 2.20$ eq.) was added dropwise at $0{ }^{\circ} \mathrm{C}$. After further $20 \mathrm{~min}$ the freshly prepared aldehyde $11(10.3 \mathrm{mmol}$ in $40 \mathrm{~mL}$ THF, 
1.00 eq.) was added slowly to the dienolate at $-78^{\circ} \mathrm{C}$. The resulting solution was stirred for further $3 \mathrm{~h}$ at $-78{ }^{\circ} \mathrm{C}$ before saturated $\mathrm{NH}_{4} \mathrm{Cl}$ solution was added. The THF was removed under reduced pressure and the aqueous phase was extracted three times with EtOAc. The combined organic phases were washed with brine, dried over $\mathrm{Na}_{2} \mathrm{SO}_{4}$, filtered and the volatiles were removed under reduced pressure. Further purification with column chromatography $\left(\mathrm{SiO}_{2}\right.$, Hex:EtOAc - 6:1) yielded the title compound 12 (4.07 $\mathrm{g}, 80 \%)$ as a 4:1 mixture of diastereomers as evaluated from HPLC-MS. ${ }^{1} \mathrm{H}-\mathrm{NMR}(400 \mathrm{MHz}$, $\left.\mathrm{CDCl}_{3}\right): \delta(\mathrm{ppm})=0.00(\mathrm{~s}, 3 \mathrm{H}), 0.01(\mathrm{~s}, 3 \mathrm{H}), 0.82(\mathrm{~s}, 9 \mathrm{H}), 1.49(\mathrm{~s}, 1 \mathrm{H}), 2.22-2.31(\mathrm{~m}, 1 \mathrm{H}), 2.53(\mathrm{dt}, \mathrm{J}=$ 8.8, $5.3 \mathrm{~Hz}, 1 \mathrm{H}), 3.00-3.07(\mathrm{~m}, 2 \mathrm{H}), 3.30(\mathrm{~d}, \mathrm{~J}=2.3 \mathrm{~Hz}, 2 \mathrm{H}), 3.46-3.51(\mathrm{~m}, 2 \mathrm{H}), 3.60(\mathrm{~s}, 3 \mathrm{H}), 3.72-3.78$ (m, 2H), $3.93(A B X, J=10.8,5.0 \mathrm{~Hz}, 2 \mathrm{H}), 4.20(\mathrm{tt}, \mathrm{J}=9.3,2.8 \mathrm{~Hz}, 1 \mathrm{H}), 7.09-7.23(\mathrm{~m}, 10 \mathrm{H}) .{ }^{13} \mathrm{C}-\mathrm{NMR}$ $\left(100 \mathrm{MHz}, \mathrm{CDCl}_{3}\right): \delta(\mathrm{ppm})=-5.6,-5.5,18.1,25.9,48.0,49.8,52.3,55.2,60.4,61.2,67.9,127.1,128.3$, 129.0, 139.7, 167.5, 203.7. HRMS (ESI) $\mathrm{m} / \mathrm{z}$ calc $[\mathrm{M}+\mathrm{H}]^{+} 500.2827$ found 500.2818.

(3R/S,5R/S,6S)-Methyl-7-((tert-butyldimethylsilyl)oxy)-6-(dibenzylamino)-3,5dihydroxy-3,5-0,0-acetonide-heptanoate (13)

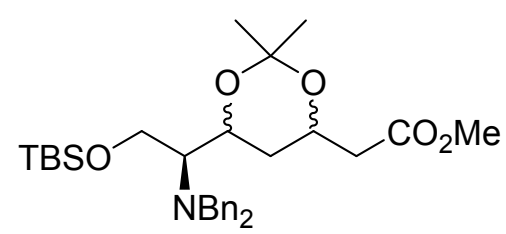

$\mathrm{BEt}_{3}(1 \mathrm{M}$ in THF, $3.06 \mathrm{~mL}, 3.06 \mathrm{mmol}, 1.50$ eq.) was dissolved in $2 \mathrm{~mL}$ of THF and $5.5 \mathrm{~mL}$ of $\mathrm{MeOH}$ and to this was added pivalic acid (10.4 $\mathrm{mg}, 0.102 \mathrm{mmol}, 5 \mathrm{~mol} \%)$. The solution was stirred for $1 \mathrm{~h}$ at room temperature and then cooled to $-70{ }^{\circ} \mathrm{C}$. $\beta$-keto ester $12(1.02 \mathrm{~g}, 2.04 \mathrm{mmol}, 1.00$ eq.) was added dropwise as a solution in $8 \mathrm{~mL}$ of THF followed by $\mathrm{NaBH}_{4}(233 \mathrm{mg}, 6.12 \mathrm{mmol}, 3.00$ eq.). After $4 \mathrm{~h}$ at $70{ }^{\circ} \mathrm{C}_{2} \mathrm{O}_{2}(15 \mathrm{~mL})$ and $\mathrm{H}_{2} \mathrm{O}(11 \mathrm{~mL})$ were added and the reaction could warm to room temperature. $\mathrm{H}_{2} \mathrm{O}$ and $\mathrm{CHCl}_{3}$ were added to the mixture and the phases were separated. The aqueous phase was extracted three more times with $\mathrm{CHCl}_{3}$ and the combined organic phases were washed with water $(5 \mathrm{x})$ and dried over $\mathrm{Na}_{2} \mathrm{SO}_{4}$. After filtration the volatiles were removed under reduced pressure and the crude product was further purified with column chromatography $\left(\mathrm{SiO}_{2}\right.$, Hex:EtOAc - 3:1) to give the corresponding diols (970 mg, 95\%) as a colorless wax. ${ }^{1} \mathrm{H}-\mathrm{NMR}\left(400 \mathrm{MHz}, \mathrm{CDCl}_{3}\right.$, partial signal doubling through diastereomeric mixture): $\delta(\mathrm{ppm})=0.09(\mathrm{~s}, 3 \mathrm{H}), 0.11(\mathrm{~s}, 3 \mathrm{H}), 0.90(\mathrm{~s}, 9 \mathrm{H}), 1.23-1.37$ (m, 1H), 2.06 (td, J = 14.3, 2.3 Hz, 1H), $2.46(A B X, J=15.6,7.8 \mathrm{~Hz}, 1 \mathrm{H}), 2.65$ (dt, J = 8.0, 5.3 Hz, 1H), 3.55-3.62 (m, 2H), 3.69 (s, 3H), 3.75-3.89 (m, 3H), 3.94-3.99 (m, 1H), 3.99-4.02 (m, 2H), 4.05-4.12 (m, $1 \mathrm{H}), 4.19-4.28(\mathrm{~m}, 1 \mathrm{H}), 7.19-7.32(\mathrm{~m}, 10 \mathrm{H}) .{ }^{13} \mathrm{C}-\mathrm{NMR}\left(100 \mathrm{MHz}, \mathrm{CDCl}_{3}\right.$, partial signal doubling through diastereomeric mixture): $\delta(\mathrm{ppm})=-5.6,-5.6,18.0,25.8,40.3,41.9,51.7,54.4,55.2,58.9,60.8,61.8$, 69.1, 72.9, 127.1, 128.3, 128.8, 139.7, 172.5. HRMS (ESI) $\mathrm{m} / \mathrm{z}$ calc $[\mathrm{M}+\mathrm{H}]^{+} 502.2983$ found 502.2968 .

The above diols (857 mg, $1.71 \mathrm{mmol}, 1.00$ eq.) were dissolved in $10 \mathrm{~mL}$ of DCM and $10 \mathrm{~mL}$ of 2,2-dimethoxy propane. To this solution was added $\mathrm{pTsOH}(33.0 \mathrm{mg}, 0.171 \mathrm{mmol}, 0.100$ eq.) and the reaction was stirred under reflux for $12 \mathrm{~h}$. After addition of $200 \mu \mathrm{l}$ of DIPEA all volatiles were removed under reduced pressure and the residue was further purified with column chromatography $\left(\mathrm{SiO}_{2}\right.$, Hex:EtOAc - 5:1) to yield the acetonide protected diols 3,5-syn-13 (888 mg, 96\%) as colorless oil. ${ }^{1} \mathbf{H}$ NMR $\left(400 \mathrm{MHz}, \mathrm{CDCl}_{3}\right.$, partial signal doubling through diastereomeric mixture): $\delta(\mathrm{ppm})=0.10(\mathrm{~s}, 3 \mathrm{H})$, 
$0.12(\mathrm{~s}, 3 \mathrm{H}), 0.96(\mathrm{~s}, 9 \mathrm{H}), 1.03(\mathrm{q}, \mathrm{J}=13.1 \mathrm{~Hz}, 1 \mathrm{H}), 1.29(\mathrm{~s}, 3 \mathrm{H}), 1.42(\mathrm{~s}, 3 \mathrm{H}), 1.59$ (s, 1H), 1.94 (dt, J = 13.3, $2.3 \mathrm{~Hz}, 1 \mathrm{H}), 2.38(\mathrm{ABX}, \mathrm{J}=15.3,5.8 \mathrm{~Hz}, 1 \mathrm{H}), 2.52(\mathrm{ABX}, \mathrm{J}=15.3,7.3 \mathrm{~Hz}, 1 \mathrm{H}), 2.65$ (dt, J = 8.0, $2.8 \mathrm{~Hz}, 1 \mathrm{H}), 3.66-3.75(\mathrm{~m}, 5 \mathrm{H}), 3.85(\mathrm{ABX}, \mathrm{J}=10.8,5.5 \mathrm{~Hz}, 1 \mathrm{H}), 3.89-3.95(\mathrm{~m}, 2 \mathrm{H}), 4.01$ (ABX, J $=10.8,2.5 \mathrm{~Hz}, 1 \mathrm{H}), 4.16(\mathrm{dt}, \mathrm{J}=11.0,2.3 \mathrm{~Hz}, 1 \mathrm{H}), 4.23-4.31(\mathrm{~m}, 1 \mathrm{H}), 7.19-7.40(\mathrm{~m}, 10 \mathrm{H}) .{ }^{13} \mathrm{C}-\mathrm{NMR}$ $\left(100 \mathrm{MHz}, \mathrm{CDCl}_{3}\right.$, partial signal doubling through diastereomeric mixture): $\delta(\mathrm{ppm})=-5.6,-5.5,18.2$, 19.7, 26.0, 30.0, 34.8, 41.5, 51.6, 55.6, 58.4, 62.2, 66.4, 67.1, 98.6, 126.8, 128.2, 128.7, 140.5, 171.5. HRMS (ESI) $\mathrm{m} / \mathrm{z}$ calc $[\mathrm{M}+\mathrm{H}]^{+} 542.3296$, found 542.3294 .

$(3 S, 5 R, 6 S)$-Galantinic acid (14) and (3R,5S,6S)-Galantinic acid (15)<smiles>CC(C)(C)OCC(N)CCCC(=O)O</smiles><smiles>CC(C)CCCCCC(N)COC(C)(C)C</smiles>

The methyl ester 13 ( $1.54 \mathrm{~g}, 2.85 \mathrm{mmol}, 1.00$ eq.) was dissolved in $\mathrm{MeOH}$, THF and $\mathrm{H}_{2} \mathrm{O}, 15 \mathrm{~mL}$ each and cooled to $0{ }^{\circ} \mathrm{C}$ before $\mathrm{LiOH} \cdot \mathrm{H}_{2} \mathrm{O}$ (198 mg, $5.70 \mathrm{mmol}, 2.00$ eq.) was added. The solution was stirred for $1 \mathrm{~h}$ at $0{ }^{\circ} \mathrm{C}$ and at room temperature for $4 \mathrm{~h}$. The volatiles were removed under reduced pressure and to the residue were added EtOAc and $\mathrm{H}_{2} \mathrm{O}$. The phases were separated and the aqueous phase was acidified with $0.5 \mathrm{M} \mathrm{HCl}$ and extracted three more times with EtOAc. The combined organic phases were washed with brine, dried over $\mathrm{Na}_{2} \mathrm{SO}_{4}$, filtered and the volatiles were removed under reduced pressure to yield the free acid $(1.22 \mathrm{~g}, 81 \%)$ as a colorless wax. Separation of the diastereomers was accomplished on this stage with column chromatography $\left(\mathrm{SiO}_{2}, 98.5: 1.5-\mathrm{DCM} / \mathrm{MeOH}\right)$ and the title compounds 214 ( $1.05 \mathrm{~g}, \mathbf{7 0} \%$ for $\mathbf{3 S , 5 R} \mathbf{- 1 4}$ and $170 \mathrm{mg}, 11 \%$ for $\mathbf{3 R}, \mathbf{5 S}-\mathbf{1 5})$ were obtained as colorless foams.

$(3 S, 5 R, 6 S)-14:{ }^{1} \mathrm{H}-\mathrm{NMR}\left(400 \mathrm{MHz}, \mathrm{CDCl}_{3}\right): \delta(\mathrm{ppm})=0.10(\mathrm{~s}, 3 \mathrm{H}), 0.13(\mathrm{~s}, 3 \mathrm{H}), 0.96(\mathrm{~s}, 9 \mathrm{H}), 1.05$ (q, $\mathrm{J}=12.8 \mathrm{~Hz}, 1 \mathrm{H}), 1.32(\mathrm{~s}, 3 \mathrm{H}), 1.42(\mathrm{~s}, 3 \mathrm{H}), 2.00(\mathrm{td}, \mathrm{J}=13.3,2.3 \mathrm{~Hz}, 1 \mathrm{H}), 2.47-2.57(\mathrm{~m}, 1 \mathrm{H}), 2.57-2.63$ (m, 1H), 3.68-3.74 (m, 2H), 3.84 (ABX, J = 10.8, $5.5 \mathrm{~Hz}, 1 \mathrm{H}), 3.90-3.97(\mathrm{~m}, 2 \mathrm{H}), 4.02(\mathrm{ABX}, \mathrm{J}=10.8$, $2.5 \mathrm{~Hz}, 1 \mathrm{H}), 4.17(\mathrm{dt}, \mathrm{J}=10.8,2.0 \mathrm{~Hz}, 1 \mathrm{H}), 4.23-4.31(\mathrm{~m}, 1 \mathrm{H}), 7.20-7.36(\mathrm{~m}, 10 \mathrm{H}) .{ }^{13} \mathrm{C}-\mathrm{NMR}(100 \mathrm{MHz}$, $\left.\mathrm{CDCl}_{3}\right): \delta(\mathrm{ppm})=-5.6,-5.5,18.1,19.7,26.0,29.9,34.6,41.2,55.7,58.2,62.1,66.2,66.9,99.0,126.8$, $128.2,128.7,140.4,175.3$.

(3R,5S,6S)-15: ${ }^{1} \mathrm{H}-\mathrm{NMR}\left(400 \mathrm{MHz}, \mathrm{CDCl}_{3}\right): \delta(\mathrm{ppm})=0.07(\mathrm{~s}, 3 \mathrm{H}), 0.07(\mathrm{~s}, 3 \mathrm{H}), 0.91(\mathrm{~s}, 9 \mathrm{H}), 1.14$ (td, $\mathrm{J}=13.1,2.0 \mathrm{~Hz}, 1 \mathrm{H}), 1.42(\mathrm{~s}, 3 \mathrm{H}), 1.46(\mathrm{~s}, 3 \mathrm{H}), 1.74(\mathrm{q}, \mathrm{J}=12.3 \mathrm{~Hz}, 1 \mathrm{H}), 2.46-2.53(\mathrm{~m}, 1 \mathrm{H}), 2.56-2.63$ (m, 2H), 3.68- $3.74(\mathrm{~m}, 2 \mathrm{H}), 3.62-3.70(\mathrm{~m}, 2 \mathrm{H}), 3.83-3.92(\mathrm{~m}, 2 \mathrm{H}), 4.01-4.09(\mathrm{~m}, 2 \mathrm{H}), 4.20(\mathrm{dt}, \mathrm{J}=11.5$, $2.3 \mathrm{~Hz}, 1 \mathrm{H}), 4.23-4.31(\mathrm{~m}, 1 \mathrm{H}), 7.21-7.38(\mathrm{~m}, 10 \mathrm{H}) .{ }^{13} \mathrm{C}-\mathrm{NMR}\left(100 \mathrm{MHz}, \mathrm{CDCl}_{3}\right): \delta(\mathrm{ppm})=-5.6,-5.5$, $18.1,19.3,25.9,30.1,33.3,41.3,55.8,60.2,61.2,66.0,69.0,99.0,126.6,128.0,129.1,140.9,175.0$.

HRMS (ESI) $\mathrm{m} / \mathrm{z}$ calc $[M+H]^{+} 528.3140$ found 528.3132 . 

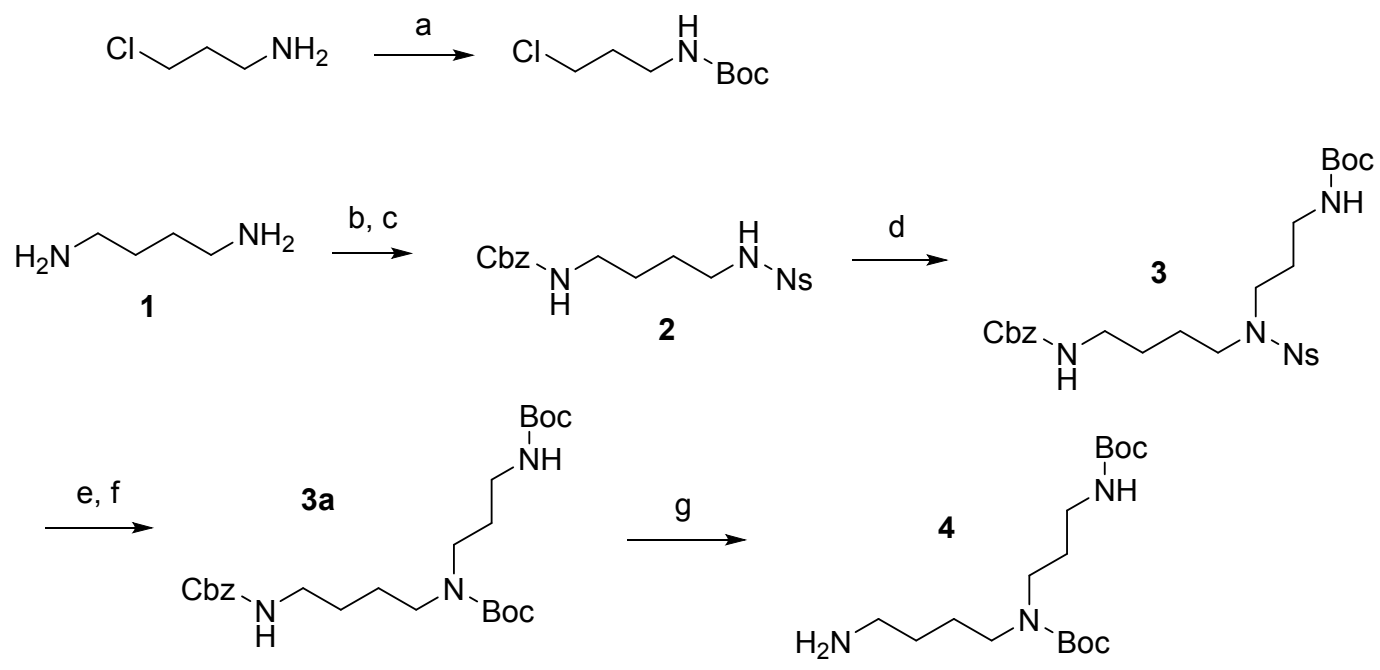

Scheme S4: Synthesis of protected spermidine (4):

a) $\mathrm{Boc}_{2} \mathrm{O}, \mathrm{Et}_{3} \mathrm{~N}, \mathrm{DCM}, 0^{\circ} \mathrm{C}$ to rt, $96 \%$; b) $\mathrm{CbzCl}$, chlorophorm, $0{ }^{\circ} \mathrm{C}$ to rt, $\left.16 \mathrm{~h} ; \mathrm{c}\right) \mathrm{oNsCl} \mathrm{Et}_{3} \mathrm{~N}, \mathrm{DCM}, \mathrm{rt}, 12 \mathrm{~h},(72 \%$ after 2 steps); d) N-Boc-3-chloroaminopropane, $\mathrm{K}_{2} \mathrm{CO}_{3}, \mathrm{KI}, \mathrm{DMF}, 80{ }^{\circ} \mathrm{C}, 12 \mathrm{~h}, 74 \%$; e) $\mathrm{PhSH}, \mathrm{K}_{2} \mathrm{CO}_{3}, \mathrm{DMF}, \mathrm{rt}, 3 \mathrm{~h}$ f) $\mathrm{Boc}_{2} \mathrm{O}, \mathrm{Et}_{3} \mathrm{~N}, \mathrm{DCM}$, rt, 4 h; g) $\mathrm{H}_{2}, \mathrm{Pd} / \mathrm{C}$, EtOAc, rt, $12 \mathrm{~h}$, (94\% after 3 steps).

\section{1-tert-butoxycarbonylamino-3-chloropropane}<smiles>CC(C)(C)OC(=O)NCCCCl</smiles>

3-Chloropropylamine (2.82 g, $30.3 \mathrm{mmol}, 1.00$ eq.) was dissolved in $70 \mathrm{~mL}$ of DCM and cooled to $0^{\circ} \mathrm{C}$. Next $\mathrm{Et}_{3} \mathrm{~N}$ (6.29 mL, $45.5 \mathrm{mmol}, 1.50$ eq.) and $\mathrm{Boc}_{2} \mathrm{O}$ (6.61 g, $30.3 \mathrm{mmol}, 1.00$ eq.) were added dropwise. The solution was stirred for $3 \mathrm{~h}$ at room temperature and the solvent was removed under reduced pressure. The residue was purified by column chromatography $\left(\mathrm{SiO}_{2}\right.$, Hex:EtOAc - 12:1) to give the title compound $(5.62 \mathrm{~g}, 96 \%)$ as a yellow oil. $\left.{ }^{1} \mathrm{H}-\mathrm{NMR}\left(400 \mathrm{MHz}^{\mathrm{CDCl}}\right)_{3}\right): \delta(\mathrm{ppm})=1.44(\mathrm{~s}, 9$ $\mathrm{H}), 1.96(\mathrm{~m}, 2 \mathrm{H}), 3.27(\mathrm{t}, \mathrm{J}=5.9 \mathrm{~Hz}, 2 \mathrm{H}), 3.58(\mathrm{t}, \mathrm{J}=6.5 \mathrm{~Hz}, 2 \mathrm{H}), 4.70(\mathrm{br} \mathrm{s}, 1 \mathrm{H}) .{ }^{13} \mathrm{C}-\mathrm{NMR}(100 \mathrm{MHz}$, $\left.\mathrm{CDCl}_{3}\right): \delta(\mathrm{ppm})=28.3,32.6,37.9,42.3,79.4,155.9$. HRMS (ESI) m/z calc for $\mathrm{C}_{8} \mathrm{H}_{16} \mathrm{CINO}_{2}[\mathrm{M}-\mathrm{tBu}+\mathrm{H}]^{+}$ 138.0316 found 138.0316 .

\section{Benzyloxycarbonyl-4-orthonitrobenzesulfonyl-diaminobutane (2)}<smiles>CCCCNNC(=O)OCc1ccccc1</smiles>

Diaminobutane 1 (10.0 g, $113 \mathrm{mmol}, 1.00$ eq.) was dissolved in $300 \mathrm{~mL}$ of $\mathrm{CHCl}_{3}$ and cooled to $0{ }^{\circ} \mathrm{C}$. $\mathrm{CbzCl}$ (3.27 mL, $22.7 \mathrm{mmol}, 0.20$ eq.) in $100 \mathrm{~mL}$ of $\mathrm{CHCl}_{3}$ was added dropwise over $3 \mathrm{~h}$ at this temperature. After addition the mixture was allowed to reach the room temperature and was stirred overnight. The organic phase was washed with brine three times and dried over $\mathrm{Na}_{2} \mathrm{SO}_{4}$, filtered and the solvent was removed under reduced pressure to give Cbz-protected diaminobutane as yellow oil, which was directly introduced in the next step. 
The above Cbz-diaminobutan was dissolved in $80 \mathrm{~mL}$ of DCM and cooled to $0{ }^{\circ} \mathrm{C}$ before $\mathrm{Et}_{3} \mathrm{~N}(4.72$ $\mathrm{mL}, 34.0 \mathrm{mmol}, 1.50$ eq.) and ortho-nitrobenzenesulfonyl chloride (5.52 g, $24.9 \mathrm{mmol}, 1.10 \mathrm{eq}$.) were added. The solution was stirred for $12 \mathrm{~h}$ at room temperature. The organic phase was washed two times with $1 \mathrm{~N} \mathrm{HCl}$ and with brine, was then dried over $\mathrm{Na}_{2} \mathrm{SO}_{4}$, filtered and the volatiles were removed under reduced pressure. Further purification with column chromatography $\left(\mathrm{SiO}_{2}\right.$, Hex:EtOAc - 2:1 to 1:1) yielded the nosylated product $2\left(6.65 \mathrm{~g}, 72 \%\right.$ over 2 steps) as an orange oil. ${ }^{1} \mathrm{H}-\mathrm{NMR}\left(400 \mathrm{MHz}, \mathrm{CDCl}_{3}\right)$ : $\delta(\mathrm{ppm})=1.61-1.49(\mathrm{~m}, 4 \mathrm{H}), 3.11(\mathrm{q}, \mathrm{J}=6.0 \mathrm{~Hz}, 2 \mathrm{H}), 3.17(\mathrm{q}, \mathrm{J}=6.0 \mathrm{~Hz}, 2 \mathrm{H}), 4.82$ (app t, J = 5.8 $\mathrm{Hz}, 1 \mathrm{H}), 5.07(\mathrm{~s}, 2 \mathrm{H}), 5.38(\mathrm{t}, \mathrm{J}=5.3 \mathrm{~Hz}, 1 \mathrm{H}), 7.38-7.32(\mathrm{~m}, 5 \mathrm{H}), 7.75-7.69(\mathrm{~m}, 2 \mathrm{H}), 7.87-7.83(\mathrm{~m}, 1$ $\mathrm{H}), 8.15-8.10(\mathrm{~m}, 1 \mathrm{H}) .{ }^{13} \mathrm{C}-\mathrm{NMR}\left(100 \mathrm{MHz}, \mathrm{CDCl}_{3}\right): \delta(\mathrm{ppm})=26.9,27.1,40.4,43.5,66.8,125.5,128.2$, $128.3,128.6,128.7,131.2,132.9,133.7,133.7,136.6,148.2,156.6$. NMR spectra contain signals of EtOAc. HRMS (ESI) m/z calc for $\mathrm{C}_{18} \mathrm{H}_{21} \mathrm{~N}_{3} \mathrm{O}_{6} \mathrm{~S}[\mathrm{M}+\mathrm{H}]^{+} 408.1224$ found 408.1218 .

\section{$N^{1}$-Cbz- $N^{4}$-oNs- $N^{8}$-Boc-spermidine (3)}<smiles>CC(C)(C)OC(=O)NCCCN(CCCCNC(=O)OCc1ccccc1)C(=O)O</smiles>

Nosylated amine 2 ( $3.51 \mathrm{~g}, 8.62 \mathrm{mmol}, 1.00$ eq.) was dissolved in $60 \mathrm{~mL}$ of DMF and $\mathrm{K}_{2} \mathrm{CO}_{3}(2.38 \mathrm{~g}$, $17.2 \mathrm{mmol}, 2.00$ eq.) and a catalytic amount of $\mathrm{KI}$ were added to the mixture. At $80{ }^{\circ} \mathrm{C} \mathrm{N}$-Boc-3chloroaminopropane $(2.50 \mathrm{~g}, 12.9 \mathrm{mmol}, 1.50$ eq.) was added as a solution in $20 \mathrm{~mL}$ of DMF. The reaction was stirred at $80{ }^{\circ} \mathrm{C}$ for $12 \mathrm{~h}$, cooled to room temperature and $100 \mathrm{~mL}$ of $\mathrm{H}_{2} \mathrm{O}$ were added. After extraction with EtOAc for three times ne combined organic phase was washed with brine, dried over $\mathrm{Na}_{2} \mathrm{SO}_{4}$, filtered and the volatiles were removed under reduced pressure. Further purification with column chromatography $\left(\mathrm{SiO}_{2}\right.$, Hex:EtOAc - 1:1) yielded the spermidine derivative $3(3.59 \mathrm{~g}, 74 \%)$ as a yellow wax. ${ }^{1} \mathrm{H}-\mathrm{NMR}\left(400 \mathrm{MHz}, \mathrm{CDCl}_{3}\right): \delta(\mathrm{ppm})=1.42(\mathrm{~s}, 9 \mathrm{H}), 1.43-1.51(\mathrm{~m}, 2 \mathrm{H}), 1.51-1.61(\mathrm{~m}, 2$ $\mathrm{H}), 1.72(\mathrm{t}, \mathrm{J}=6.5 \mathrm{~Hz}, 2 \mathrm{H}), 3.06-3.20(\mathrm{~m}, 2 \mathrm{H}), 3.30(\mathrm{dt}, \mathrm{J}=15.8,6.8 \mathrm{~Hz}, 4 \mathrm{H}), 4.78-4.96(\mathrm{br} \mathrm{s}, 1 \mathrm{H})$, 5.08 (s, $2 \mathrm{H})$, 7.28-7.38 (m, $5 \mathrm{H})$, 7.56-7.61 (m, $1 \mathrm{H})$, 7.61-7.70 (m, $2 \mathrm{H}), 7.92-8.00(\mathrm{~m}, 1 \mathrm{H}) .{ }^{13} \mathrm{C}-\mathrm{NMR}$ $\left(100 \mathrm{MHz}, \mathrm{CDCl}_{3}\right): \delta(\mathrm{ppm})=25.5,27.1,28.5,28.7,37.6,40.4,45.4,47.4,66.7,124.3,128.2,128.2$, $128.6,130.7,131.8,133.4,133.7,136.6,148.1,156.1,156.6$. NMR spectra contain signals of EtOAc. HRMS (ESI) $\mathrm{m} / \mathrm{z}$ calc for $\mathrm{C}_{26} \mathrm{H}_{36} \mathrm{~N}_{4} \mathrm{O}_{8} \mathrm{~S}[\mathrm{M}+\mathrm{H}]^{+} 565.2327$ found 565.2327 .

\section{$N^{1}-$ Cbz- $N^{4}, N^{8}$-bis-Boc-spermidine (3a)}<smiles>CC(C)(C)OC(=O)NCCCN(CCCCNC(=O)OCc1ccccc1)C(=O)OCc1ccccc1</smiles>

Spermidine derivative 3 ( $3.48 \mathrm{~g}, 6.16 \mathrm{mmol}, 1.00$ eq.) was dissolved in $75 \mathrm{~mL}$ of DMF and PhSH (1.29 $\mathrm{mL}, 12.3 \mathrm{mmol}, 2.00$ eq.) and $\mathrm{K}_{2} \mathrm{CO}_{3}(3.40 \mathrm{~g}, 24.7 \mathrm{mmol}, 4.00$ eq.) were added at room temperature. 
The mixture was stirred for $3 \mathrm{~h}$ before $150 \mathrm{~mL}$ of EtOAc were added. The organic phase was washed with $\mathrm{H}_{2} \mathrm{O}(2 \mathrm{x})$, with aq. sat. $\mathrm{NaHCO}_{3}(2 \mathrm{x})$ and brine. The organic phase was dried over $\mathrm{Na}_{2} \mathrm{SO}_{4}$ filtered and the solvent was removed under reduced pressure. The crude product was directly introduced in the next step.

The above residue was dissolved in $50 \mathrm{~mL}$ of DCM and cooled to $0{ }^{\circ} \mathrm{C}$ with an ice bath. To the solution was added $\mathrm{Et}_{3} \mathrm{~N}$ (1.74 mL, $12.3 \mathrm{mmol}, 2.00$ eq.) and $\mathrm{Boc}_{2} \mathrm{O}(2.1 \mathrm{~mL}, 9.25 \mathrm{mmol}, 1.50$ eq.) and the solution was stirred for $4 \mathrm{~h}$ at room temperature. All volatiles were removed under reduced pressure and the residue was further purified with column chromatography $\left(\mathrm{SiO}_{2}\right.$, Hex:EtOAc - 2:1) to yield spermidine derivative $3 \mathbf{a}\left(2.76 \mathrm{~g}, 94 \%\right.$ over 2 steps) as a colorless wax. ${ }^{1} \mathrm{H}-\mathrm{NMR}\left(400 \mathrm{MHz}, \mathrm{CDCl}_{3}\right): \delta$ $(\mathrm{ppm})=1.42(\mathrm{~s}, 9 \mathrm{H}), 1.44(\mathrm{~s}, 9 \mathrm{H}), 1.46-1.56(\mathrm{~m}, 4 \mathrm{H}), 1.58-1.68(\mathrm{~m}, 2 \mathrm{H}), 3.03-3.25(\mathrm{~m}, 8 \mathrm{H}), 5.08(\mathrm{~s}$, $2 \mathrm{H}), 7.28-7.38(\mathrm{~m}, 5 \mathrm{H}) .{ }^{13} \mathrm{C}-\mathrm{NMR}\left(100 \mathrm{MHz}, \mathrm{CDCl}_{3}\right): \delta(\mathrm{ppm})=25.8,27.4,28.6,37.7,40.8,43.9,46.6$, 66.7, 128.2, 128.6, 136.6, 156.2, 156.6. NMR spectra contain signals of EtOAc. HRMS (ESI) m/z calc for $\mathrm{C}_{25} \mathrm{H}_{41} \mathrm{~N}_{3} \mathrm{O}_{6}[\mathrm{M}+\mathrm{H}]^{+} 480.3068$ found 480.3072 .

\section{$N^{4}, N^{8}$-bis-Boc-spermidine (4)}<smiles>CC(C)(C)OC(=O)NCCCN(CCCCN)C(=O)O</smiles>

$N^{1}$-Cbz- $N^{4}, N^{8}$-bis-Boc-spermidine $3 a(2.70 \mathrm{~g}, 5.76 \mathrm{mmol}, 1.00$ eq.) was dissolved in $20 \mathrm{~mL}$ of EtOAc and $10 \% \mathrm{Pd} / \mathrm{C}(10 \mathrm{w} / \mathrm{w} . \%, 270 \mathrm{mg})$ was added in one portion. After flushing with $\mathrm{N} 2$ a balloon containing $\mathrm{H}_{2}$ gas was attached to the reaction flask. After stirring for $6 \mathrm{~h}$ at room temperature the flask was flushed with $\mathrm{N}_{2}$ and the solution was filtered through a pad of silica with several EtOAc washings. Evaporation under reduced pressure gave the free amine 4 (1,98 g, quant.) as colorless oil which was introduced directly in the next step. ${ }^{1} \mathrm{H}-\mathrm{NMR}\left(400 \mathrm{MHz}, \mathrm{DMSO}-\mathrm{d}_{6}\right): \delta(\mathrm{ppm})=1.27(\mathrm{~m}, 2 \mathrm{H}), 1.37(\mathrm{~s}, 9 \mathrm{H}), 1.38(\mathrm{~s}$, $9 \mathrm{H}), 1.40-1.50(\mathrm{~m}, 2 \mathrm{H}), 1.50-1.61(\mathrm{~m}, 2 \mathrm{H}), 2.52(\mathrm{t}, \mathrm{J}=7.0 \mathrm{~Hz}, 2 \mathrm{H}), 2.88$ (q, J = $6.8 \mathrm{~Hz}, 2 \mathrm{H}), 3.05-3.13$ (m, $4 \mathrm{H}), 6.78$ (br s, $1 \mathrm{H}) .{ }^{13} \mathrm{C}-\mathrm{NMR}(100 \mathrm{MHz}$, DMSO-d 6 ): $\delta(\mathrm{ppm})=28.1,28.2,30.6,37.6,41.5,44.0$, 44.3, 46.3, 46.5, 77.4, 78.2, 154.6, 155.6. HRMS (ESI) m/z calc for $\mathrm{C}_{17} \mathrm{H}_{35} \mathrm{~N}_{3} \mathrm{O}_{4}[\mathrm{M}+\mathrm{H}]+346.2700$ found 346.2707 . 


\subsection{Preparation of Cbz-L-mDAP(Boc)-OH (mDAP) (8)}

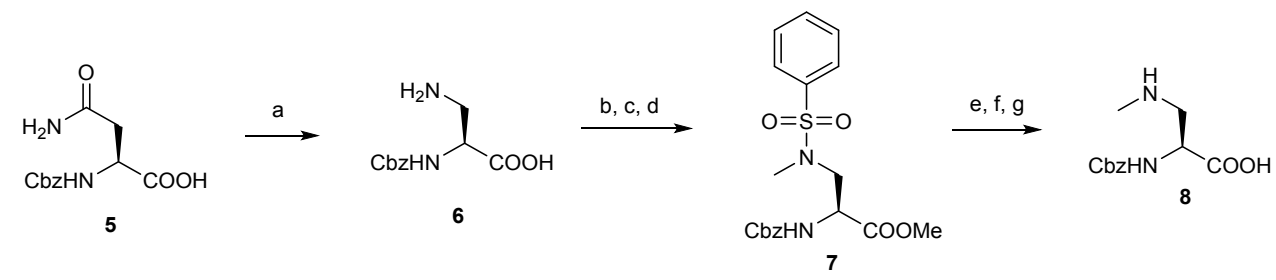

Scheme S5: Synthesis of Cbz-L-mDAP(Boc)-OH (8):

a) $\mathrm{Phl}(\mathrm{OAc})_{2}$, EtOAc, $\mathrm{MeCN}, \mathrm{H}_{2} \mathrm{O}, 15^{\circ} \mathrm{C}$ to rt, $4.5 \mathrm{~h}, 94 \%$; b) $\mathrm{SOCl}_{2}, \mathrm{MeOH}$, reflux, $2 \mathrm{~h}$; c) oNsCl, DCM, Et $\mathrm{Ot}_{3} \mathrm{~N}, \mathrm{rt}$, $12 \mathrm{~h}$; d) Mel, DMF, $\mathrm{K}_{2} \mathrm{CO}_{3}$, rt, $3 \mathrm{~h}$ (87\% over 3 steps); e) $\mathrm{LiOH}^{*} \mathrm{H}_{2} \mathrm{O}, \mathrm{MeOH}, \mathrm{THF}, \mathrm{H}_{2} \mathrm{O}, 0{ }^{\circ} \mathrm{C}, 12 \mathrm{~h}$; f) mercaptoethanol, DBU, DMF, rt, $3 \mathrm{~h}$; g) $\mathrm{Boc}_{2} \mathrm{O}, \mathrm{K}_{2} \mathrm{CO}_{3}, \mathrm{H}_{2} \mathrm{O}, 1,4$-dioxane, $0{ }^{\circ} \mathrm{C}$ to rt, $12 \mathrm{~h}$ (82\% over 3 steps).

\section{Cbz-L-DAP-OH (6)}<smiles>NC[C@H](NC(=O)OCc1ccccc1)C(=O)O</smiles>

Cbz-L-Asn-OH 5 (6.0 g, 22.6 mmol, 1.00 eq.) was dissolved in a mixture of EtOAc (60 mL), MeCN (70 $\mathrm{mL}$ ) and $\mathrm{H}_{2} \mathrm{O}(35 \mathrm{~mL})$. To the resulting suspension $\mathrm{Phl}(\mathrm{OAc})_{2}(8.72 \mathrm{~g}, 27.0 \mathrm{mmol}, 1.20$ eq.) was added in portions at $15{ }^{\circ} \mathrm{C}$. The suspension was stirred at this temperature for $30 \mathrm{~min}$ and then $4 \mathrm{~h}$ at room temperature. The white precipitate was filtered and washed with EtOAc several times yielding CbzDAP-OH $6(5.05 \mathrm{~g}, 94 \%)$ as a white solid which was introduced in the next step without further purification. ${ }^{1} \mathrm{H}-\mathrm{NMR}\left(500 \mathrm{MHz}, \mathrm{D}_{2} \mathrm{O} / \mathrm{DMSO}-\mathrm{d}_{6}+1 \% \mathrm{TFA}\right): \delta(\mathrm{ppm})=3.34(\mathrm{ABX}, \mathrm{J}=13.1,8.6 \mathrm{~Hz}, 1$ $\mathrm{H}), 3.56(\mathrm{ABX}, \mathrm{J}=13.1,5.4 \mathrm{~Hz}, 1 \mathrm{H}), 4.54(\mathrm{~m}, 1 \mathrm{H}), 5.18(\mathrm{~s}, 2 \mathrm{H}), 7.41-7.50(\mathrm{~m}, 5 \mathrm{H}) .{ }^{13} \mathrm{C}-\mathrm{NMR}(126$ $\mathrm{MHz}, \mathrm{D}_{2} \mathrm{O}+1 \%$ TFA): $\delta(\mathrm{ppm})=41.4,53.2,69.0,129.4,130.1,130.4,137.6,159.4,173.5$. HRMS (ESI) $\mathrm{m} / \mathrm{z}$ calc for $\mathrm{C}_{11} \mathrm{H}_{14} \mathrm{~N}_{2} \mathrm{O}_{4}[\mathrm{M}+\mathrm{H}]^{+} 239.1026$ found 239.1025 .

\section{Cbz-L-mDAP(oNs)-OMe (7)}<smiles>COC(=O)C(CN(C)S(=O)(=O)c1ccccc1[N+](=O)[O-])NC(=O)OCc1ccccc1</smiles>

Cbz-DAP-OH 6 (4.50 g, $18.9 \mathrm{mmol}, 1.00$ eq.) was suspended in $140 \mathrm{~mL}$ of methanol. At $0{ }^{\circ} \mathrm{C} \mathrm{SOCl}$ (1.80 $\mathrm{mL}, 24.6 \mathrm{mmol}, 1.30$ eq.) was added and the resulting solution stirred for $2 \mathrm{~h}$ under reflux. Repeated coevaporation with $\mathrm{MeOH}$ yielded the methyl ester as a yellow solid, which was introduced in the next step without further purification. 
The hydrochloride salt of the above ester $(4.82 \mathrm{~g}, 16.7 \mathrm{mmol}, 1.00$ eq.) was suspended in $60 \mathrm{~mL}$ of DCM and triethylamine $(7.30 \mathrm{~mL}, 41.9 \mathrm{mmol}, 2.50$ eq.) was added. To the resulting solution oNosCl ( $3.71 \mathrm{~g}, 16.7 \mathrm{mmol}, 1.00$ eq.) was added and the solution stirred at room temperature for $12 \mathrm{~h}$. To the solution was added EtOAc and the organic phase was washed two times with $1 \mathrm{~N} \mathrm{HCl}$, two times with sat. $\mathrm{NaHCO}_{3}$ solution, brine and dried over $\mathrm{Na}_{2} \mathrm{SO}_{4}$. Filtration and evaporation of the volatiles gave $\mathrm{Cbz}-\mathrm{DAP}(\mathrm{Ns})-\mathrm{OMe}$ as a yellow oil which was directly introduced in the next step without further purification.

Cbz-L-DAP(oNs)-OMe (7.32 g, $16.7 \mathrm{mmol}, 1.00 \mathrm{eq}$.) was dissolved in $50 \mathrm{~mL}$ DMF. To the solution was added $\mathrm{K}_{2} \mathrm{CO}_{3}(6.93 \mathrm{~g}, 50.2 \mathrm{mmol}, 3.00$ eq.) and methyl iodide $(2.10 \mathrm{~mL}, 33.5 \mathrm{mmol}, 2.00$ eq.). The mixture was stirred for $3 \mathrm{~h}$ at room temperature before EtOAc was added. The organic phase was washed two times with $1 \mathrm{~N} \mathrm{HCl}$, two times with sat. $\mathrm{NaHCO}_{3}$ solution, brine and dried over $\mathrm{Na}_{2} \mathrm{SO}_{4}$. Column chromatography $\left(\mathrm{SiO}_{2}\right.$, Hex:EtOAc - 1:1) gave the title compound $7(7.42 \mathrm{~g}, 87 \%$ over three steps) as a yellow oil. ${ }^{1} \mathrm{H}-\mathrm{NMR}\left(500 \mathrm{MHz}, \mathrm{CDCl}_{3}\right): \delta(\mathrm{ppm})=2.94(\mathrm{~s}, 3 \mathrm{H}), 3.57(\mathrm{dd}, \mathrm{J}=15.0,5.2 \mathrm{~Hz}, 1$ H), $3.71(\mathrm{dd}, \mathrm{J}=15.0,8.0 \mathrm{~Hz}, 1 \mathrm{H}), 3.77(\mathrm{~s}, 3 \mathrm{H}), 4.61(\mathrm{~m}, 1 \mathrm{H}), 5.08-5.12(\mathrm{~m}, 2 \mathrm{H}), 5.60(\mathrm{~d}, \mathrm{~J}=8.4 \mathrm{~Hz}$, $1 \mathrm{H}), 7.29-7.36(\mathrm{~m}, 5 \mathrm{H}), 7.59(\mathrm{dd}, \mathrm{J}=5.9,3.5 \mathrm{~Hz}, 1 \mathrm{H}), 7.65(\mathrm{~m}, 2 \mathrm{H}), 7.97(\mathrm{dd}, \mathrm{J}=5.5,3.2 \mathrm{~Hz}, 1 \mathrm{H})$. ${ }^{13} \mathrm{C}-\mathrm{NMR}\left(126 \mathrm{MHz}, \mathrm{CDCl}_{3}\right): \delta(\mathrm{ppm})=35.2,51.5,52.5,52.9,67.3,124.3,124.5,128.2,128.7,131.2$, 131.8, 132.2, 134.0, 136.2, 148.1, 156.0, 170.5. HRMS (ESI) $\mathrm{m} / \mathrm{z}$ calc for $\mathrm{C}_{19} \mathrm{H}_{21} \mathrm{~N}_{3} \mathrm{O}_{8} \mathrm{~S}[\mathrm{M}+\mathrm{H}]^{+}$ 452.1122 found 452.1118 .

\section{Cbz-L-mDAP(Boc)-OH (8)}<smiles>CN(CC(NC(=O)OCc1ccccc1)C(=O)O)C(=O)O</smiles>

Cbz-L-mDAP(Nos)-OMe 7 (5.23 g, $11.6 \mathrm{mmol}, 1.00$ eq.) was dissolved in $30 \mathrm{~mL}$ of THF and $30 \mathrm{~mL}$ of $\mathrm{MeOH}$. $\mathrm{LiOH}^{*} \mathrm{H}_{2} \mathrm{O}\left(1.06 \mathrm{~g}, 23.2 \mathrm{mmol}, 2.00\right.$ eq.) and $30 \mathrm{~mL}$ of $\mathrm{H}_{2} \mathrm{O}$ were added at $0{ }^{\circ} \mathrm{C}$ and the mixture was stirred overnight. THF and $\mathrm{MeOH}$ were evaporated and the residue was extracted with MTBE three times. The aqueous phase was acidified to $\mathrm{pH}=1$ with $1 \mathrm{~N} \mathrm{HCl}$ and extracted with EtOAc three times. The organic phase was dried over $\mathrm{Na}_{2} \mathrm{SO}_{4}$, filtered and concentrated under reduced pressure to obtain the crude acid which was directly introduced in the next step.

The above acid was dissolved in $55 \mathrm{~mL}$ DMF and DBU (4.45 mL, $30.0 \mathrm{mmol}, 2.50$ eq.) and mercaptoethanol ( $4.13 \mathrm{~mL}, 58.0 \mathrm{mmol}, 5.00$ eq.) were added successively. The solution was stirred for $3 \mathrm{~h}$ before $250 \mathrm{~mL} \mathrm{H}_{2} \mathrm{O}$ were added. The resulting precipitate was filtered off and $\mathrm{H}_{2} \mathrm{O}$ and DMF were removed under reduced pressure.

The above residue was dissolved in $100 \mathrm{~mL}$ of $\mathrm{H}_{2} \mathrm{O}$ and $100 \mathrm{~mL}$ of 1,4-dioxane. $\mathrm{K}_{2} \mathrm{CO}_{3}(6.40 \mathrm{~g}, 46.4$ $\mathrm{mmol}, 4.00$ eq.) and $\mathrm{Boc}_{2} \mathrm{O}(5.00 \mathrm{~mL}, 23.2 \mathrm{mmol}, 2.00$ eq. $)$ were added at $0{ }^{\circ} \mathrm{C}$. The solution was stirred 
overnight and 1,4-dioxane was removed under reduced pressure. The residue was extracted with MTBE three times and the aqueous phase acidified to $\mathrm{pH}=2$ with $1 \mathrm{~N} \mathrm{HCl}$ and extracted with EtOAc three times. The organic phase was dried over $\mathrm{Na}_{2} \mathrm{SO}_{4}$, filtered and the solvent removed under reduced pressure. Lyophilization gave Cbz-mDAP(Boc)-OH 8 (3.35 g, 82\% over 3 steps) as a slightly yellow solid. ${ }^{1} \mathrm{H}-\mathrm{NMR}\left(500 \mathrm{MHz}, \mathrm{DMSO}-\mathrm{d}_{6}\right): \delta(\mathrm{ppm})=1.38(\mathrm{~s}, 9 \mathrm{H}), 2.78(\mathrm{~d}, \mathrm{~J}=9.1 \mathrm{~Hz}, 3 \mathrm{H}), 3.24-3.33(\mathrm{~m}, 1$ $\mathrm{H}), 3.60-3.66(\mathrm{~m}, 1 \mathrm{H}), 4.25-4.31(\mathrm{~m}, 1 \mathrm{H}), 5.04(\mathrm{~s}, 2 \mathrm{H}), 7.28-7.38(\mathrm{~m}, 5 \mathrm{H}) .{ }^{13} \mathrm{C}-\mathrm{NMR}(126 \mathrm{MHz}$, DMSO$\left.d_{6}\right): \delta(p p m)=27.9,49.5,52.8,60.6,65.5,78.8,127.6,128.3,136.9,154.5,155.1,156.0,172.1$. HRMS (ESI) $\mathrm{m} / \mathrm{z}$ calc for $\mathrm{C}_{17} \mathrm{H}_{24} \mathrm{~N}_{2} \mathrm{O}_{6}[\mathrm{M}-\mathrm{H}]^{-} 351.1562$ found 351.1552 . 


\subsection{Synthesis of the protected left-hand side (LHS) fragment of PamB2}

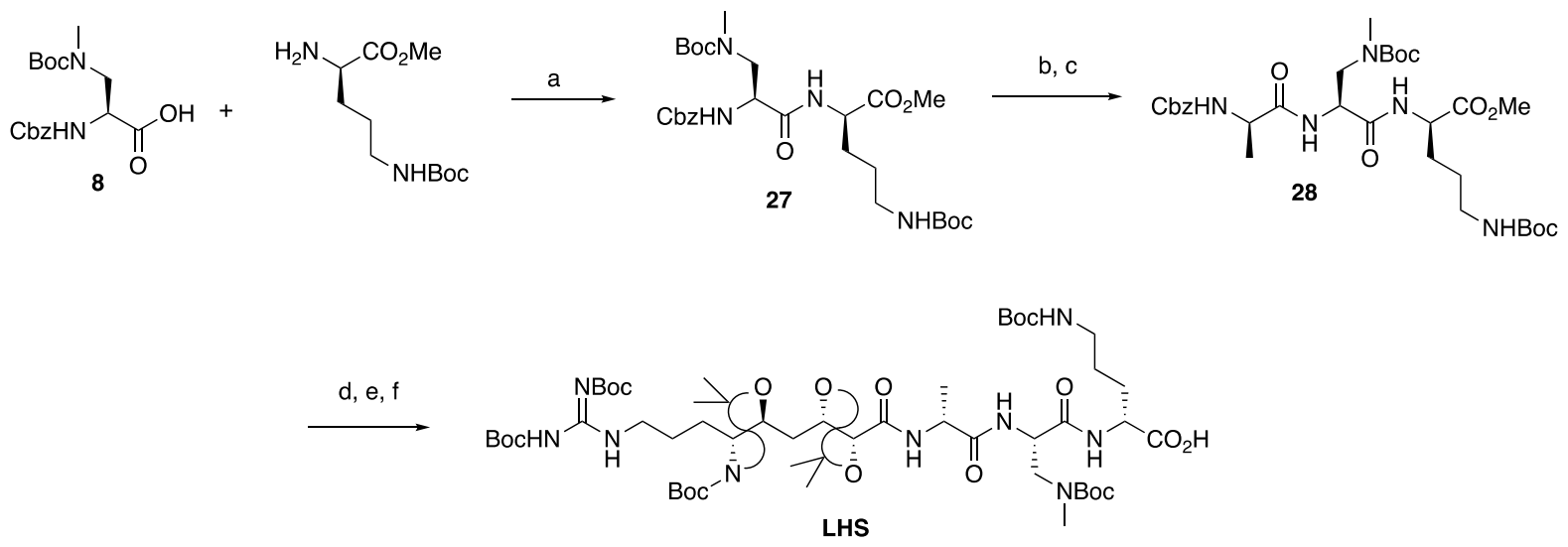

Scheme S6: Synthesis of the left-hand side fragment (LHS):

a) HATU, DIPEA, DMF, $0{ }^{\circ} \mathrm{C}$ to rt, $24 \mathrm{~h}, 86 \%$; b) $\mathrm{H}_{2}, \mathrm{Pd} / \mathrm{C}, \mathrm{MeOH} / \mathrm{EtOAc}(1: 1), \mathrm{rt}, 6 \mathrm{~h}, 99 \%$; c) Cbz-D-Ala-OH, EDC* $\mathrm{HCl}, \mathrm{HOBt}$, DIPEA, DCM, $0{ }^{\circ} \mathrm{C}$ to rt, 24 h, 85\%; d) $\mathrm{H}_{2}, \mathrm{Pd} / \mathrm{C}, \mathrm{MeOH} / \mathrm{EtOAc}(2: 1), \mathrm{rt}, 6 \mathrm{~h}, 99 \%$; e) Aga 49, EDC* $\mathrm{HCl}, \mathrm{HOBt}$, DIPEA, DCM, $0{ }^{\circ} \mathrm{C}$ to rt, $36 \mathrm{~h}, 94 \%$; f) $\mathrm{NaOH}, \mathrm{MeOH}, \mathrm{DCM}, 0{ }^{\circ} \mathrm{C}$ to rt, $1 \mathrm{~h}$, quant.

\section{Cbz-L-mDAP(Boc)-D-Orn(Boc)-OMe (27)}<smiles>CC(=O)OC(C)(C)OC(=O)NCCC[C@H](NC(=O)OCc1ccccc1)C(=O)N(C)CC(C)(C)C</smiles>

To a solution of Cbz-mDAP(Boc)-OH 8 ( $1.50 \mathrm{~g}, 4.27 \mathrm{mmol}, 1.05$ eq.) in $20 \mathrm{~mL}$ of DMF was added HATU $\left(1.70 \mathrm{~g}, 4.47 \mathrm{mmol}, 1.10 \mathrm{eq}\right.$.) at $0{ }^{\circ} \mathrm{C}$. The mixture was stirred for 5 minutes and then a solution of H-DOrn(Boc)-OMe (1.00 g, $4.06 \mathrm{mmol}, 1.00$ eq.) and DIPEA (1.08 mL, $6.09 \mathrm{mmol}, 1.50$ eq.) in $10 \mathrm{~mL}$ of DMF was added at $0{ }^{\circ} \mathrm{C}$. The mixture was stirred for $48 \mathrm{~h}$ at room temperature and was then diluted with EtOAc. The organic phase was washed with $1 \mathrm{~N} \mathrm{HCl}(4 \mathrm{x})$, sat. $\mathrm{NaHCO}_{3}$ solution (3x) and brine, dried over $\mathrm{Na}_{2} \mathrm{SO}_{4}$, filtered and the solvent was removed under reduced pressure. The residue was further purified by column chromatography $\left(\mathrm{SiO}_{2}\right.$, Hex:EtOAc - 1:1) to give the dipeptide product 27 $(2.02 \mathrm{~g}, 86 \%)$ as a colorless solid after lyophilization. ${ }^{1} \mathrm{H}-\mathrm{NMR}(500 \mathrm{MHz}$, DMSO-d6): $\delta(\mathrm{ppm})=1.33-$ 1-25 (m, $2 \mathrm{H}), 1.36(\mathrm{~s}, 9 \mathrm{H}), 1.36(\mathrm{~s}, 9 \mathrm{H}), 1.62-1.51(\mathrm{~m}, 1 \mathrm{H}), 1.74-1.63(\mathrm{~m}, 1 \mathrm{H}), 2.76(\mathrm{~d}, \mathrm{~J}=7.8 \mathrm{~Hz}, 3$ H), 2.89 (app. d, J = 6.0 Hz, 2 H), 3.44-3.34 (m, 2 H), 3.61 (s, 3 H), 5.03 (s, 2 H), 6.77 (br s, 1 H), 7.39$7.29(\mathrm{~m}, 5 \mathrm{H}), 8.45$ (s, $1 \mathrm{H}) .{ }^{13} \mathrm{C}-\mathrm{NMR}(126 \mathrm{MHz}$, DMSO-d6): $\delta(\mathrm{ppm})=172.4,170.1,155.9,155.7$, 137.0, 128.4, 127.8, 127.6, 78.9, 77.5, 65.6, 53.4, 51.9, 28.3, 28.0, 25.9. HRMS (ESI) m/z calc for $\mathrm{C}_{28} \mathrm{H}_{44} \mathrm{~N}_{4} \mathrm{O}_{9}[\mathrm{M}+\mathrm{H}]^{+} 581.3181$ found 581.3180 . 
<smiles>CC(=O)NCCC[C@H](NC(=O)[C@H](C)NC(=O)[C@H](C)NC(=O)OCc1ccccc1)C(=O)NCC(C)(C)C</smiles>

Cbz-mDAP(Boc)-D-Orn(Boc)-OMe 27 (848 mg, $1.46 \mathrm{mmol}, 1.00$ eq.) was dissolved in $5 \mathrm{~mL}$ of $\mathrm{MeOH} /$ EtOAc (1:1) and 10\% Pd/C (10 w/w.\%, $85 \mathrm{mg}$ ) was added in one portion. After flushing with $\mathrm{N}_{2}$ a balloon containing $\mathrm{H}_{2}$ was attached to the reaction flask. After stirring for $6 \mathrm{~h}$ at room temperature the flask was flushed with $\mathrm{N}_{2}$ and the solution was filtered through a pad of silica with several EtOAc washings. Evaporation under reduced pressure gave the free amine (652 mg, 99\%) as colorless oil which was introduced directly in the next step.

To a solution of Cbz-D-Ala-OH (391 mg, $1.75 \mathrm{mmol}, 1.20 \mathrm{eq}$.) and the above amine (1.46 mmol) in 7 $\mathrm{mL}$ of DCM was added DIPEA (382 $\mu \mathrm{L}, 2.19 \mathrm{mmol}, 1.5 \mathrm{eq}$.), EDC. $\mathrm{HCl}$ (294 mg, $1.53 \mathrm{mmol}, 1.05 \mathrm{eq}$.) and HOBt (207 mg, $1.53 \mathrm{mmol}, 1.05$ eq.) at $0{ }^{\circ} \mathrm{C}$. The mixture was stirred for $24 \mathrm{~h}$ at room temperature and was then diluted with EtOAc. The organic phase was washed with $1 \mathrm{~N} \mathrm{HCl}(2 x)$, sat. $\mathrm{NaHCO}_{3}$ solution (2x) and brine, dried over $\mathrm{Na}_{2} \mathrm{SO}_{4}$, filtered and the solvent was removed under reduced pressure. The residue was further purified by column chromatography $\left(\mathrm{SiO}_{2}, \mathrm{Hex}: \mathrm{EtOAc}-1: 2\right.$ to 1:3) to give the tripeptide $28(805 \mathrm{mg}, 85 \%)$ as colorless solid after lyophilization. ${ }^{1} \mathrm{H}-\mathrm{NMR}\left(500 \mathrm{MHz}, \mathrm{CDCl}_{3}\right)$ : $\delta(p p m)=1.37(\mathrm{~d}, \mathrm{~J}=7.0 \mathrm{~Hz}, 3 \mathrm{H}), 1.40(\mathrm{~s}, 9 \mathrm{H}), 1.46(\mathrm{~s}, 9 \mathrm{H}), 1.47-1.53(\mathrm{~m}, 2 \mathrm{H}), 1.55-1.63(\mathrm{~m}, 1 \mathrm{H})$, 1.81-1.89 (m, $1 \mathrm{H}), 2.87(\mathrm{~s}, 3 \mathrm{H}), 3.06(\mathrm{~m}, 2 \mathrm{H}), 3.56(\mathrm{~m}, 1 \mathrm{H}), 3.70(\mathrm{~s}, 3 \mathrm{H}), 3.73-3.80(\mathrm{~m}, 1 \mathrm{H}), 4.00-$ $4.08(\mathrm{~m}, 1 \mathrm{H}), 4.40-4.47(\mathrm{~m}, 1 \mathrm{H}), 4.51-4.58(\mathrm{~m}, 1 \mathrm{H}), 4.96-5.04(\mathrm{~m}, 1 \mathrm{H}), 5.11-5.14(\mathrm{~m}, 1 \mathrm{H}), 5.61(\mathrm{br}$ $\mathrm{s}, 1 \mathrm{H}), 7.28-7.36(\mathrm{~m}, 5 \mathrm{H}), 8.18$ (br s). ${ }^{13} \mathrm{C}-\mathrm{NMR}\left(126 \mathrm{MHz}, \mathrm{CDCl}_{3}\right): \delta(\mathrm{ppm})=17.4,25.7,28.6,29.3$, 36.3, 40.2, 50.2, 51.4, 52.0, 52.4, 67.2, 79.3, 80.9, 128.2, 128.6, 136.2, 156.4, 169.6, 172.2, 173.5 . NMR spectra contain signals of EtOAc. HRMS (ESI) m/z calc for $\mathrm{C}_{31} \mathrm{H}_{49} \mathrm{~N}_{5} \mathrm{O}_{10}[\mathrm{M}+\mathrm{H}]^{+} 652.3552$ found 652.3558 .

\section{$\mathrm{Boc}_{3}$-Agm-Hpa(diacetonid)-D-Ala-L-mDAP(Boc)-D-Orn(Boc)-OH (LHS)}

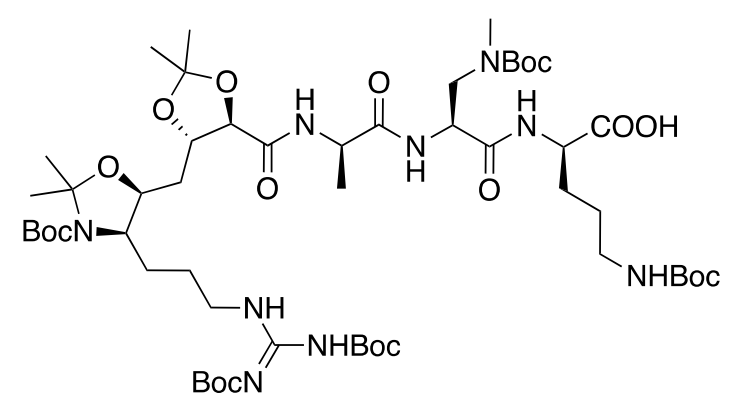


Cbz-D-Ala-mDAP(Boc)-D-Orn(Boc)-OMe 28 (778 mg, 1.19 mmol, 1.00 eq.) was dissolved in $15 \mathrm{~mL}$ MeOH:EtOAc (2:1) and 10\% Pd/C (10 w/w\%, $78 \mathrm{mg})$ was added in one portion. After flushing with $\mathrm{N}_{2}$ a balloon containing $\mathrm{H}_{2}$ was attached to the reaction flask. After stirring for $6 \mathrm{~h}$ at room temperature the flask was flushed with $\mathrm{N}_{2}$ and the solution was filtered through a pad of celite with several $\mathrm{MeOH}$ washings. Evaporation under reduced pressure gave the free amine (635 mg, 99\%) as colorless solid, which was introduced directly in the next step.

HRMS (ESI): $\mathrm{m} / \mathrm{z}=518.3183$ (calc. for $[\mathrm{M}+\mathrm{H}]^{+}: 518.3184$ )

H-D-Ala-mDAP(Boc)-D-Orn(Boc)-OMe (153 mg, $0.286 \mathrm{mmol}, 1.10$ eq.) and Aga 49 (177 mg, 0.269 mmol. 1.00 eq.) were dissolved in $3 \mathrm{~mL}$ of DCM and cooled to $0{ }^{\circ} \mathrm{C}$. To this mixture was added DIPEA (71.4 $\mu \mathrm{L}, 0.408 \mathrm{mmol}, 2.50$ eq.) followed by $\mathrm{EDC} \cdot \mathrm{HCl}(57.0 \mathrm{mg}, 0.296 \mathrm{mmol}, 1.10 \mathrm{eq}$ ) ) and $\mathrm{HOBt}(40.0$ $\mathrm{mg}, 0.296 \mathrm{mmol}, 1.10$ eq.). The mixture was stirred for $36 \mathrm{~h}$ at room temperature and was then diluted with EtOAc. The organic phase was washed with $1 \mathrm{~N} \mathrm{HCl}(2 \mathrm{x})$, sat. $\mathrm{NaHCO}_{3}$ solution $(2 \mathrm{x})$ and brine, dried over $\mathrm{Na}_{2} \mathrm{SO}_{4}$, filtered and the solvent was removed under reduced pressure. The residue was further purified by column chromatography $\left(\mathrm{SiO}_{2}, 98: 2-\mathrm{DCM} / \mathrm{MeOH}\right)$ to give the tetraptide $(290 \mathrm{mg}$, 94\%) as colorless solid after lyophilization. ${ }^{1} \mathrm{H}-\mathrm{NMR}\left(400 \mathrm{MHz}, \mathrm{CDCl}_{3}\right): \delta(\mathrm{ppm})=1.37$ (s, $\left.3 \mathrm{H}\right), 1.39$ (s, $9 \mathrm{H}), 1.40-1.53$ (s, $9 \mathrm{H}), 1.40-1.53$ (s, $9 \mathrm{H}), 1.40-1.53$ (s, $9 \mathrm{H}), 1.40-1.53$ (s, $9 \mathrm{H}), 1.40-1.53$ (s, $2 \mathrm{H})$, 1.40-1.53 (s, $6 \mathrm{H}), 1.40-1.53$ (s, $6 \mathrm{H}), 1.55-1.69(\mathrm{~m}, 4 \mathrm{H}), 1.71-1.91(\mathrm{~m}, 2 \mathrm{H}), 2.02 .-2.15(\mathrm{~m}, 2 \mathrm{H}), 2.20-$ $2.35(\mathrm{~m}, 1 \mathrm{H}), 2.85(\mathrm{~s}, 3 \mathrm{H}), 3.06(\mathrm{q}, \mathrm{J}=6.2 \mathrm{~Hz}, 2 \mathrm{H}), 3.32-3.43(\mathrm{~m}, 2 \mathrm{H}), 3.43-3.52(\mathrm{~m}, 1 \mathrm{H}), 3.69(\mathrm{~s}, 3$ H), 3.73-3.82 (m, $1 \mathrm{H}), 3.96-4.04(\mathrm{~m}, 1 \mathrm{H}), 4.12-4.32(\mathrm{~m}, 3 \mathrm{H}), 4.37-4.45(\mathrm{~m}, 1 \mathrm{H}), 4.45-4.53(\mathrm{~m}, 1 \mathrm{H})$, 4.90-5.00 (m, $1 \mathrm{H}), 7.02(\mathrm{~d}, \mathrm{~J}=6.0 \mathrm{~Hz}, 1 \mathrm{H}), 7.28-7.39(\mathrm{~m}, 1 \mathrm{H}), 7.95-8.05(\mathrm{br} \mathrm{s}, 1 \mathrm{H}), 8.24-8.42(\mathrm{br} \mathrm{s}$, $1 \mathrm{H}), 11.44(\mathrm{~s}, 1 \mathrm{H}) .{ }^{13} \mathrm{C}-\mathrm{NMR}\left(100 \mathrm{MHz}, \mathrm{CDCl}_{3}\right): \delta(\mathrm{ppm})=17.7,23.7,25.2,25.9,26.1,27.3,27.6$, 27.7, 28.1, 28.4, 28.4, 28.5, 28.9, 36.1, 40.0, 50.3, 52.1, 52.4, 53.5, 55.4, 58.7, 59.0, 73.8, 79.0, 79.7, $78.0,80.8,92.4,92.9,110.6,152.8,153.3,156.1,156.2,158.2,169.4,172.2,172.6$. NMR spectra contain signals of EtOAc. HRMS (ESI) $\mathrm{m} / \mathrm{z}$ calc for $\mathrm{C}_{54} \mathrm{H}_{95} \mathrm{~N}_{9} \mathrm{O}_{18}[\mathrm{M}+\mathrm{H}]^{+} 1158.6868$ found 1158.6875 .

The tetrapeptide was dissolved in DCM:MeOH 9:1 (3 mL) and cooled to $0{ }^{\circ} \mathrm{C}$. Next, $2 \mathrm{M} \mathrm{NaOH}$ in $\mathrm{MeOH}$ (152 $\mu \mathrm{L}, 0.304 \mathrm{mmol}$ ) was added. The mixture was stirred for $1 \mathrm{~h}$ at room temperature before TLC control was conducted, then the volatiles were removed under reduced pressure. The residue was taken up with water and EtOAc. The phases were separated, and the aqueous phase was carefully acidified to $\mathrm{pH}=2$ with $0.5 \mathrm{~N} \mathrm{HCl}$ and then extracted three times with EtOAc. The combined organic phases were washed with brine, dried over $\mathrm{Na}_{2} \mathrm{SO}_{4}$, filtered and the solvent was removed under reduced pressure to quantatively yield the free acid LHS, which was then introduced to the next step. 


\subsection{Synthesis of the right-hand side (RHS) fragment of PamB2}

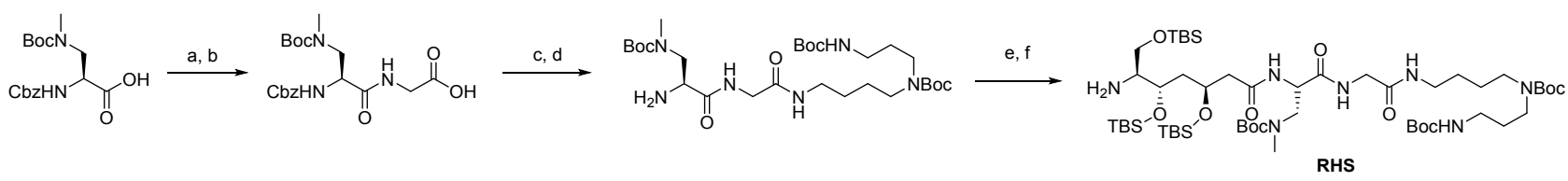

Scheme S7: Synthesis of the right-hand side fragment (RHS):

a) Gly-OMe ${ }^{*} \mathrm{HCl}, \mathrm{HATU}$, DIPEA, DMF, $0{ }^{\circ} \mathrm{C}$ to rt, $24 \mathrm{~h}, 85 \%$; b) $\mathrm{LiOH}^{*} \mathrm{H}_{2} \mathrm{O}, \mathrm{H}_{2} \mathrm{O} / \mathrm{MeOH} / \mathrm{THF}(1: 1: 1), 0{ }^{\circ} \mathrm{C}$ to rt, $2 \mathrm{~h}$;

c) $\mathrm{H}-4,3-\mathrm{Spd}(\mathrm{Boc})_{2}$, TBTU, DIPEA, DMF, $0{ }^{\circ} \mathrm{C}$ to rt, $24 \mathrm{~h}$, (72\% over 2 steps); d) $\mathrm{H}_{2}, \mathrm{Pd} / \mathrm{C}, \mathrm{MeOH}, \mathrm{rt}, 24 \mathrm{~h}, 91 \%$;

e) Gla 41, HATU, DIPEA, DMF, $0{ }^{\circ} \mathrm{C}$ to rt, $24 \mathrm{~h}, 72 \%$; f) $\mathrm{H}_{2}, \mathrm{Pd} / \mathrm{C}, \mathrm{MeOH}$, rt, $24 \mathrm{~h}, 91 \%$.

\section{Cbz-L-mDAP(Boc)-Gly-OH}<smiles>CN(C[C@H](NC(=O)OCc1ccccc1)C(=O)OCc1ccccc1)C(=O)O</smiles>

To a solution of Cbz-mDAP(Boc)-OH 8 ( $1.16 \mathrm{~g}, 3.28 \mathrm{mmol}, 1.00 \mathrm{eq}$.) in $10 \mathrm{~mL}$ of DMF was added HATU $\left(1.37 \mathrm{~g}, 3.61 \mathrm{mmol}, 1.10 \mathrm{eq}\right.$.) at $0{ }^{\circ} \mathrm{C}$. The mixture was stirred for 10 minutes and then a solution of $\mathrm{HCl}$-Gly-OMe (494 mg, $3.94 \mathrm{mmol}, 1.20$ eq.) and DIPEA (1.40 mL, $9.84 \mathrm{mmol}, 3.00$ eq.) in $10 \mathrm{~mL}$ of DMF was added at $0{ }^{\circ} \mathrm{C}$. The mixture was warmed to room temperature and stirred for $24 \mathrm{~h}$. The reaction mixture was then diluted with EtOAc. The organic phase was washed with $1 \mathrm{~N} \mathrm{HCl}(3 x)$, sat. $\mathrm{NaHCO}_{3}$ solution (3x) and brine, dried over $\mathrm{Na}_{2} \mathrm{SO}_{4}$, filtered and the solvent was removed under reduced pressure. The residue was further purified by column chromatography $\left(\mathrm{SiO}_{2}, \mathrm{Hex}\right.$ :EtOAc - 1:1) to give the dipeptide methyl ester $(1.39 \mathrm{~g}, 85 \%)$ as a colorless foam.

To a solution of Cbz-mDAP(Boc)Gly-OMe 31 (522 mg, $1.23 \mathrm{mmol}, 1.00$ eq.) in $5 \mathrm{~mL}$ of $\mathrm{H}_{2} \mathrm{O}, 5 \mathrm{~mL}$ of THF and $5 \mathrm{~mL}$ of $\mathrm{MeOH}$, cooled down to $0{ }^{\circ} \mathrm{C}$, was added $\mathrm{LiOH}^{*} \mathrm{H}_{2} \mathrm{O}$ (104 mg, $2.47 \mathrm{mmol}, 2.00$ eq.). The solution was stirred for $1 \mathrm{~h}$ at $0{ }^{\circ} \mathrm{C}$ and for $3 \mathrm{~h}$ room temperature before it was acidified with $0.5 \mathrm{M}$ $\mathrm{HCl}$. The aqueous phase was extracted with EtOAc three times and dried over $\mathrm{Na}_{2} \mathrm{SO}_{4}$. After filtration the volatiles were removed under reduced pressure. ${ }^{1} \mathrm{H}-\mathrm{NMR}\left(500 \mathrm{MHz}, \mathrm{CDCl}_{3}\right): \delta(\mathrm{ppm})=1.39(\mathrm{~s}, 9$ $\mathrm{H}), 2.86(\mathrm{~s}, 3 \mathrm{H}), 3.37(\mathrm{~d}, \mathrm{~J}=13.8 \mathrm{~Hz}, 1 \mathrm{H}), 3.71-3.80(\mathrm{~m}, 1 \mathrm{H}), 3.82-3.94(\mathrm{~m}, 1 \mathrm{H}), 4.13-4.24(\mathrm{~m}, 1 \mathrm{H})$, 4.56-4.63 (m, $1 \mathrm{H}), 5.01-5.15(\mathrm{~m}, 2 \mathrm{H}), 5.29(\mathrm{br} \mathrm{s}, 1 \mathrm{H})$, 7.28-7.36 (m, $5 \mathrm{H}) .{ }^{13} \mathrm{C}-\mathrm{NMR}\left(126 \mathrm{MHz}, \mathrm{CDCl}_{3}\right)$ : $\delta(\mathrm{ppm})=28.4,35.3,41.5,50.2,65.4,67.2,81.2,127.1,127.8,128.1,128.5,128.6,128.7,156.9$, 157.3, 170.6, 172.3. HRMS (ESI) m/z calc for $\mathrm{C}_{19} \mathrm{H}_{27} \mathrm{~N}_{3} \mathrm{O}_{7}[\mathrm{M}+\mathrm{H}]^{+} 410.1927$ found 410.1932. 


\section{$\mathrm{NH}_{2}$-L-mDAP(Boc)-Gly-4,3-Spd(Boc) ${ }_{2}$}<smiles>CN(C[C@H](N)C(=O)OCc1ccccc1)C(=O)NCCCNC(=O)[O-]</smiles>

The above acid was dissolved in $5 \mathrm{~mL}$ of DMF and DIPEA ( $430 \mu \mathrm{L}, 2.47 \mathrm{mmol}, 2.00$ eq.) and TBTU (435mg, $1.36 \mathrm{mmol}, 1.10$ eq.) were added at $0{ }^{\circ} \mathrm{C}$. The mixture was stirred for 10 minutes and then a solution of $\mathrm{H}-\mathrm{Spd}(\mathrm{Boc}))_{2}\left(468 \mathrm{mg}, 1.36 \mathrm{mmol}, 1.10 \mathrm{eq}\right.$.) in $5 \mathrm{~mL}$ of DMF was added at $0{ }^{\circ} \mathrm{C}$. The mixture was warmed to room temperature and stirred for $24 \mathrm{~h}$. The reaction mixture was then diluted with EtOAc. The organic phase was washed with $1 \mathrm{~N} \mathrm{HCl}(2 \mathrm{x})$, sat. $\mathrm{NaHCO}_{3}$ solution $(2 \mathrm{x})$ and brine, dried over $\mathrm{Na}_{2} \mathrm{SO}_{4}$, filtered and the solvent was removed under reduced pressure. The residue was further purified by column chromatography $\left(\mathrm{SiO}_{2}, 98.5: 1.5\right.$ to $\left.96.5: 3.5-\mathrm{DCM} / \mathrm{MeOH}\right)$ to give the pseudotripeptide 32 (650 $\mathrm{mg}, 72 \%)$ as a colorless solid.

Pseudotripeptide 32 (503 mg, $0.683 \mathrm{mmol}, 1.00$ eq.) was dissolved in $4 \mathrm{~mL}$ of $\mathrm{MeOH}$ and $\mathrm{Pd} / \mathrm{C}$ (10 $\mathrm{wt} \%, 51 \mathrm{mg}$ ) was added. The reaction was stirred for $24 \mathrm{~h}$ at room temperature under an atmosphere of hydrogen. The mixture was filtered through a pad of Celite and the volatiles were removed under reduced pressure. The crude product was purified with column chromatography $\left(\mathrm{SiO}_{2}, 97: 3\right.$ to 9:1 $\mathrm{DCM} / \mathrm{MeOH})$ to yield the free amine $(374 \mathrm{mg}, 91 \%)$ as a colorless solid. ${ }^{1} \mathrm{H}-\mathrm{NMR}\left(500 \mathrm{MHz}, \mathrm{CDCl}_{3}\right): \delta$ $(\mathrm{ppm})=1.41(\mathrm{~s}, 9 \mathrm{H}), 1.43(\mathrm{~s}, 9 \mathrm{H}), 1.45-1.55(\mathrm{~m}, 4 \mathrm{H}), 1.59-1.67$ (br s, $2 \mathrm{H}), 2.53-2.65(\mathrm{br} \mathrm{s}, 2 \mathrm{H}), 2.86$ (s, $3 \mathrm{H}), 3.04-3.09(\mathrm{~m}, 2 \mathrm{H}), 3.10-3.17(\mathrm{~m}, 2 \mathrm{H}), 3.17-3.28(\mathrm{~m}, 4 \mathrm{H}), 3.49-3.54(\mathrm{~m}, 1 \mathrm{H}), 3.57-3.64(\mathrm{~m}, 1$ $\mathrm{H})$, 3.84-3.93 (m, $1 \mathrm{H}), 8.08-8.34(\mathrm{br} \mathrm{s}, 1 \mathrm{H})$. HRMS (ESI) $\mathrm{m} / \mathrm{z}$ calc for $\mathrm{C}_{28} \mathrm{H}_{54} \mathrm{~N}_{6} \mathrm{O}_{8}[\mathrm{M}+\mathrm{H}]^{+} 603.4076$ found 603.4065 .

\section{Cbz-(3S,5S,6S)-Gla(7-TBDMS, 3,5-0,0-TBMS)-L-mDAP(Boc)-Gly-4,3-Spd(Boc) ${ }_{2}$ (RHS)}

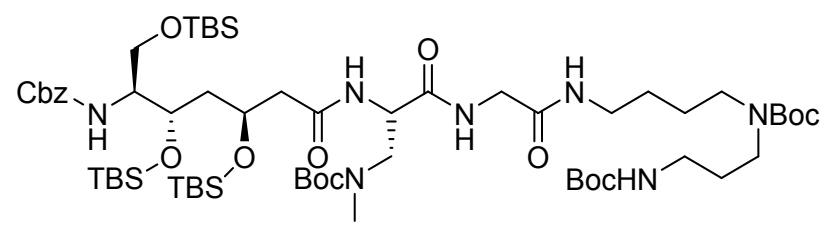

Galantinic acid derivative 40 (317 mg, $0.601 \mathrm{mmol}, 1.00$ eq.) was dissolved in $6 \mathrm{~mL}$ of DMF and cooled to $0{ }^{\circ} \mathrm{C}$. To this solution HATU (251 $\mathrm{mg}, 0.660 \mathrm{mmol}, 1.10 \mathrm{eq}$.) was added. After 5 minutes a solution of $\mathrm{H}-m \mathrm{DAP}(\mathrm{Boc}) \mathrm{GlySpd}(\mathrm{Boc})$ (398 $_{\mathrm{mg}} 0.661 \mathrm{mmol}, 1.10 \mathrm{eq}$ ) and DIPEA (112 $\mu \mathrm{L}, 0.660 \mathrm{mmol}, 1.10$ eq.) in $3 \mathrm{~mL}$ of DMF was added at $0{ }^{\circ} \mathrm{C}$. The mixture was warmed to room temperature and stirred for $24 \mathrm{~h}$. The reaction mixture was then diluted with EtOAc. The organic phase was washed with $0.5 \mathrm{~N} \mathrm{HCl}$ (2x), $\mathrm{NaHCO}_{3}(2 \mathrm{x})$ and brine, dried over $\mathrm{Na}_{2} \mathrm{SO}_{4}$, filtered and the solvent was removed under reduced pressure. The residue was further purified by column chromatography $\left(\mathrm{SiO}_{2}, 98: 2-\mathrm{DCM} / \mathrm{MeOH}\right)$ to give the right-hand side of the paenilamicins ( $455 \mathrm{mg}, 72 \%)$ RHS as a colorless wax. ${ }^{1} \mathbf{H}$-NMR (400 $\left.\mathrm{MHz}, \mathrm{CDCl}_{3}\right): \delta(\mathrm{ppm})=0.04(\mathrm{~s}, 6 \mathrm{H}), 0.06(\mathrm{~s}, 6 \mathrm{H}), 0.07(\mathrm{~s}, 6 \mathrm{H}), 0.85(\mathrm{~s} .9 \mathrm{H}), 0.86(\mathrm{~s}, 9 \mathrm{H}), 0.87$ (s, 9 
$\mathrm{H}), 1.42(\mathrm{~s}, 9 \mathrm{H}), 1.44(\mathrm{~s}, 9 \mathrm{H}), 1.44(\mathrm{~s}, 9 \mathrm{H}), 1.47-1.56(\mathrm{~m}, 4 \mathrm{H}), 1.58-1.68(\mathrm{~m}, 2 \mathrm{H}), 1.79-1.89(\mathrm{~m}, 1 \mathrm{H})$, $2.26(\mathrm{dd}, \mathrm{J}=14.8,6.0 \mathrm{~Hz}, 1 \mathrm{H}), 2.37-2.52(\mathrm{~m}, 1 \mathrm{H}), 2.89(\mathrm{~s}, 3 \mathrm{H}), 3.02-3.33(\mathrm{~m}, 8 \mathrm{H}), 3.41-3.87(\mathrm{~m}, 6$ $\mathrm{H})$, 3.94-4.15 (m, 3 H), 4.41 (br s, $1 \mathrm{H}), 4.96-5.08(\mathrm{~m}, 2 \mathrm{H}), 6.96$ (br s, $1 \mathrm{H}), 7.13$ (br s, $1 \mathrm{H}), 7.27-7.37$ $(\mathrm{m}, 5 \mathrm{H}) .{ }^{13} \mathrm{C}-\mathrm{NMR}\left(100 \mathrm{MHz}, \mathrm{CDCl}_{3}\right): \delta(\mathrm{ppm})=-5.3,-5.2,-4.8,-4.7,-4.5,-4.0,18.0,18.1,18.2,26.0$, 26.0, 26.0, 28.4, 28.6, 38.7, 43.5, 43.5, 50.7, 54.7, 61.6, 65.4, 67.0, 80.7, 127.1, 127.7, 128.3, 128.5, 128.7, 128.7, 136.5, 156.3, 168.8, 170.4. NMR spectra contain signals of DMF. HRMS (ESI) m/z calc for $\mathrm{C}_{61} \mathrm{H}_{115} \mathrm{~N}_{7} \mathrm{O}_{14} \mathrm{Si}_{3}[\mathrm{M}+\mathrm{H}]^{+} 1254.7888$ found 1254.7896 .

Cbz-Gla-mDAP(Boc)-Gly-(4,3)-Spd (292 mg, $0.262 \mathrm{mmol}, 1.00$ eq.) was dissolved in $9 \mathrm{~mL}$ of $\mathrm{MeOH}$ and $\mathrm{Pd} / \mathrm{C}(10 \mathrm{wt} \%, 29 \mathrm{mg})$ was added. The reaction was stirred for $24 \mathrm{~h}$ at room temperature under an atmosphere of hydrogen. The mixture was filtered through a pad of Celite and the volatiles were removed in vacuo. The crude product was purified with column chromatography $\left(\mathrm{SiO}_{2}, 96.5: 3.5-\right.$ $\mathrm{DCM} / \mathrm{MeOH})$ to yield the free amine $(233 \mathrm{mg}, 91 \%)$ as a colorless solid.

HRMS (ESI) m/z calc for $\mathrm{C}_{53} \mathrm{H}_{110} \mathrm{~N}_{7} \mathrm{O}_{12} \mathrm{Si}_{3}[\mathrm{M}+\mathrm{H}]^{+} 1120.7520$ found 1120.7525 . 


\subsection{Synthesis of PamB2 - Condensation of LHS and RHS fragments and overall deprotection}

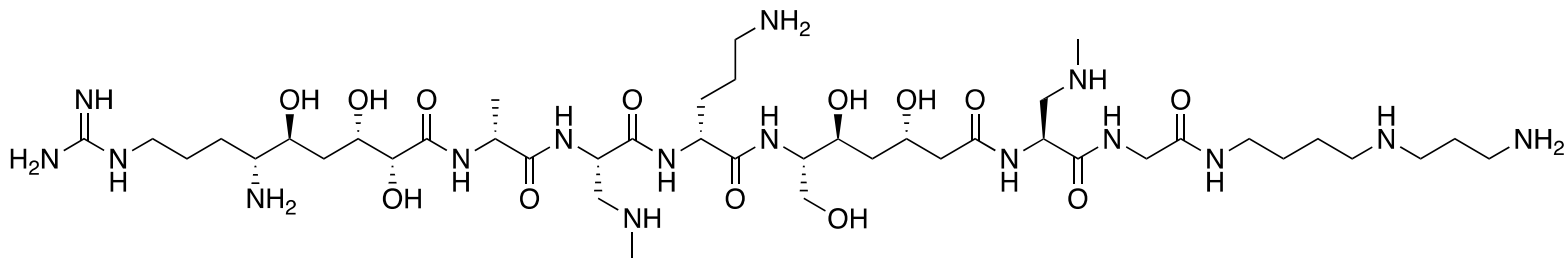

Boc $_{3}$-Agm-Hpa(diacetonid)-D-Ala-L-mDAP(Boc)-D-Orn(Boc)-OH (LHS) $(47.4 \mathrm{mg}, 0.0415 \mathrm{mmol}, 1.30$ eq.) and (3S,5R,6S)-H-Gla(7-OTBS, 3,5-O,O-TBS)-mDAP(Boc)-Gly-Spd(Boc) $)_{2}$ (RHS) $(32.2$ mg, $0.0345 \mathrm{mmol}, 1.00$ eq.) were dissolved in $2 \mathrm{~mL}$ of DCM and DIPEA (7.05 $\mu \mathrm{L}, 0.0415 \mathrm{mmol}, 1.40$ eq.) was added at $0{ }^{\circ} \mathrm{C}$. Next EDC. $\mathrm{HCl}(7.95 \mathrm{mg}, 0.0415 \mathrm{mmol}, 1.20$ eq.) and $\mathrm{HOBt}(6.53 \mathrm{mg}, 0.0484 \mathrm{mmol}$, 1.40 eq.) were added together in one portion. The mixture was warmed to room temperature and stirred for $48 \mathrm{~h}$. The reaction mixture was then diluted with EtOAc. The organic phase was washed with $1 \mathrm{~N}$ $\mathrm{HCl}(2 \mathrm{c})$, sat. $\mathrm{NaHCO}_{3}$ solution (2x) and brine, dried over $\mathrm{Na}_{2} \mathrm{SO}_{4}$, filtered and the solvent was removed under reduced pressure. The residue was further purified by column chromatography $\left(\mathrm{SiO}_{2}, 98: 2\right.$ to $97: 3-\mathrm{DCM} / \mathrm{MeOH})$ to give the fully protected paenilamicin $\mathrm{B} 2(44.8 \mathrm{mg}, 63 \%)$ as a colorless solid after lyophilization.

HRMS (ESI) $\mathrm{m} / \mathrm{z}$ calc for $\mathrm{C}_{101} \mathrm{H}_{192} \mathrm{~N}_{16} \mathrm{O}_{27} \mathrm{Si}_{3}[\mathrm{M}+\mathrm{H}]^{+} 2147.3562$ found 2147.4009 (direct injection).

Fully protected paenilamicin B2 (50.7 mg, $0.0246 \mathrm{mmol}, 1.00$ eq.) was dissolved in $2 \mathrm{~mL}$ of DCM and $2 \mathrm{~mL}$ of TFA and was stirred for $5 \mathrm{~h}$ at room temperature. The volatiles were removed under reduced pressure and the residue was precipitated with cold $\mathrm{Et}_{2} \mathrm{O}$. The solid was filtered and washed with cold $\mathrm{Et}_{2} \mathrm{O}$ before it was dissolved in $\mathrm{H}_{2} \mathrm{O}$ and lyophilized. The residue was taken up in water and further purified using an PLRP-S column (solvent A: water $+0.1 \%$ TFA, solvent B: acetonitrile $+0.1 \%$ TFA, flow rate $=50 \mathrm{~mL} / \mathrm{min}$, gradient: $1 \% \mathrm{~B}$ for $20 \mathrm{~min}, 1$ to $100 \% \mathrm{~B}$ in $5 \mathrm{~min}$, hold at $100 \% \mathrm{~B}$ for $10 \mathrm{~min}$ ). Paenilamicin B2 containing fractions were combined and lyophilized to give the title compound (14.3 $\mathrm{mg}, 57 \%)$ as a colorless solid.

HRMS (ESI) $\mathrm{m} / \mathrm{z}$ calc for $\mathrm{C}_{42} \mathrm{H}_{86} \mathrm{~N}_{16} \mathrm{O}_{13}[\mathrm{M}+\mathrm{H}]^{+} 1023.6639$ found 1023.6638 .

For NMR spectroscopy data see Table S1, Figures S1 and S2. 
4. NMR Spectra of the synthesized compounds 
(4R)-N,N -Boc-Cbz-ethyl-4,8-diamino-3-oxooctanoate (42)<smiles>CCOC(=O)CC(=O)[C@H](CCCNC(=O)OCc1ccccc1)NC(=O)OCc1ccccc1</smiles>

${ }^{1} \mathrm{H}-\mathrm{NMR}\left(400 \mathrm{MHz}, \mathrm{CDCl}_{3}\right)$

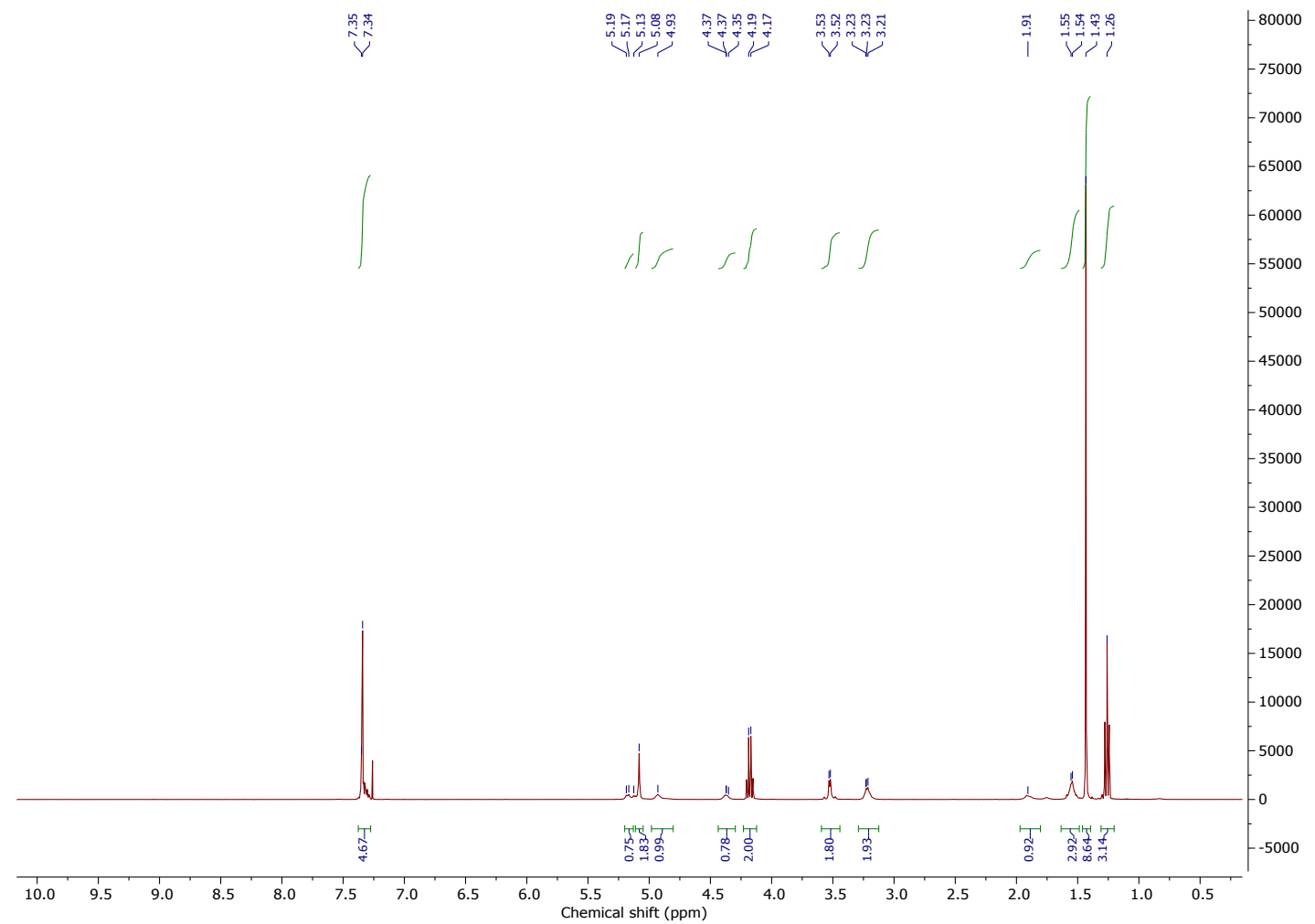

${ }^{13} \mathrm{C}-\mathrm{NMR}\left(100 \mathrm{MHz}, \mathrm{CDCl}_{3}\right)$

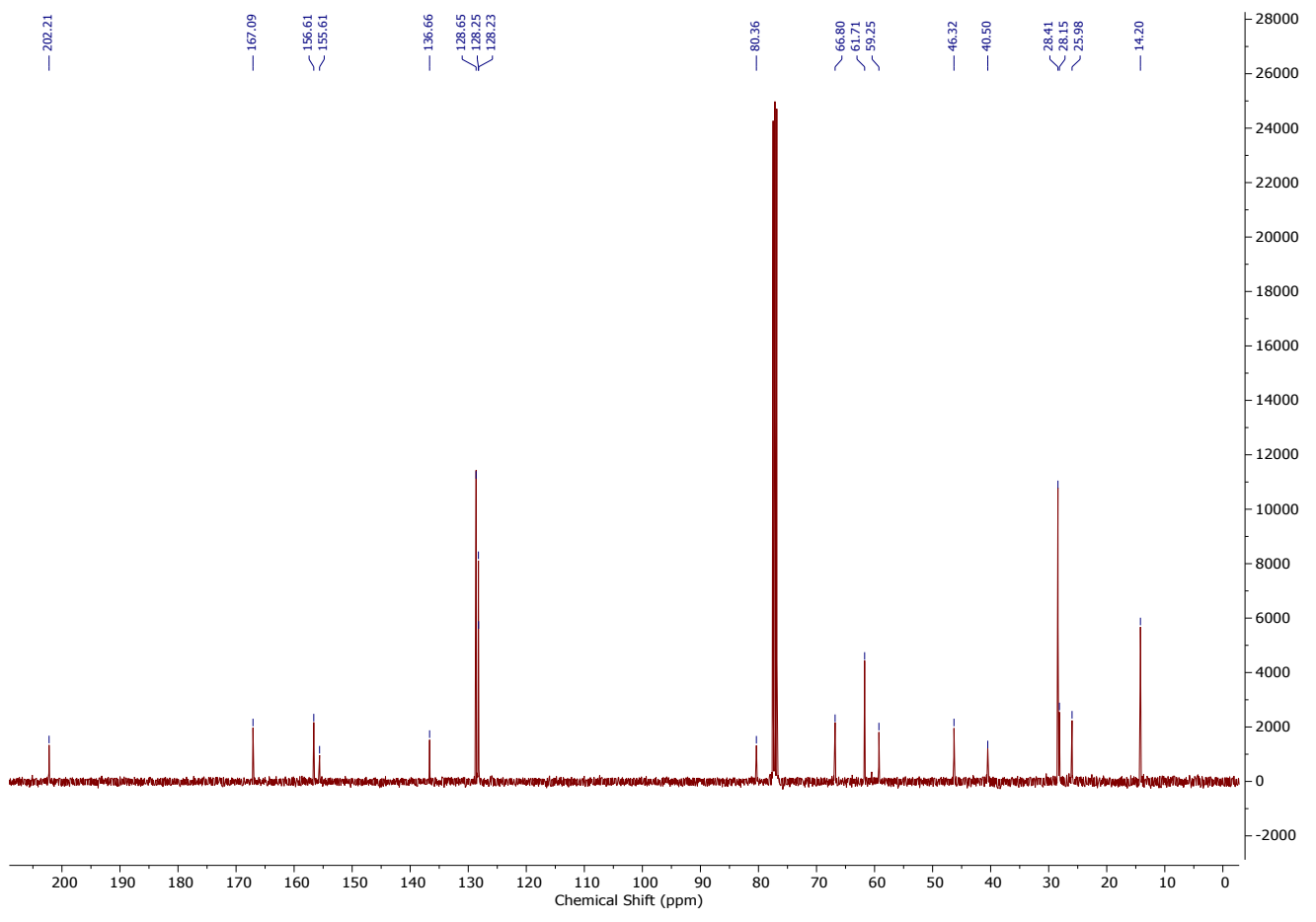


(4R)-N,N -Boc-Cbz-ethyl-4,8-diamino-(3S)-hydroxyoctanoate (43)<smiles>CCOC(=O)C[C@@H](O)[C@H](CCCNC(=O)OCc1ccccc1)NC(=O)OCc1ccccc1</smiles>

${ }^{13} \mathrm{C}-\mathrm{NMR}\left(126 \mathrm{MHz}, \mathrm{CDCl}_{3}\right)$

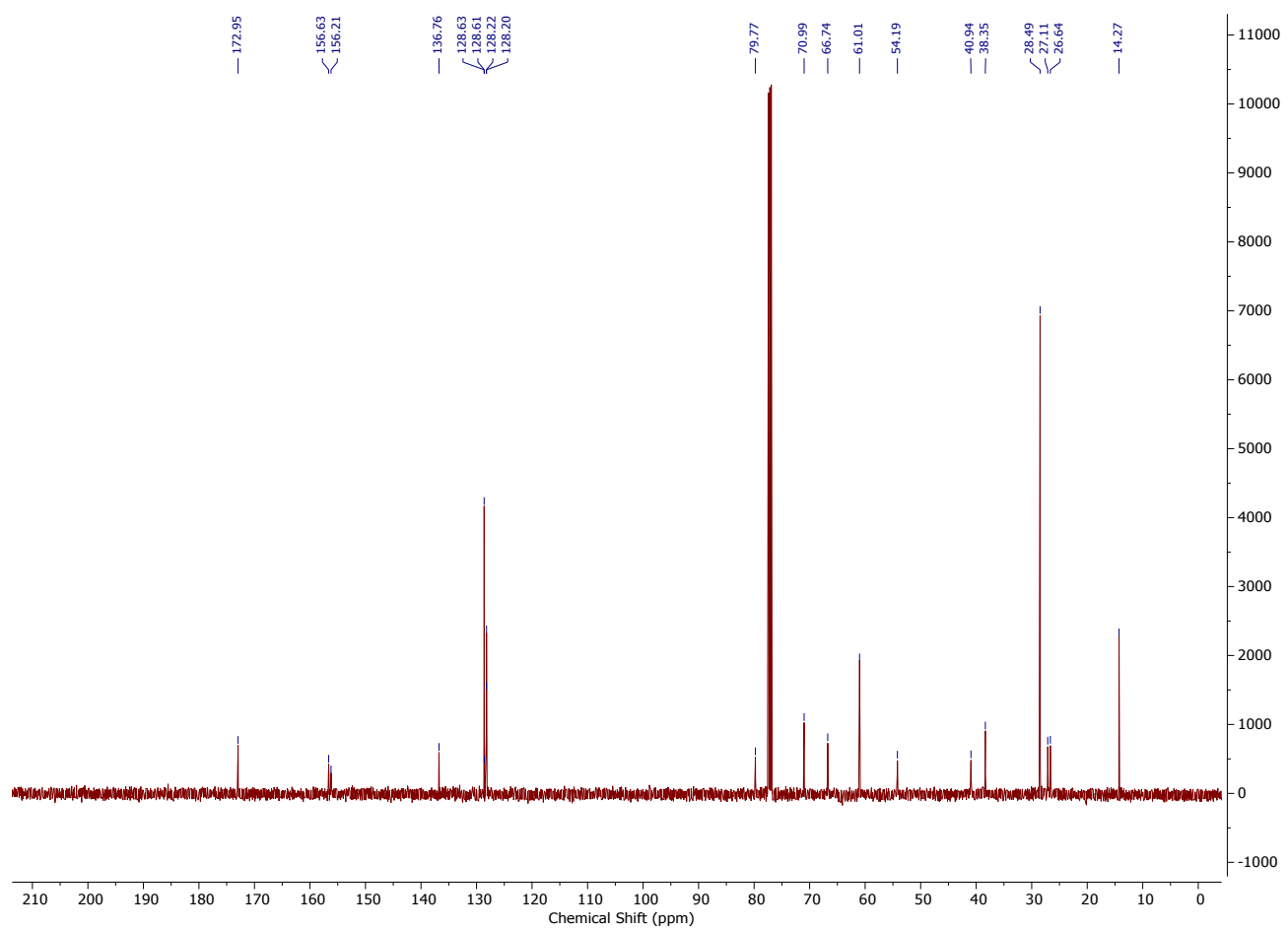

${ }^{1} \mathrm{H}-\mathrm{NMR}\left(500 \mathrm{MHz}, \mathrm{CDCl}_{3}\right)$

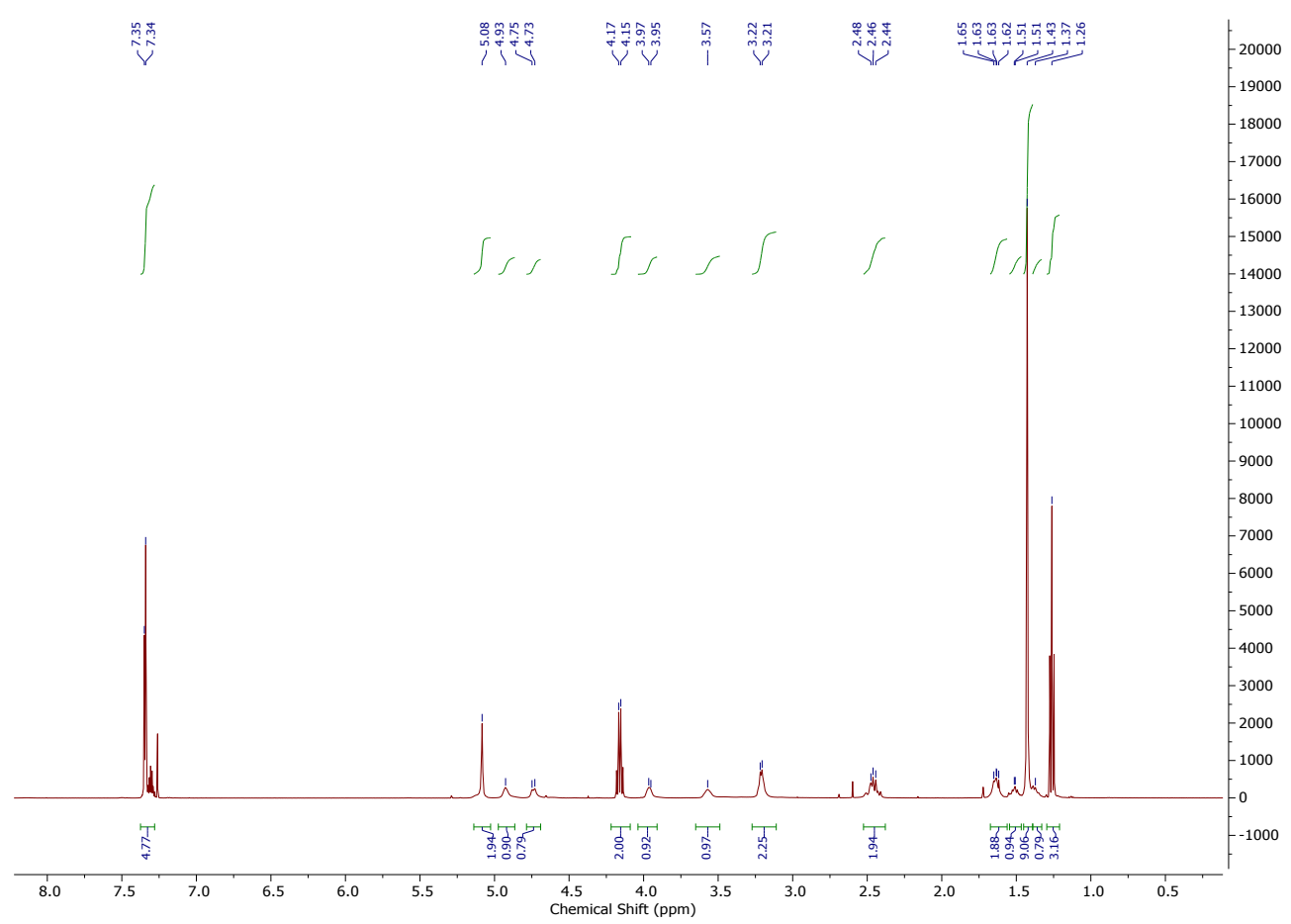


(4R)-N,N -Boc-Cbz-ethyl-4,8-diamino-(3S)-oxy-N,O-acetonidooctanoate (44)<smiles>CCOC(=O)C[C@@H]1OC(C)(C)O[C@H]1CCCNC(=O)OCc1ccccc1</smiles>

${ }^{1} \mathrm{H}-\mathrm{NMR}\left(500 \mathrm{MHz}, \mathrm{CDCl}_{3}\right)$

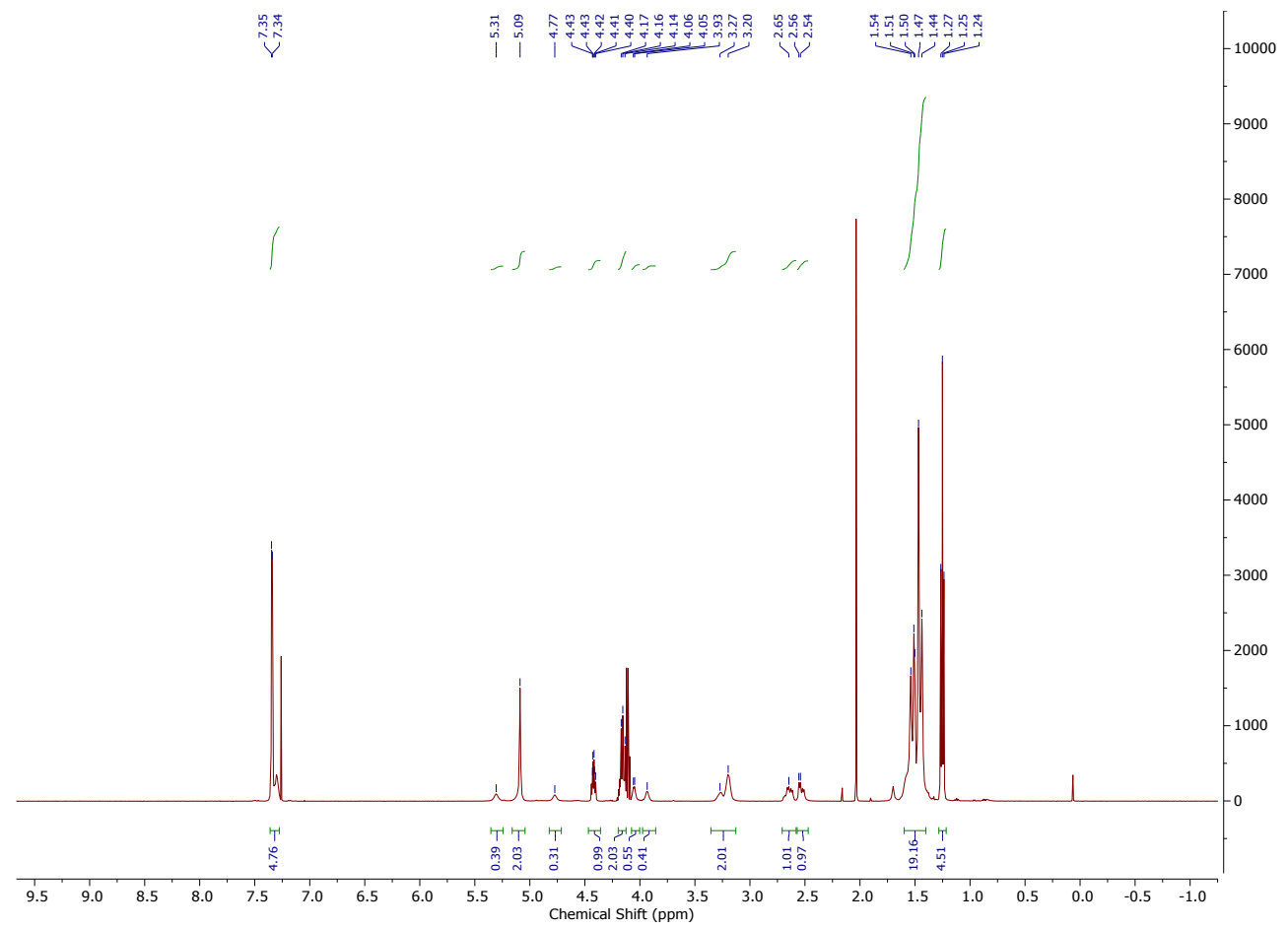

${ }^{13} \mathrm{C}-\mathrm{NMR}\left(126 \mathrm{MHz}, \mathrm{CDCl}_{3}\right)$

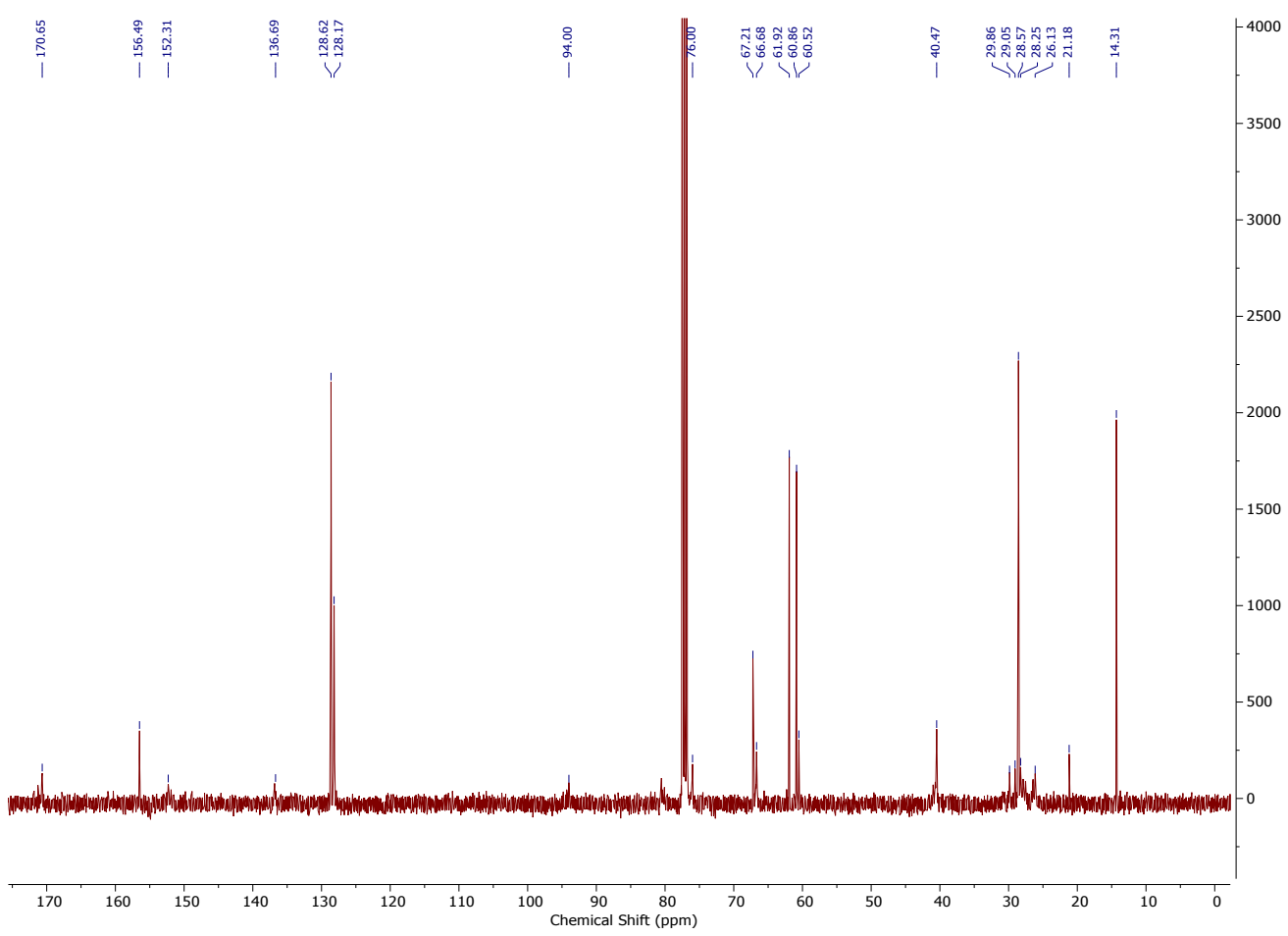


(4R)-N,N -Boc-Arg(Boc ${ }_{2}$ )-ethyl-4,8-diamino-(3S)-oxy-N,O-acetonidooctanoate (45)

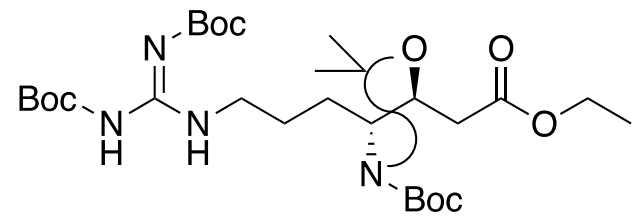

${ }^{1} \mathrm{H}-\mathrm{NMR}\left(500 \mathrm{MHz}, \mathrm{DMSO}-\mathrm{d}_{6}\right)$

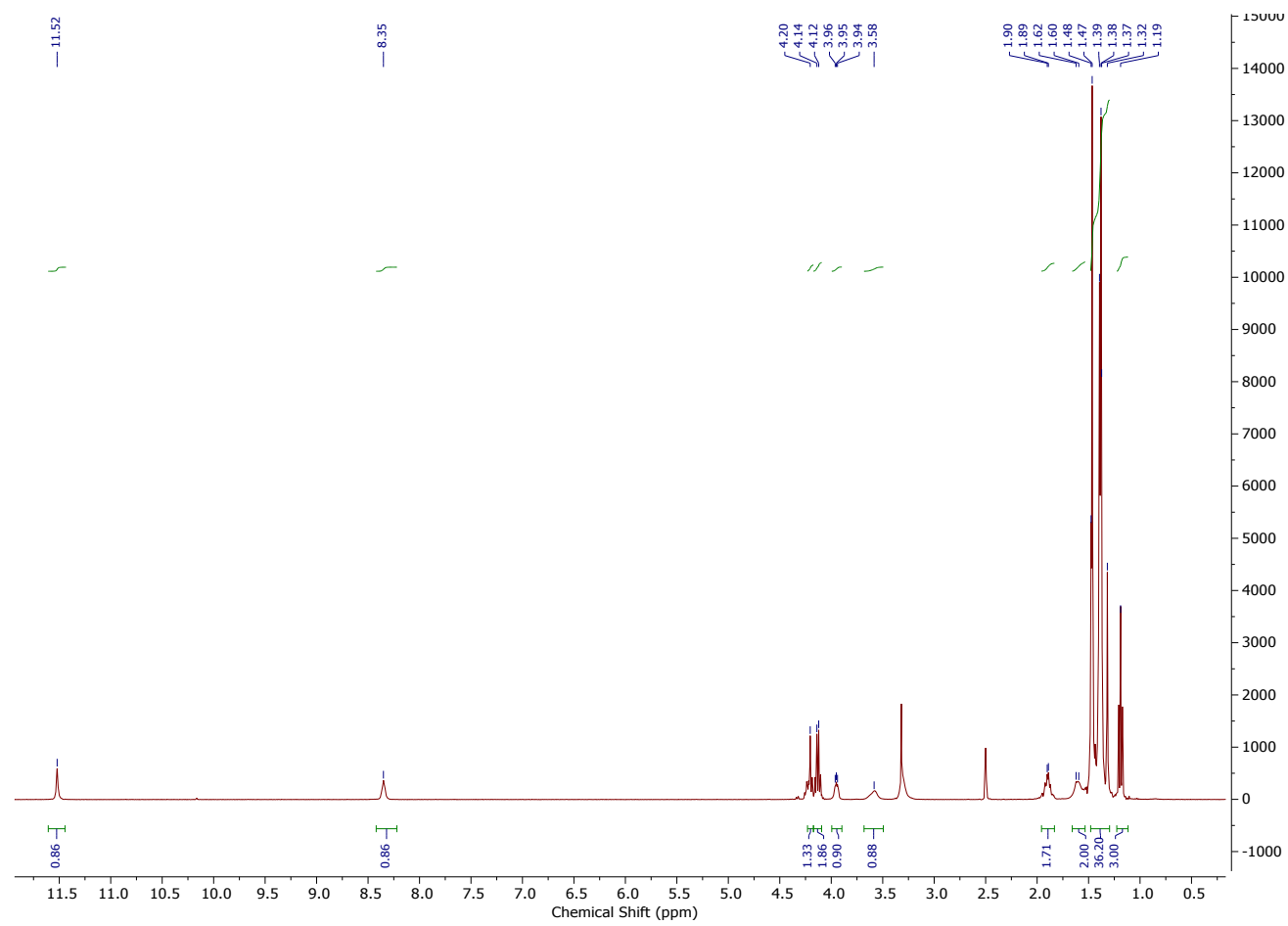

${ }^{13} \mathrm{C}-\mathrm{NMR}$ (126 MHz, DMSO-d 6 )

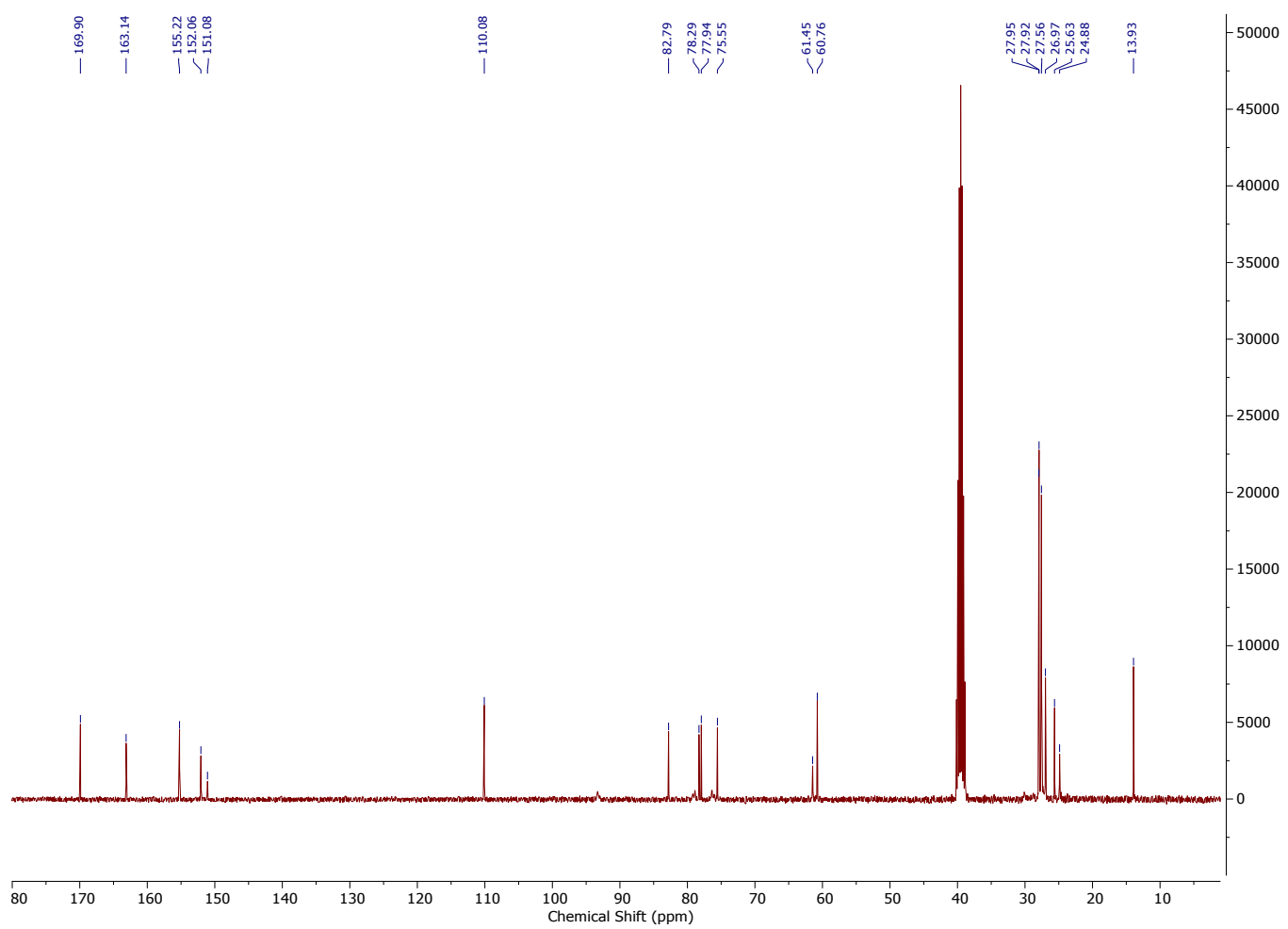


(2E,5S,6R)-N1,N10,N11-tri-Boc-methyl-6,9-diamino-5-hydroxy-6,7-N,Odimethyloxazolidine-non-2- enoate (46)<smiles>COC(=O)/C=C/CC1OC(C)(C)C(CCCNC(=N[R6](=O)OCc2ccccc2)NC(=O)OC(C)(C)C)C1C(=O)OCc1ccccc1</smiles>

${ }^{1} \mathrm{H}-\mathrm{NMR}\left(500 \mathrm{MHz}, \mathrm{CDCl}_{3}\right)$

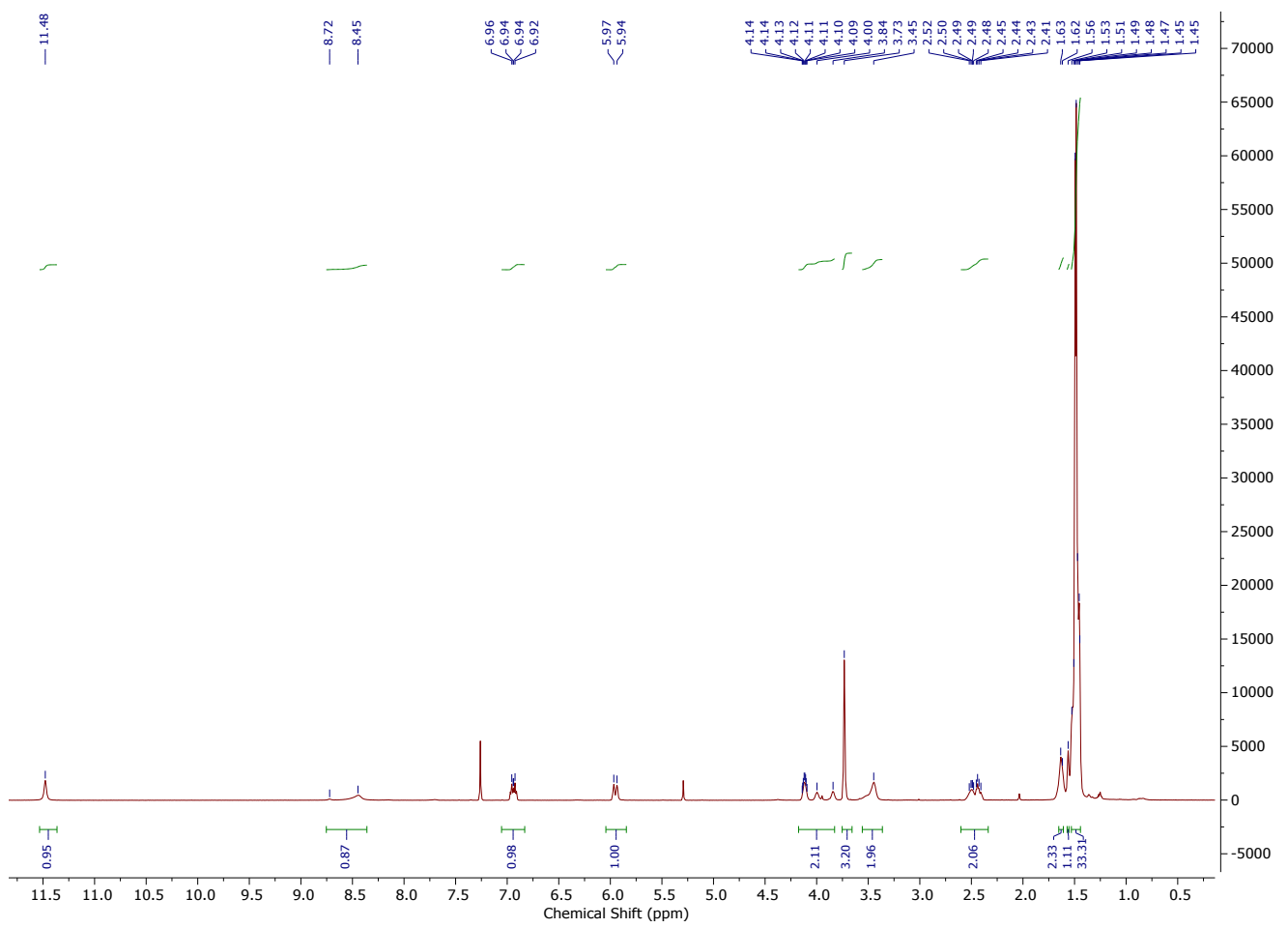

${ }^{13} \mathrm{C}-\mathrm{NMR}\left(126 \mathrm{MHz}, \mathrm{CDCl}_{3}\right)$

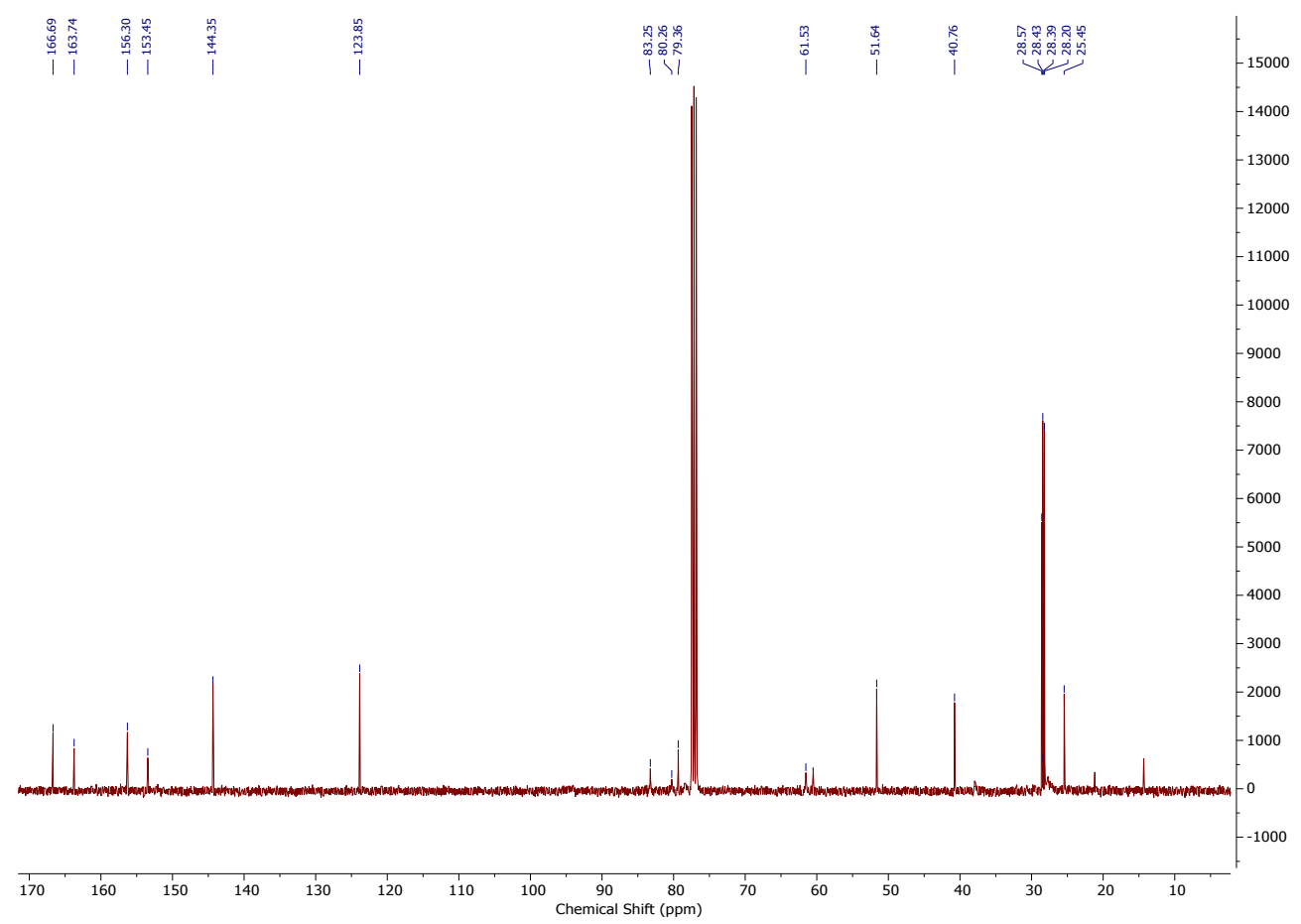


(2R,3S,5S,6R)-N1,N10,N11-tri-Boc-methyl-6-amino-9-guanidino-2,3,5-trihydroxy-6,7$\mathrm{N}, \mathrm{O}$ - dimethyloxazolidine-nonanoate (47)

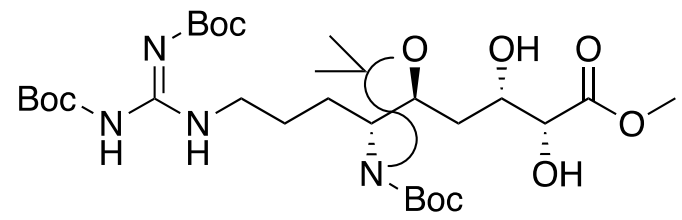

${ }^{1} \mathrm{H}-\mathrm{NMR}\left(500 \mathrm{MHz}, \mathrm{CDCl}_{3}\right)$

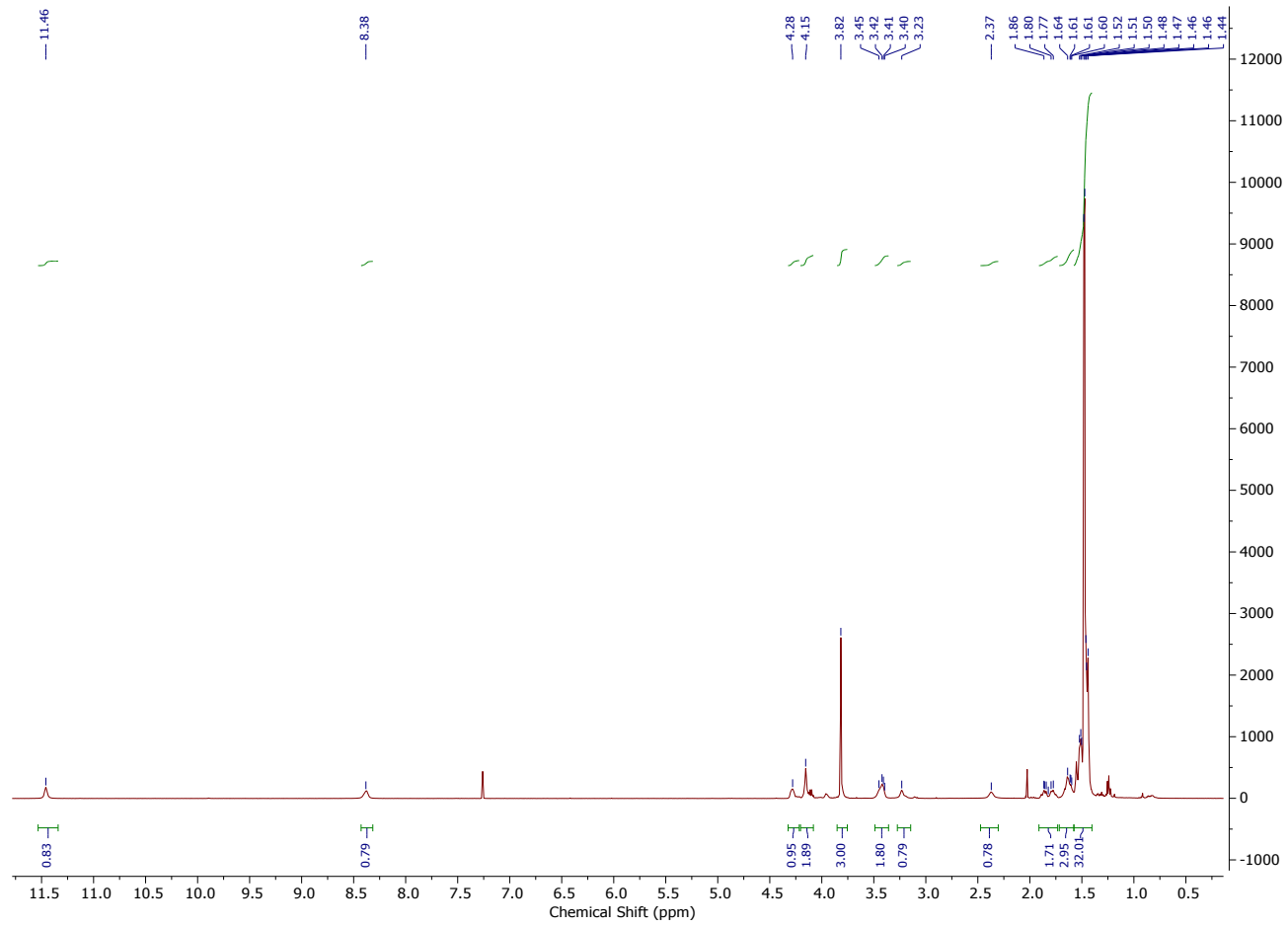

${ }^{13} \mathrm{C}-\mathrm{NMR}\left(126 \mathrm{MHz}, \mathrm{CDCl}_{3}\right)$

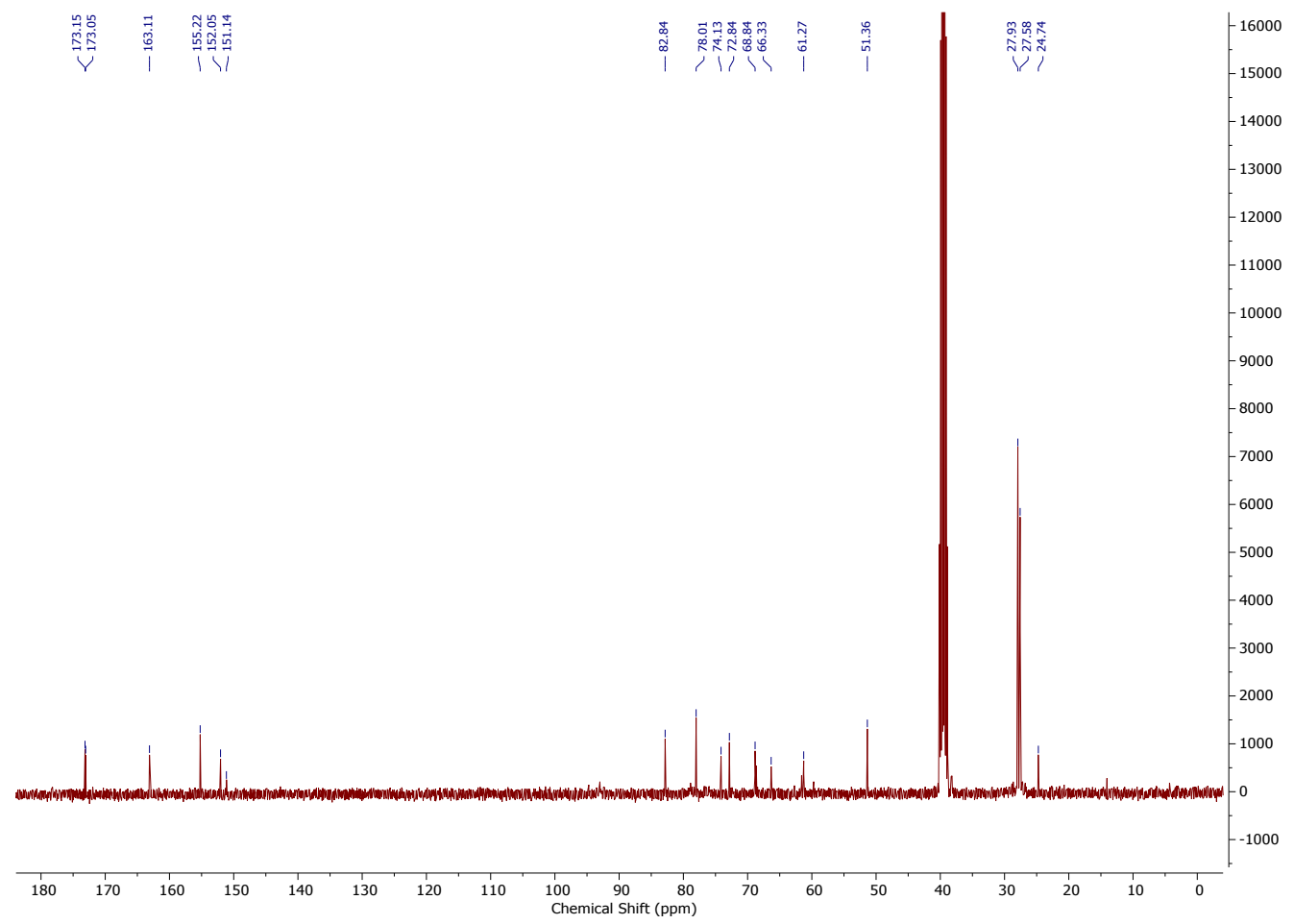


$(2 R, 3 R, 5 S, 6 R)-N 1, N 10, N 11$-tri-Boc-methyl-6-amino-9-guanidino-2,3,5-trihydroxy-6,7$\mathrm{N}, \mathrm{O}$ - dimethyloxazolidine-2,3-O,O-dimethyloxazolidine nonanoate (48)

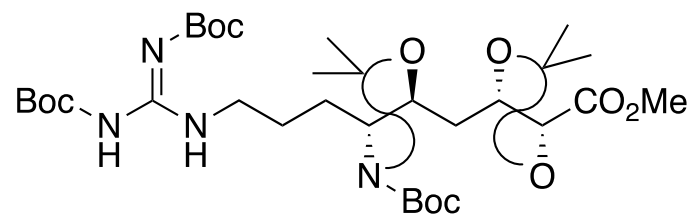

${ }^{1} \mathrm{H}-\mathrm{NMR}\left(400 \mathrm{MHz}, \mathrm{CDCl}_{3}\right)$

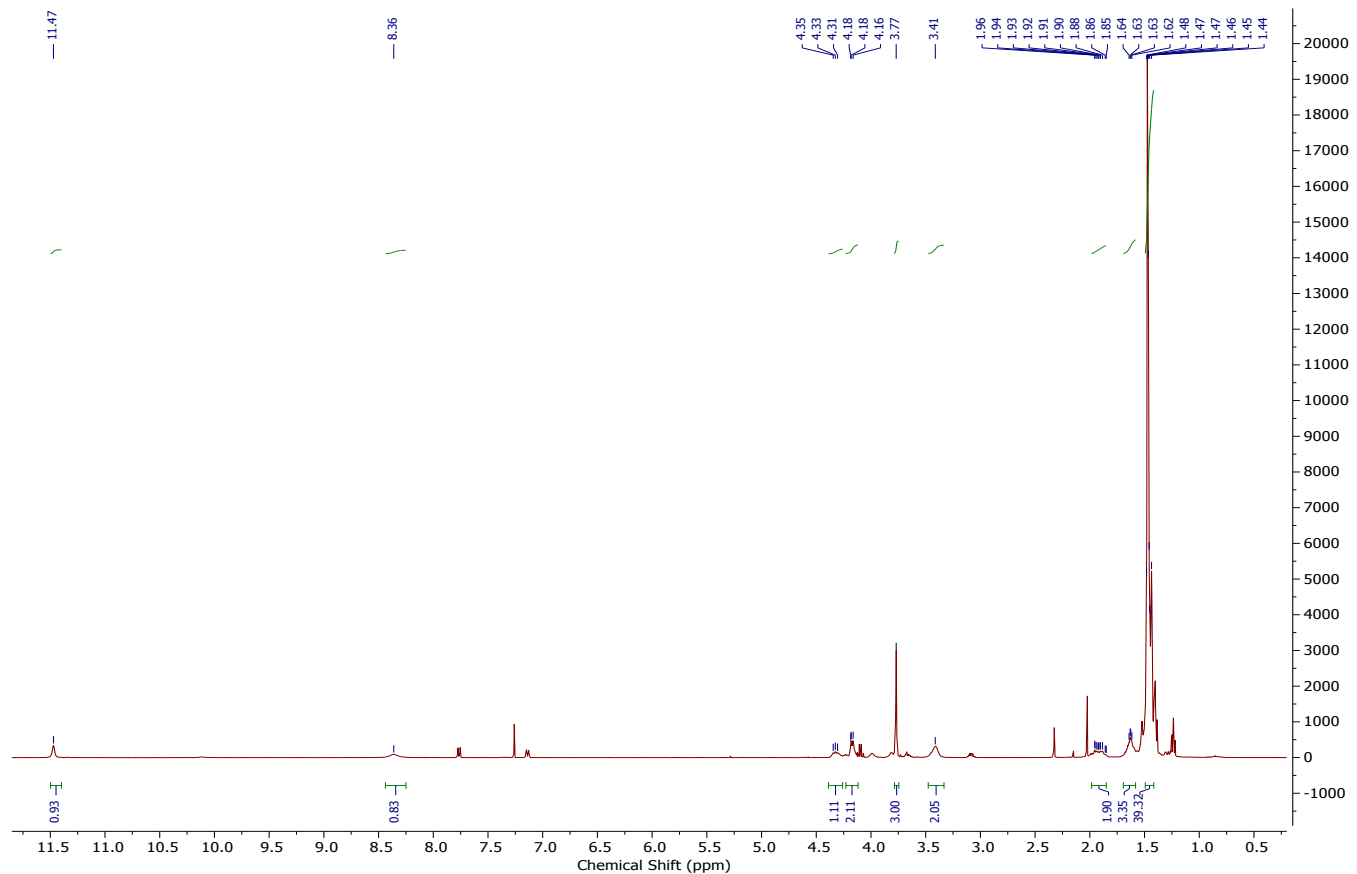

${ }^{13} \mathrm{C}-\mathrm{NMR}\left(100 \mathrm{MHz}, \mathrm{CDCl}_{3}\right)$

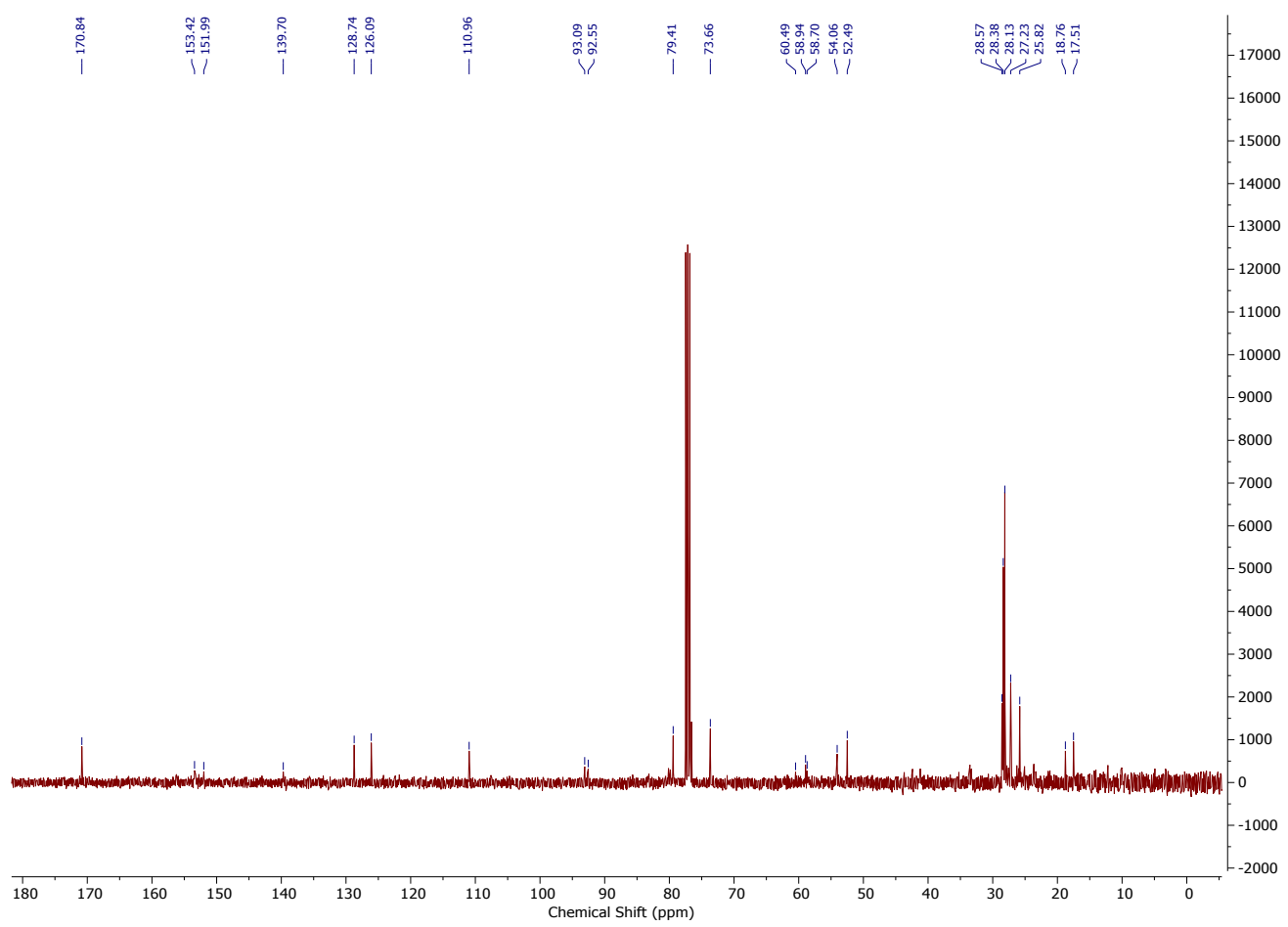


(2R,3S,5S,6R)-N1,N10,N11-tri-Boc-6-amino-9-guanidino-2,3,5-trihydroxy-6,7-N,Odimethyloxazolidine-2,3-0,0-dimethyloxazolidine nonanoic acid (49)

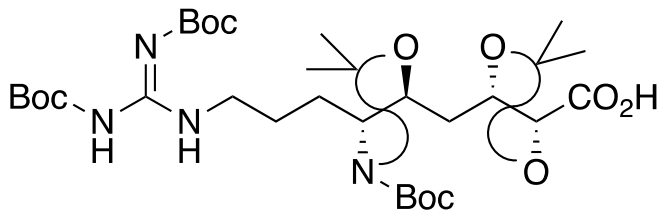

${ }^{1} \mathrm{H}-\mathrm{NMR}\left(500 \mathrm{MHz}, \mathrm{CDCl}_{3}\right)$

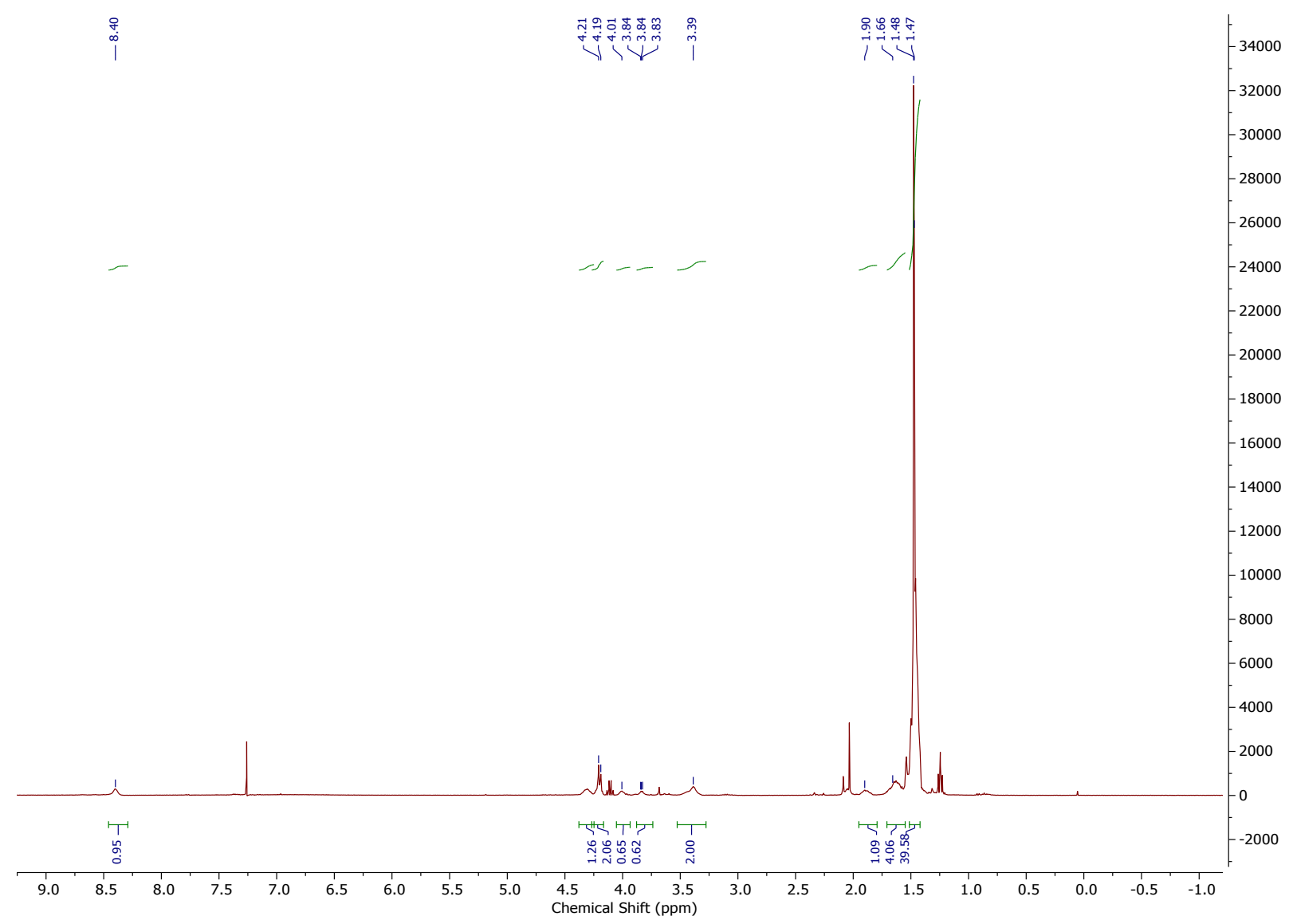


Cbz-L-Ser(TBDMS)-CHO (36)

TBS、<smiles>O=CC(CO)NC(=O)O</smiles>

${ }^{1} \mathrm{H}-\mathrm{NMR}\left(500 \mathrm{MHz}, \mathrm{CDCl}_{3}\right)$

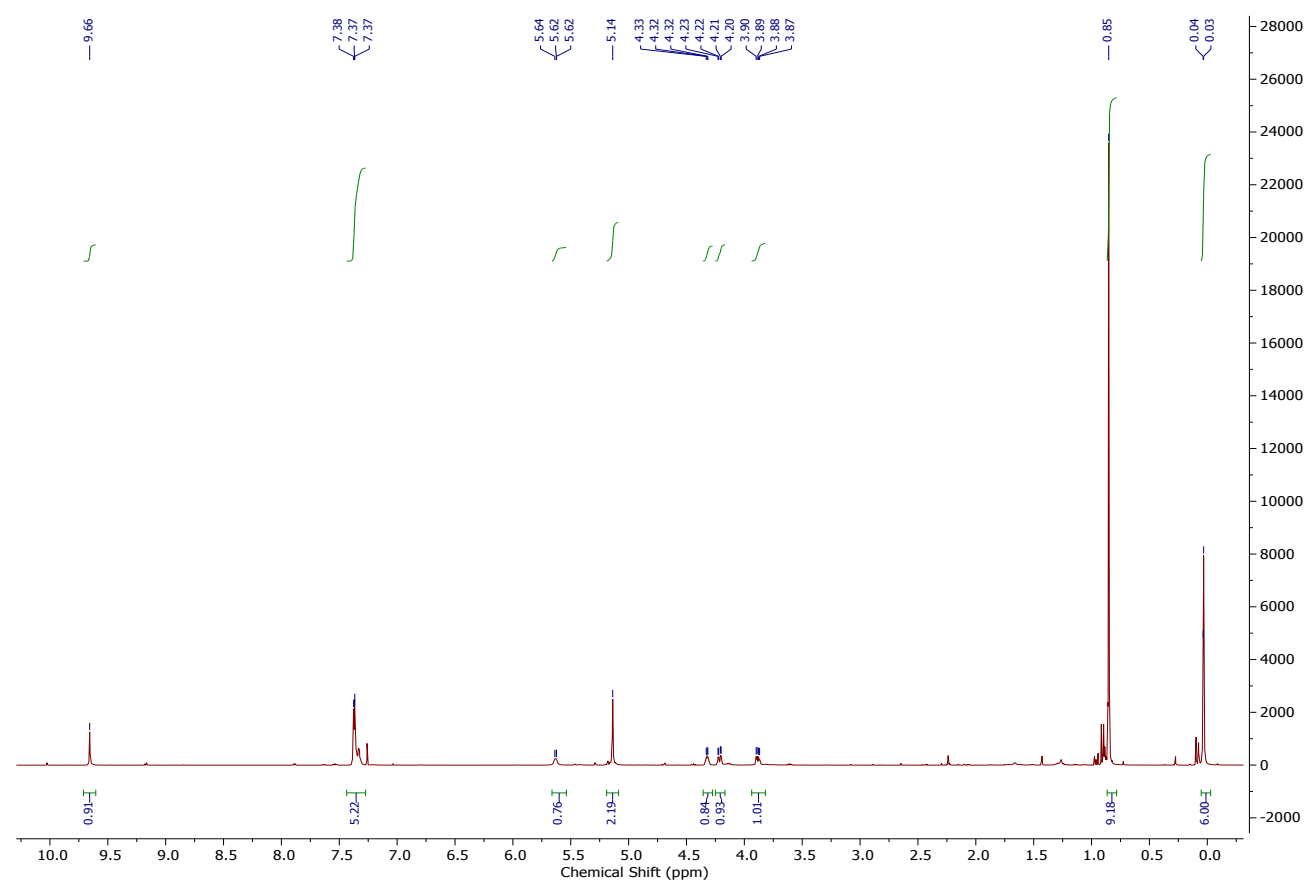

${ }^{13} \mathrm{C}-\mathrm{NMR}\left(126 \mathrm{MHz}, \mathrm{CDCl}_{3}\right)$

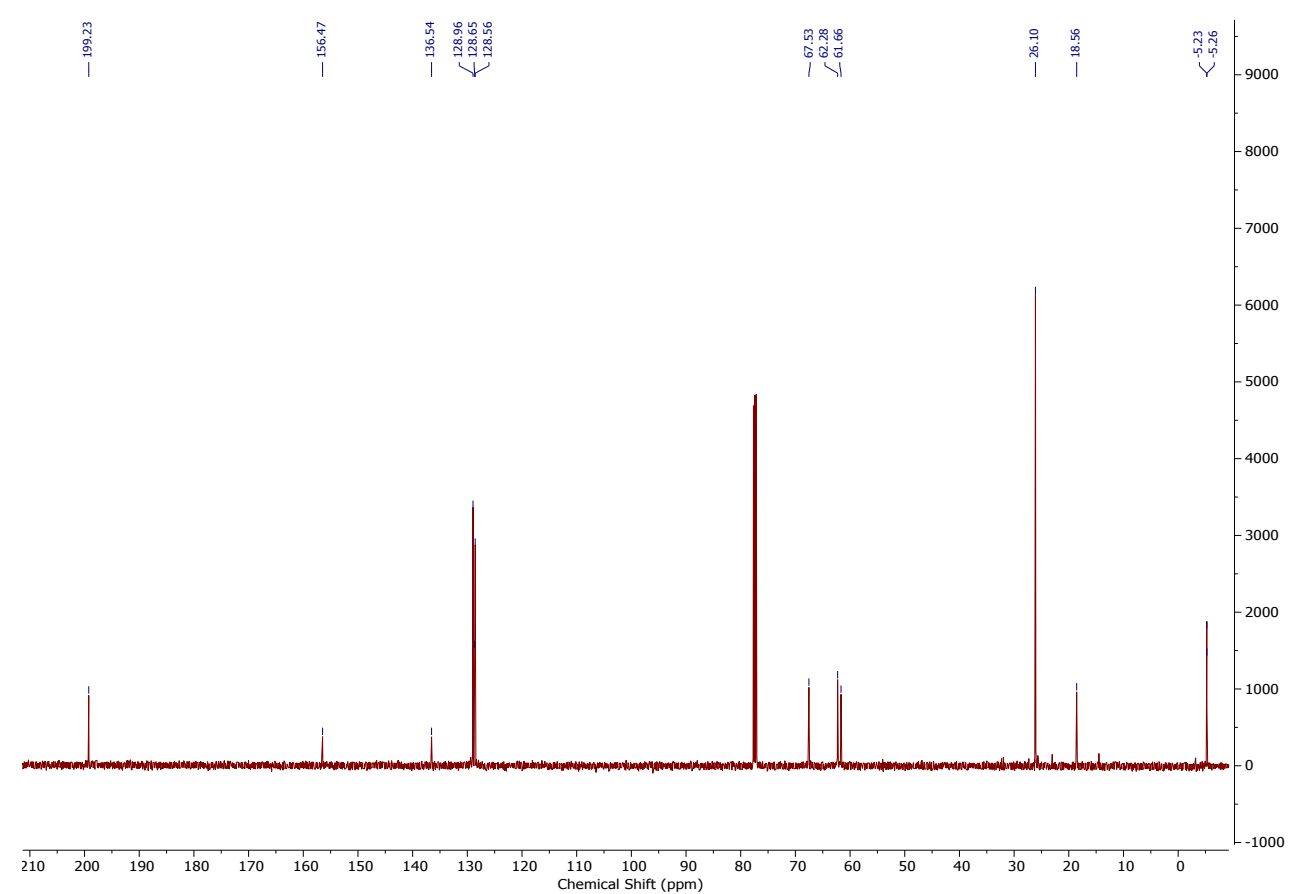


Benzyl ((2S,3S)-1-((tert-butyldimethylsilyl)oxy)-4-(2,2-dimethyl-4-oxo-4H-1,3-dioxin-6yl)-3-hydroxybutan-2-yl)carbamate (37a)<smiles>CC(C)(C)OC[C@H](NC(=O)OCc1ccccc1)[C@@H](O)CC1=CC(=O)OC(C)(C)O1</smiles>

${ }^{1} \mathrm{H}-\mathrm{NMR}\left(500 \mathrm{MHz}, \mathrm{CDCl}_{3}\right)$

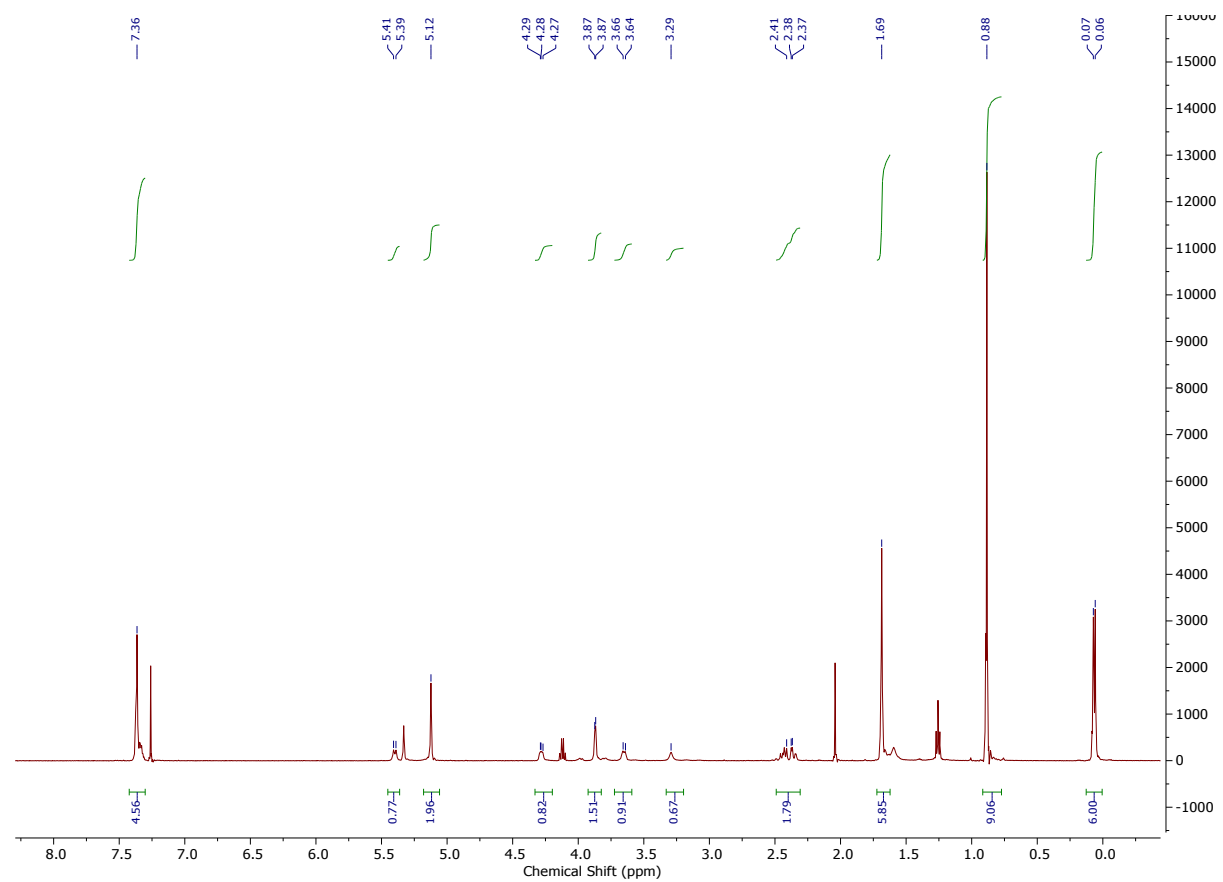

${ }^{13} \mathrm{C}-\mathrm{NMR}\left(126 \mathrm{MHz}, \mathrm{CDCl}_{3}\right)$

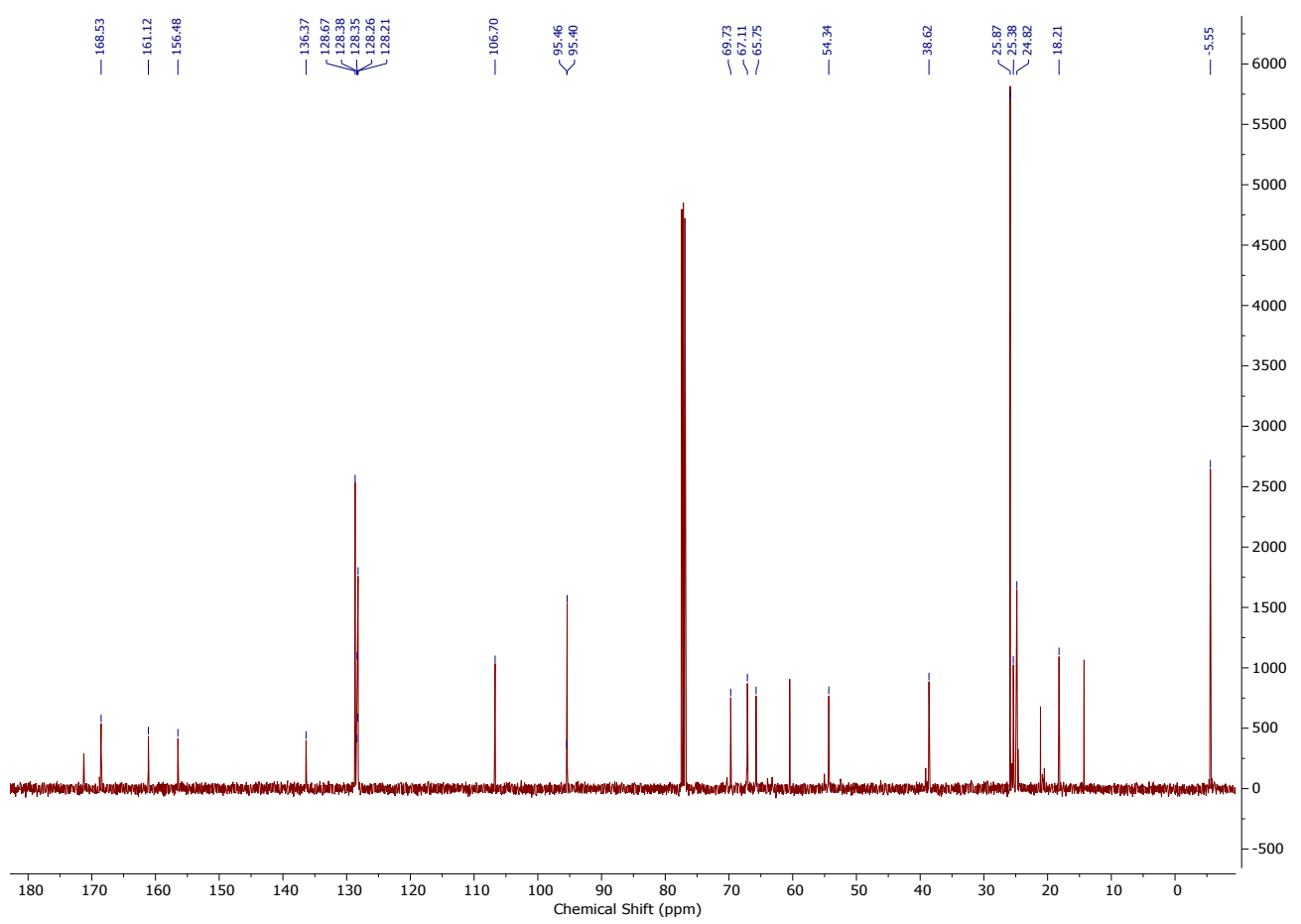


Methyl (5S,6S)-6-(((benzyloxy)carbonyl)amino)-7-((tert-butyldimethylsilyl)oxy)-5-hydroxy3-oxoheptanoate (37)<smiles>COC(=O)CC(=O)CC(O)C(CO[AsH3])NC(=O)OCc1ccccc1</smiles>

${ }^{1} \mathrm{H}-\mathrm{NMR}\left(500 \mathrm{MHz}, \mathrm{CDCl}_{3}\right)$

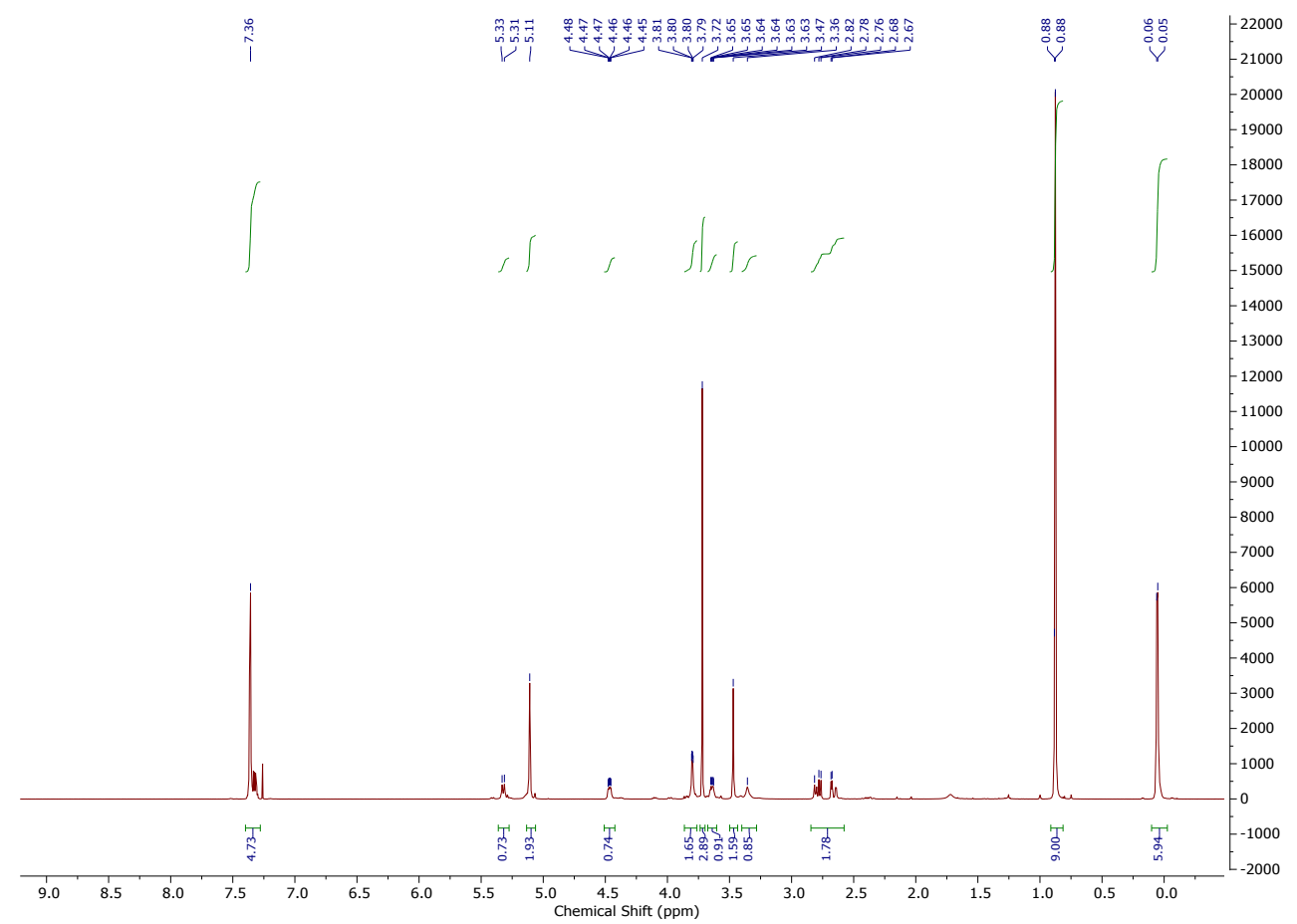

${ }^{13} \mathrm{C}-\mathrm{NMR}\left(126 \mathrm{MHz}, \mathrm{CDCl}_{3}\right)$

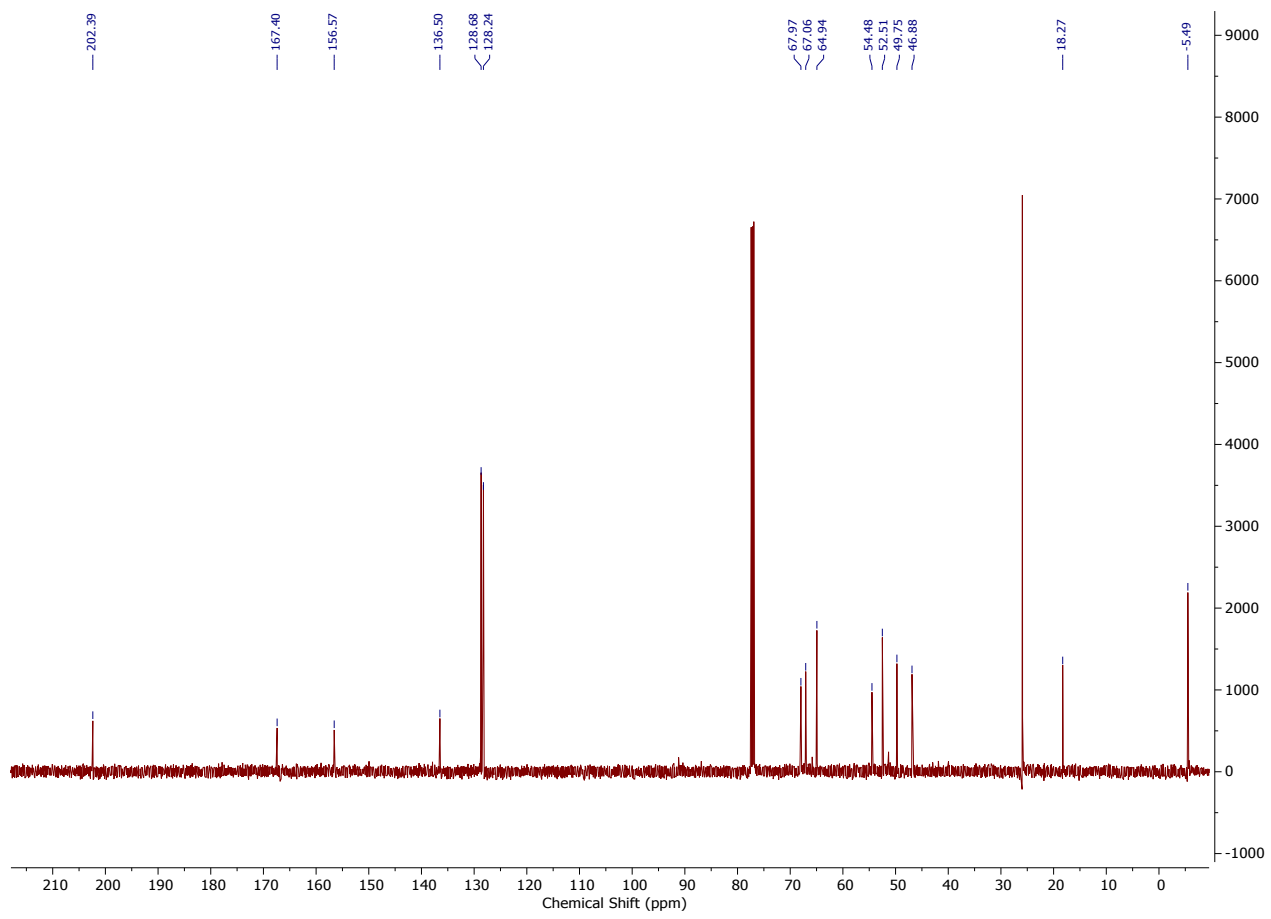


Methyl (3S,5S,6S)-6-(((benzyloxy)carbonyl)amino)-7-((tert-butyldimethylsilyl)oxy)-3,5dihydroxyheptanoate (38)<smiles>COC(=O)CC(O)CC(O)C(CO[As])NC(=O)OCc1ccccc1</smiles>

${ }^{1} \mathrm{H}-\mathrm{NMR}\left(400 \mathrm{MHz}, \mathrm{CDCl}_{3}\right)$

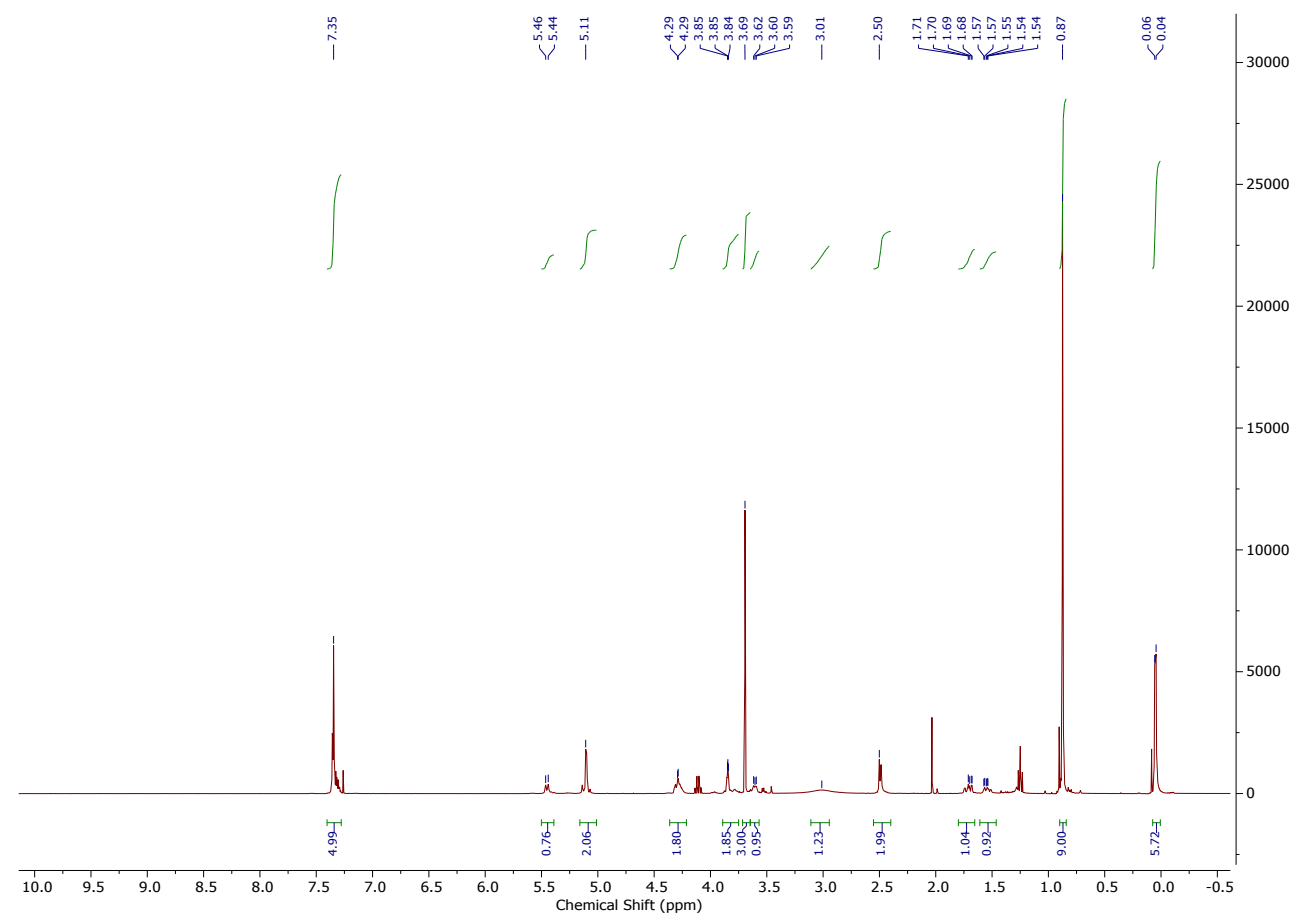

${ }^{13} \mathrm{C}-\mathrm{NMR}\left(100 \mathrm{MHz}, \mathrm{CDCl}_{3}\right)$

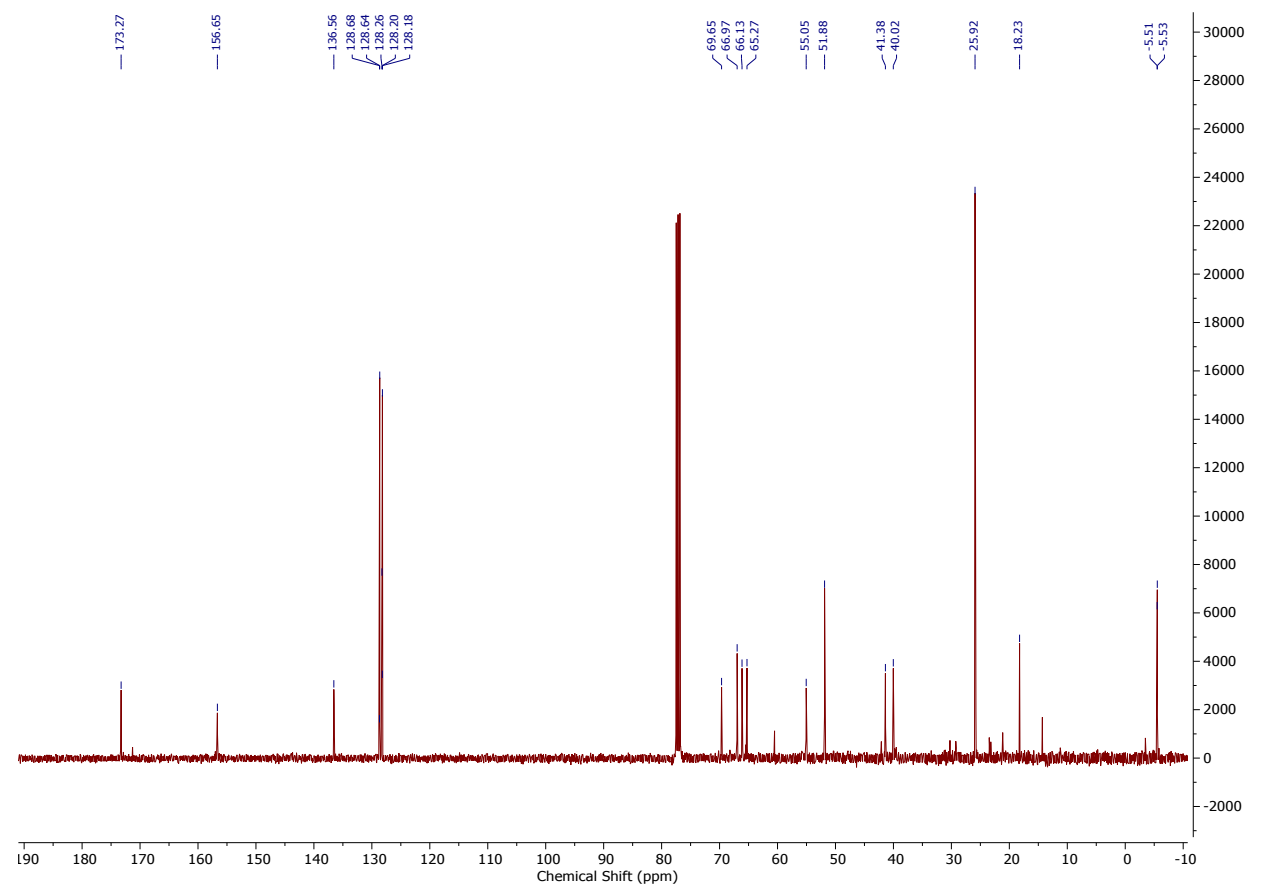


Methyl (3S,5S,6S)-6-(((benzyloxy)carbonyl)amino)-3,5,7-tris((tertbutyldimethylsilyl)oxy)heptanoate (39)

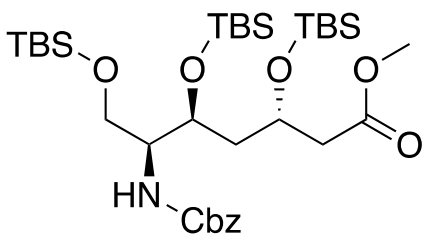

${ }^{1} \mathrm{H}-\mathrm{NMR}\left(500 \mathrm{MHz}, \mathrm{CDCl}_{3}\right)$

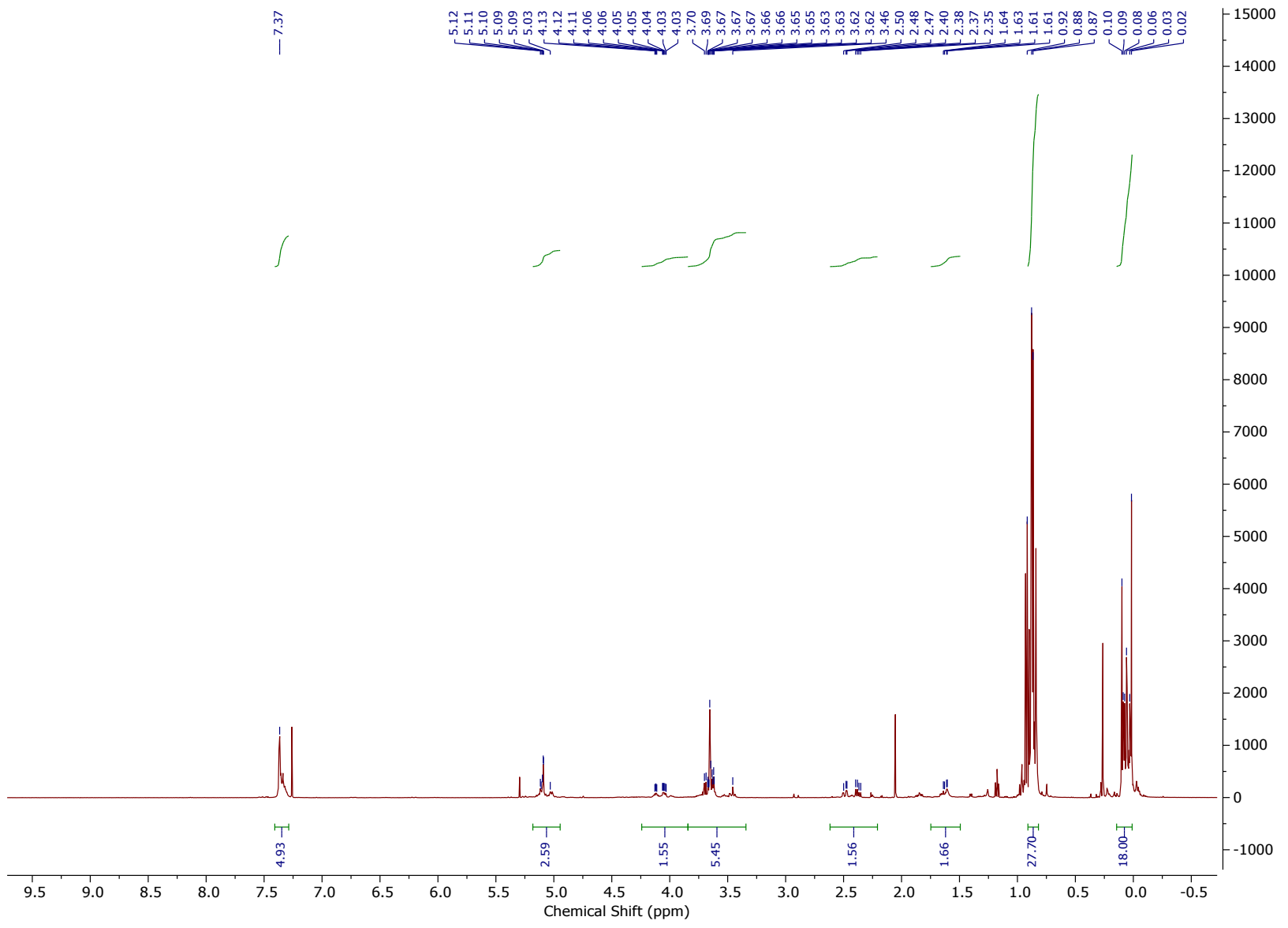


(3S,5S,6S)-6-(((benzyloxy)carbonyl)amino)-3,5,7-tris((tertbutyldimethylsilyl)oxy)heptanoic acid (40)

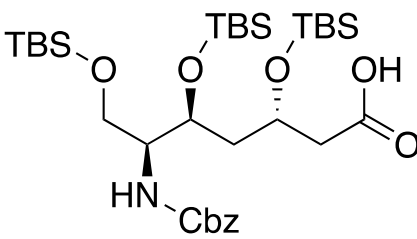

${ }^{1} \mathrm{H}-\mathrm{NMR}\left(500 \mathrm{MHz}, \mathrm{CDCl}_{3}\right)$

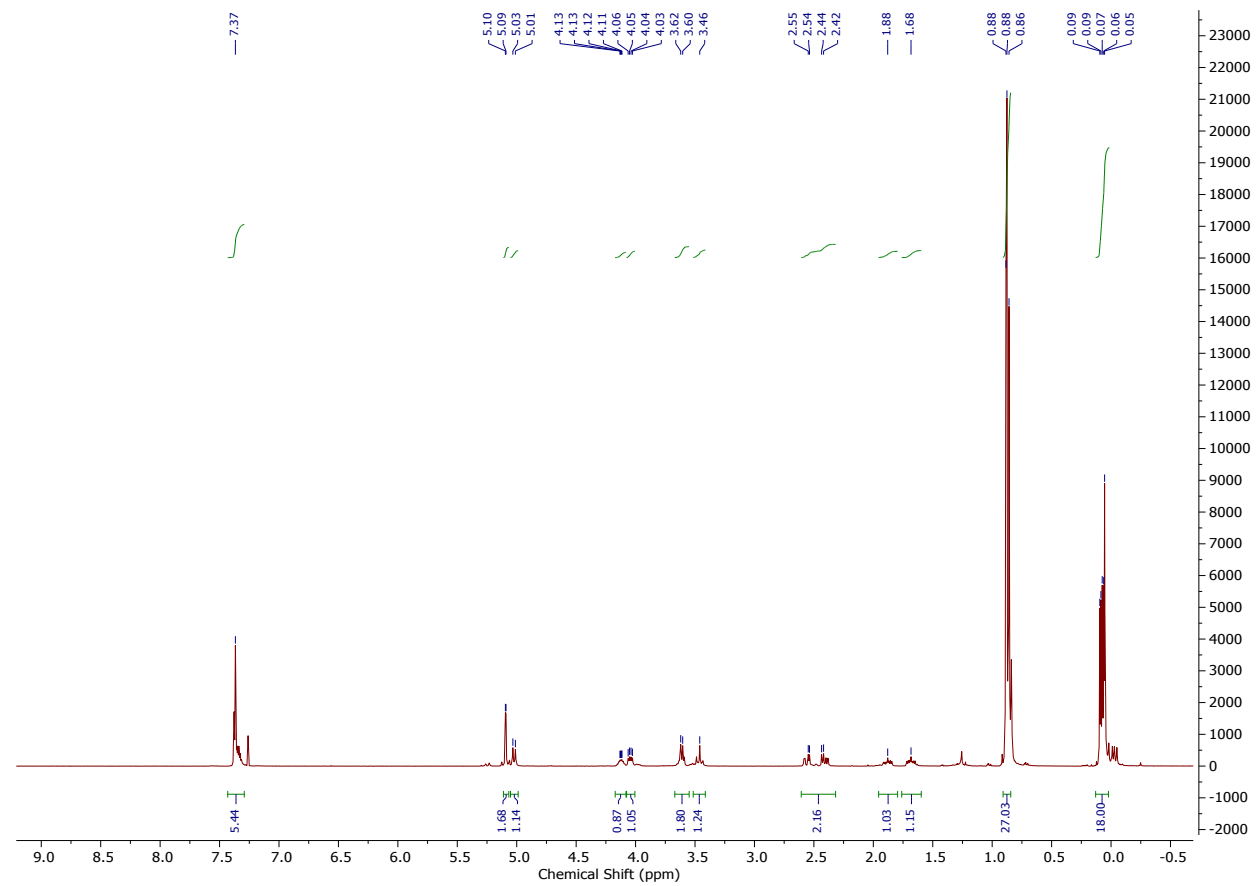

${ }^{13} \mathrm{C}-\mathrm{NMR}\left(126 \mathrm{MHz}, \mathrm{CDCl}_{3}\right)$

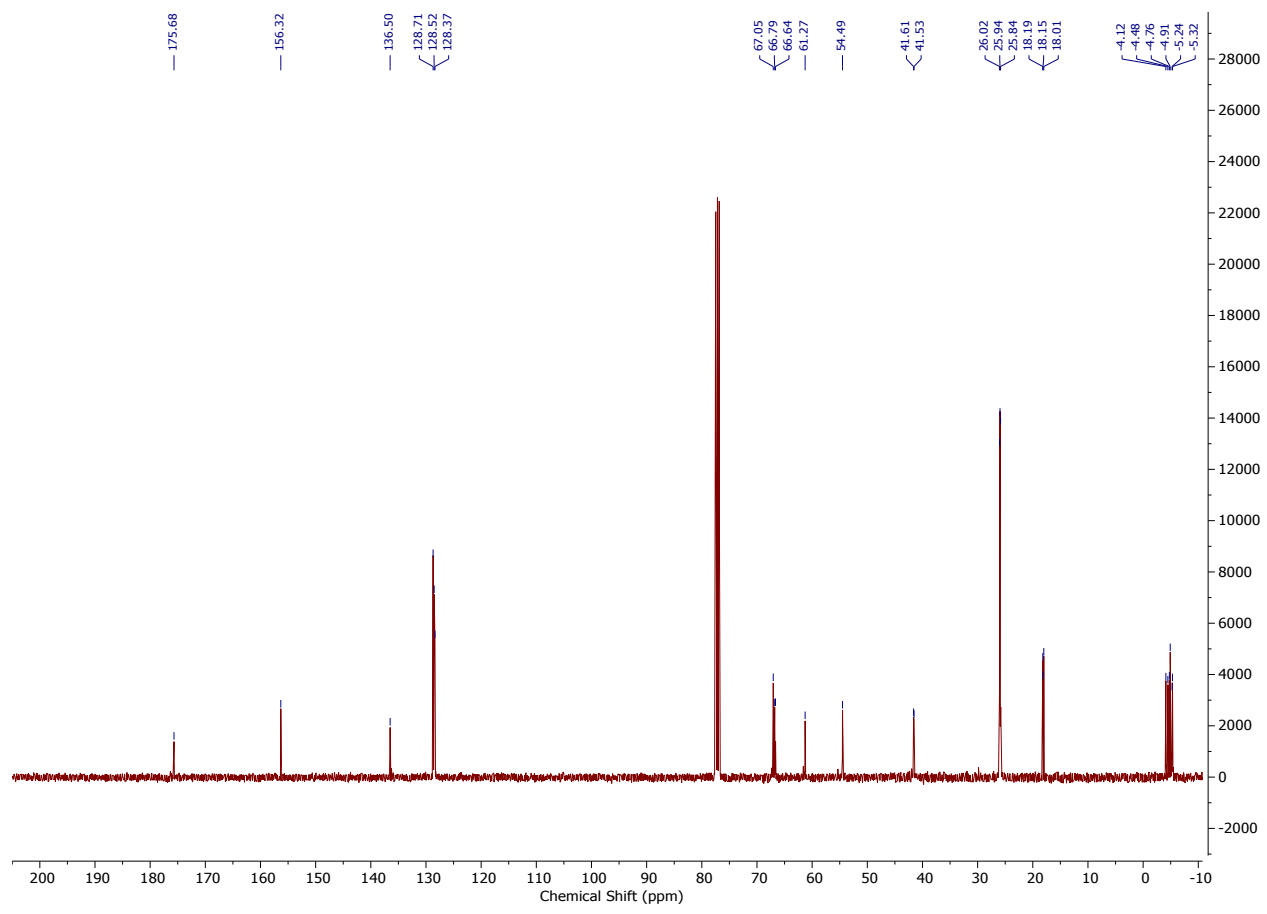


Benzyloxycarbonyl-4-orthonitrobenzesulfonyl-diaminobutane (2)<smiles>CC(C)(C)OC(=O)NCCCCCNS(C)(=O)=O</smiles>

${ }^{1} \mathrm{H}-\mathrm{NMR}\left(400 \mathrm{MHz}, \mathrm{CDCl}_{3}\right)$

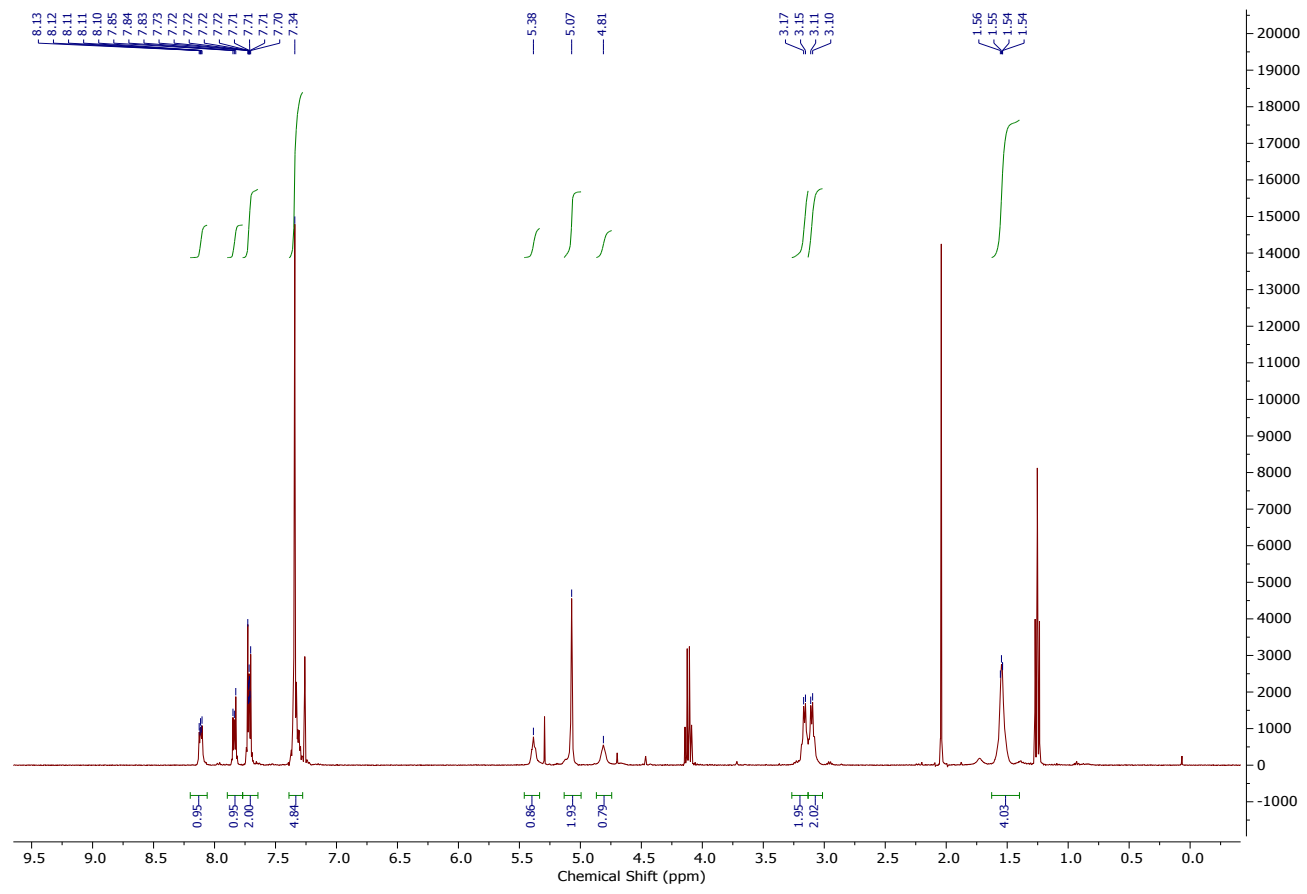

${ }^{13} \mathrm{C}-N M R\left(100 \mathrm{MHz}, \mathrm{CDCl}_{3}\right)$

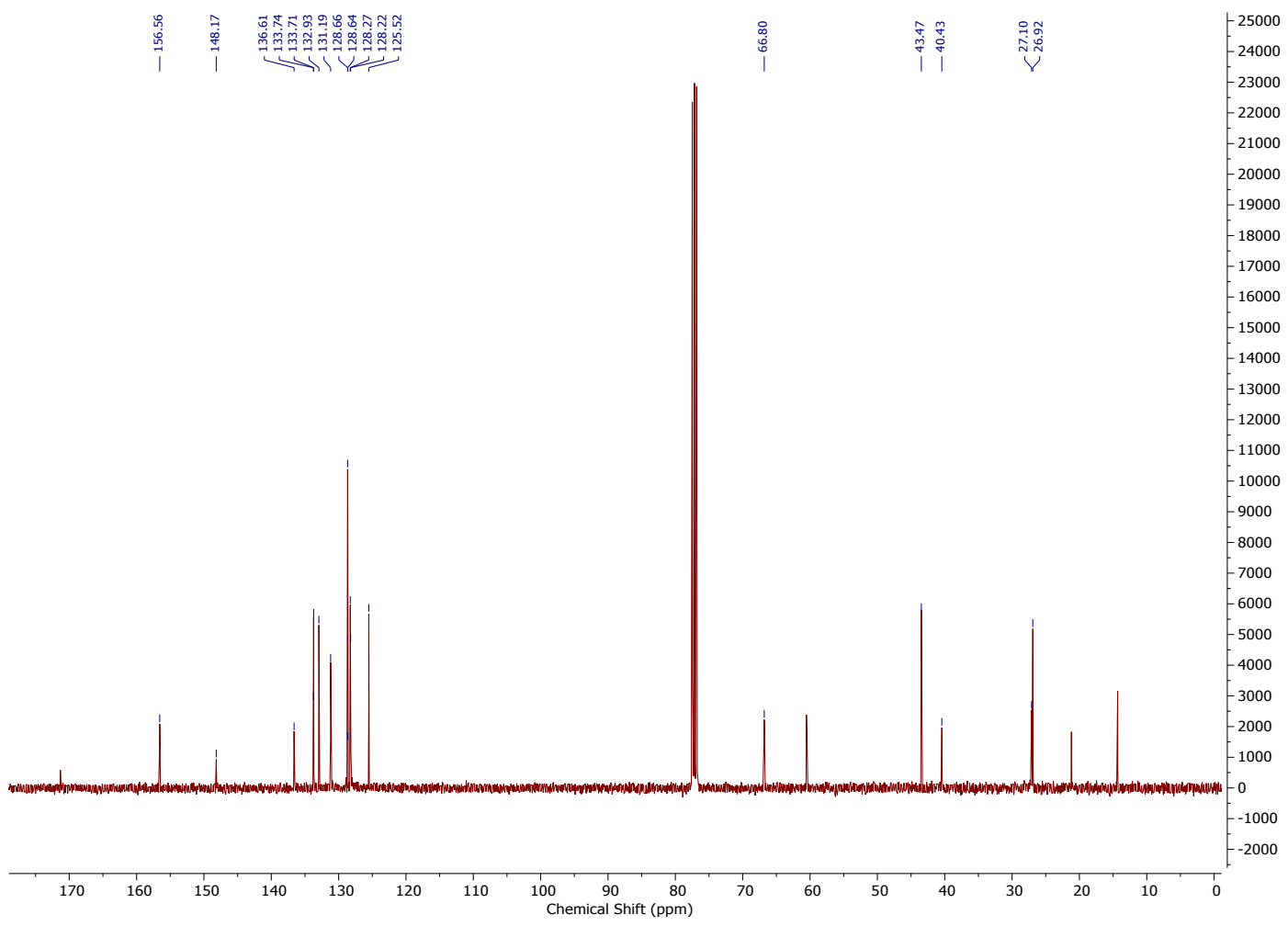




\section{$N^{1}$-Cbz- $N^{4}$-oNs- $N^{8}$-Boc-spermidine (3)}<smiles>CC(C)(C)OC(=O)NCCCN(CCCCNC(=O)OCc1ccccc1)NS(C)(=O)=O</smiles>

${ }^{1} \mathrm{H}-\mathrm{NMR}\left(400 \mathrm{MHz}, \mathrm{CDCl}_{3}\right)$

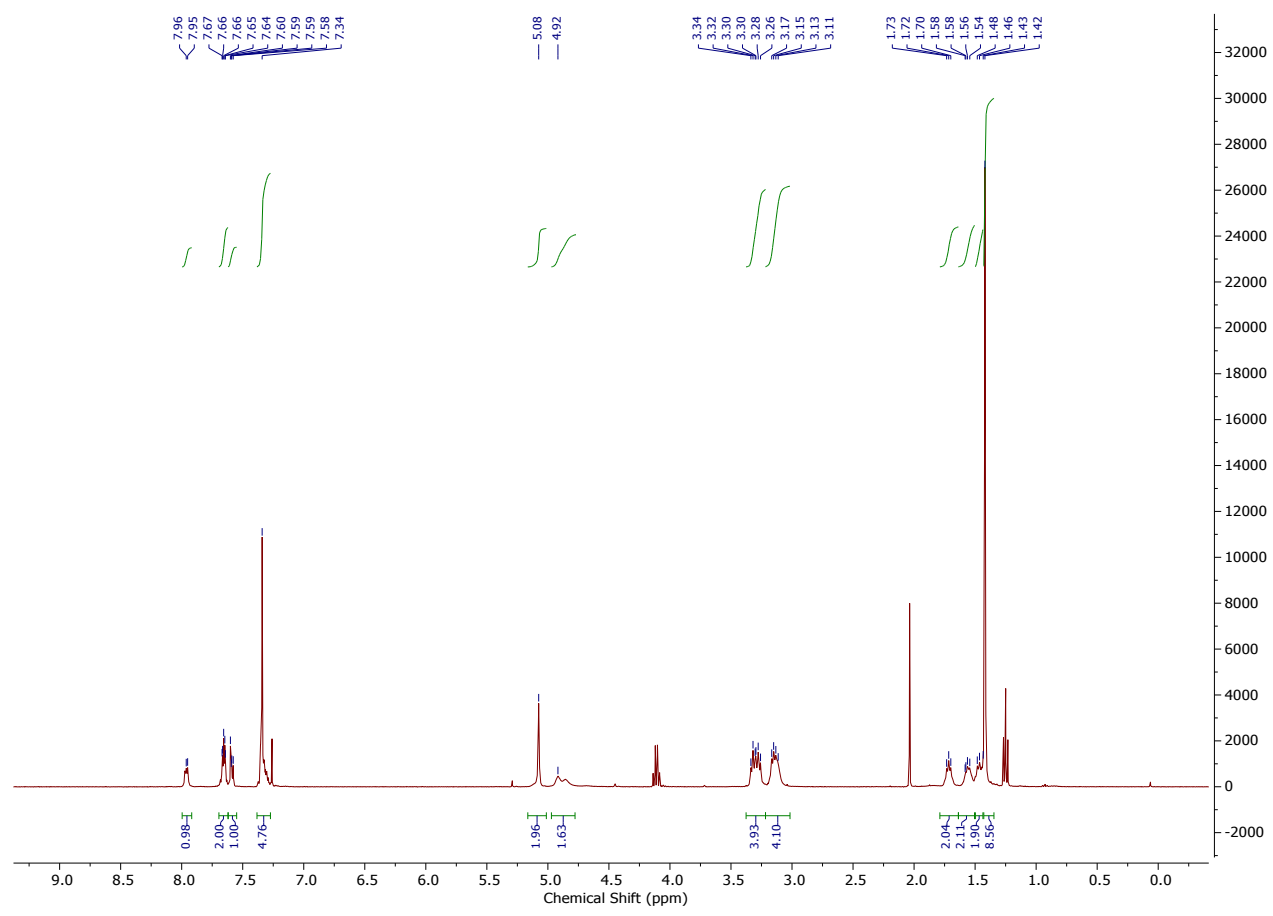

${ }^{13} \mathrm{C}-\mathrm{NMR}\left(100 \mathrm{MHz}, \mathrm{CDCl}_{3}\right)$

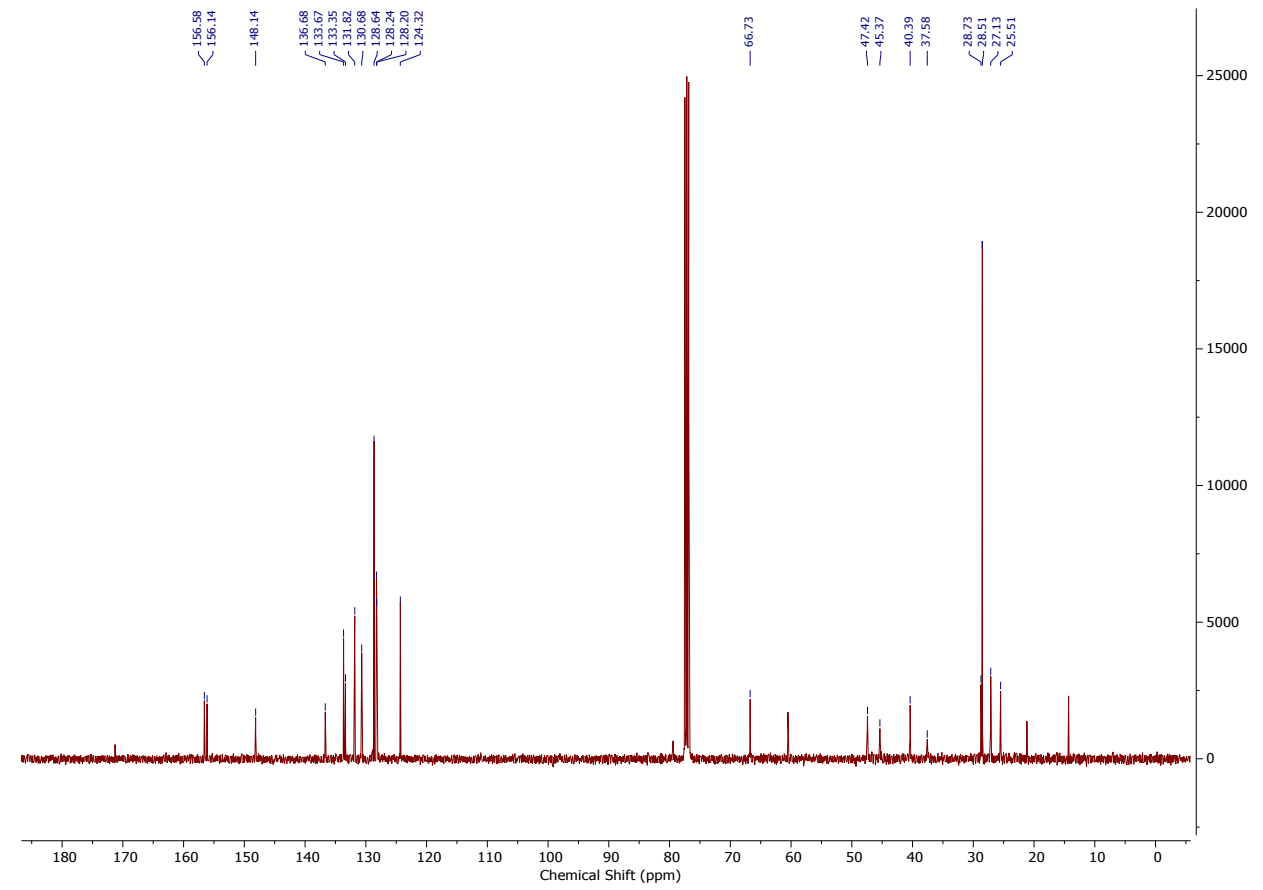




\section{$N^{1}$-Cbz- $N^{4}, N^{8}$-bis-Boc-spermidine (3a)}<smiles>CC(C)(C)OC(=O)NCCCN(CCCCNC(=O)OCc1ccccc1)C(=O)OC(C)(C)C</smiles>

${ }^{1} \mathrm{H}-\mathrm{NMR}\left(400 \mathrm{MHz}, \mathrm{CDCl}_{3}\right)$

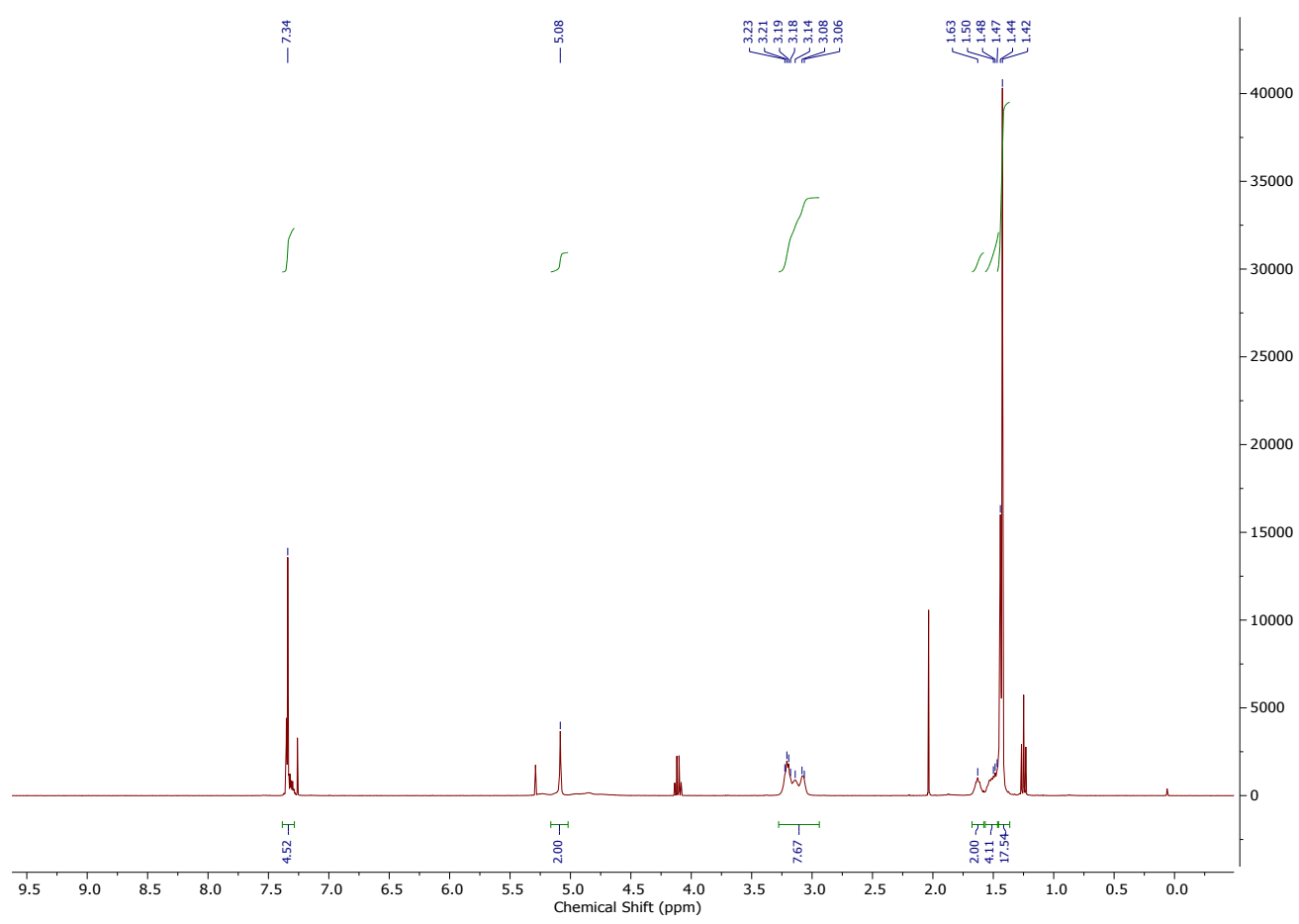

${ }^{13} \mathrm{C}-\mathrm{NMR}\left(100 \mathrm{MHz}, \mathrm{CDCl}_{3}\right)$

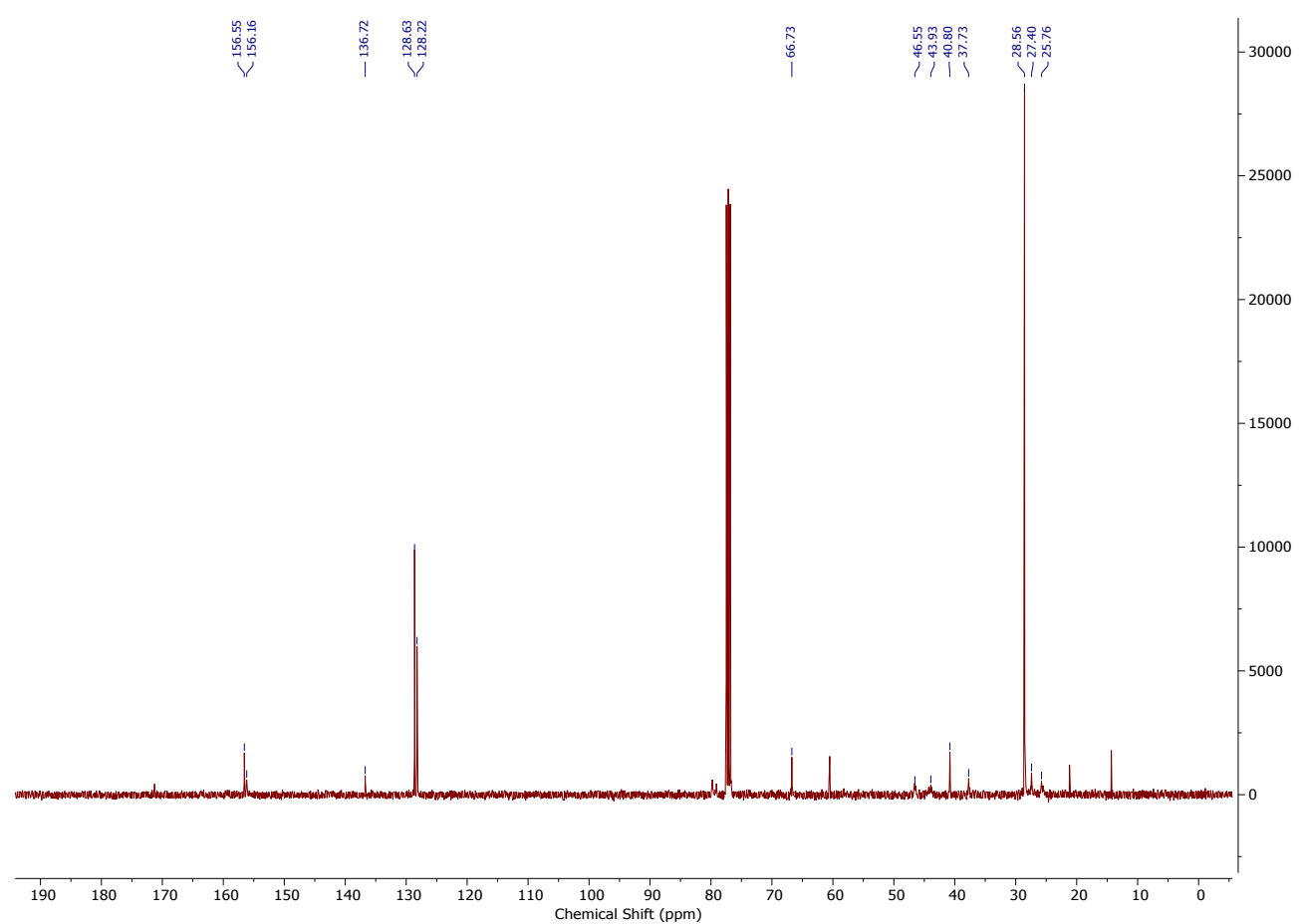


$N^{4}, N^{8}$-bis-Boc-spermidine (4)<smiles>CC(C)(C)OC(=O)NCCCN(CCCCN)C(=O)OC(C)(C)C</smiles>

${ }^{1} \mathrm{H}-\mathrm{NMR}(400 \mathrm{MHz}$, DMSO-d 6 )

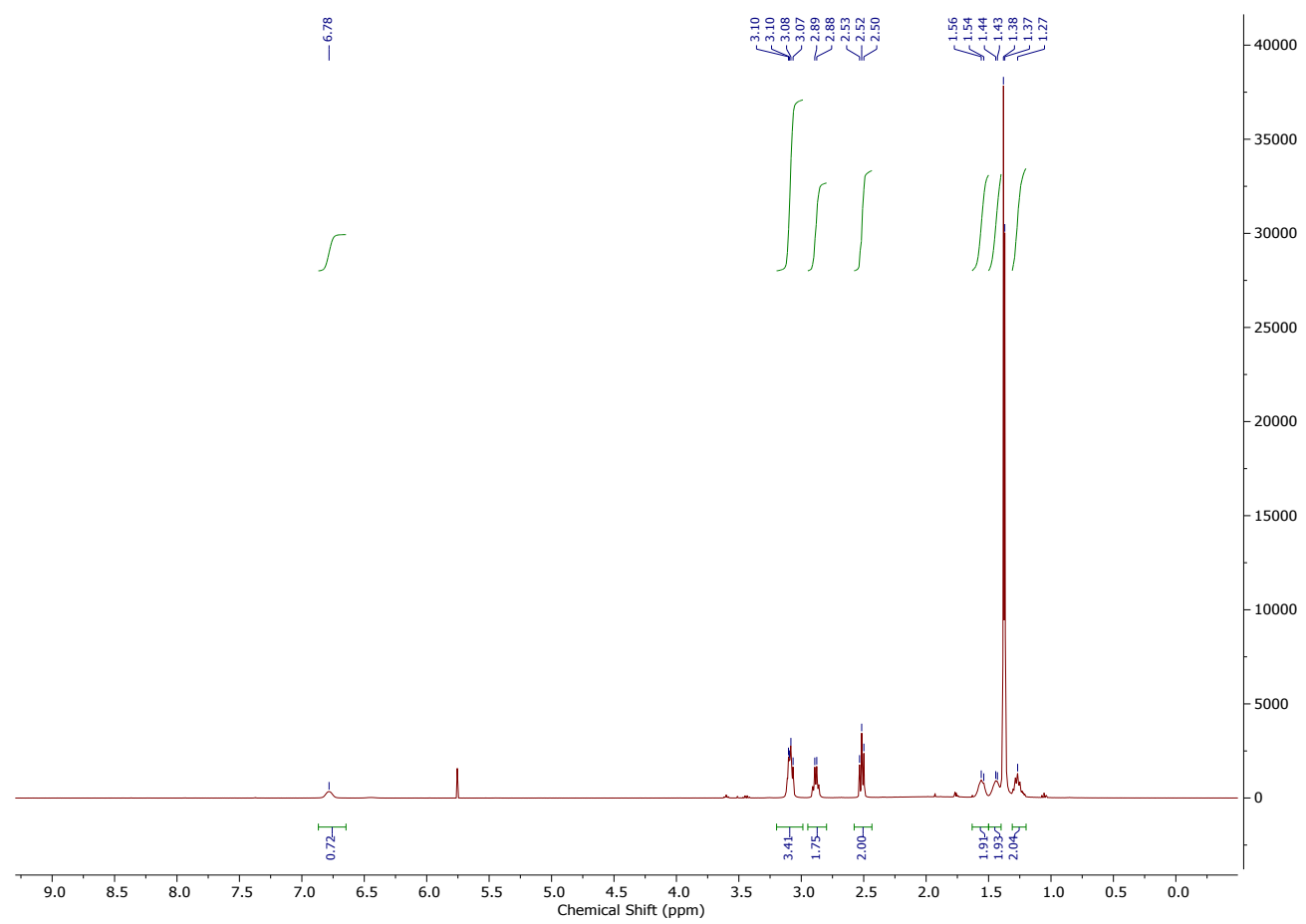

${ }^{13} \mathrm{C}-\mathrm{NMR}(100 \mathrm{MHz}$, DMSO-d 6 )

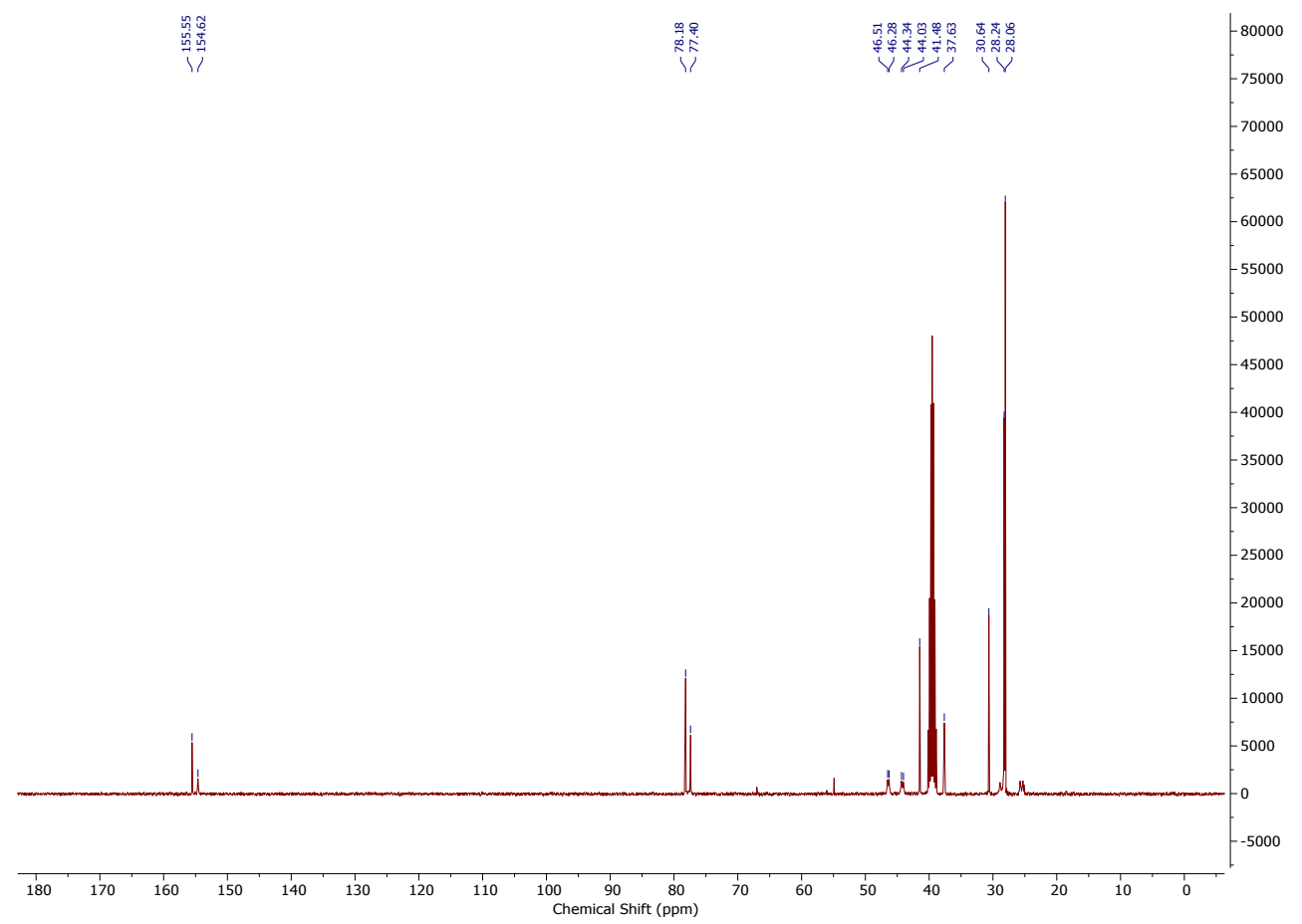


Cbz-L-DAP-OH (6)<smiles>NC[C@H](NC(=O)OCc1ccccc1)C(=O)O</smiles>

${ }^{1} \mathrm{H}-\mathrm{NMR}\left(500 \mathrm{MHz}, \mathrm{D}_{2} \mathrm{O} / \mathrm{DMSO}-\mathrm{d}_{6}+1 \% \mathrm{TFA}\right)$

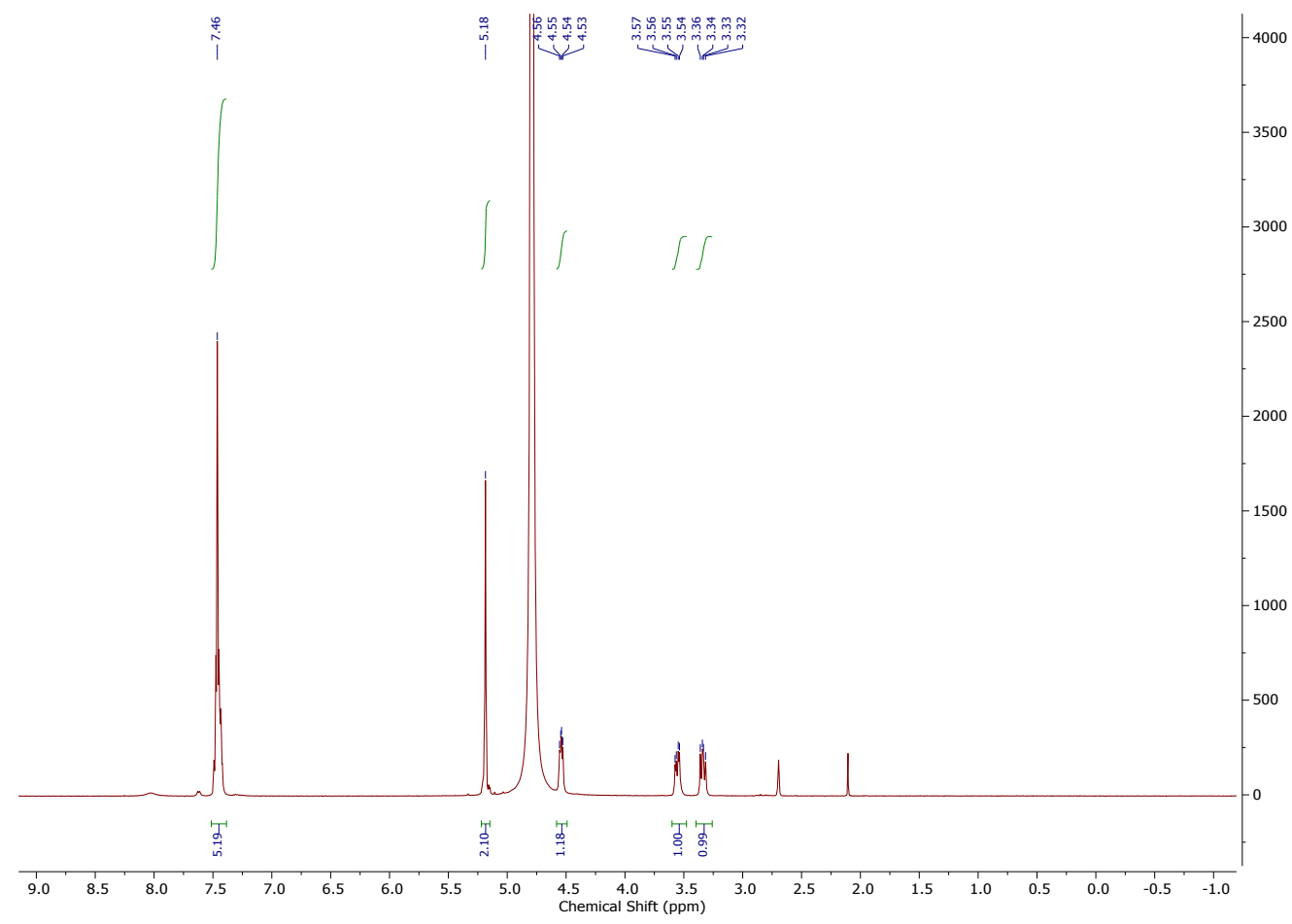

${ }^{13} \mathrm{C}-N M R\left(126 \mathrm{MHz}, \mathrm{D}_{2} \mathrm{O} / \mathrm{DMSO}-\mathrm{d}_{6}+1 \%\right.$ TFA)

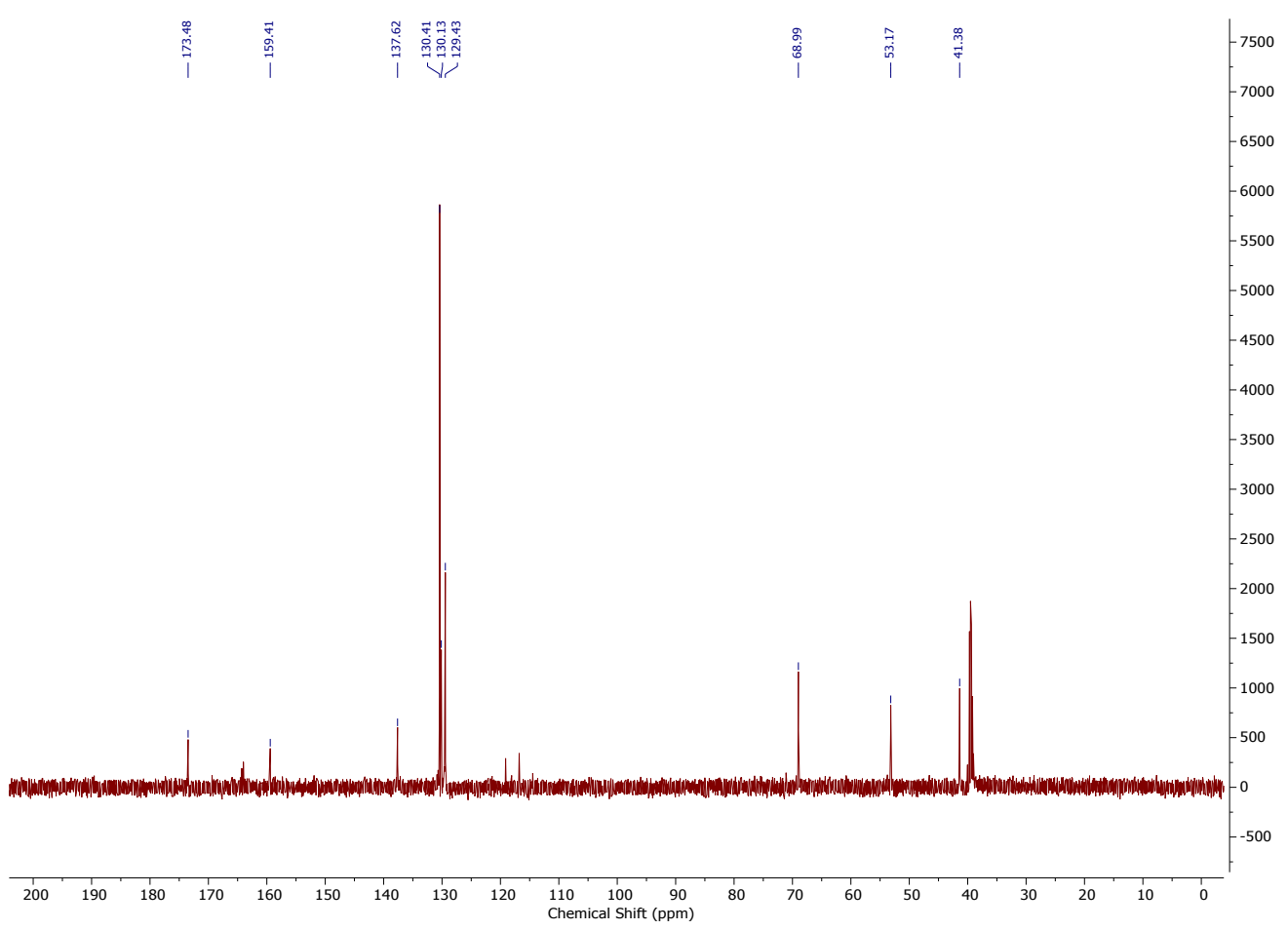


Cbz-L-mDAP(oNs)-OMe (7)<smiles>COC(=O)C(CN(C)S(=O)(=O)c1ccccc1[N+](=O)[O-])NC(=O)OCc1ccccc1</smiles>

${ }^{1} \mathrm{H}-\mathrm{NMR}\left(500 \mathrm{MHz}, \mathrm{CDCl}_{3}\right)$

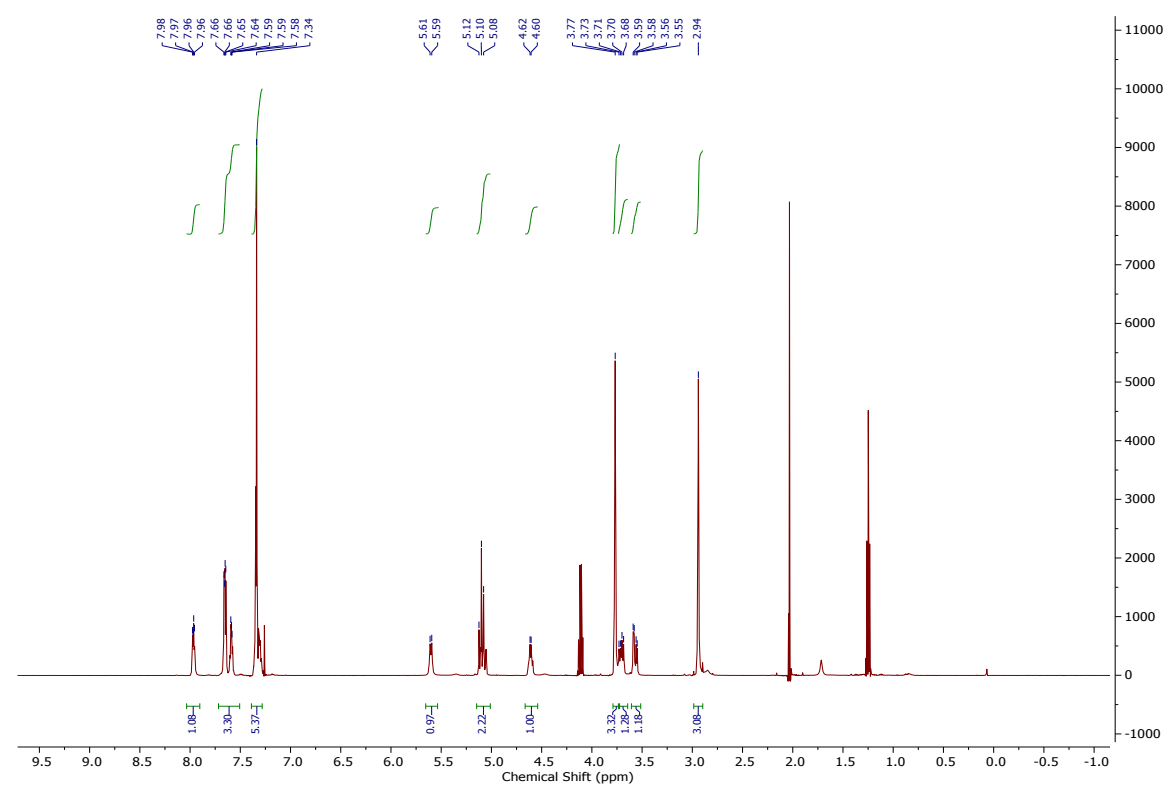

${ }^{13} \mathrm{C}-\mathrm{NMR}\left(500 \mathrm{MHz}, \mathrm{CDCl}_{3}\right)$

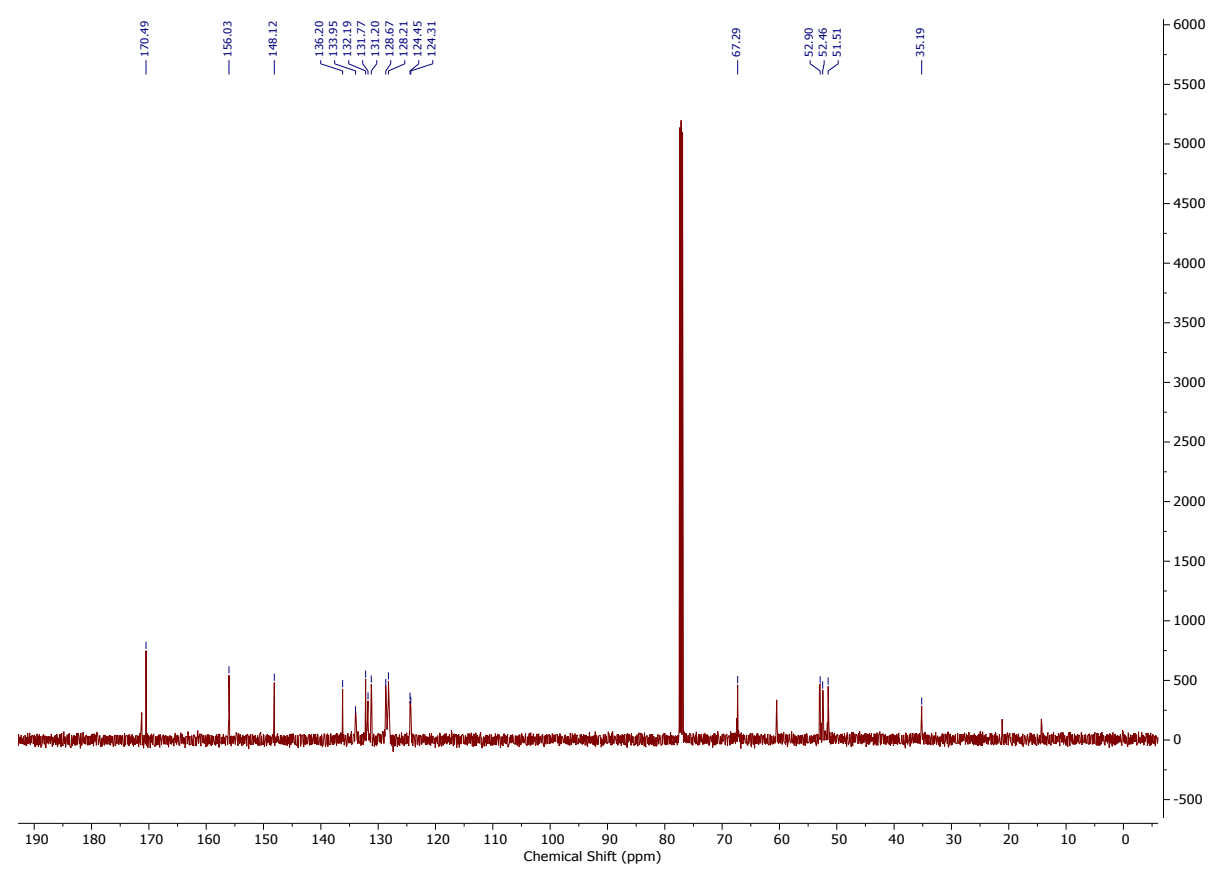


Cbz-L-mDAP(Boc)-OH (8)<smiles>CN(C[C@H](NC(=O)OCc1ccccc1)C(=O)O)C(=O)O</smiles>

${ }^{1} \mathrm{H}-\mathrm{NMR}(500 \mathrm{MHz}$, DMSO-d 6 )

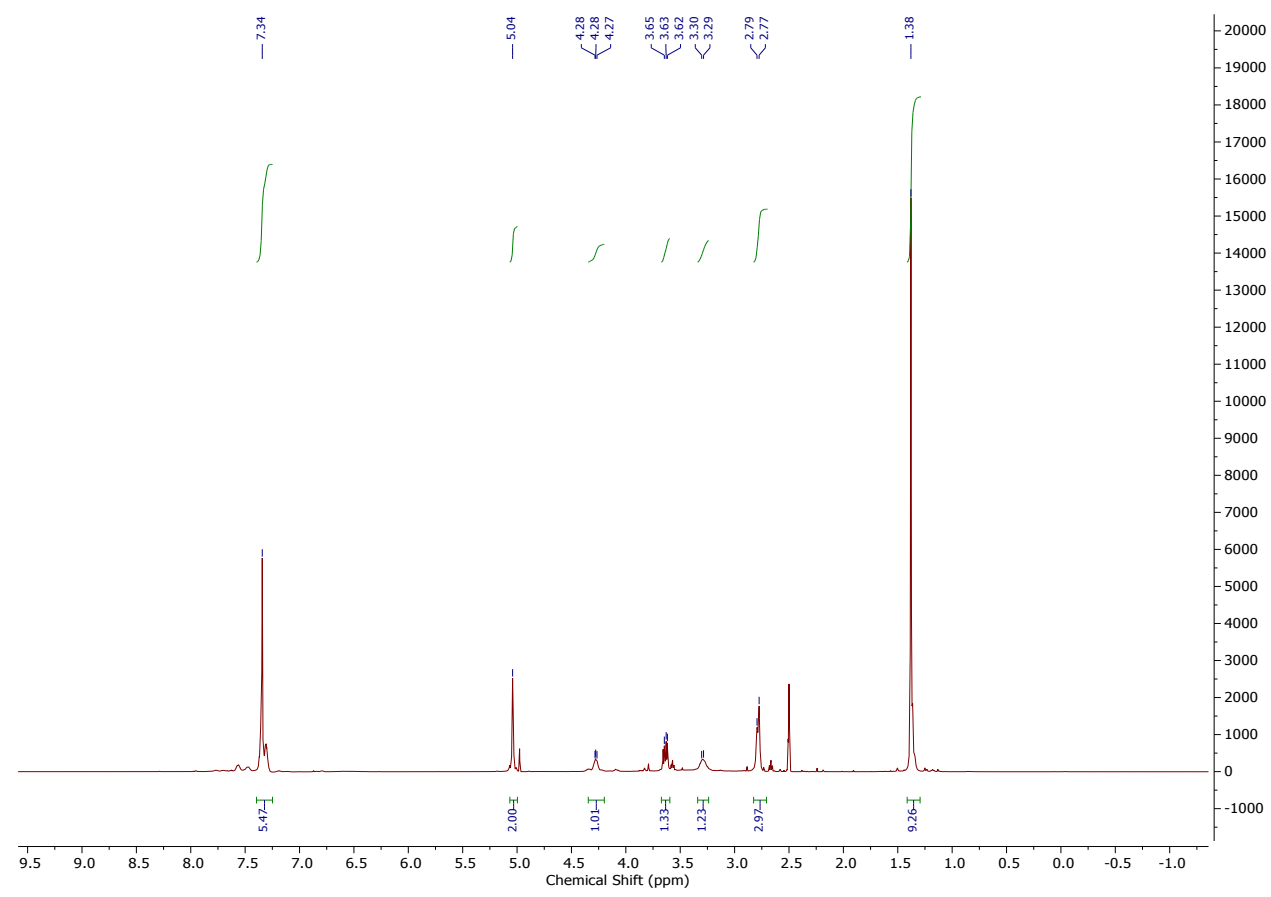

${ }^{13} \mathrm{C}-\mathrm{NMR}$ (126 MHz, DMSO-d $\mathrm{d}_{6}$ )

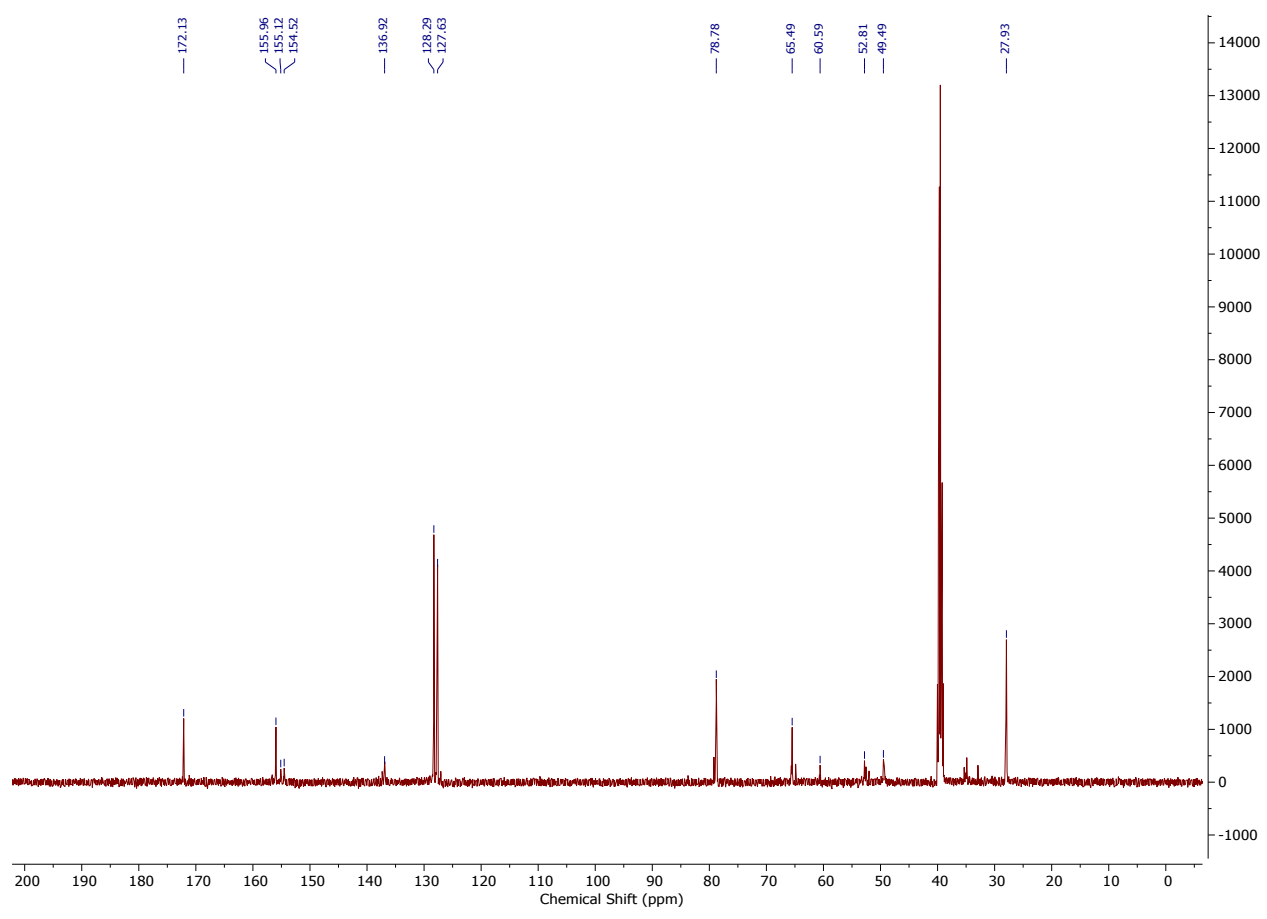


Cbz-D-Ala-L-mDAP(Boc)-D-Orn(Boc)-OMe (28)

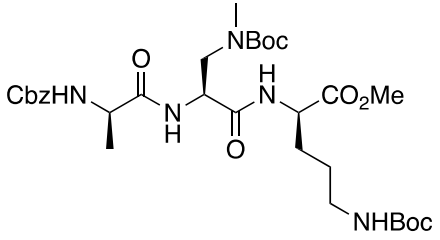

${ }^{1} \mathrm{H}-\mathrm{NMR}\left(500 \mathrm{MHz}, \mathrm{CDCl}_{3}\right)$

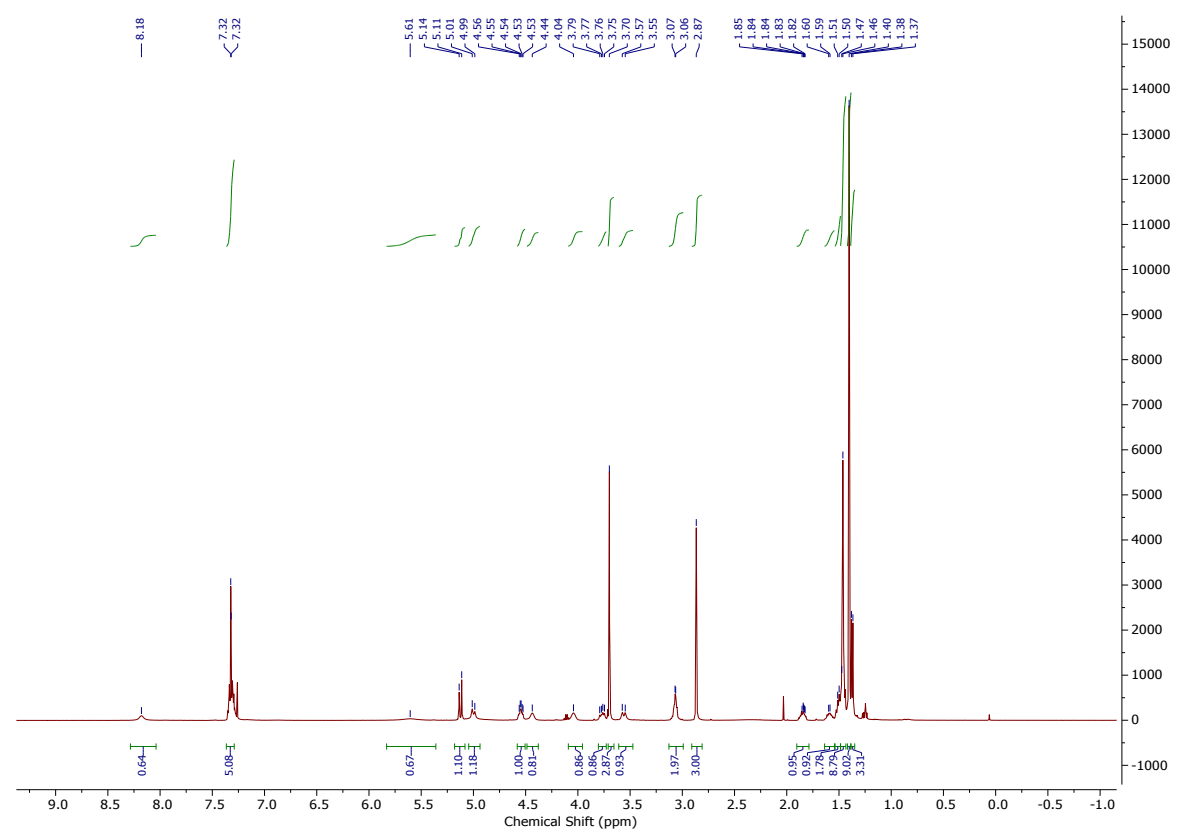

${ }^{13} \mathrm{C}-\mathrm{NMR}\left(126 \mathrm{MHz}, \mathrm{CDCl}_{3}\right)$

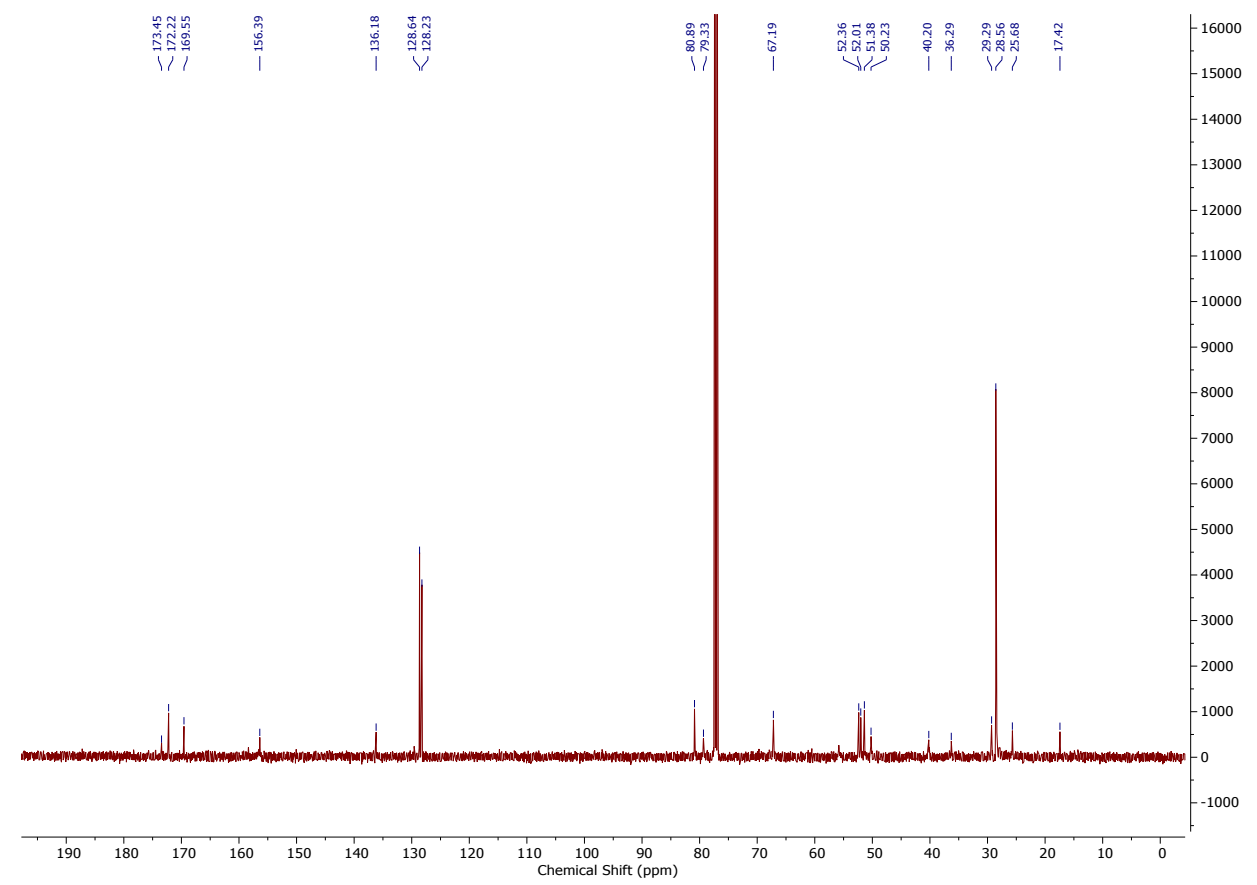


$\mathrm{Boc}_{3}$-Agm-Hpa(diacetonid)-D-Ala-L-mDAP(Boc)-D-Orn(Boc)-OH (LHS)

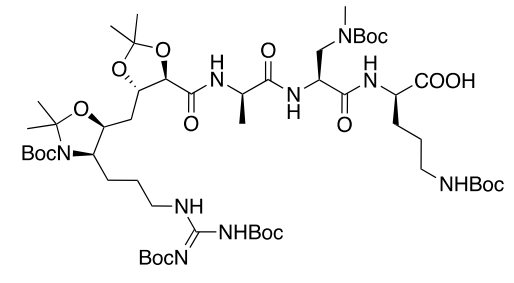

${ }^{1} \mathrm{H}-\mathrm{NMR}\left(400 \mathrm{MHz}, \mathrm{CDCl}_{3}\right)$

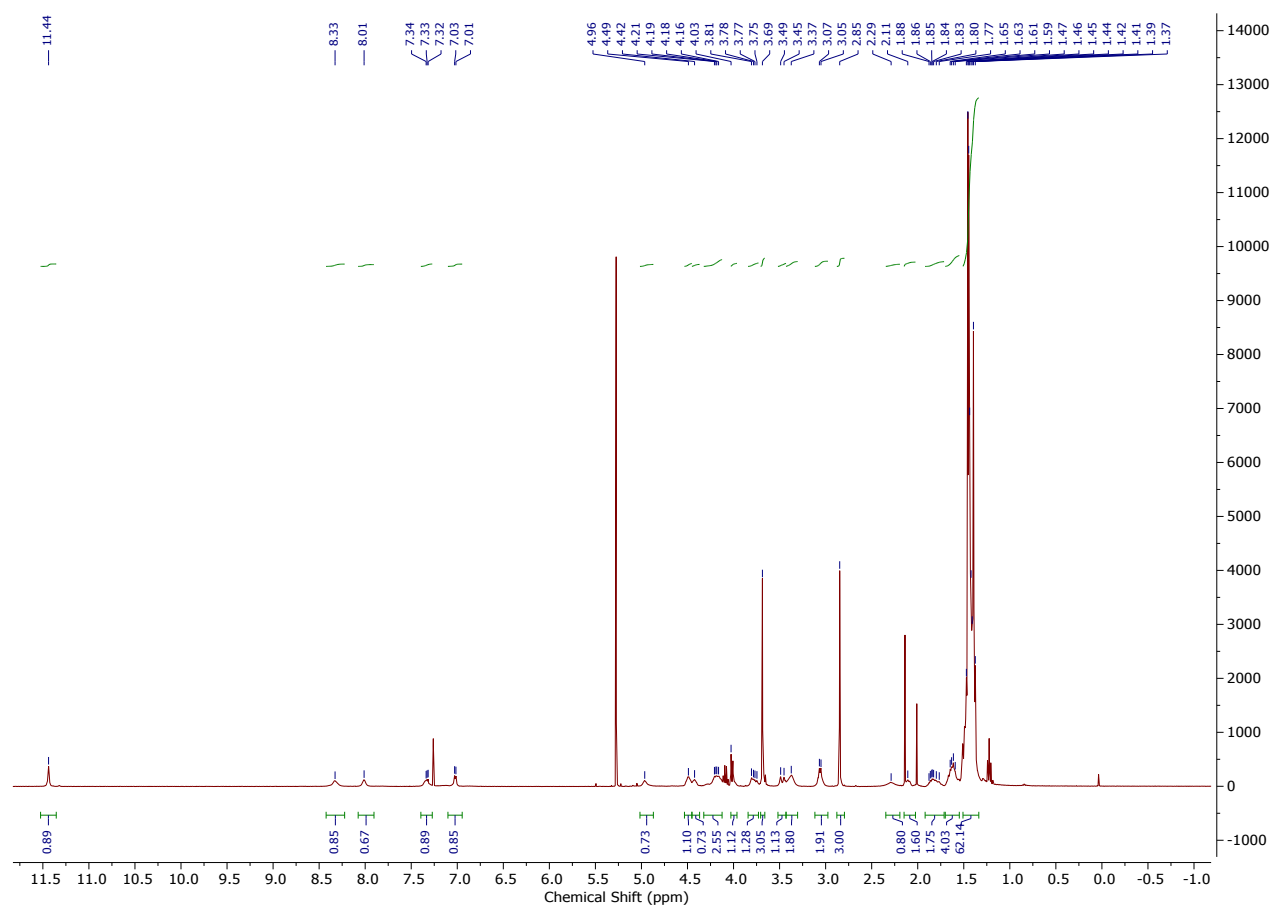

${ }^{13} \mathrm{C}-\mathrm{NMR}\left(100 \mathrm{MHz}, \mathrm{CDCl}_{3}\right)$

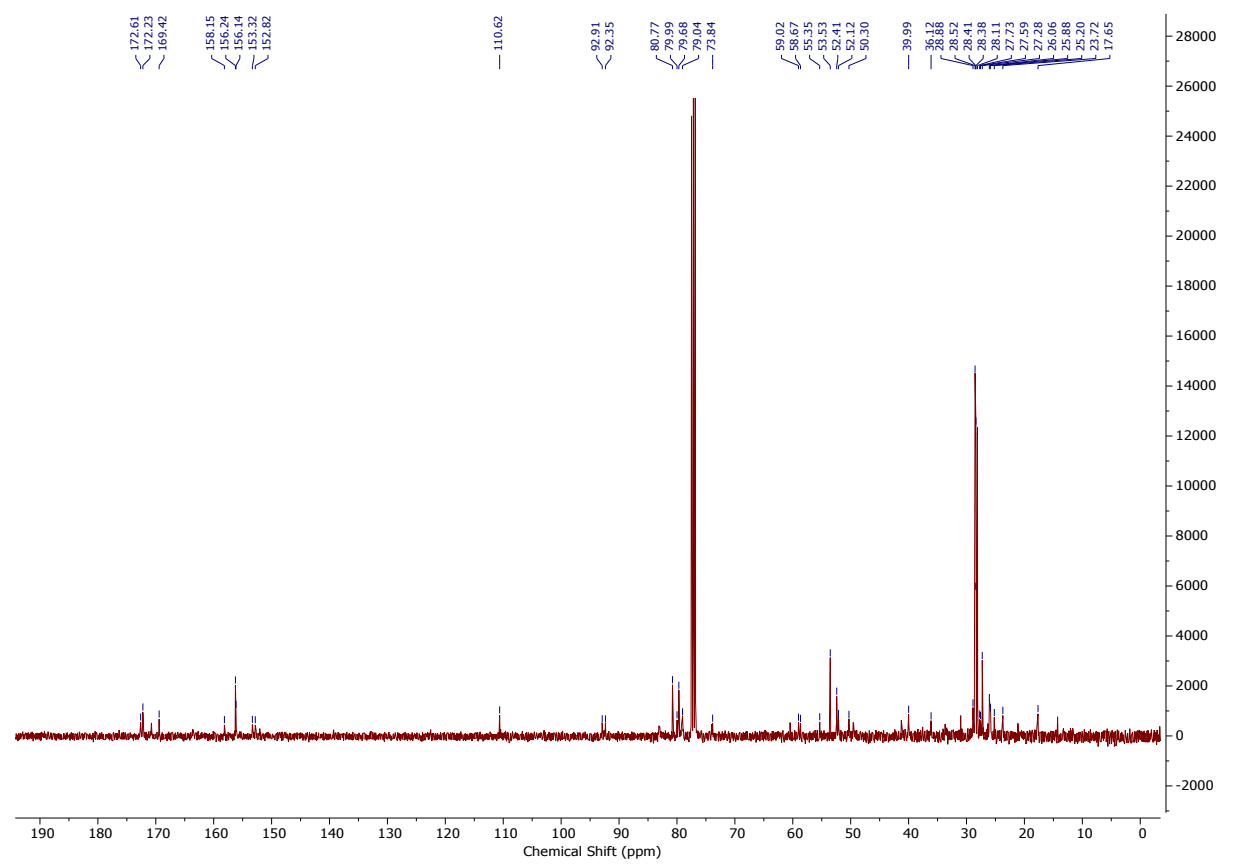




\section{Cbz-L-mDAP(Boc)-Gly-OH}

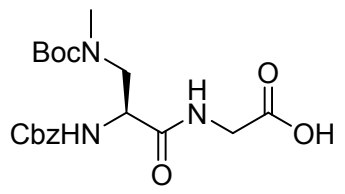

${ }^{1} \mathrm{H}-\mathrm{NMR}\left(500 \mathrm{MHz}, \mathrm{CDCl}_{3}\right)$

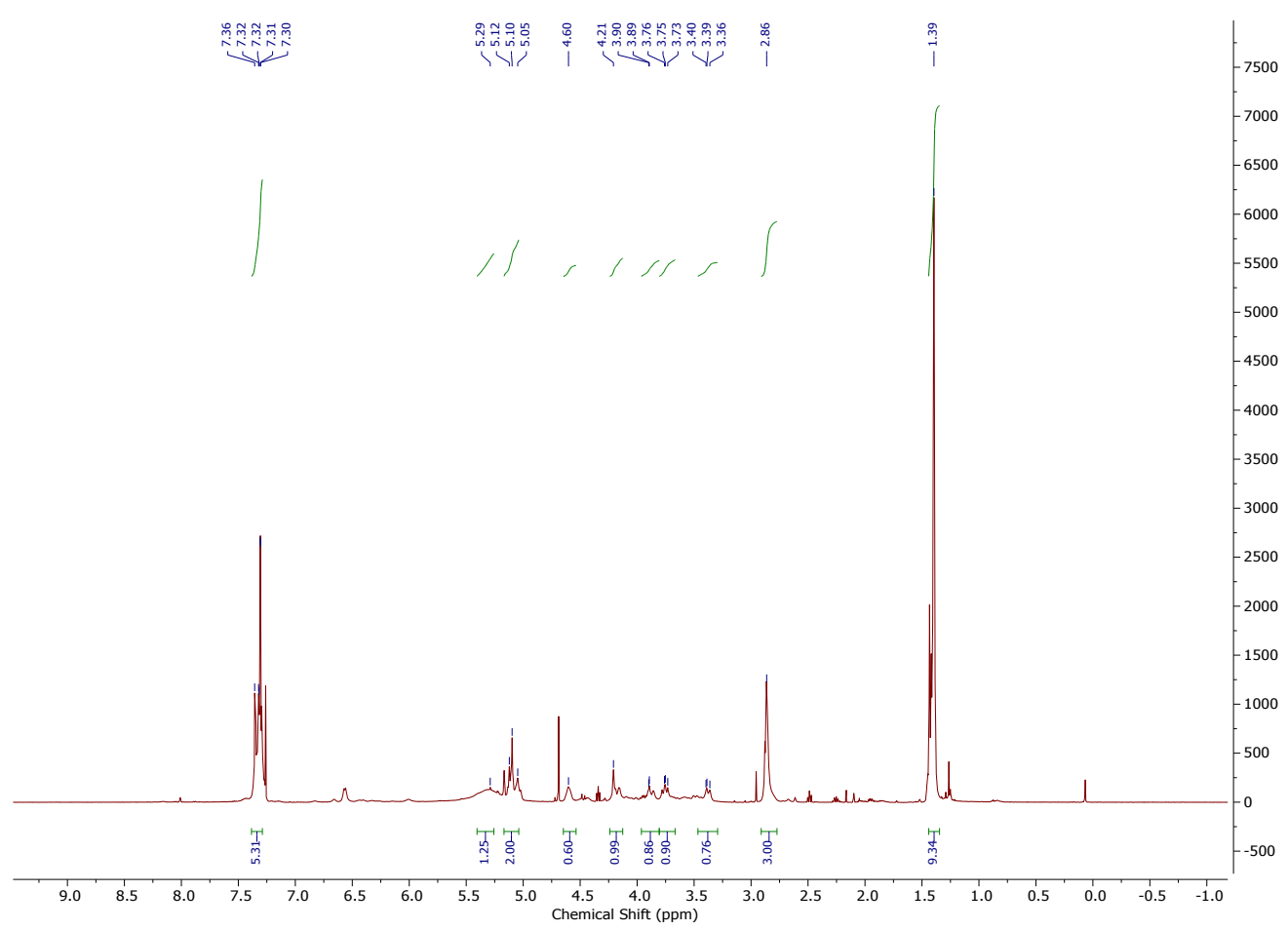

${ }^{13} \mathrm{C}-\mathrm{NMR}\left(126 \mathrm{MHz}, \mathrm{CDCl}_{3}\right)$

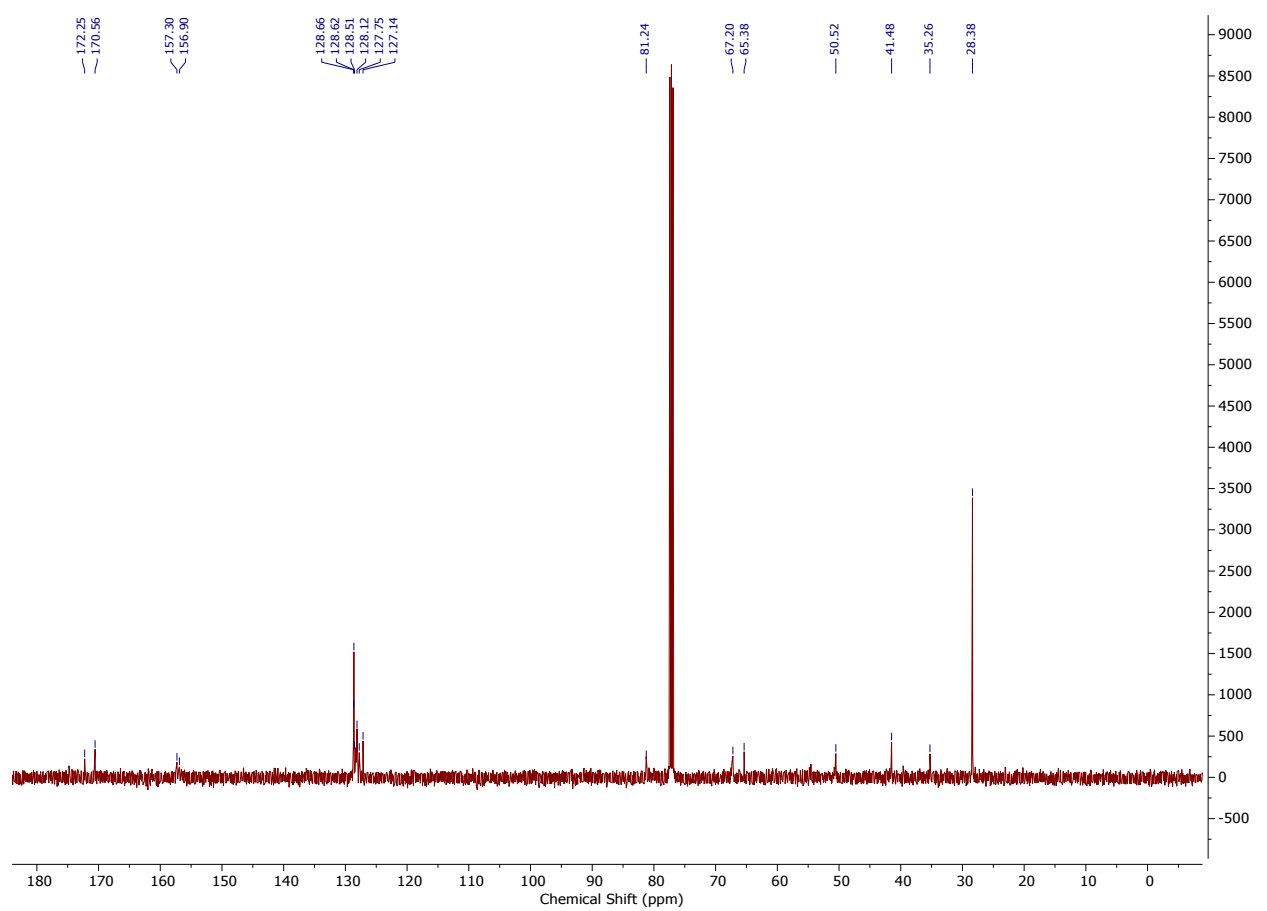


$\mathrm{NH}_{2}$-L-mDAP(Boc)-Gly-4,3-Spd(Boc) ${ }_{2}$<smiles>CN(CC(=O)OC(C)(C)C)C[C@H](N)C(=O)NCC(=O)NCCCCNC(=O)OC(C)(C)C</smiles>

${ }^{1} \mathrm{H}-\mathrm{NMR}\left(500 \mathrm{MHz}, \mathrm{CDCl}_{3}\right)$

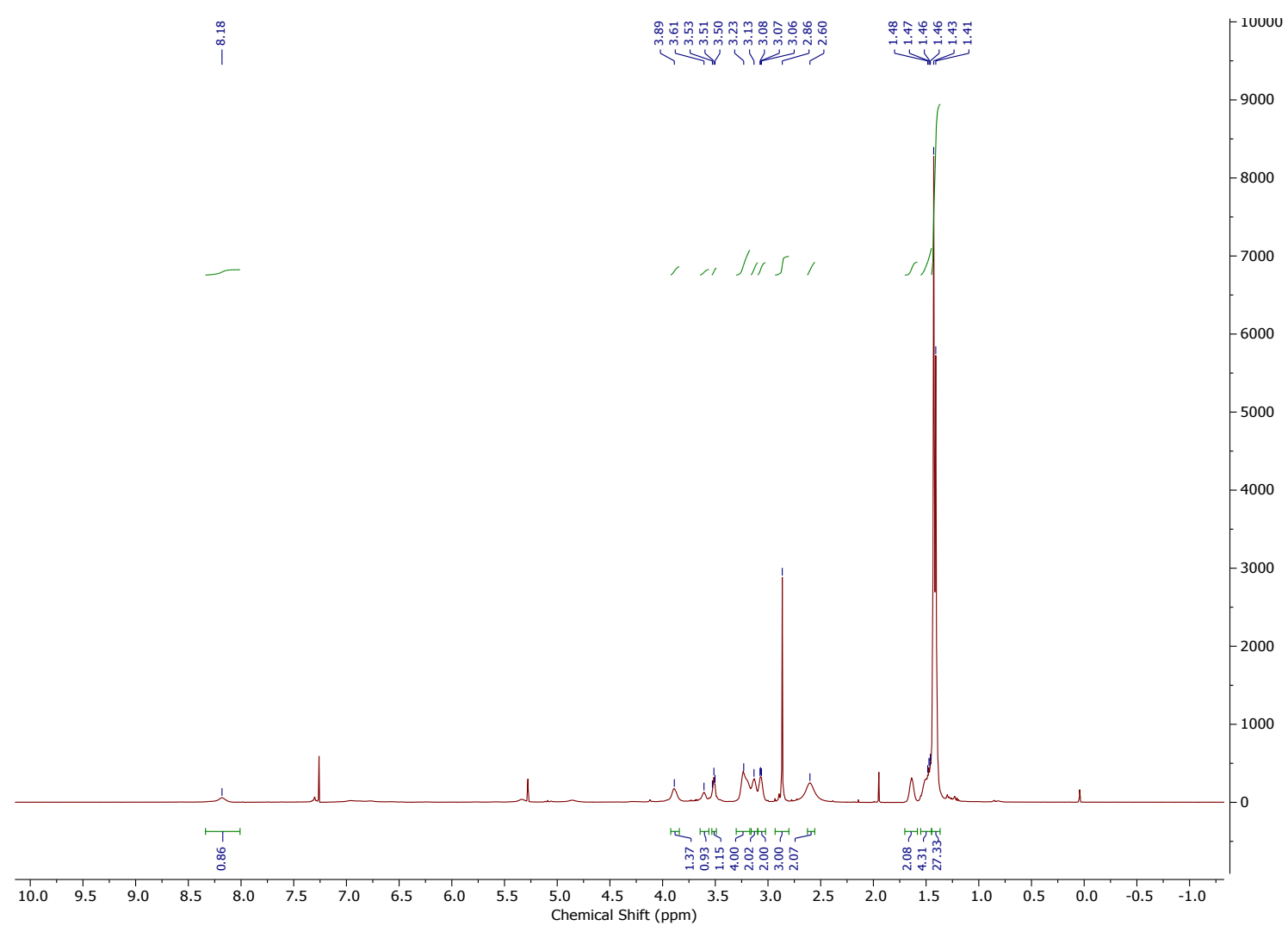


Cbz-(3S,5S,6S)-Gla(7-TBDMS, 3,5-0,0-TBMS)-L-mDAP(Boc)-Gly-4,3-Spd(Boc) ${ }_{2}$ (RHS)

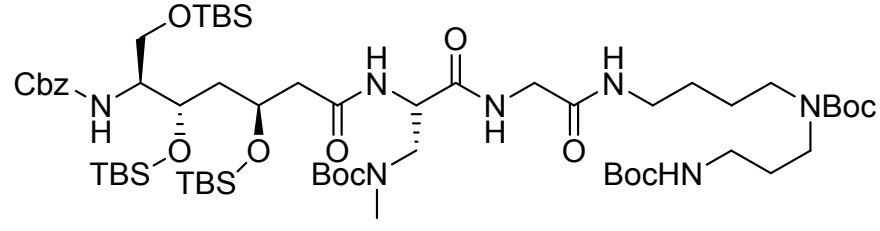

${ }^{1} \mathrm{H}-\mathrm{NMR}\left(400 \mathrm{MHz}, \mathrm{CDCl}_{3}\right)$

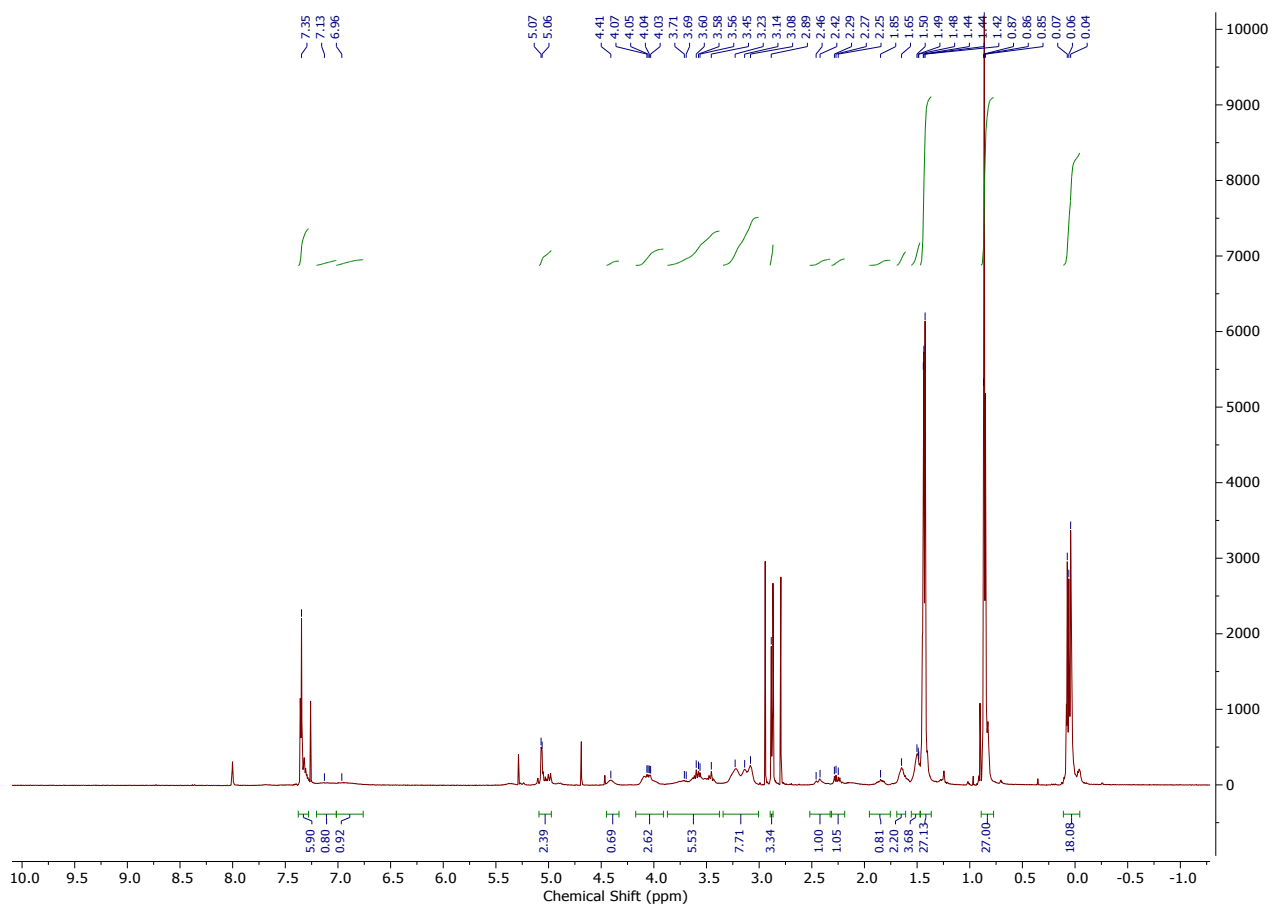

${ }^{13} \mathrm{C}-\mathrm{NMR}\left(100 \mathrm{MHz}, \mathrm{CDCl}_{3}\right)$

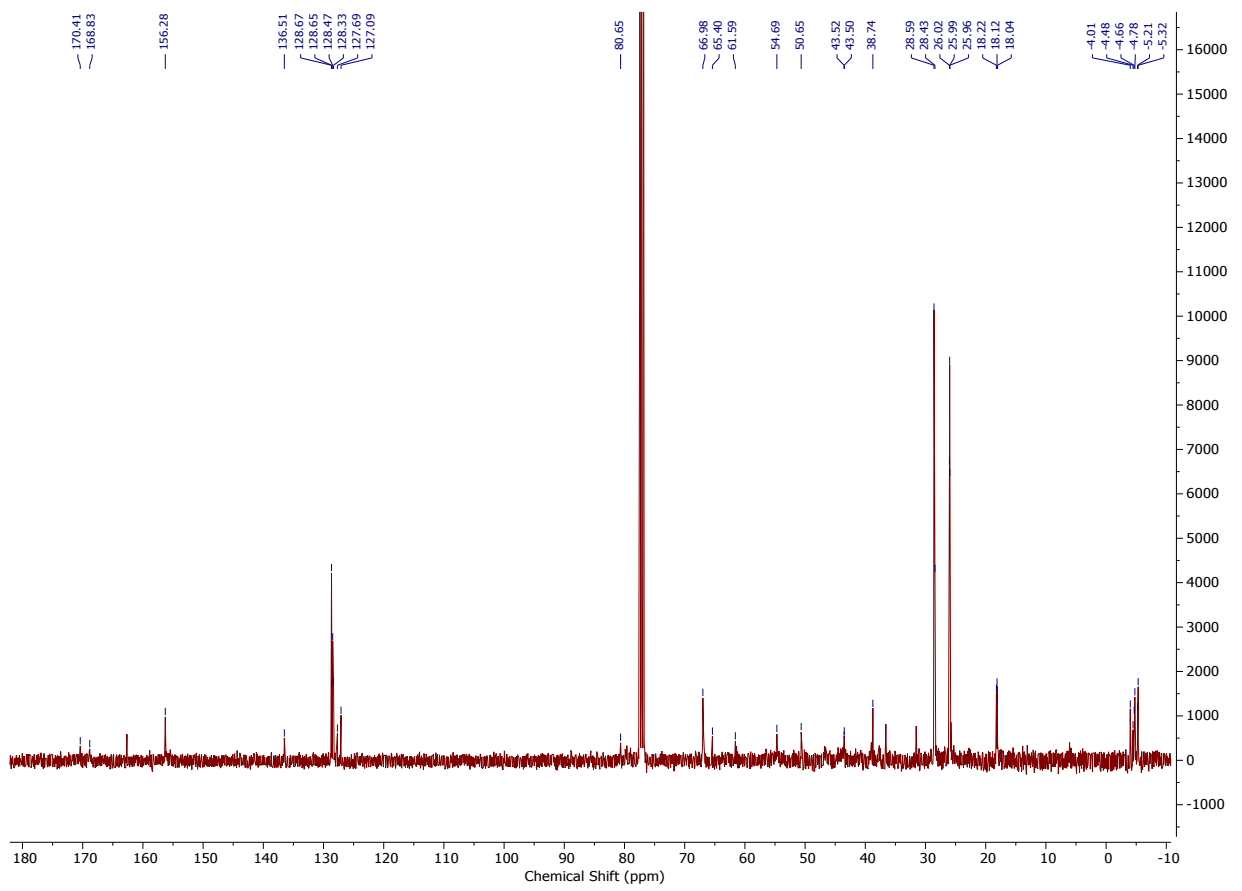




\section{Antimicrobial testing experimental procedures}

The following organisms were tested at the Hans Knöll Institute in Jena, for their susceptibility to the compounds: Bacillus subtilis ATCC6633, Staphylococcus aureus SG511, Staphylococcus aureus 134/94, Escherichia coli (ATCC458), Pseudomonas Aeruginosa (SG137), Sporobolomyces salmonicolor (Basidiomycetes yeast) (SBUG 5549), Aspergillus fumigatus (ATCC46645). The bacteria were propagated in Mueller Hinton Broth (Oxoid). Yeast was propagated in YPD (yeast extract peptone dextrose): $1 \%$ yeast extract, $10 \mathrm{~g} ; 2 \%$ peptone, $20 \mathrm{~g} ; 2 \%$ agar, $20 \mathrm{~g} ; 2 \%$ dextrose (glucose), $20 \mathrm{~g}$; Sigma-Y1375. All bacteria and yeast suspensions were freshly made. The cultures were incubated at $37^{\circ} \mathrm{C}$ for $16 \mathrm{~h}$. Afterwards, the bacteria concentration from each culture was determined, and $34 \mathrm{ml}$ of nutrient agar was loaded with 107 cells. These cultures were stored at $6-8{ }^{\circ} \mathrm{C}$ and could be used for seven days for the preparation of test plates. $34 \mathrm{ml}$ of nutrient agar was liquefied and inoculated with the calculated amount of inoculum bacteria suspension at $48-50{ }^{\circ} \mathrm{C}$, a temperature suitable for maintaining the viability of microorganisms. The inoculated nutrient media $(34 \mathrm{ml}$ each) were immediately poured into the prepared test plates ( $3 \mathrm{~mm}$ layer). With a punching device, 12 holes per test plate were punched out. For inhibiting bacterial growth, ciprofloxacin was used as a reference substance. The test substances were dissolved in distilled water, and $5 \mu \mathrm{g} \mathrm{ml}^{-1}$ and $50 \mu \mathrm{l}$ was added to each bacterial culture. For inhibition of fungal growth, amphotericin B served as the reference substance.

Table S2. Agar disk diffusion screening of PamB2.

\begin{tabular}{lcc} 
Microorganism & PaenB2_3a & Ciprofloxacin \\
\cline { 2 - 3 } & \multicolumn{2}{c}{ Zone of inhibition in mm } \\
\hline Gram-positive: & \multicolumn{2}{c}{20} \\
\hline Bacillus subtillis (ATCC6633) & 18 & 19 \\
Staphylococcus Aureus (SG511) & 13 & 25 \\
\hline Gram-negative: & 13 & 25 \\
\hline Escherichia coli (ATCC458) & & \\
Pseudomonas Aeruginosa (SG137) & & \\
&
\end{tabular}




\section{In vitro activation assay of PamZ}

A reaction mixture consisted of $0.5 \mathrm{mM}$ PamB2_3, $7.5 \mu \mathrm{M}$ PamZ, $1 \mathrm{mM}$ acetyl-CoA, $1.5 \mathrm{mM}$ sodium phosphate buffer $(\mathrm{pH} 7.8)$. Also, samples were prepared each without enzyme and cosubstrate as negative controls. The reaction mixture was incubated at $30^{\circ} \mathrm{C}$ for $2 \mathrm{~h}$. PamZ was removed by Amicon centrifugal filters (Merck KGaA, Germany) using a $10 \mathrm{kDa}$ molecular weight cut-off filter. After removal of the protein, the reaction mixture was tested against $B$. megaterium as indicator strain by agar diffusion assay and analyzed with HPLC-ESI-MS.

\section{Mass spectrometry analysis for in vitro activation assay}

A LTQ-Orbitrap XL hybrid ion trap-orbitrap (Thermo Fisher Scientific GmbH, Bremen, Germany) was used to verify the in vitro activation assays. The LTQ-Orbitrap XL was attached to an analytical HPLC 1200 Infinity system (Agilent Technologies, Waldbronn, Germany) and equipped with a HPLC column (Poroshell 120, EC-C18, $2.7 \mu \mathrm{m}, 2.1 \times 50 \mathrm{~mm}$, Agilent Technologies, Waldbronn, Germany). HPLC was run with a linear gradient using water plus $0.1 \%(\mathrm{v} / \mathrm{v})$ formic acid as solvent $A$ and acetonitrile plus $0.1 \%(\mathrm{v} / \mathrm{v})$ formic acid as solvent $B$ from $5 \%(\mathrm{v} / \mathrm{v})$ to $100 \%(\mathrm{v} / \mathrm{v})$ solvent $B$ for $6 \mathrm{~min}$, followed by an isocratic gradient of $100 \%(\mathrm{v} / \mathrm{v})$ solvent $B$ for $2 \mathrm{~min}$. The column was equilibrated with $5 \%(\mathrm{v} / \mathrm{v})$ solvent $B$ for $2 \mathrm{~min}$. The flow rate was set to $0.5 \mathrm{~mL} / \mathrm{min}$. 


\section{In vitro translation inhibition assays}
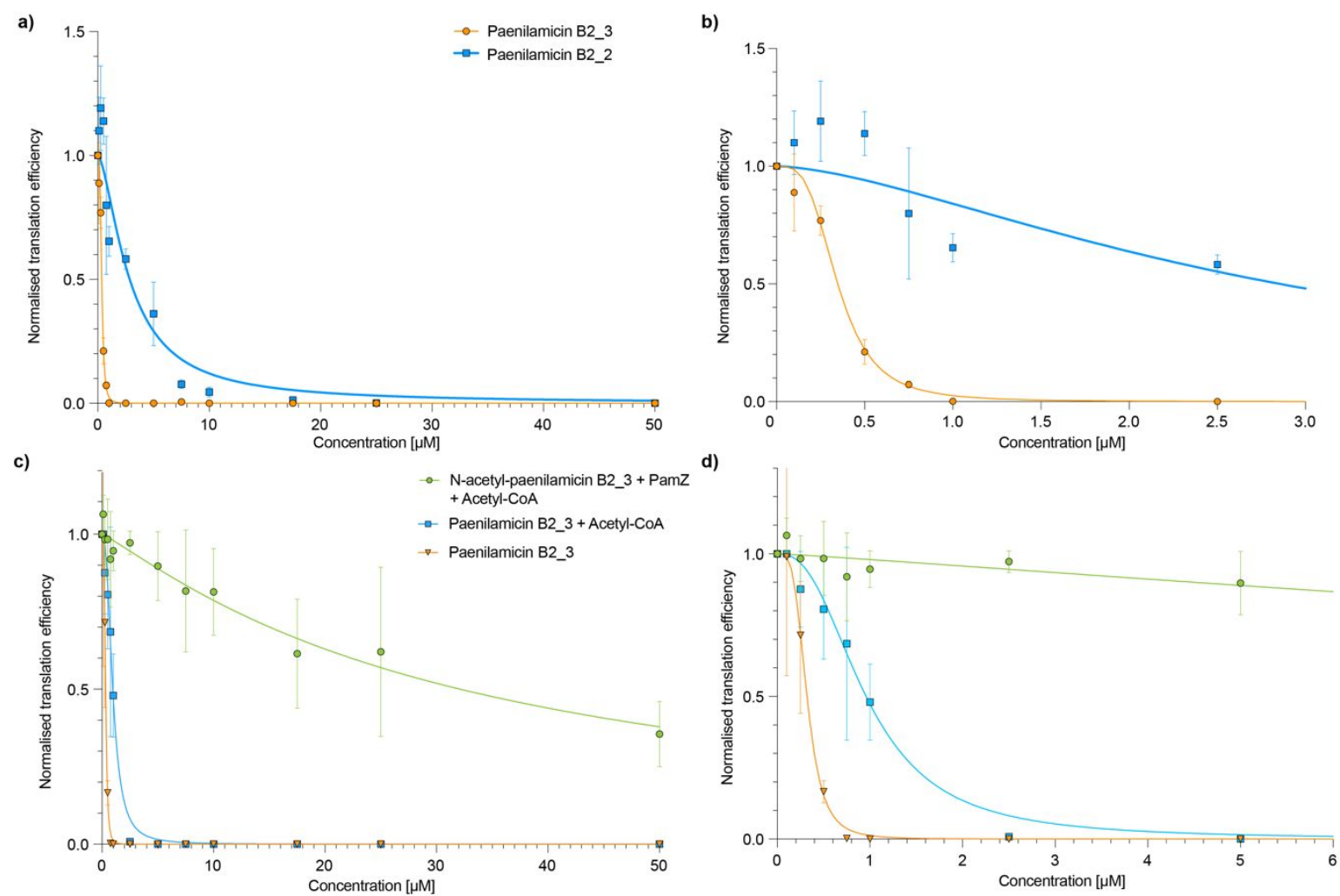

Figure S5. In vitro translation inhibition activities. Normalized in vitro translation firefly luciferase assay (a) and a zoomed in view of the concentration range from 0-3 $\mu \mathrm{M}(\mathbf{b})$. Translation activity of $E$. coli PURExpress ${ }^{\circledR}$ system $^{9}$ in absence $(0 \mu \mathrm{M})$ and presence of increasing concentrations $(0.1-50 \mu \mathrm{M})$ of PamB2_2 (a,b, blue) and PamB2_3 (a, b, orange). The luminescence was normalized to $100 \%$ for the reaction in absence of the antibiotic. The error bars represent the standard deviation of the mean from three biological replicates, with individual data points shown as circles. The $\mathrm{IC}_{50}$ of PamB2_2 $(2.86 \mu \mathrm{M})$ and PamB2_3 $(0.38 \mu \mathrm{M})$ were estimated using linear regression and taken as the concentration where the peptides inhibited translation by $50 \%$ compared to the normalized control. Normalized in vitro translation firefly luciferase assay (c) with a zoom in of the concentration range from 0-6 $\mu \mathrm{M}$ (d). Translation activity of E. coli PURExpress ${ }^{\circledR}$ system $^{9}$ in absence $(0 \mu \mathrm{M})$ and presence of PamB2_3 (c, d orange), PamB2_3 in addition of acetyl-CoA and phosphate buffer (c, d, blue) and N-acetyl-PamB2_3 (c, d, green) with increasing concentrations $(0.1-50 \mu \mathrm{M})$. The absolute luminescence was normalized to $100 \%$ for the reaction in absence of the antibiotic. For the samples containing acetyl-CoA the absolute luminescence was normalized to $0.1 \mu \mathrm{M}$ of antibiotic to account for the impact of acetyl-CoA on the translation system. The error bars represent the standard deviation of the mean from three biological replicates. The curves were calculated using non-linear regression. The $\mathrm{IC}_{50}$ of $\mathrm{N}$-acetyl-PamB2_3 is approximately $31.9 \mu \mathrm{M}$.

\section{Comment to the data in Figure S5}

Lower concentrations of the incorrect diastereomer PamB2_2 showed to have a stimulating effect on the firefly luciferase in vitro assay (Figure S5, a, b, blue). It was not possible to determine the reason of the observed effect. However, the inhibitory potential of PamB2_2 was shown for concentrations higher than $1 \mu \mathrm{M}$ with lower standard deviation and seem not to be interfered by the stimulating effect seen 
for lower concentrations. The non-linear regression was calculated in Graphpad Prism and the $\mathrm{IC}_{50}$ value of $2.86 \mu \mathrm{M}$ of the incorrect diastereomer PamB2_2 is only a rough estimate.

Different from the PamB2_2 the non-linear regression of the correct diastereomer PamB2_3 and the corresponding $\mathrm{IC}_{50}$ value of $0.38 \mu \mathrm{M}$ was estimated with high precision, as seen from the standard deviation of the biological replicates. The PamB2_3 shows a seven times higher inhibitory effect on translation. Despite the larger standard deviation for the $\mathrm{N}$-acetyl-PamB2_3 (Figure S5, c, d, green) a hundred times decrease of translation inhibition resulting from the $\mathrm{N}$-acetylation of the PamB2_3 with an estimated $\mathrm{IC}_{50}$ of $31.9 \mu \mathrm{M}$ was shown when compared to the $\mathrm{IC}_{50}$ of $0.38 \mu \mathrm{M}$ of PamB2_3 (Figure S5, c, d, orange).

In order to address the addition of acetyl-CoA to the firefly luciferase assay as a substrate for PamZ enzymatic reaction, a control reaction with increasing antibiotic concentration and a proportion of 1:100 acetyl-CoA to PamB2_3 was tested. CoA is known to affect firefly luciferase luminescence as it acts as a substrate, while acetyl-CoA is not reported to interfere with this assay. ${ }^{10}$ However, the addition of low amounts of acetyl-CoA with the antibiotic shows an increase in the absolute luminescence values and shifts the $\mathrm{IC}_{50}$ from $0.38 \mu \mathrm{M}$ to $1 \mu \mathrm{M}$ for PamB2_3. For this reason, the absolute luminescence was normalized to the reaction containing the lowest amount of antibiotic or acetyl-CoA, respectively. While the $\mathrm{IC}_{50}$ of the PamB2_3 was affected by the addition, the stimulating effect on the luciferase assay was not able to suppress the activity of the antibiotic PamB2_3. This supports that the actual acetylation of the PamB2_3 is the reason for the reduction in the inhibitory capabilities of the $\mathrm{N}$-acetyl-Pam B2_3.

\section{In vitro translation assay method}

The in vitro translation assay was carried out as described previously ${ }^{11,12}$ using the $E$. coli PURExpress ${ }^{\circledR}$ system (NEB E6800S). $1 \mu \mathrm{L}$ of antibiotic solution was added to $5 \mu \mathrm{L}$ of PURExpress ${ }^{\circledR}$ reaction mix. Each reaction contained $1 \mu \mathrm{g}$ of mRNA encoding the firefly luciferase, which was in vitro transcribed from a pIVEX-2.3MCS vector containing the firefly luciferase gene using T7 polymerase (Thermo Scientific ${ }^{\mathrm{TM}}$ ). The reaction mix was incubated for $30 \mathrm{~min}$ at $32^{\circ} \mathrm{C}$ while shaking. Reactions were stopped with $1 \mu \mathrm{L} \mathrm{Kanamycin}(50 \mathrm{mg} / \mathrm{mL}$ ) and transferred into a 96-well microplate (Greiner Lumitrac, nonbinding, white, chimney). $40 \mu \mathrm{L}$ of luciferase assay substrate solution (Promega E1501) was added and luminescence was measured using a plate reader (Tecan Infinite ${ }^{\circledR} 200$ Pro). Nuclease free water was added instead of antibiotic and/or mRNA as negative control. Absolute luminescence values were normalized using reactions without antibiotic or normalized to $0.1 \mu \mathrm{M}$ antibiotic in presence of acetylCoA. All assays were done as triplicates with individually prepared reaction mix.

\section{Data analysis and Figure preparation of in vitro translation assay}

Data was normalized and analysed using GraphPad Prism V9.1.2. $I C_{50}$ s were calculated in Prism by using non-linear regression and visualized as curves. Figures were created in Adobe Illustrator CC V24.3 (www.adobe.com/products/illustrator.html). 


\section{In vivo function experimental procedures}

a)

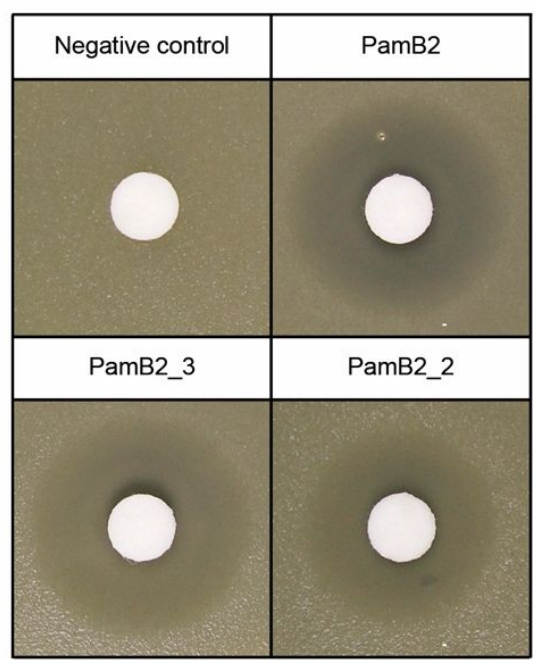

b)

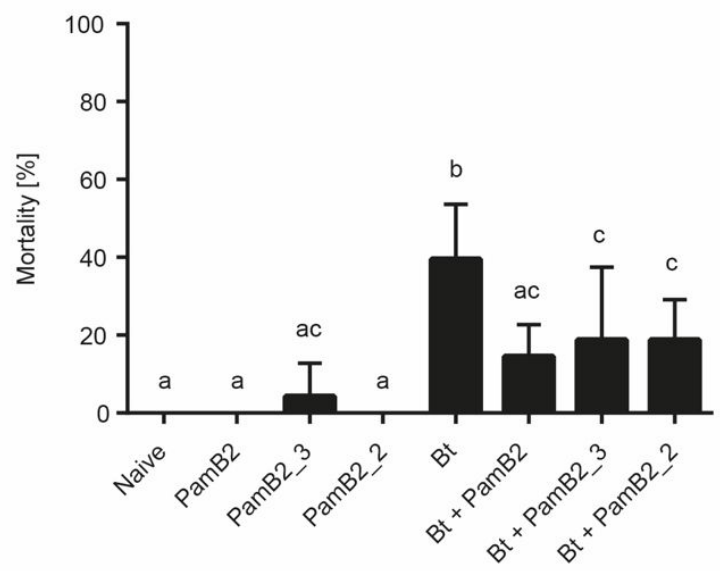

Figure S6. Activity of natural PamB2, synthesized PamB2_3 and synthesized PamB2_2 against B. thuringiensis (Bt). a) Agar diffusion assay showing the antibacterial activity of PamB2, PamB2_3, and PamB2_2 (30 $\mu$ g each) on Bt. b) Honey bee larvae feeding assay. Different letters above the error bars (mean $\pm \mathrm{SD}$ ) indicate significant differences $(p<0.05)$ among groups (ordinary one-way ANOVA with Fisher's LSD test).

\section{Bacterial strain and culture conditions}

The commercially available product B401® (Vita (Europe) Ltd., Basingstoke, UK) contains spores and protein crystals from the entomopathogenic bacterium Bacillus thuringiensis Berliner var. Aizawa and is used in apiculture to prevent damage caused by wax moth larvae (Galleria mellonella). Hence, honey bee larvae are regularly exposed to these $B$. thuringiensis spores and any interaction with Pam would be of concern. $B 401 \circledR$ contains $50.45 \times 10^{8} \mathrm{cfu} / \mathrm{ml}$ B. thuringiensis spores as determined by plating out serial dilutions on Mueller-Hinton-yeast-phosphate-glucose-pyruvate (MYPGP) ${ }^{13}$ agar plates, followed by incubation at $37^{\circ} \mathrm{C}$, overnight. The determination of the cfu/ml via plating out $5 \times 200 \mu$ of each dilution was performed three times.

\section{Agar diffusion assay}


The activity of natural PamB2, synthetic PamB2_3, and synthetic PamB2_2 was examined in an agar diffusion assay. A pre-culture with MYPGP medium was inoculated with a loop of $B$. thuringiensis bacterial material and incubated gently shaking at $37^{\circ} \mathrm{C}$ overnight. Agar plates were poured from liquid lukewarm MYPGP agar which was previously inoculated with the pre-culture to obtain an optical density at $600 \mathrm{~nm}\left(\mathrm{OD}_{600}\right)$ of 0.05 . Filter disks were soaked with $20 \mu \mathrm{l}$ of the different paenilamicin dissolved in MilliQ water (30 $\mu$ g per filter disk). Filter disks with $20 \mu l$ MilliQ water served as negative control. Filter disks were dried at room temperature and allocated to the agar plates with sterile forceps. Incubation of the plates took place at $37^{\circ} \mathrm{C}$ overnight. Zones of inhibition were indicative for paenilamicin activity against $B$. thuringiensis.

\section{Honey bee (Apis mellifera) larval feeding assay}

To analyze a putative antibacterial effect of PamB2 on $B$. thuringiensis in bee larvae ( $A$. mellifera), a larval feeding assay with natural PamB2 purified from $P$. larvae supernatant, synthesized PamB2_2, and synthesized PamB2_3 together with B. thuringiensis spores (B401®) was performed. Honey bee larvae were reared for 7 days as previously described ${ }^{14,15}$. Newly hatched honey bee larvae were grafted into 24-well plates which contained larval food $(3 \%(\mathrm{w} / \mathrm{v})$ fructose, $3 \%(\mathrm{w} / \mathrm{v})$ glucose, and $66 \%$ $(\mathrm{v} / \mathrm{v})$ royal jelly) within the first $24 \mathrm{~h}$ post-hatching (day 0 ). All honey bee larvae received normal food until day three post-hatching. On day three post-hatching, the honey bee larvae were allocated randomly into the treatment groups, either receiving normal larval food (naivecontrol), larval food supplemented with either one of the different PamB2 (final concentration of $15 \mu \mathrm{g} / \mathrm{ml}$ ), larval food supplemented with $B$. thuringiensis spores (final concentration $6 * 10^{6} \mathrm{cfu} / \mathrm{ml}$ ), or larval food supplemented with either one of the different PamB2 (final concentration of $15 \mu \mathrm{g} / \mathrm{ml}$ ) together with $B$. thuringiensis spores (final concentration of $6 * 10^{6} \mathrm{cfu} / \mathrm{ml}$ ) until the larvae stopped feeding and started to defecate which labels the onset of pupation (about day 7 post-hatching). The honey bee larvae were constantly kept at $35^{\circ} \mathrm{C}$ and checked for mortality every day for 4 days. The experiment was repeated four times with 12 larvae per group each. Data were analyzed with ordinary one-way ANOVA with Fisher's LSD test with GraphPad Prism 6 (La Jolla, CA, USA). 


\section{References}

(1) Müller, S.; Garcia-Gonzalez, E.; Mainz, A.; Hertlein, G.; Heid, N. C.; Mösker, E.; Van Den Elst, H.; Overkleeft, H. S.; Genersch, E.; Süssmuth, R. D. Paenilamicin: Structure and Biosynthesis of a Hybrid Nonribosomal Peptide/Polyketide Antibiotic from the Bee Pathogen Paenibacillus Larvae. Angew. Chemie - Int. Ed. 2014, 53 (40), 10821-10825. DOI: 10.1002/anie.201404572.

(2) Lee, W.; Tonelli, M.; Markley, J. L. NMRFAM-SPARKY: Enhanced Software for Biomolecular NMR Spectroscopy. Bioinformatics 2015, 31 (8), 1325-1327. DOI:

10.1093/bioinformatics/btu830.

(3) Lee, W.; Cornilescu, G.; Dashti, H.; Eghbalnia, H. R.; Tonelli, M.; Westler, W. M.; Butcher, S. E.; Henzler-Wildman, K. A.; Markley, J. L. Integrative NMR for Biomolecular Research. J. Biomol. NMR 2016, 64 (4), 307-332. DOI: 10.1007/s10858-016-0029-x.

(4) Wishart, D. S.; Bigam, C. G.; Yao, J.; Abildgaard, F.; Dyson, H. J.; Oldfield, E.; Markley, J. L.; Sykes, B. D. 1H, 13C and 15N Chemical Shift Referencing in Biomolecular NMR. J. Biomol. NMR 1995, 6 (2), 135-140. DOI: 10.1007/BF00211777.

(5) Markley, J. L.; Bax, A.; Arata, Y.; Hilbers, C. W.; Kaptein, R.; Sykes, B. D.; Wright, P. E.; Wüthrich, K. Recommendations for the Presentation of NMR Structures of Proteins and Nucleic Acids. J. Mol. Biol. 1998, 280 (5), 1-23. DOI: 10.1006/jmbi.1998.1852.

(6) Williamson, M. P. Using Chemical Shift Perturbation to Characterise Ligand Binding. Prog. Nucl. Magn. Reson. Spectrosc. 2013, 73, 1-16. DOI: 10.1016/j.pnmrs.2013.02.001.

(7) Koźmiński, W.; Nanz, D. Sensitivity Improvement and New Acquisition Scheme of Heteronuclear Active-Coupling-Pattern-Tilting Spectroscopy. J. Magn. Reson. 2000, 142 (2), 294-299. DOI: 10.1006/jmre.1999.1939.

(8) Hwang, T. L.; Shaka, A. J. Cross Relaxation without TOCSY: Transverse Rotating-Frame Overhauser Effect Spectroscopy. J. Am. Chem. Soc. 1992, 114 (8), 3157-3159. DOI: 10.1021/ja00034a083.

(9) Shimizu, Y.; Inoue, A.; Tomari, Y.; Suzuki, T.; Yokogawa, T.; Nishikawa, K.; Ueda, T. Cell-Free Translation Reconstituted with Purified Components. Nat. Biotechnol. 2001, 19 (8), 751-755. DOI: $10.1038 / 90802$.

(10) Fraga, H.; Fernandes, D.; Fontes, R.; Esteves Da Silva, J. C. G. Coenzyme A Affects Firefly Luciferase Luminescence Because It Acts as a Substrate and Not as an Allosteric Effector. FEBS J. 2005, 272 (20), 5206-5216. DOI: 10.1111/j.1742-4658.2005.04895.x.

(11) Mardirossian, M.; Pérébaskine, N.; Benincasa, M.; Gambato, S.; Hofmann, S.; Huter, P.; Müller, C.; Hilpert, K.; Innis, C. A.; Tossi, A.; Wilson, D. N. The Dolphin Proline-Rich Antimicrobial Peptide Tur1A Inhibits Protein Synthesis by Targeting the Bacterial Ribosome. Cell Chem. Biol. 2018, 25 (5), 530-539. DOI: 10.1016/j.chembiol.2018.02.004.

(12) Seefeldt, A. C.; Nguyen, F.; Antunes, S.; Pérébaskine, N.; Graf, M.; Arenz, S.; Inampudi, K. K.; Douat, C.; Guichard, G.; Wilson, D. N.; Innis, C. A. The Proline-Rich Antimicrobial Peptide Onc112 Inhibits Translation by Blocking and Destabilizing the Initiation Complex. Nat. Struct. Mol. Biol. 2015, 22 (6), 470-475. DOI: 10.1038/nsmb.3034. 
(13) Dingman, D. W.; Stahly, D. P. Medium Promoting Sporulation of Bacillus Larvae and Metabolism of Medium Components. Appl. Environ. Microbiol. 1983, 46 (4), 860-869. DOI: 10.1128/aem.46.4.860-869.1983.

(14) Genersch, E.; Ashiralieva, A.; Fries, I. Strain- and Genotype-Specific Differences in Virulence of Paenibacillus Larvae Subsp. Larvae, a Bacterial Pathogen Causing American Foulbrood Disease in Honeybees. Appl. Environ. Microbiol. 2005, 71 (11), 7551-7555. DOI: 10.1128/AEM.71.11.7551-7555.2005.

(15) Genersch, E.; Forsgren, E.; Pentikäinen, J.; Ashiralieva, A.; Rauch, S.; Kilwinski, J.; Fries, I. Reclassification of Paenibacillus Larvae Subsp. Pulvifaciens and Paenibacillus Larvae Subsp. Larvae as Paenibacillus Larvae without Subspecies Differentiation. Int. J. Syst. Evol. Microbiol. 2006, 56 (3), 501-511. DOI: 10.1099/ijs.0.63928-0. 